\title{
MOLECULAR MECHANISMS MODULATING CHONDROGENESIS
}

Catalina Galeano Garcés

2019 
This dissertation has been approved by:

\section{Supervisor:}

Prof. Dr. H.B.J Karperien (University of Twente)

\section{Co-supervisor:}

Prof. Dr. A. van Wijnen (Mayo Clinic)

Cover design: Daniel Escobar-Naranjo

Printed by: IPSKAMP printing

ISBN: 978-90-365-4814-4

DOI: $10.3990 / 1.9789036548144$

URL: https://doi.org/10.3990/1.9789036548144

(C) Catalina Galeano Garcés, 2019, Enschede, the Netherlands. All rights reserved. No parts of this thesis may be reproduced, stored in a retrieval system or transmitted in any form or by any means without permission of the author. Alle rechten voorbehouden. Niets uit deze uitgave mag worden vermenigvuldigd, in enige vorm of op enige wijze, zonder voorafgaande schriftelijke toestemming van de auteur. 


\title{
MOLECULAR MECHANISMS MODULATING CHONDROGENESIS
}

\author{
DISSERTATION \\ to obtain \\ the degree of doctor at the University of Twente, \\ on the authority of the rector magnificus, \\ Prof. Dr. T.T.M. Palstra, \\ on account of the decision of the Doctorate Board, \\ to be publicly defended \\ on Wednesday, July $17^{\text {th }} 2019$ at 14.45 hours
}

by

Catalina Galeano Garcés

Born on June $11^{\text {th }} 1990$

in Medellin, Colombia 


\section{Members of the Graduation Committee}

\section{Chairman/secretary:}

Prof. dr. J.L. Herek

\section{Supervisors:}

Prof. dr. H.B.J. Karperien (University of Twente)

Prof. dr. A. van Wijnen (Mayo Clinic)

\section{Members:}

Prof. dr. ir. P. Jonkheijm (University of Twente)

Prof. dr. G. Storm (University of Twente)

Prof. dr. G.J.V.M van Osch (Erasmus Medical Center Rotterdam)

Prof. dr. M.A. Tryfonidou (Utrecht University)

Dr. T. Welting (Maastricht University Medical Center)

Dr. J. N. Post (University of Twente) 


\section{Summary}

In the last decade various tissue engineering strategies have emerged for articular cartilage repair. MSCs are currently being used in various clinical trials to exploit the multilineage capacity and differentiation potential of these cells. Altough promising clinical results have been seen, controlling the commitment and differentiation of the cells to the expected pathway remains challenging. Our area of investigation will focus its efforts in achieving the understanding of proper molecular mechanisms that could control the commitment and differentiation of the cells whilst avoiding a hypertrophic or fibrous phenotype. The first and second chapters will provide a background, significance, and an overview on the use of environmental conditions, such as hypoxia, for cartilage repair. Chapter three focuses on validating chondrogenesis of adipose derived stem cells (aMSCs) in low oxygen cultures. Cell type specific effects of low oxygen and 3D environments indicated that genetic programming of aMSCs to a chondrocytic phenotype is effective under hypoxic conditions, as evidenced by increased expression of cartilage-related biomarkers and biosynthesis of a glycosaminoglycan positive matrix. Chapter four and five draw major attention to the molecular mechanisms by which miRNAs could direct chondrogenesis during the hypoxic response of MSCs and explores the potential of microRNA-210 (miR210) to enhance in vitro chondrogenic differentiation of stem cells. Hypoxic regulated miR-210 was found to be essential in the regulation of genes in charge of several functions crucial for cartilage development, chondrogenic differentiation and the oxidative stress response. Exogenous miR-210 expression can potentially be utilized instead of inducing chondrogenic differentiation using TGFß1 in a three dimensional culture under low oxygen, to promote chondrogenesis of MSCs while inhibiting their hypertrophic differentiation.

Chondrogenesis improvement was evidenced by increased expression of cartilaginous markers, proteoglycan deposition and collagen II protein content. 
Chapter six will reveal the effects of synovial fluid on in vitro models of primary chondrocytes and mesenchymal stem cells. Metabolic activity assays on primary chondrocytes and aMSCs showed both cell types survived and proliferated during culture with synovial fluid (SF). Moreover, synovial fluid seems to be permissive for chondrogenic differentiation of aMSCs in the presence of chondrogenic cocktail, which was confirmed by positive type II collagen immunohistochemistry. Our results serve as an initial screening of the possibilities of SF to replace fetal bovine serum as a culture supplement for in vitro expansion of human primary chondrocytes and aMSCs. Chapter seven explores the role of ZNF648, a cartilage specific transcription factor, expressed in immature cartilage and growth plate, to understand its role during cartilage development and to provide a molecular mechanism to create a competent tissue for cartilage repair. In the growth plate, this ZNF648 protein seems to maintain a chondrocytic phenotype of immature cells, whereas in later stages of cartilage maturation its expression is reduced. An enhanced expression of zinc finger 648 (ZNF648) increased collagen type II (COL2A1) expression in growth plate derived chondrocytes while reducing its expression in articular chondrocytes, which suggested an important regulatory mechanism during early development of the chondrocytes in the growth plate but not in terminally differentiated chondrocytes such as the ones in articular cartilage. Thus, development of novel approaches using ZNF648 to improve and maintain cartilage homeostasis is thought to play an essential role in future clinical therapies. Ultimately, chapter eight will provide a general discussion of the results presented in this thesis and presents future perspectives for the use of stem cells in cartilage regeneration therapies. 


\section{Table of Contents}

1. GENERAL INTRODUCTION AND THESIS OUTLINE ............................. 13

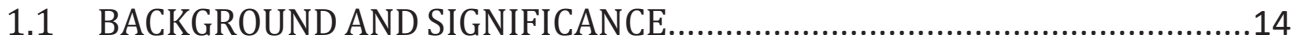

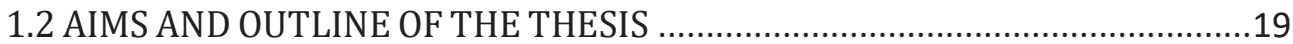

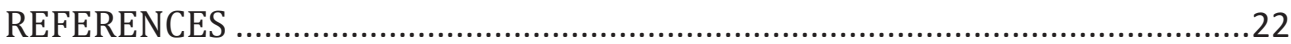

2. HYPOXIA REGULATORY MECHANISMS DURING CARTILAGE

DEVELOPMENT AND CARTILAGE-TISSUE ENGINEERING. ............................ 26

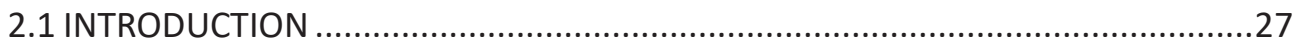

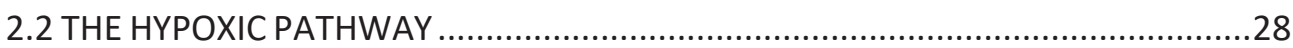

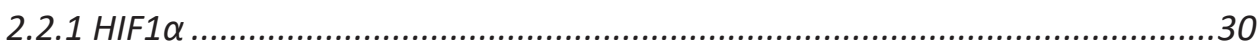

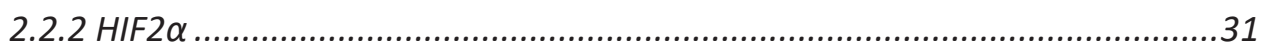

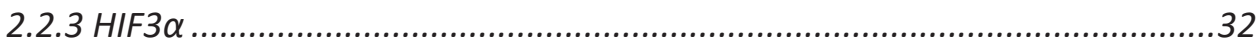

2.3 HYPOXIA DURING SKELETAL FORMATION IN MAMMALS................................32

2.4 CROSSTALK BETWEEN HYPOXIA AND CARTILAGE RELATED PATHWAYS .............34

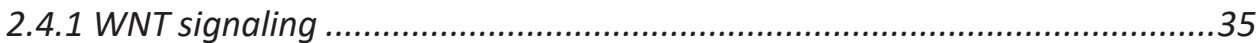

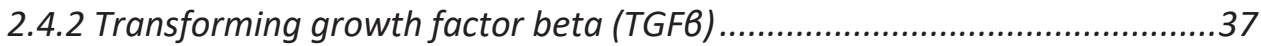

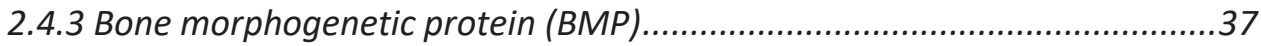

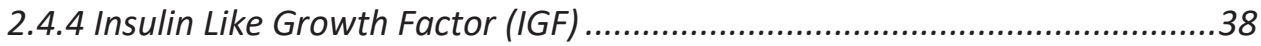

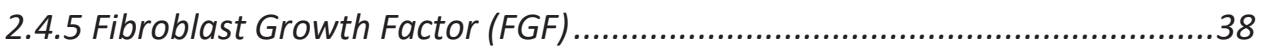

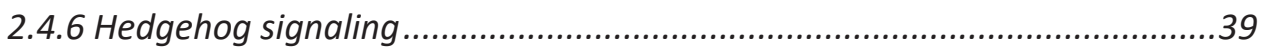

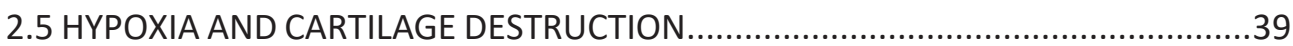

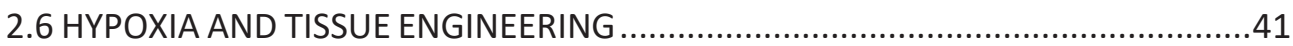

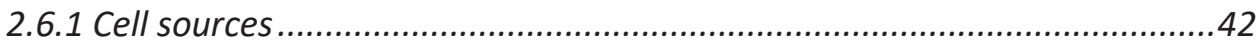

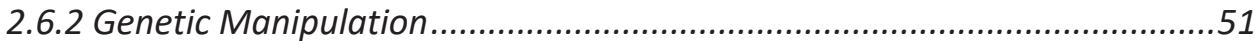

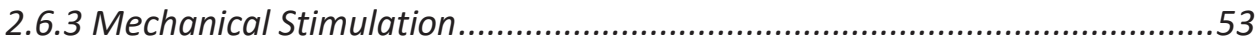

2.6.4 Scaffold free cultures and biomaterials for cartilage tissue engineering....55

2.7 CHALLENGES, PROSPECTIVES AND FUTURE DIRECTIONS ................................57

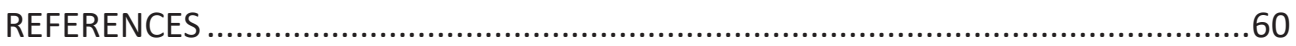

3. HYPOXIA PROMOTES IN VITRO CHONDROGENIC DIFFERENTIATION OF ADIPOSE DERIVED STEM CELLS (AMSCS) IN THREE-DIMENSIONAL

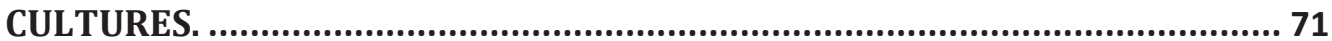

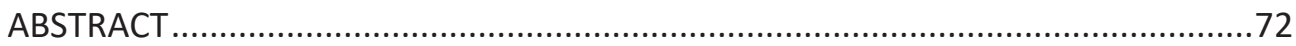

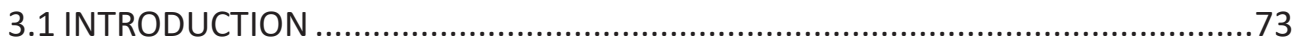




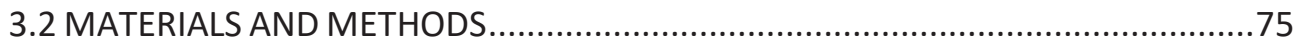

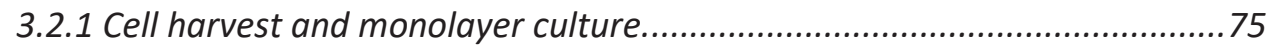

3.2.2 Fabrication of nanofibrous poly-e-caprolactone (PCL) scaffolds.................76

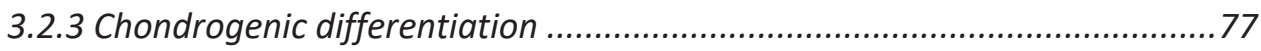

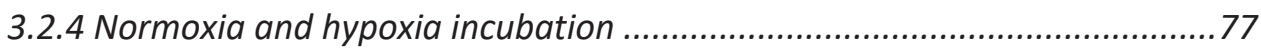

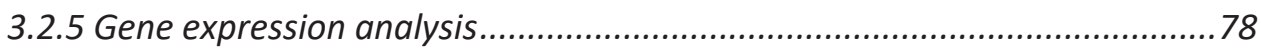

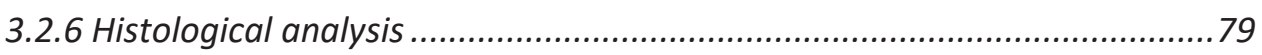

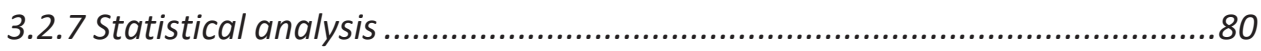

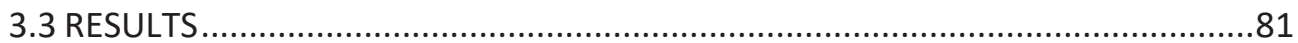

3.3.1 aMSCs undergo chondrogenic differentiation and adopt a phenotype resembling growth plate cartilage .................................................................81

3.3.2 Culture on PCL-scaffolds supports cartilage-specific extracellular matrix formation by both aMSCs and chondrocytes.

3.3.3 Hypoxia promotes cartilage-specific gene expression in aMSCs in both

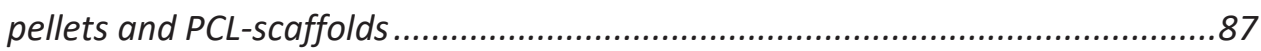

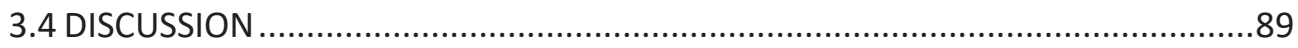

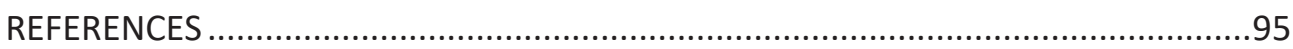

4. INTEGRATED ANALYSIS OF MIRNA-SEQ AND MRNA-SEQ ON HYPOXIC MESENCHYMAL STEM CELLS REVEALS THE ROLE OF HYPOXIA-REGULATED MIRNAS FOR PROMOTING CHONDROGENESIS OF MSCS. ............................ 98

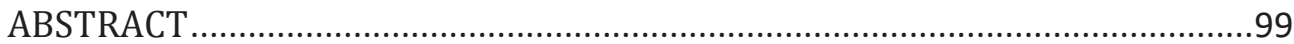

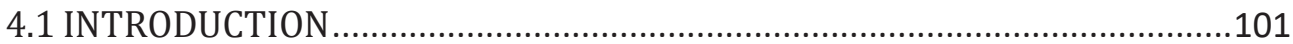

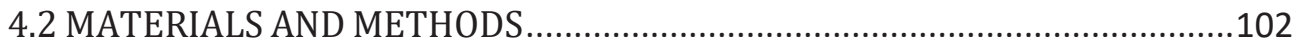

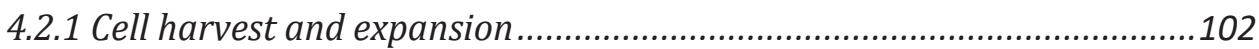

4.2.2 Cell culture under Normoxia and Hypoxia conditions ............................103

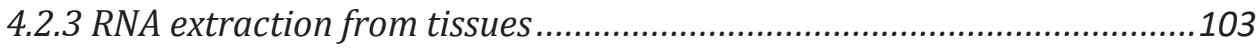

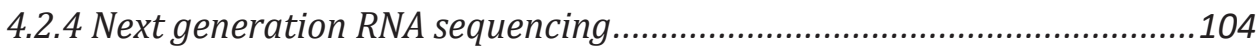

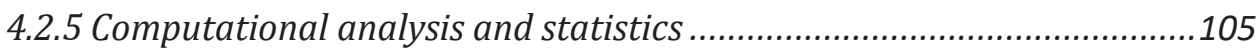

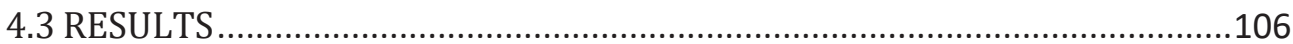

4.3.1 microRNAseq analysis reveals a unique hypoxia induced miR-210 and a clear miRNA footprint of bMSCs on different oxygen conditions.....................106

4.3.2 Modestly induced miRNAs during low oxygen culture do not participate

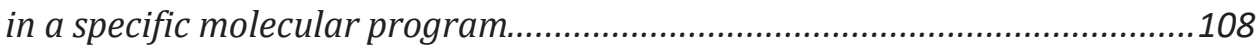
4.3.3 Hypoxia regulated miR-210 gene targets are mostly involved in stress response but also in cartilage regulatory programs......................................110 
4.3.4 Common gene targets of hypoxia inducible factor HIF1 and miRNA-210 in bMSCs play essential roles in tissue development, oxidative stress response and cell cycle regulation.

4.3.5 Targets genes of hypoxia inducible factor HIF1 and microRNA-210 in aMSCs play essential roles in oxidative stress response, tissue homeostasis and inflammation.

4.3.6 MiR-210 may play an important role in the inflammatory response and cartilage homeostasis in healthy cartilage tissues.

4.3.7 Similar regulatory mechanisms enhance chondrogenic differentiation of

MSCs during the hypoxic response. 124

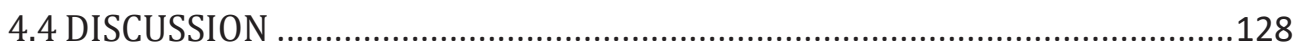

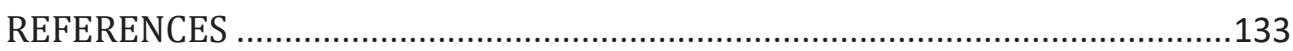

5. MIRNA-210 PROMOTES CHONDROGENESIS OF MSCS ...........................139

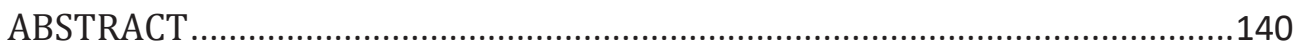

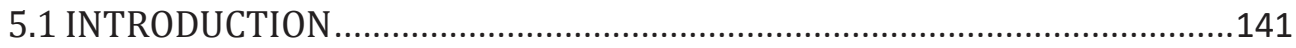

5.2 MATERIALS AND METHODS .............................................................. 142

5.2.1 Monolayer and high-density pellet cultures ...................................... 142

5.2.2 Cell culture under Normoxia and Hypoxia conditions .............................143

5.2 .3 Chondrogenesis of bMSCS. ......................................................... 143

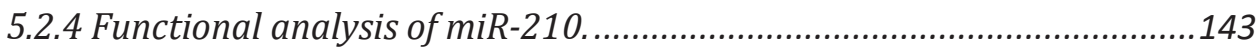

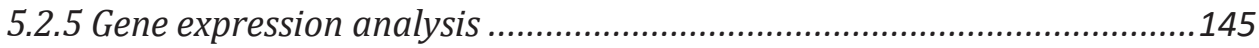

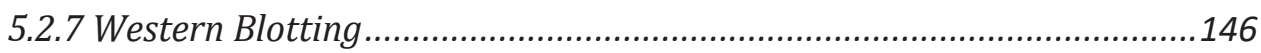

5.2.8 Histological Analysis .................................................................. 147

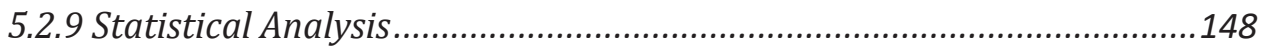

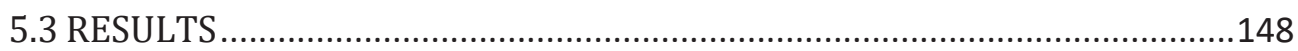

5.3.1 miR-210 is the distinctive hypoxia induced microRNA in MSCs..............148

5.3.2 Hypoxia promotes chondrogenic potential of bMSCs and endogenous

expression of miR-210 ...................................................................... 151

5.3.3 miR-210 overexpression enhances expression of chondrogenic markers in a normoxic environment.

5.3.4 miR-210 inhibition decreases chondrogenic differentiation of MSCs.....154

5.3.5 miR-210 and TGF $\beta 1$ interaction restricts chondrogenic differentiation of bMSCS.

5.3.6 miR-210 promotes chondrogenesis of bMSCs similarly to TGF $\beta 1$ in hypoxic conditions, but interaction of both factors increases hypertrophic differentiation of the cells. 


\section{HUMAN SYNOVIAL FLUID ASSISTS HUMAN PRIMARY CHONDROCYTES} AND MESENCHYMAL STEM CELLS CHONDROGENIC DIFFERENTIATION DURING CULTURE EXPANSION. .168

ABSTRACT. 169

6.1 INTRODUCTION 170

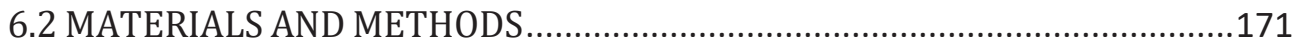

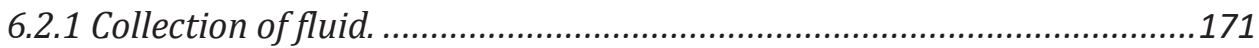

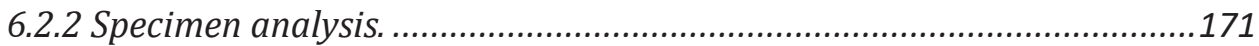

6.2.3 Cell harvest and monolayer culture ....................................................171

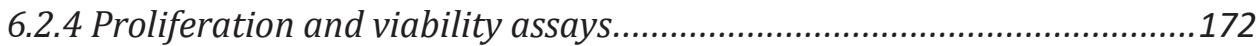

6.2.5 Chondrogenic differentiation potential ...............................................173

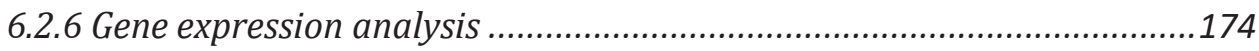

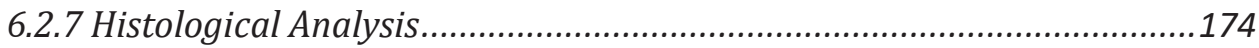

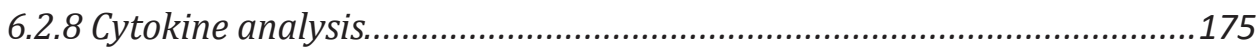

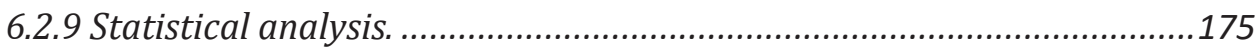

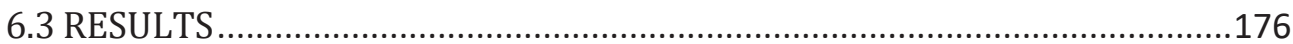

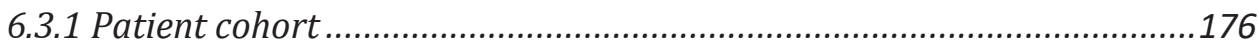

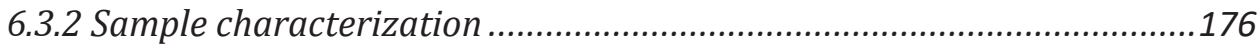

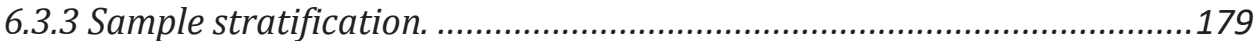

6.3.4 Viability of mesenchymal stem cells and chondrocytes in synovial fluid 183

6.3.5 Glucose importance for human chondrocytes cultured in synovial fluid186

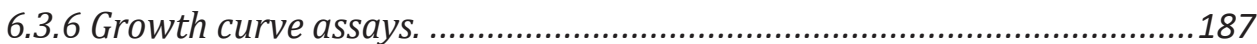

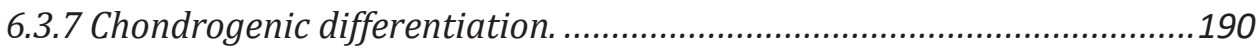

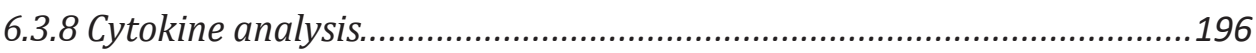

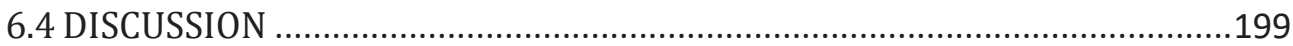

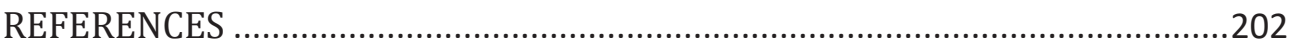

7. NOVEL TRANSCRIPTION FACTOR ZNF648 MIGHT REGULATE CARTILAGE DEVELOPMENT AND HOMEOSTASIS THROUGH THE MODULATION OF CARTILAGE RELATED SIGNALING PATHWAYS. ........................................204

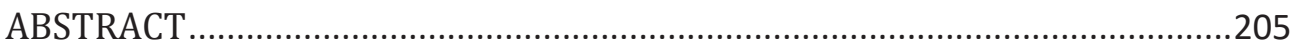

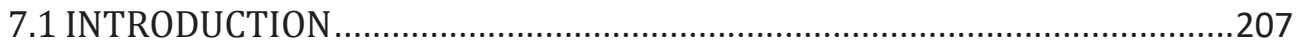

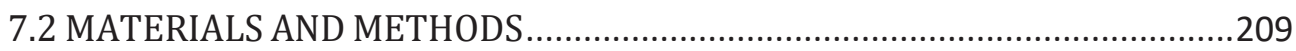

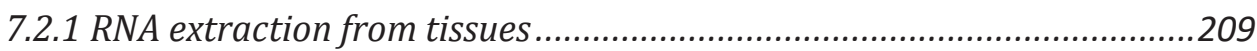


7.2.3 Human cell harvest and expansion

7.2.4 Isolation, culture and analysis of immature mouse chondrocytes (mACs)

7.2.5 Cell culture and expansion of immortalized cells lines... 212

7.2.6 Functional analysis of ZNF648. 212

7.2.7 RNA isolation, reverse transcription and real time quantitative PCR (RTqPCR).

7.2.9 MTS activity assay and Hoechst Staining ...... 214

7.2.10 Histological analysis of human and mouse tissues.

7.2.11 Immunofluorescence

7.2.12 Genetic null mutation of ZNF648 in mouse and evaluation of skeletal phenotyping.... 216

7.2.13 Statistical Analysis. 217

7.3 RESULTS. 218

7.3.1 Identification of ZNF648 as a novel cartilage specific zinc-finger protein

7.3.2 ZNF648 and Zfp648 expression is highly specific to cartilage and not found in bone, following the expression pathways of COL2A1.

7.3.3 Expression of ZNF648 is abundant in primary chondrocytes whereas its expression decreases during cell culture and passaging

7.3.4 ZNF648-mCherry plasmid overexpression in HEK293T cells induced ACAN expression and cell proliferation......

7.3.5 ZNF648 overexpression regulates specific genes in human articular cartilage and growth plate chondrocytes.

7.3.6 Knockdown experiments in human primary chondrocytes imply ZNF648 regulates COL2A1 expression.

7.3.7 ZNF648 controls COL2A1 induction in human immortalized TC28A2 cells and chondrosarcoma cell line L835.

7.3.8 ZNF648 mouse homolog Zfp648 regulates Col2a1 induction in ATDC5 and in immature mouse articular chondrocytes.

7.3.9 Zfp648 null mice survive beyond birth and do not have overt skeletal defects.

7.3.10 Young Zfp648 null mouse exhibit reduced skeletal size and growth plate width with a delayed secondary ossification center and disorganization of growth plate zones. 
7.4 DISCUSSION .234 REFERENCES 239

8. SUMMARY, GENERAL DISCUSSION AND OUTLOOK..............................242

8.1 MeSEnChymal StEm CELLS VERSuS MEDicinal Signaling CELLS .......................243

8.2 HYPOXIA IMPROVES IN VITRO CHONDROGENIC DIFFERENTIATION OF MSCS. ...........244

8.3 SYNOVIAL FLUID IS AN ALTERNATIVE CULTURE SERUM FOR IN VITRO EXPANSION OF MSCS AND CHONDROCYTES FOR CLINICAL THERAPIES

8.4 CARTILAGE SPECIFIC TRANSCRIPTION FACTORS COULD IMPROVE CARTILAGE REGENERATION AND PREVENT OSTEOARTHRITIS DEVELOPMENT................................246

8.5 CONCLUDING REMARKS AND FUTURE PERSPECTIVES. .........................................247

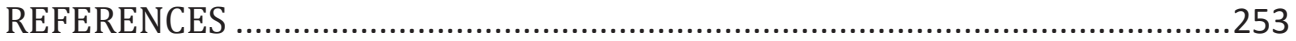

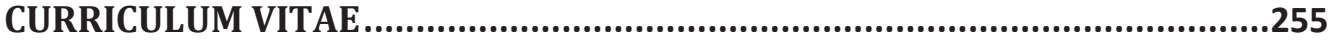

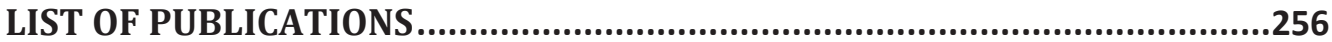

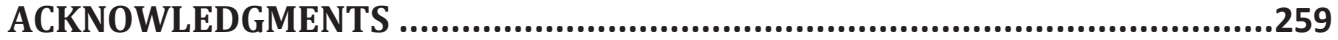




\section{CHAPTER 1}

\section{General introduction and thesis outline}




\subsection{BACKGROUND AND SIGNIFICANCE}

In the last decade various tissue engineering strategies have emerged for articular cartilage repair. MSCs are currently being used in various clinical trials with promising clinical results [1]. Two different notions about MSC therapies have been described. The first notion proposes the term of medicinal signaling cells (MSCs) and relies in the trophic functions of MSCs to support tissue regeneration [2]. Caplan described them as "sentinels" that survey the damage, isolate foreign components, stabilize the injured tissues, provide antibiotics and encysting protection before a medicinal sequence can be initiated to regenerate the damaged tissue [3]. In the second notion, MSCs are describes as stem cells and exploits the multilineage capacity and differentiation potential of these cells. For the development of this thesis, the latter notion, related to the differentiation capacity of these stem cells into chondrocytes, was studied.

Numerous studies have focused on implantation of primary cells or stem cells with a combination of growth factors and biomaterials with the goal of becoming the gold standard for cartilage repair [4]. However, controlling the commitment and differentiation of the cells to the expected pathway remains challenging [5]. The use of stem cells and in particular mesenchymal stem cells (MSCs) from adult sources has emerged as a viable solution to overcome limitations of current cartilage restoration procedures. Multipotent adult MSCs reside in several tissues including bone marrow, skeletal muscle, neural tissue, adipose tissue, and synovium. Currently, most studies for cartilage repair have focused on bone marrow-derived MSCs. As an alternative, human adipose tissue-derived mesenchymal stem cells (aMSCs) are an attractive cellular therapeutic due to the minimally invasive tissue harvest, high abundancy of cells, and rapid expansion ex vivo $[4,6]$. Moreover, these cells are multipotent, can produce musculoskeletal extracellular matrix proteins once confluent, and are able to differentiate into osteogenic and chondrogenic cell lineages [7]. For cartilage tissue homeostasis 
and regeneration, an adequate production of extra cellular matrix (ECM) is necessary to provide the cells with a three dimensional scaffold and to regulate angiogenesis and inflammation processes [8]. MSCs differentiated into chondrocytes are marked by production of sex determining region $\mathrm{Y}$ box 9 (SOX9), aggrecan (ACAN), collagen type II (COL2A1), transforming growth factor$\beta$ (TGF $\beta$ ), fibronectin (FN1), neural cell adhesion molecule $(N-C A M)$ and $\mathrm{N}$ cadherin (CDH2) [1, 9-11]. However, during chondrogenesis of MSCs the expression of cartilage hypertrophy markers, such as collagen type X (COL10A1) and metaloproteinases (MMPs), is also observed [12]. Prevention of hypertrophic differentiation and posterior ossification is important for clinical application of MSCs in cartilage tissue engineering [13].

To maintain the cartilage homeostasis, researchers have used extracellular matrix proteins and combinations of growth factors to promote stem cell attachment, proliferation and initial chondrogenic differentiation [14, 15]. In addition, the role of these factors is to prevent the formation of fibrous cartilage by decreasing collagen type I (COL1A1) expression but maintaining collagen type II (COL2A1) deposition during in vitro chondrogenesiss. For cartilage repair strategies, the most used biomolecules are those who act to initiate signaling molecules cascades during chondrogenesis [15]. TGF $\beta$, BMP and IGF families are the most extensively studied developmental morphogens to stimulate chondrogenic differentiation of stem cells in vitro $[10,16,17]$. In addition, WNT, NOTCH and FGF are interesting candidates based on their in vivo roles during cartilage homeostasis $[18,19]$. Various members of these families have been tested in vitro as chondrogenic inducers in combination with three-dimensional culture environments, mechanical loading and/or genetic approaches. Consequently, stem cells and chondrocytes could closely interact with the biochemical and environmental stimuli during chondrogenesis to maintain a normal chondrocyte phenotype. 
A small number of transcription factors has been described over the years as key factors during cartilage development. These factors play an essential role in regulating standard cartilage markers during chondrogenesis of stem cells, including COL2A1, COL9A1, COL10A1, COL11A1, ACAN and hyaluronan protein link 1 (HAPLN1) [20-24]. Yet the master transcription factor that regulates chondrogenic differentiation is SOX9 [25]. This gene is expressed early during chondrogenesis [26] and its central role is to regulate the expression of main chondrocytic genes, such as COL2A1 and ACAN [20, 27]. Moreover, several studies have revealed the importance of SOX9 in decreasing runt-related transcription factor 2 (RUNX2), osterix (SP7), COL1OA1 and COL1A1 expression, thereby inhibiting early and late osteogenesis [21, 22, 27, 28]. Besides SOX9, SOX5 and SOX6 are also prochondrogenic factors that are essential in the regulation of COL2A1 and other cartilage related genes such as ACAN [29].

Other transcription factors have been identified as important for chondrogenesis. Various studies have revealed that down-regulation or inhibition of forkhead box 03 (FOXO3) [30], zinc finger protein 415 (ZNF145) [31, 32], homeobox D10, D11 and D13 (HOXD10, HOXD11 and HOXD13) [33], decreased the expression of important chondrocyte specific markers during chondrogenic differentiation of MSCs, whereas overexpression of factors such as homeobox A2 and D9 (HOXA2, and HOXD9) increases differentiation of MSCs into chondrocytes [33]. In addition SMADs have been described as important to regulate chondrogenic differentiation of MSCs. Furumatsu et al., demonstrated that SMAD family member 3 (SMAD3) binds to transcription factor SOX9, stimulating chondrogenic differentiation [34], whereas SMAD2 and SMAD3 expression depends on TGFß1 levels during early stages of chondrogenesis [35]. Interestingly, transcription factors SMAD3 and HOXA2 have an inhibitory effect on MSC chondrogenic differentiation by interacting with the SOX9 transcription factor [36]. 
Furthermore, Lui and colleagues showed that overexpression of WNT family member 11 (WNT11) increased chondrogenic differentiation of MSCs by promoting expression of chondrocytic genes [18].

Nongenetic approaches, such as using microenvironmental factors (e.g oxygen conditions), are an attractive alternative due to the safety and easiness to use. Numerous studies have indicated that hypoxia promotes chondrocyte proliferation, survival, and stimulates the expression of gene transcripts to promote cartilage specific ECM formation, suggesting an important role of low oxygen in earlier chondrogenesis, cartilage maintenance and degradation [4, 37, 38]. Previous studies described $S O X 9$ as partly controlled by hypoxia inducible factor $(H I F 1 \alpha)$ during skeletal formation [19], whilst an increased expression of SOX9 has been observed in human articular chondrocytes during low oxygen cultures [39]. Hirao et al. demonstrated that hypoxic conditions also promoted a chondrocyte commitment of cells in the mesenchymal lineage while inhibiting terminal differentiation by activating the transcription of SOX9 in both cells and organ cultures [40]. Moreover, hypoxia inducible transcription factors HIF1 $\alpha$ and HIF2 $\alpha$ were found to be increased during chondrogenic differentiation of MSCs $[39,41,42]$, and were shown to potentiate chondrogenic differentiation by maintaining the chondrocytic phenotype and cell function, while inhibiting hypertrophy and subsequently endochondral ossification in vitro [39]. Hence, hypoxia may be a key factor in tissue engineering for cartilage repair. However, the mechanisms by which articular chondrocytes regulate genes that are sensitive to oxygen levels under physiological conditions remain poorly understood.

During the past decade, multiple studies have demonstrated that miRNAs are involved in the hypoxic response and contribute to the repression of specific genes under low oxygen conditions [45]. MicroRNAs are small non-coding RNA molecules of about 22 nucleotides which principal function is RNA silencing and 
post-transcriptional regulation of gene expression. They frequently repress target genes by reducing the half-life time of their mRNA and/or by inhibiting protein translation. A single miRNA can regulate hundreds of distinct mRNAS simultaneously and a single mRNA can also be regulated by several distinct miRNAS. They contain hypoxia responsive elements (HREs) in their promoter region and are regulated by HIF transcription factors in response to low oxygen [46]. Although HIF1 $\alpha$ is known as a transcriptional activator, it also functions as a transcriptional repressor via induction of miRNAs. Expression of miR-155, miR424, miR-17-92, miR-21, miR-23, miR-24, miR-26, miR-103, miR-107, miR-181 and $m i R-210$ [47-49] were found to be induced under hypoxic conditions and are potential targets of HIF1 $\alpha$. In addition, some of these miRNAs have been implicated in regulating pre and post-natal chondrogenesis. Georgi et al., [50] analyzed miRNA expression in MSCs after 1 week of chondrogenic differentiation and identified $m i R-210$ and $m i R-630$ as positive regulators of chondrogenesis. These two miRNAs were remarkably expressed at higher levels in donors with high chondrogenic potential. In contrast, $m i R-181$ and $m i R-34 a$ were considered as negative regulators of chondrogenesis and their expression was increased in MSCs donors with a low chondrogenic potential [50]. Some other miRNAs that were found to be induced during hypoxia include miR-181a, which is expressed in chondrosarcoma and enhances VEGF expression [51], miR-146a that promotes chondrocytes autophagy and induces cartilage degeneration during osteoarthritis [52], and miR-138 which inhibition helps to maintain a chondrocytic phenotype and reduces the progression of human articular chondrocytes dedifferentiation [53].

In particular $m i R-210$ stands out in several studies as the only hypoxia inducible miRNA $[44,46,47]$ and was found to be essential in the regulation of several biological processes, including cell cycle regulation, cell survival, differentiation, angiogenesis and metabolism, and functions, such as oxidative stress response 
during low oxygen [50, 54, 55]. MiR-210 expression during hypoxic preconditioning of stem cells caused a cytoprotective effect, helping to maintain cell stemness and supporting cell differentiation [56]. This regulation happened in concert with the transcription factor $\operatorname{HIF1} \alpha$ [43].

Despite the efforts, current cell-based repair strategies have been proven unsuccessful for treating large cartilage defects and osteoarthritic lesions. Therefore, proper regeneration of this tissue remains an unresolved question. Our area of investigation will focus its efforts in achieving the understading of proper tissue engineering strategies that could control the commitment and differentiation of the cells while avoiding a hypertrophic phenotype. We will investigate the application of microenviromental conditions to mimic the physiological environment of the cells. Among them, low oxygen tension, which emulates the hypoxic environment of the cells, and the presence of different serum types, such as amongst others synovial fluid, will provide a new insight into the field. Furthermore, the study of a cartilage specific transcription factor will help understanding the specific parameters to create a competent tissue for cartilage repair.

\subsection{AIMS AND OUTLINE OF THE THESIS}

This thesis aims to elucidate the molecular and environmental mechanisms by which we could enhance chondrogenic differentiation of adult mesenchymal stem cells in combination with existing tissue engineering strategies for cartilage regeneration and repair. A better understanding of the use of microenvironmental conditions, such as hypoxia, during differentiation might lead to improvements in differentiation protocols for future stem cell therapies.

Chapter two will provide an overview on the field of cartilage tissue engineering and the use of environmental conditions, such as hypoxia, for cartilage repair and regeneration, including cell sources, growth factors, genetic and mechanical 
stimulation. This chapter will focus as well on the challenges that current strategies present.

Chapter three focuses on validating the molecular gene expression of adipose derived stem cells (aMSCs) during chondrogenic differentiation in low oxygen cultures. It describes aMSC's chondrogenic potential and performance whilst showing their hypertrophic potential when cultured in various 3D- environments and hypoxia.

Chapter four and five draw major attention to the molecular mechanisms by which miRNAs could direct chondrogenesis during the hypoxic response of MSCs in culture conditions. Chapter four draws special attention to the regulation and role of microRNA-210 and its target genes during cartilage development and for the maintenance of healthy cartilage, whereas chapter five explores the potential of microRNA-210 to enhance in vitro chondrogenic differentiation of stem cells.

Chapter six will reveal the effects of synovial fluid on in vitro models of primary chondrocytes and mesenchymal stem cells. Since some concerns have been raised regarding the safety of fetal bovine serum (FBS), the right choice of serum is crucial for cell culture. Particularly with regard to clinical application, human alternatives for FBS are clearly to be preferred. Synovial fluid is commonly present in diseased joints and its interaction with stem cells has not been described. In this chapter, we showed the potential of synovial fluid as a promising novel culture supplement for chondrocyte and aMSCs expansion and chondrogenic differentiation and due to its non-zoonotic (animal free) nature is therefore potentially better for clinical applications in cartilage regeneration.

Chapter seven will explore the role of ZNF648, a cartilage specific transcription factor identified by RNAseq analysis, which is expressed in immature cartilage and growth plate. In vitro cell cultures, functional analysis and a conditional knockout mouse helped us in the understanding of the role of ZNF648 during 
cartilage development. Ultimately, chapter eight will provide a general discussion and overall conclusion of the project. 


\section{REFERENCES}

1. Dudakovic A, Camilleri E, Riester SM, Lewallen EA, Kvasha S, Chen X, Radel DJ, Anderson JM, Nair AA, Evans JM, et al: High-resolution molecular validation of selfrenewal and spontaneous differentiation in clinical-grade adipose-tissue derived human mesenchymal stem cells. J Cell Biochem 2014, 115:1816-1828.

2. $\quad$ Sipp D, Robey P, Turner L: Clear up this stem-cell mess. Nature 2018:455-457.

3. Caplan AI: MSCs: The Sentinel and Safe-Guards of Injury. J Cell Physiol 2016, 231:1413-1416.

4. Galeano-Garces C, Camilleri ET, Riester SM, Dudakovic A, Larson DR, Qu W, Smith J, Dietz AB, Im HJ, Krych AJ, van Wijnen AJ: Molecular Validation of Chondrogenic Differentiation and Hypoxia Responsiveness of Platelet-Lysate Expanded Adipose TissueDerived Human Mesenchymal Stromal Cells. Cartilage 2017, 8:283-299.

5. Iturriaga L, Hernandez-Moya R, Erezuma I, Dolatshahi-Pirouz A, Orive G: Advances in stem cell therapy for cartilage regeneration in osteoarthritis. Expert Opin Biol Ther 2018, 18:883-896.

6. Szychlinska MA, Stoddart MJ, D'Amora U, Ambrosio L, Alini M, Musumeci G: Mesenchymal Stem Cell-Based Cartilage Regeneration Approach and Cell Senescence: Can We Manipulate Cell Aging and Function? Tissue Eng Part B Rev 2017, 23:529-539.

7. Dudakovic A, Camilleri ET, Riester SM, Lewallen EA, Kvasha S, Chen X, Radel DJ, Anderson J, Evans J, Krych AJ, Smith J, Deyle DR, Stein Jl, Stein G, Im HJ, Cool SM, Westendorf J, Kakar S, Dietz AB, van Wijnen AJ: High resolution molecular validation of self-renewal and spontaneous differentiation in clinical grade adipose derived tissue derived human mesenchymal stem cells. J Cell Biochem 2014, 10:1816-1828.

8. Mora JC, Cruz-Almeida Y: Knee osteoarthritis: pathophysiology and current treatment modalities. J Pain Res 2018:2189-2196.

9. Zhu XM, Wang YX, Leung KC, Lee SF, Zhao F, Wang DW, Lai JM, Wan C, Cheng CH, Ahuja AT: Enhanced cellular uptake of aminosilane-coated superparamagnetic iron oxide nanoparticles in mammalian cell lines. Int J Nanomedicine 2012, 7:953-964.

10. Havlas V, Kautzner J, Kaplan A: Arthroscopic technique using crossed K-wires for avulsion fractures of intercondylar eminence in children. Acta Chir Orthop Traumatol Czech 2011, 78:343-347.

11. Paradise CR, Galeano-Garces C, Galeano-Garces D, Dudakovic A, Milbrandt TA, Saris DBF, Krych AJ, Karperien M, Ferguson GB, Evseenko D, van Wijnen AJ: Molecular characterization of physis tissue by RNA sequencing. Gene 2018, 668:87-96.

12. Al-Yamani A, Ahmed F, Abbas M, Sait KHW, Anfinan N, Al-Wasiyah MK, Huwaut EA, Gari M, Al-Qahtani M: Evaluation of in vitro chondrocytic differentiation: A stem cell research initiative at the King Abdulaziz University, Kingdom of Saudi Arabia. BioInformation 2018, 2:53-59.

13. Iturriaga L, Erezuma I, Dolatshahi-Pirouz A, Orive G: Advances in stem cell therapy for cartilage regeneration in osteoarthritis. Expert Opin Biol Ther 2018, 8:883896.

14. Sakata R, Reddi AH: Regeneration of articular cartilage surface: morphogens, cells and extracellular matrix scaffolds. Tissue Eng Part B Rev 2015, 5:461-473.

15. Sakata R, Iwakura T, Reddi AH: Regeneration of Articular Cartilage Surface: Morphogens, Cells, and Extracellular Matrix Scaffolds. Tissue Eng Part B Rev 2015, 21:461-473. 
16. Lu CW, Hu YY, Bai JP, Liu J, Meng GL, Lu R: Three dimensional induction of autologous mesenchymal stem cell and the effects on depressing long-term degeneration of tissue-engineering cartilage. 2007, 45:1717-1721.

17. Liu S, Hou KD, Yuan M, Peng J, Zhang L, Sui X, Zhao B, Xu W, Wang A, Lu S, Guo Q: Characteristics of mesenchymal stem cells derived from Wharton's jelly of human umbilical cord and for fabrication of non-scaffold tissue-engineered cartilage. J Biosci Bioeng 2014, 117:229-235.

18. Ng F, Boucher S, Koh S, Sastry KS, Chase L, Lakshmipathy U, Choong C, Yang Z, Vemuri MC, Rao MS, Tanavde V: PDGF, TGF-beta, and FGF signaling is important for differentiation and growth of mesenchymal stem cells (MSCs): transcriptional profiling can identify markers and signaling pathways important in differentiation of MSCs into adipogenic, chondrogenic, and osteogenic lineages. Blood 2008, 112:295-307.

19. Bridgewater LC, Lefebvre V, de Crombrugghe B: Chondrocyte-specific enhancer elements in the Col11a2 gene resemble the Col2a1 tissue-specific enhancer. J Biol Chem 1998, 273:14998-15006.

20. Kou I, Ikegawa S: SOX9-dependent and independent transcriptional regulation of human cartilage link protein. J Biol Chem 2004, 279:50942-50948.

21. Lefebvre V, Huang W, Harley VR, Goodfellow PN, de Crombrugghe B: SOX9 is a potent activator of the chondrocyte-specific enhancer of the pro alpha1(II) collagen gene. Mol Cell Biol 1997, 17:2336-2346.

22. Sekiya I, Tsuji K, Koopman P, Watanabe H, Yamada Y, Shinomiya K, Nifuji A, Noda M: SOX9 enhances aggrecan gene promoter/enhancer activity and is up-regulated by retinoic acid in a cartilage-derived cell line, TC6. J Biol Chem 2000, 275:10738-10744.

23. Zhang P, Jimenez SA, Stokes DG: Regulation of human COL9A1 gene expression. Activation of the proximal promoter region by SOX9. J Biol Chem 2003, 278:117-123.

24. Augello A, De Bari C: The regulation of differentiation in mesenchymal stem cells. Hum Gene Ther 2010, 21:1226-1238.

25. Yang B, Guo H, Zhang Y, Chen L, Ying D, Dong S: MicroRNA-145 regulates chondrogenic differentiation of mesenchymal stem cells by targeting Sox9. PLoS One 2011, 6:e21679.

26. Wang ZH, Li XL, He XJ, Wu BJ, Xu M, Chang HM, Zhang XH, Xing Z, Jing XH, Kong $\mathrm{DM}$, et al: Delivery of the Sox 9 gene promotes chondrogenic differentiation of human umbilical cord blood-derived mesenchymal stem cells in an in vitro model. Braz J Med Biol Res 2014, 47:279-286.

27. Guerit D, Philipot D, Chuchana P, Toupet K, Brondello JM, Mathieu M, Jorgensen C, Noel D: Sox9-regulated miRNA-574-3p inhibits chondrogenic differentiation of mesenchymal stem cells. PLoS One 2013, 8:e62582.

28. Park JS, Yang HN, Woo DG, Jeon SY, Do HJ, Lim HY, Kim JH, Park KH: Chondrogenesis of human mesenchymal stem cells mediated by the combination of SOX trio SOX5, 6, and 9 genes complexed with PEI-modified PLGA nanoparticles. Biomaterials 2011, 32:3679-3688.

29. Guerit D, Brondello JM, Chuchana P, Philipot D, Toupet K, Bony C, Jorgensen C, Noel D: FOX03A regulation by miRNA-29a Controls chondrogenic differentiation of mesenchymal stem cells and cartilage formation. Stem Cells Dev 2014, 23:1195-1205.

30. Liu TM, Martina M, Hutmacher DW, Hui JH, Lee EH, Lim B: Identification of common pathways mediating differentiation of bone marrow- and adipose tissue-derived 
human mesenchymal stem cells into three mesenchymal lineages. Stem Cells 2007, 25:750-760.

31. Liu TM, Guo XM, Tan HS, Hui JH, Lim B, Lee EH: Zinc-finger protein 145, acting as an upstream regulator of SOX9, improves the differentiation potential of human mesenchymal stem cells for cartilage regeneration and repair. Arthritis Rheum 2011, 63:2711-2720.

32. Seifert A, Werheid DF, Knapp SM, Tobiasch E: Role of Hox genes in stem cell differentiation. World J Stem Cells 2015, 7:583-595.

33. Furumatsu T, Tsuda M, Yoshida K, Taniguchi N, Ito T, Hashimoto M, Ito T, Asahara H: Sox9 and p300 cooperatively regulate chromatin-mediated transcription. J Biol Chem 2005, 280:35203-35208.

34. Zhang T, Wen F, Wu Y, Goh GS, Ge Z, Tan LP, Hui JH, Yang Z: Cross-talk between TGF-beta/SMAD and integrin signaling pathways in regulating hypertrophy of mesenchymal stem cell chondrogenesis under deferral dynamic compression. Biomaterials 2015, 38:72-85.

35. Almalki SG, Agrawal DK: Key transcription factors in the differentiation of mesenchymal stem cells. Differentiation 2016, 92:41-51.

36. Zhong L, Huang X, Rodrigues ED, Leijten JC, Verrips T, El Khattabi M, Karperien M, Post JN: Endogenous DKK1 and FRZB Regulate Chondrogenesis and Hypertrophy in Three-Dimensional Cultures of Human Chondrocytes and Human Mesenchymal Stem Cells. Stem Cells Dev 2016, 25:1808-1817.

37. Wu L, Leijten JC, Georgi N, Post JN, van Blitterswijk CA, Karperien M: Trophic effects of mesenchymal stem cells increase chondrocyte proliferation and matrix formation. Tissue Eng Part A 2011, 17:1425-1436.

38. Adesida AB, Grady LM, Khan WS, Millward-Sadler SJ, Salter DM, Hardingham TE: Human meniscus cells express hypoxia inducible factor-1alpha and increased SOX9 in response to low oxygen tension in cell aggregate culture. Arthritis Res Ther 2007, 9:R69.

39. Hirao M, Tamai N, Tsumaki N, Yoshikawa H, Myoui A: Oxygen tension regulates chondrocyte differentiation and function during endochondral ossification. J Biol Chem 2006, 281:31079-31092.

40. Zhang FJ, Luo W, Lei GH: Role of HIF-1alpha and HIF-2alpha in osteoarthritis. Joint Bone Spine 2015, 82:144-147.

41. Markway BD, Cho H, Johnstone B: Hypoxia promotes redifferentiation and suppresses markers of hypertrophy and degeneration in both healthy and osteoarthritic chondrocytes. Arthritis Res Ther 2013, 15:R92.

42. Ivan M, Huang X: miR-210: fine-tuning the hypoxic response. Adv Exp Med Biol 2014, 772:205-227.

43. Bertero T, Rezzonico R, Pottier N, Mari B: Impact of MicroRNAs in the Cellular Response to Hypoxia. Int Rev Cell Mol Biol 2017, 333:91-158.

44. Mircea I, Huang X: miR-210: Fine-Tuning the Hypoxic Response. Adv Exp Med biol 2015, 772:205-227.

45. Kulshreshtha R, Ferracin M, Wojcik SE, Garzon R, Alder H, Agosto-Perez FJ, Davuluri R, Liu CG, Croce CM, Negrini M: A microRNA signature of hypoxia. Mol Cell Biol 2007, 27:1859-1867.

46. Huang F, Zhu X, Hu XQ, Fang ZF, Tang L, Lu XL, Zhou SH: Mesenchymal stem cells modified with miR-126 release angiogenic factors and activate Notch ligand Delta-like-4, enhancing ischemic angiogenesis and cell survival. Int J Mol Med 2013, 31:484-492. 
47. Chen W, Cai F, Zhang B, Barekati Z, Zhong XY: The level of circulating miRNA-10b and miRNA-373 in detecting lymph node metastasis of breast cancer: potential biomarkers. Tumour Biol 2013, 34:455-462.

48. Sarkar S, Dey BK, Dutta A: MiR-322/424 and -503 are induced during muscle differentiation and promote cell cycle quiescence and differentiation by down-regulation of Cdc25A. Mol Biol Cell 2010, 21:2138-2149.

49. Georgi N, Taipaleenmaki H, Raiss CC, Groen N, Portalska KJ, van Blitterswijk C, de Boer J, Post JN, van Wijnen AJ, Karperien M: MicroRNA Levels as Prognostic Markers for the Differentiation Potential of Human Mesenchymal Stromal Cell Donors. Stem Cells Dev 2015, 24:1946-1955.

50. Sun X, Wei L, Chen Q, Terek RM: MicroRNA regulates vascular endothelial growth factor expression in chondrosarcoma cells. Clin Orthop Relat Res 2015, 473:907-913.

51. Zhang F, Wang J, Chu J, Yang C, Xiao H, Zhao C, Sun Z, Gao X, Chen G, Han Z: MicroRNA-146a Induced by Hypoxia Promotes Chondrocyte Autophagy through Bcl-2. Cell Physiol Biochem 2015, 37:1442-1453.

52. Seidl CI, Martinez-Sanchez A, Murphy CL: Expression of MicroRNA-138 Contributes to Loss of the Human Articular Chondrocyte Phenotype. Arthritis Rheumatol 2016, 68:398-409.

53. Chan SY, Loscalzo J: MicroRNA-210: a unique and pleiotropic hypoxamir. Cell Cycle 2010, 9:1072-1083.

54. Chan YC, Banerjee J, Choi SY, Sen CK: miR-210: the master hypoxamir. Microcirculation 2012, 19:215-223.

55. Pei M: Environmental preconditioning rejuvenates adult stem cells' proliferation and chondrogenic potential. Biomaterials 2017, 117:10-23. 


\section{CHAPTER 2}

2. Hypoxia regulatory mechanisms

during cartilage development and cartilage-tissue engineering. 


\subsection{INTRODUCTION}

Articular cartilage (AC) is the connective tissue found at the ends of diarthrodial joints and is adapted to provide lubrication during motion [1]. During early stages of limb development, the vasculature is subjected to an extensive remodeling process leaving the prechondrogenic condensation avascular [2]. Due to this avascular environment, AC is formed and maintained in a hypoxic environment during development into adulthood [3], and functions from this stage onwards at an oxygen tension that is lower than other tissues. It has been determined that the oxygen supply at the articular surface is approximately 6 to $10 \%$, whereas in the deepest layers of AC oxygen supply is no more than 1 to $6 \%$ of oxygen [4]. Cramer et al. provided evidence that the growth plate is also characterized by a hypoxic microenvironment during fetal development [5]. The upper hypertrophic zone and the central portion of the proliferative zone in the growth plate are exposed to low oxygen tensions [6], suggesting that in physiological conditions chondrocytes depend on diffusion from the epiphyseal and subchondral bone to rely on nutrient supply [7].

Chondrocytes are responsible for the synthesis and maintenance of its extra cellular matrix, which gives the articular cartilage its mechanical integrity for the daily load-bearing conditions [8]. Chondrocytes are able to survive in a sustained low oxygen environment. Thus, it has been hypothesized that hypoxia is an important factor in regulating growth and survival of chondrocytes, while it has been suggested as a key factor to conserve cartilage integrity [9]. A number of pathophysiological findings propose that a correlation does exist between hypoxia and chondrogenesis [10]. Additionally, several studies sustain that a low oxygen tension environment may be beneficial for the maintenance of the chondrocyte phenotype in vitro, avoiding potential dedifferentiation during cell culture and expansion [11]. However, the exact mechanism by which chondrocytes are regulated by oxygen tension under normal conditions remains 
poorly understood. In this chapter we will present an overview of the hypoxia regulatory mechanisms and their role during cartilage development and cartilage tissue engineering.

\subsection{THE HYPOXIC PATHWAY}

The process of oxygen regulation is mostly coordinated by the HIF (HypoxiaInducible Factor) pathway. HIF is a basic helix-loop-helix transcription factor that has two main roles. The first role is to transactivate gene-encoding proteins that participate in homeostatic responses. The second role involves the modulation of multiple key metabolic pathways for the maintenance of oxygen homeostasis [12]. HIF is a heterodimer of bHLH-PAS proteins and consists of an unstable alpha subunit and a stable beta subunit. These bind DNA to the hypoxia response elements (HREs), which are present in the promoter region of target genes to activate their expression [13]. Three HIF isoforms are known: HIF1 $\alpha$, HIF $2 \alpha$ and HIF $\alpha$ [14]. These isoforms have structural similarities but distinct roles. This conservation of the HIF genes across different species suggests that they perform essential non-overlapping functions during the hypoxic response [6] in both humans and animals [15].

In mammals, the three genes that encode HIF $\alpha$ subunits appear to be regulated in a similar manner [16]. The HIF1 $\alpha$ protein is ubiquitously expressed [17], whereas its homologs, HIF2 $\alpha /$ Endothelial PAS domain protein (EPAS) [16] and HIF3 $\alpha$ [18], have more restricted expression patterns. HIF $\beta$ is generally found to be constitutively expressed and insensitive to changes in oxygen availability [19]. Although there is evidence for hypoxic induction of $H I F \alpha$ mRNA levels in some cell types [20], the predominant modes of $\mathrm{HIF} \alpha$ regulation occur posttranslationally. Under normal oxygen conditions, hypoxia inducible factor $\alpha$ subunits HIF1 $\alpha$ and HIF $2 \alpha$ are hydroxylated by prolyl hydroxylase domain (PHD) enzymes at specific proline residues (Figure 2.1.A), then they are ubiquitinated through interaction with the von Hippel-Lindau tumor suppressor protein 
(pVHL) (Figure 2.1.B), and subsequently degraded by the $26 \mathrm{~S}$ proteasomal pathway in the cytosol, resulting in minimal transcriptional activity (Figure 2.1.C). Under hypoxia, the activity of the HIF-targeting prolyl hydroxylase enzymes is inhibited, allowing HIF $1 \alpha / 2 \alpha$ to rapidly accumulate in the cytoplasm (Figure 2.1.D). Then, HIF $1 \alpha / 2 \alpha$ gets phosphorylated, translocated into the nucleus (Figure 2.1.E and F), and dimerized with HIF1 $\beta$ in order to activate transcription of its target genes.

Low oxygen has the capability of influence cell signals and functions [21]. Many of the factors that increase during hypoxia are regulated by HIF activation. Transcriptional selectivity studies have defined exclusive targets for each of the isoforms while defining genes that are responsive to some of them [22]. Around 100 HIF targets have been described in the literature [23]. HIF-dependent transcription activates genes involved in cell autonomous development pathways including hematopoietic, endothelial, myocyte, adipocyte, chondrocyte, trophoblast, and neuronal differentiation programs, and various physiological activities such as erythropoiesis, angiogenesis, autophagy and energy metabolism [12, 24-26].

It is believed that HIF factors are involved in chondrocyte development and in the regulation of cartilage homeostasis [17, 18, 20]. HIF1 $\alpha$ and HIF2 $\alpha$ have been the most extensively studied. Both genes have shown to be involved in promoting chondrogenesis and to play an active role in chondrocyte development [15]. Both HIF1 $\alpha$ and HIF2 $\alpha$ play an important role in the activation of hypoxia responsive genes and could activate important pathways for cartilage homeostasis and repair. Given the importance of HIF in maintaining oxygen homeostasis and essential functions in humans, it is expected that HIF perturbation would disrupt the most basic developmental processes. Lyer et al. observed that inactivation of HIF causes severe developmental abnormalities and impairment of joint development [27]. 


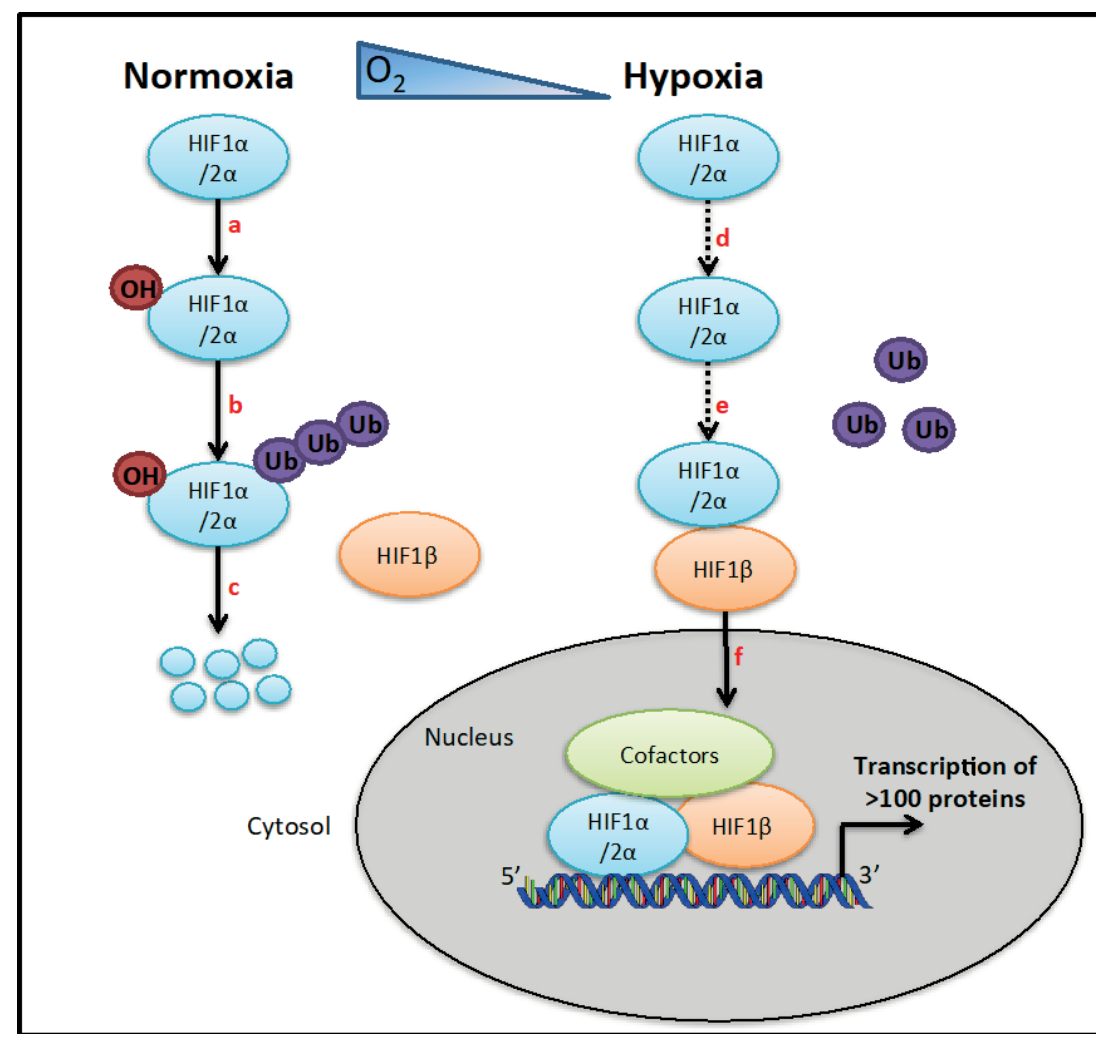

Figure 2.1 Hypoxia pathway regulation in normoxia and hypoxia. (A) In normoxia, hypoxia inducible factor $\alpha$ subunits HIF1 $\alpha$ and HIF $2 \alpha$ are hydroxylated by PHD, (B) then it is ubiquitinated through interaction with pVHL, and (C) subsequently degraded by the 26S proteasomal pathway in the cytosol resulting in minimal transcriptional activity. (D) In hypoxia, the activity of the HIFtargeting prolyl hydroxylase enzymes is inhibited allowing HIF $1 \alpha / 2 \alpha$ to rapidly accumulate in the

cytoplasm. (e and f) Then, HIF1 $\alpha / 2 \alpha$ gets phosphorylated, translocated into the nucleus and dimerized with HIF1 $\beta$ in order to activate transcription of its target genes.

\subsubsection{HIF $1 \alpha$}

HIF1 $\alpha$ is the most important factor involved in the cellular response to low oxygen and is known as the master regulator of oxygen homeostasis [28]. HIF1 $\alpha$ acts as a transcription factor and activates the transcription of genes (Figure 2.1). Interestingly, HIF1 $\alpha$ has been identified as a key mediator for chondrocytes to respond to fluctuations of oxygen availability during cartilage development and repair, and may serve as a target for modulating chondrocyte functions [29]. 
Fernández-Torres et al. have shown that HIF1 $\alpha$ gene plays a protective role against joint damage and might have a beneficial effect in maintaining cartilage homeostasis [30]. Schipani et al. revealed that cartilage structures in the limbs of mouse embryos are highly hypoxic and that HIF1 $\alpha$ is essential for accurate growth-plate and joint formation [6]. Moreover, Mackie et al. revealed that $H I F 1 \alpha$ could act synergistically with $B M P 2$ to promote the expansion and proliferation of chondrocytes while inhibiting hypertrophic differentiation and subsequently endochondral ossification [14]. A need of HIF1 $\alpha$ transcription factor for proper chondrocyte function and cartilage homeostasis was clear in mice with an inactivation of HIF1 $\alpha$ in cartilaginous structures. A decreased in collagen type II and aggrecan, and cartilage degeneration was observed in Hif1 $\alpha$ KO mouse when compared to wild type [31]. Nevertheless, no studies have stablished the necessity of HIF1 $\alpha$ during cartilage formation.

\subsubsection{HIF $2 \alpha$}

Hypoxia-inducible factor 2 (HIF2) is a heterodimer of two proteins, HIF2 $\alpha$ and HIF1 $\beta$ [32]. HIF2 $\alpha$ is regulated via oxygen-dependent post-translational degradation and involved in controlling hypoxic responses through activation of target genes [8] (Figure 2.1). Although HIF1 $\alpha$ and HIF2 $\alpha$ show different sensitivity to oxygen tension and display distinct cellular activities, HIF $2 \alpha$ helps HIF $1 \alpha$ to mediate the response of cells to hypoxia [10]. In recent studies, HIF2 $\alpha$ has emerged as the primary HIF involved in chondrocyte differentiation and its importance in articular cartilage homeostasis has been reported [32]. Lafont et al. provided evidence that HIF $2 \alpha$ is essential for hypoxic induction of the human articular chondrocyte phenotype and promotes cartilage-matrix synthesis through induction of key cartilage genes [8]. Since it is highly expressed in degenerated cartilage diseases such as osteoarthritis (OA) and rheumatoid arthritis (RA) in humans and mice, it also appears to be implicated in catabolic mechanisms leading to cartilage breakdown and endochondral bone formation 
[33]. Furthermore, a Hif2 $\alpha$-deficient mouse showed a multi organ failure and oxidative damage, which demonstrated the importance of this protein in tissue development and oxygen homeostasis [28].

\subsubsection{HIF $3 \alpha$}

Hypoxia Inducible Factor 3 Alpha Subunit (HIF3 $\alpha$ ) is a protein-coding gene that acts as a regulator of hypoxia-inducible gene expression [34]. Although HIF3 $\alpha$ functions remain largely unexplored, available data on HIF3 $\alpha$ indicate that this hypoxia inducible factor functionality differs from HIF1 $\alpha$ and HIF $2 \alpha$ [12]. HIF3 $\alpha$ is induced at the protein level by hypoxia and regulated by HIF $\alpha$ and HIF $2 \alpha$ at the transcriptional level [35]. Yet, HIF3 $\alpha$ could act as negative regulator of HIF1 $\alpha$ and HIF2 $\alpha$ [19]. Recent studies have described that HIF3 $\alpha$ gene expression was lower in OA chondrocytes than in healthy chondrocytes. A lower HIF3 $\alpha$ gene expression was found in the hypertrophic zone of human embryonic epiphyseal tissue, suggesting that $H I F 3 \alpha$ is a negative regulator of hypertrophic differentiation [35]. However, HIF3 $\alpha$ 's role in chondrocyte development and phenotype has not been studied in much detail. Overall, the previous data indicate manipulation of the HIF pathway during chondrogenic differentiation in culture could promote cartilage regeneration and repair [6].

\subsection{HYPOXIA DURING SKELETAL FORMATION IN MAMMALS}

Chondrogenesis and endochondral ossification are cartilage differentiation processes that lead to skeletal formation and growth. Both processes are instrumental in skeletal repair after trauma [32]. In endochondral ossification, chondrocytes undergo well-ordered and controlled phases of proliferation, hypertrophic differentiation, mineralization of the surrounding matrix, death, blood vessel invasion and finally replacement of cartilaginous matrix with bone [6]. This process of chondrocytic matrix replacement by bone tissue is a welldefined temporal and spatial sequence of events and it is impacted by a number of different types of matrix molecules, growth factors, microenvironmental 
conditions and stress [37]. As a principal factor during this process of endochondral ossification, vascular endothelial growth factor (VEGF), an angiogenic promoter protein, acts as a coordinator of chondrocyte death and extracellular matrix remodeling by functional activation of chondrocytes, chondroclasts and osteoblasts and attracting the ingrowth of blood vessel [5]. Moreover, hypertrophic chondrocytes synthesize type X collagen and mineralize their surrounding matrix before being replaced by bone tissue [37].

Due to the cartilage avascular nature, it relies on diffusion to obtain critical nutrients, such as oxygen and glucose. Oxygen distributes from the surrounding synovial fluid into the tissue, creating an oxygen gradient in the cartilage [38]. Zhou et al. created a mathematical model to calculate oxygen levels in human articular cartilage. This model proposed that oxygen levels may range from $5 \%$ on the articular surface to $1 \%$ in the deep zone [39]. This oxygen gradient depends on several factors, including oxygen concentration in synovial fluid, cartilage thickness and cell density, with oxygen tension decreasing with distance from the cartilage surface. In vivo measurements exhibited that oxygen tension in human articular cartilage ranges from $7 \%$ (53 $\mathrm{mm} \mathrm{Hg}$ ) in the superficial layer to less than $1 \%$ (7.6 $\mathrm{mm} \mathrm{Hg}$ ) in the deep zone [4]. Moreover, calculated oxygen tensions fell with increasing distance from the synovial surface to $\sim 2 \%$ in the deep zone of bovine knee cartilage and to $3.6 \%$ for bovine ankle cartilage and dog knee, but remained above 5\% for rabbit knee cartilage [39]. Furthermore, Brighton et al. described oxygen measurements on in vivo preparations of tibial epiphyses of rabbits and rats [3]. The proximal tibial epiphyses exhibited a low oxygen tension of approximately 19.5 millimeters of mercury in the resting zone whilst increasing progressively to a high tension of approximately 95.2 millimeters of mercury in the metaphyseal bone. It is clear that oxygen tension within healthy cartilage will differ from joint to joint and from species to species. 
However, in all species oxygen tension decreases from the cartilage surface to deeper zones to an estimate of around 1\% of oxygen [39].

It has been suggested that low oxygen maintains cartilage homeostasis by inhibiting angiogenesis and hypertrophic differentiation by reducing VEGF and COL10A1 expression while inducing several genes required for matrix production such as glycosaminoglycans [40] and COL2A1 during chondrocyte development [41]. Oxygen tension can have significant effects on the metabolism of articular cartilage, including changes in proteoglycan synthesis [42, 43]. Kuiper et al. suggested that chondrocytes synthesize more sulphated glycosaminoglycans (GAGs) when deeper in the tissue in conditions of chronic hypoxia [44]. Culturing cartilage at $5 \% \mathrm{O}_{2}$ significantly increased proteoglycan and collagen synthesis compared with $20 \% \mathrm{O}_{2}$ [42]. However, cartilage cultured at $1 \% \mathrm{O}_{2}$ caused a significant decrease in proteoglycan and collagen synthesis compared with $20 \%$ $\mathrm{O}_{2}$. Also, an increased in hyaluronan synthesis occurred after cells were culture

for 12 hours at $5 \% \mathrm{O}_{2}$ compared to $20 \% \mathrm{O}_{2}$, but decreased at $1 \% \mathrm{O}_{2}$ compared with $20 \% \mathrm{O}_{2}$ [45]. An adequate balance in oxygen tension is still necessary to achieve proper chondrogenesis for cartilage regeneration strategies.

\subsection{CROSSTALK BETWEEN HYPOXIA AND CARTILAGE RELATED PATHWAYS}

Gene expression and translation of hundreds of genes and proteins have been described to be oxygen dependent and to respond in helping chondrocytes during various physiological processes such as proliferation, cell fate, senescence and apoptosis [15]. Several studies have unravelled a complex molecular network that is involved in regulating the hypoxic response in physiological conditions [46]. These include various signaling pathways, fibroblast growth factors (FGFs), transforming growth factor $\beta(\mathrm{TGF} \beta)$, bone morphogenic proteins (BMPs), WNT, $\beta$-catenin, Hedgehog $(\mathrm{HH})$, and Notch signaling pathways. 
In native cartilage, growth factors stimulate cell growth, proliferation, differentiation and apoptosis [47]. Moreover, these specific biomolecules are strongly related to tissue damaged repair. Thus, growth factors are largely used in tissue regeneration approaches. For cartilage repair strategies, the most used biomolecules are those who act to initiate or suppress signaling molecules cascades during chondrogenesis. TGF $\beta$, BMP and IGF families are the most extensively studied developmental morphogens to stimulate chondrogenic differentiation of stem cells in vitro [48]. In addition, WNT, NOTCH and FGF are interesting candidates based on their in vivo roles during cartilage homeostasis [49]. Various members of these families have been tested in vitro as chondrogenic inducers and in combination with tri-dimensional environments, mechanical loading, genetic approaches and hypoxia [50]. Stem cells and chondrocytes closely interact with the biochemical and environmental stimuli during chondrogenesis to maintain a normal chondrocyte phenotype and avoid hypertrophic differentiation. Moreover, these biomolecules closely interact with the hypoxia pathway to activate lineage specific transcription factors such as SOX9. SOX9 signaling helps to maintain the chondrocytic phenotype by inhibiting RUNX2 expression preventing hypertrophy and posterior endochondral ossification [14]. In this section we will define how these signals, in combination with low oxygen tension, enhance in vitro chondrogenic differentiation of stem cell cultures for cartilage tissue engineering.

\subsubsection{WNT signaling}

WNT signaling has been previously described as a key factor during cartilage homeostasis. It participates in a tightly regulated and highly specific regulatory process of chondrogenic differentiation in progenitor cells [15]. Specifically, WNT activity is required for proliferation and maintenance of the chondrocytic phenotype [14]. In the case of WNT3a and WNT5b they promote chondrogenesis while delaying hypertrophy. WNT11 overexpression in MSCs during 
chondrogenic differentiation promotes a chondrocytic phenotype but also upregulates RUNX2 and indian hedgehog (IHH) expression, two genes involved in hypertrophic differentiation [15]. WNT1, WNT4, WNT7a, WNT8 and WNT9 have shown to inhibit chondrocyte differentiation and were found increased during OA progression $[52,53]$.

Overexpression of WNT signalling can increase chondrocyte hypertrophy and expression of degrading metalloproteinases in cartilage. Unmarino et al. mentioned that new research links the degradation of articular cartilage and OA progression to the interaction of $H I F 1 \alpha$ with the WNT signaling pathway [54]. Interestingly, HIF1 $\alpha$ increases the expression of WNT signaling inhibitors Dickkopf (DKK1), frizzled B (FRZB) and Gremlin 1 (GREM1), which decreased the expression of hypertrophic markers during chondrogenesis of MSCs and prevented cartilage loss $[87,133,134]$. Moreover, the presence of WNT signaling inhibitors increased the expression of collagen type II and aggrecan. Therefore, regulation of the WNT signaling pathway by hypoxia and the HIF signaling pathway is fundamental to control both cartilage maintenance and prevention of OA progression.

\subsubsection{Wnt inducible signaling proteins (WISPs)}

WNT inducible signaling proteins (WISPs) are highly expressed in skeletal tissue and osteoprogenitor cells [55]. These proteins are known to participate in the intention to restore damaged articular cartilage during subchondral bone development. They have an important role in the formation, growth, differentiation and maintenance of both bone and cartilage [29]. An upregulation of WNT signaling pathway and WISP1 during OA progression increased the expression of matrix metalloproteinases (MMPs), interleukins and aggrecanases. Therefore, inhibition of WISP1 signaling is essential for cartilage preservation. In Chapter 4 using various target prediction software's, we show that WISP1 is a potential target of miR-210 during the hypoxia response. 


\subsubsection{2. $\beta$-catenin}

Regulation of $\beta$-catenin levels is important for the homeostasis of articular chondrocytes. Excessive or insufficient $\beta$-catenin will enhance pathological maturation at the periphery of the cartilage and early apoptosis at the center. The interaction between HIF1 $\alpha$ and $\beta$-catenin has been described as a negative regulator of WNT signaling and promotes a good balance in cartilage homeostasis and chondrocyte stability [55]. In contrast, HIF2 $\alpha$ positively regulates $\mathrm{WNT} / \beta$-catenin and interacts with $\mathrm{NF}-\mathrm{\kappa B}$ pathways to promote chondrocyte hypertrophy, apoptosis and endochondral ossification [33].

\subsubsection{Transforming growth factor beta (TGF $\beta$ )}

Members of the TGF $\beta$ superfamily are growth factors known to be essential for multiple stages of embryonic chondrogenesis [15]. They participate in stimulating differentiation of chondroprogenitor cells into chondrocytes while inhibiting chondrocyte hypertrophy and maturation. TGF $\beta$ family members, such as TGF $\beta 1,2,3$, and BMPs, are known as major regulators in chondrogenesis and osteogenesis [14]. These factors serve as stimuli to promote growth and in vitro chondrogenic differentiation of MSCs [6]. However, in vitro administration of TGF $\beta 1$ has been shown to redirect human articular chondrocytes towards hypertrophy [15]. Several pro-chondrogenic genes that are regulated by TGF $\beta$ require also HIF1 $\alpha$ expression. Ueno et al. demonstrated that silencing HIF1 $\alpha$ could attenuate TGF $\beta 1$ induced chondrogenic gene expression, whereas SanchezElsner et al., confirmed that low oxygen mediates an interaction between HIF1 $\alpha$ and $T G F \beta$ via SMAD3/4 signaling pathway $[56,57]$. Hence, stimulation of chondrocytes with TGF $\beta$ could require HIF1 $\alpha$ protein accumulation.

\subsubsection{Bone morphogenetic protein (BMP)}

Bone Morphogenetic proteins (BMPs) are cytokines that belong to the transforming growth factor- $\beta$ (TGF $\beta$ ) superfamily [15]. They are essential for mesenchymal condensastion and chondrogenic differentiation of mesenchymal 
stem cells (MSCs) [14]. BMP2 has shown to stimulate COL2A1 gene expression in human articular chondrocytes. Moreover, BMP2 and BMP4 have been shown to induce hypertrophy in progenitor cells, whereas BMP7 maintains a chondrogenic potential while preventing chondrocyte hypertrophy [8] and terminal differentiation [58].

\subsubsection{Insulin Like Growth Factor (IGF)}

IGFs are single-chain polypeptides that have similar function and structure to insulin. These hormones are involved in mediating growth and development. IGF1 is a small protein that regulates growth in adults and plays a main role in DNA synthesis in multiple cell types, including chondrocytes. It penetrates into articular cartilage through the synovial fluid to stimulate chondrocyte proliferation and proteoglycan homeostasis [59]. In recent years, addition of IGF1 has shown to stimulate the chondrogenic potential of stem cells. As observed, IGF1 stimulates proteoglycan production while promoting the maintenance of the chondrogenic phenotype of articular chondrocytes. In addition, it has been demonstrated that a combination of IGF1 with either BMP7 or TGF $\beta 1$ results in greater repair potential when compared with either factor alone [60].

\subsubsection{Fibroblast Growth Factor (FGF)}

FGFs are known to be involved during cartilage development [15]. Progression of chondrocyte differentiation and maturation has been associated with FGF2, FGF9, and FGF18 expression [6]. FGF2 has the function of priming cells for chondrocytic differentiation whilst enhancing chondrocyte proliferation $[6,60]$. Previous studies have shown that interaction of FGF2 with TGF $\beta$ and WNTs enhances chondrogenesis [15]. In addition, FGF9 and FGF18 are known regulators of early chondrogenic differentiation and are involved in augmenting ECM production as a result of gene expression up-regulation of pro-chondrogenic proteins such as SOX9, IHH and COL2A1. In a rat model of osteoarthritis, Fgf18 acted as a pro38 
chondrogenic factor and was shown to stimulate repair of damaged cartilage [15]. Interestingly, the interaction of HIF1 $\alpha$ and FGF correlated to early chondrogenesis and chondrogenic differentiation [135].

\subsubsection{Hedgehog signaling}

Sonic hedgehog (SHH) signaling is critically important during early chondrogenesis due to its role in controlling cell fate and its synergism with other pro-chondrogenic factors. It acts synergistically with BMP signaling to increase SOX9 expression while interacting with the FGF family to enhance chondrogenesis in the earliest stages of endochondral ossification [15]. IHH is synthesized during pre-hypertrophic and hypertrophic stages of chondrocytes in the growth plate. It has shown to stimulate chondrocyte proliferation and hypertrophy, while inducing differentiation of perichondrial progenitor cells into osteoblasts to form the collarbone [62]. Although IHH is not considered one of the master regulators of chondrogenesis, previous studies showed that IHH could be as potent as TGF $\beta 1$ and BMP2 in chondrogenic induction of primary MSCs [136]. Precise homeostatic regulation of $I H H$ is crucial for promoting chondrogenesis over osteogenesis. Bijlsma et al. demonstrated that hypoxia is able to induce a hedgehog response in adult mice $[63,64]$. In vitro models of adult stem cells showed that accumulation of $H I F 1 \alpha$ was sufficient to secrete $S H H$ and hedgehog signaling. In contrast, pharmacological inhibition and knockdown of HIF1 $\alpha$ in ischaemic adult tissue inhibited $S H H$ and hedgehog pathway activation. Thus, induction of hedgehog signaling can be controlled through HIF1 $\alpha$ accumulation after exposure to low oxygen [65]. Altogether, hedgehog factors are important pro-chondrogenic signaling factors that could have significant impact in cartilage tissue engineering.

\subsection{HYPOXIA AND CARTILAGE DESTRUCTION}

Since oxygen tension is altered in arthritic joints [65], the oxygen conditions during $\mathrm{OA}$ development have been largely studied. A depletion of oxygen in 
patients with $\mathrm{OA}$ has been reported to be due to alterations in blood flow, gas exchange and inflammatory factors in the synovial membrane and subchondral bone [6]. Several groups have reported an increase in HIF1 $\alpha$ and HIF2 $\alpha$ transcriptional activity in OA cartilage and OA chondrocytes, particularly in the late stages of disease [33]. HIF1 $\alpha$ is up-regulated by other factors, such as inflammatory cytokines, mechanical loading and reactive oxygen species, which are key factors for the regulation of cartilage degradation. In addition, Houard et al. found that $H I F 2 \alpha$ is a modulator of matrix degradation in articular cartilage during OA progression. They indicated that HIF2 $\alpha$ could potentiate IL6 and TNF $\alpha$ signaling in diseased chondrocytes [68]. Moreover, a number of catabolic enzymes associated with OA progression have been identified as direct HIF2 $\alpha$ targets [69]. Although, maintaining a hypoxic environment is essential for cartilage homeostasis, the imbalance in oxygen tension during cartilage degeneration in osteoarthritis (OA) and rheumatoid arthritis (RA) progression increases cartilage degradation, increasing inflammation and metalloproteinase expression, while decreasing proteoglycan deposition.

Osteoarthritis is a degenerative joint disease that predominantly induces mechanical stress changes in cartilage and bone [29]. OA constitutes a major health problem that is responsible for chronic disability and impacts the quality of life of millions of people worldwide [39]. Several cytokines and inflammatory markers have been found elevated during OA progression, including RUNX2, COL10A1, IHH, alkaline phosphatase (ALPL), MMP13, interleukin $1 \beta$ (IL1 $\beta$ ) and tumor necrosis factor alpha (TNF $\alpha$ ), which are all closely associated with cartilage degradation $[39,66]$. IL1 $\beta$ and TNF $\alpha$ have been largely measured in synovial fluid during cartilage $\mathrm{OA}$ and their concentrations are highly dependent on the stage of disease [68].

Rheumatoid arthritis is a polyarticular chronic inflammatory autoimmune disease that largely affects the joints and is associated with cartilage destruction 
and functional disability. During the development of RA, cartilage is destroyed by pro-inflammatory cytokines, such as TNF $\alpha$ with additional roles from IL6 and IL1 $\beta$. These cytokines are mainly derived from the synovial membrane or cartilage and accumulate in the synovial fluid. They are known to contribute to joint inflammation and cartilage destruction by increasing expression of matrix degrading enzymes [39].

Degeneration of articular cartilage has been associated to several factors that predispose the tissue towards disease. Among these factors are aging, injuries, joint instability, mechanical stress, inflammation and metabolic disorders [39, 66]. The stress generated by these factors often leads to a disruption in the balance of cartilage anabolic and catabolic processes, which can result in chondrocytes phenotypic change, cell death and an increase in inflammatory genes [65]. Ultimately, all these changes will lead to deterioration of structural and functional properties of the cartilage and joint destruction [66]. Although there is a broad range of stress factors that contribute to the development of arthritis, the exact mechanism of its pathogenesis is still unknown $[137,138]$.

\subsection{HYPOXIA AND TISSUE ENGINEERING}

Over the past decade, tissue engineering has become the prospective solution for defects resulting from severe trauma, osteodegenerative diseases and mechanical stress. Significant research on this field has been made by engineers and physicians [15], which have described different methods to restore joint function and prevent joint degeneration. To achieve a proper tissue engineering strategy for regeneration of cartilage, several characteristics are needed: 1) an appropriate cell source with the ability to proliferate, differentiate and maintain a chondrocytic phenotype over a long time period, 2) a proper biological factor that facilitates the differentiation of chondroprogenitor cells, and 3) a biocompatible and mechanically suitable biomaterial scaffold that resembles the cartilaginous matrix providing a 3D architecture for the cells $[9,48,51]$. Tissue 
engineering requires the use of different cell types, scaffolds, and genetic stimuli to repair or reconstitute articular cartilage. Therefore, we will describe some of the methods utilized in cartilage tissue engineering strategies and the use of low oxygen conditions (e.g. hypoxia) as an enhancer of chondrogenic differentiation.

\subsubsection{Cell sources}

\subsubsection{Chondrocytes}

Cartilage is composed of an abundant extracellular matrix consisting of proteoglycans and collagens type II, IX, and XI which are synthesized by chondrocytes. Brittberg et al. were the first to isolate articular chondrocytes for cartilage repair and developed a technique known as autologous chondrocyte implantation (ACI) [139]. They considered chondrocytes as the ideal cell source for engineering cartilage implants since they retain a chondrogenic phenotype and could synthesize a hyaline articular cartilage extracellular matrix. Although short-term clinical results were good, several disadvantages of the technique were also reported [139]. Among its limitations is the reduced supply of chondrocytes, which requires a long expansion time to obtain sufficient cells for transplantation, increasing the potential to de-differentiate, and promote a fibrocartilage formation instead of a hyaline cartilage repair [65]. Considering the aforementioned problems associated with the use of autologous articular chondrocytes, the control of oxygen tension in cell cultures has become an important parameter for tissue engineering [71]. Several studies have described how in vitro low oxygen cultures are beneficial for the maintenance of a chondrocytic phenotype (Table 2.1). Despite of the culture system, hypoxic culture delays the de-differentiation of murine, bovine and human chondrocytes. Under hypoxia, chondrocytes showed an increase in the synthesis of cartilage extracellular matrix proteins including aggrecan, collagen type II, collagen type IX and collagen type XI [7]. Moreover, low oxygen suppressed hypertrophic differentiation and subsequently terminal differentiation by inhibiting matrix 
degrading enzymes MMP3 and MMP13 [72] and decreasing COL1A1 gene expression [74]. Furthermore, most of the existing literature uses bovine chondrocytes instead of human-isolated chondrocytes. These bovine chondrocytes are obtained from healthy cartilage and have not been exposed to any cartilage degradation. In contrast, the human chondrocytes obtained for the ACI technique, are surrounding by OA synovial fluid and cartilage degrading enzymes. However, how these data can be extrapolated to the human situation remains to be seen.

Previous publications showed an increase in chondrocytes proliferation and cell number under lower oxygen concentrations [75]. Robins et al. also observed that in low oxygen cultures not only COL2A1 was expressed, but also vascular endothelial growth factor (VEGF) was up-regulated [76]. The increase in expression of VEGF correlates with the onset of the healing process found after osteochondral fractures. Hypoxia inducible factor (HIF) regulates VEGF, which participates in angiogenesis and nitric oxide generation, is essential for vasodilation, and causes an increase in blood flow to ischemic tissues [77]. VEGF factors are required during development of collateral vessel growth, blood flow, tissue perfusion, extravasation of inflammatory cells, tissue remodeling and repair [78]. Moreover, local production of VEGF has shown to limit ischemia induced tissue damage and inflammation during chronic hypoxia. VEGFA is a critical mediator of vasculogenesis during development, has neuropotective effects in ischemic brain [79], and has a positive influence on inflammatory response in the lung. Recent studies suggest that VEGFB has a major role in regulating fatty acid and electron transport [80, 81], whereas VEGFC knockout showed embryonically lethality and leads to fluid accumulation due to deficiency of lymphatic drainage $[77,78]$. 


\begin{tabular}{|c|c|c|c|c|c|}
\hline Species & $\begin{array}{l}\text { Oxygen } \\
\text { tension }\end{array}$ & $\begin{array}{l}\text { Culture } \\
\text { Method }\end{array}$ & $\begin{array}{c}\text { Culture } \\
\text { time }\end{array}$ & Results & References \\
\hline \multirow[t]{6}{*}{ Bovine } & $5,21 \%$ & Agarose & 48 hours & †GAGs, Mmps, Adamts5, Mmp13 & 111 \\
\hline & $5,21 \%$ & Alginate beads & 3 weeks & $\begin{array}{l}\uparrow \mathrm{Col} 2 \mathrm{a} 1,[(3) \mathrm{H}] \text { proline and }[(35)] \\
\text { sulfate incorporation }\end{array}$ & 75 \\
\hline & $5,21 \%$ & $\begin{array}{l}\text { Monolayer, } \\
\text { Alginate beads }\end{array}$ & $2-3$ weeks & $\begin{array}{l}\uparrow \text { cell number, Col2a1, Col9a1, } \\
\text { GAGs, proline incorporation }\end{array}$ & 144 \\
\hline & $5,21 \%$ & Alginate beads & 2 weeks & $\begin{array}{l}\uparrow \text { Col2a1, proteoglycan synthesis, } \\
\downarrow \text { Col1a1, proline incorporation }\end{array}$ & 143 \\
\hline & $5,21 \%$ & Alginate beads & 3 weeks & $\begin{array}{c}\uparrow c e l l \text { number, Col2a1, Col10a1, } \\
\text { GAG synthesis }\end{array}$ & 76 \\
\hline & $2,20 \%$ & Alginate beads & 2 weeks & $\begin{array}{c}\uparrow \text { Col2a1, Acan, GAG synthesis, } \\
\downarrow M m p 3, M m p 13\end{array}$ & 72 \\
\hline \multirow{4}{*}{ Human } & $1,21 \%$ & Cell suspension & 16 hours & $\uparrow C O L 1 A 1, H I F 1 A, H I F 2 A, \downarrow C O L 2 A i$ & 11 \\
\hline & $<1,2,5,21 \%$ & Alginate beads & 96 hours & $\downarrow$ Cell viability, $\uparrow$ GAGs & 141 \\
\hline & $2,20 \%$ & $\begin{array}{l}\text { Pellets, PCL } \\
\text { scaffold }\end{array}$ & 4 weeks & $\begin{array}{c}\uparrow C O L 2 A 1, \text { HIST2H4, HIF1A, } \\
\text { SOX9, } \text { ACAN, COL1A1, }\end{array}$ & 74 \\
\hline & $1,20 \%$ & Alginate beads & 48 hours & $\begin{array}{c}\uparrow G D F 1, \text { PHD3, VEGF, SOX9, } \\
\text { COL1A1, MMP13, TGFB2, SOX9, }\end{array}$ & 142 \\
\hline \multirow[t]{2}{*}{ Murine } & $0.5,20 \%$ & Monolayer & 1 week & $\uparrow$ Vegf, Vegf, Hif1a & 5 \\
\hline & $2,21 \%$ & Monolayer & 3 days & $\begin{array}{c}\uparrow A c a n, \text { Col2a1, Sox9, Hif1a, } \\
\downarrow \text { Vegfa, Bmp4, Tgfb }\end{array}$ & 140 \\
\hline
\end{tabular}

\subsubsection{Mesenchymal stem cells}

Mesenchymal stem cells (MSCs) are multipotent progenitor cells that possess the ability to be expanded for up to 40 generations without losing their stemness [73]. They are capable of undergoing differentiation into chondrogenic, osteogenic and adipogenic lineages. These cells are promising candidates for tissue engineering due to the variety of sources (e.g adipose tissue, bone marrow, muscle, periodontal ligament, lung, liver, spleen, thymus, amnion, placenta, umbilical cord blood, synovial membrane and cartilage) and the ease of isolation [12]. The safety of these stem cells has been demonstrated in phase III clincal studies. Currently, 52 clinical studies involving MSCs for osteoarthritis (OA) therapy are referenced at ClinicalTrials.gov [7].

Previous data confirmed that the in vivo implantation of MSCs into cartilage defects induces desirable healing outcomes and improved clinical signs in treated patients $[15,61]$. Riester et al. showed that injection of AMSCs in early stages of OA inhibited synovial thickening and cartilage loss [82]. There is evidence that 
suggests that under more physiological oxygen tension (1-7\% oxygen) MSCs increase their chondrogenic potential, while decreasing hypertrophic differentiation, apoptosis, cellular senescence and endochondral ossification [74]. In hypoxic conditions an elevated glycosaminoglycan production, upregulation of several chondrogenic genes, collagen cross-linking and enhanced mechanical strength has been observed. In vitro experiments have shown HIF1 $\alpha$ is able to potentiate chondrogenesis of MSCs, while inhibiting hypertrophy and subsequently endochondral ossification [40]. In addition, HIF2 $\alpha$ was found to increase the synthesis and cartilaginous matrix production during chondrogenic differentiation of stem cells [83]. Thus, the hypoxia pathway seems crucial for completing chondrogenesis on MSCs.

\subsection{Bone marrow derived stem cells (bMSCs)}

Bone marrow derived mesenchymal stem cells are the most studied and used stem cells for cartilage engineering due to their efficiency in differentiating into the chondrocytic lineage. Among their advantages, the ability to harvest cells from many different bone marrow locations, high proliferation rate, their differentiation capacity and the ability to maintain a relative stable phenotype in vitro endorse them as a good cell source for cartilage repair [84].

In recent years, the use of oxygen levels to control MSC proliferation and differentiation has received major interest. As shown in Table 2.2, several studies have shown that bMSCs cultured under low oxygen tension (1-5\% oxygen) during chondrogenic induction are able to differentiate into hyaline cartilage that better resembles the permanent features of articular cartilage [85]. Human bMSCs treated with TGF $\beta 1,2$ or 3 that were cultured in monolayer and high density pellets under low oxygen conditions, expressed typical articular cartilage biomarkers and inhibitors of hypertrophic differentiation such as COL2A1, SOX9, ACAN, Gremlin 1 (GREM1), Frizzled-related protein (FRZB) and Dickkopf WNT signaling inhibitor 1 (DKK1) [86]. 


\begin{tabular}{|c|c|c|c|c|c|c|c|}
\hline \multicolumn{8}{|c|}{ Table 2.2 bHSCs and low oxygen for cartilage repair strategies } \\
\hline Species & $\begin{array}{l}\text { Oxygen } \\
\text { tension }\end{array}$ & $\begin{array}{l}\text { Cutire } \\
\text { Method }\end{array}$ & $\begin{array}{l}\text { Chondrogenic } \\
\text { Differentiation }\end{array}$ & $\begin{array}{l}\text { Other } \\
\text { Difierentiation }\end{array}$ & $\begin{array}{l}\text { Cufire } \\
\text { time }\end{array}$ & Results & References \\
\hline Bovine & $5.20 \%$ & PCL scaffold & TGFB3 & - & 6 weeks & $\begin{array}{l}\text { †GAGs symthesis, Cofflat, Alpl, } \\
\text { calcium accumulation, Col2a1/Coltat } \\
\text { ratio, Icelularity }\end{array}$ & 155 \\
\hline \multirow[t]{10}{*}{ Human } & $2 \%$ & Monolayer & TGFB3 & $\begin{array}{l}\text { Adipo, Osteo, } \\
\text { Neural, } \\
\text { Endodemal }\end{array}$ & 3 weeks & $\begin{array}{l}\text { †self renewal, cel doubling times, } \\
\text { SOX2, NANOG, OCT4, REST, HIF1A, } \\
\text { RE1, Wht pathway. PHBKJAkt pattway. } \\
\text { |AIF, BAK, p53, B-galactosidase activity }\end{array}$ & 156 \\
\hline & $2,20 \%$ & Monolayer & TGFB3 & $\begin{array}{l}\text { Adipo, Osteo, } \\
\text { Neural, } \\
\text { Endodemal }\end{array}$ & 2 weeks & $\begin{array}{l}\text { tproliferation, OCT4, HIF2A, connexin- } \\
\text { 43. cel density, neural and endodemal } \\
\text { differentiation }\end{array}$ & 157 \\
\hline & $25.21 \%$ & $\begin{array}{l}\text { High-density } \\
\text { peliets }\end{array}$ & TGF $\beta 3$ & - & 5 weeks & $\begin{array}{l}\text { †GAGs synthesis, SOX9, COL2A1, } \\
\text { ACAN, GREM1, FRZB, DKK1, } \\
\text { †COL1OA1, MMP13,PANX3 }\end{array}$ & 85.86 \\
\hline & $\begin{array}{l}<1,21 \% \\
5,21 \%\end{array}$ & $\begin{array}{l}\text { High-density } \\
\text { Monolayer, } \\
\text { High-density } \\
\text { pelets }\end{array}$ & $\begin{array}{l}\text { TGFB1 } \\
\text { TGFB3 }\end{array}$ & $\begin{array}{l}\text { Adipo/Osteogenic } \\
\text { Adipo/Osteogenic }\end{array}$ & $\begin{array}{l}48 \text { hours } \\
4 \text { weeks }\end{array}$ & $\begin{array}{l}\text { †OPN, VEGF, IRUNX2, SP7, COL 1A1 } \\
\text { †colony formation, prolferation, } \\
\text { metabolic activity, osteogenic genes, } \\
\text { alkalne phosphatase activity. } \\
\text { ladioogenesis, chondrogenesis }\end{array}$ & $\begin{array}{l}158 \\
146\end{array}$ \\
\hline & $1.5,21 \%$ & $\begin{array}{l}\text { Monolayer, } \\
\text { Micromass }\end{array}$ & $\begin{array}{l}\text { Chondrocyte } \\
\text { differentiation } \\
\text { media } \\
\text { (Cambrex) }\end{array}$ & Adipo/Osteogenic & 3 weeks & $\begin{array}{l}\text { tcel proliferation, HF1A, COL2A1, not } \\
\text { changes in adipo/osteogenesis }\end{array}$ & 147 \\
\hline & $2,20 \%$ & $\begin{array}{l}\text { Monolayer, } \\
\text { High-density } \\
\text { pelets }\end{array}$ & - & Adipo/Osteogenic & 2 weeks & $\begin{array}{l}\text { tproliferation, cel number, †OCT4, } \\
\text { HIF2A, connexin-43, foronectin }\end{array}$ & 104 \\
\hline & $5,20 \%$ & $\begin{array}{l}\text { High-density } \\
\text { pellets }\end{array}$ & TGFB2 & Hypetrophic & 4 weeks & $\begin{array}{l}\text { tproteoglycan deposition, COL2A1, } \\
\text { \COL 1OA1, VEGF, ALPL, MMP13 }\end{array}$ & 154 \\
\hline & $2,20 \%$ & $\begin{array}{l}\text { Monolayer, } \\
\text { pelet culture }\end{array}$ & TGFB1 & - & 2 weeks & $\begin{array}{l}\text { †sulfated GAGs production, COL2A1, } \\
\text { ACAN, \ADAMTS5, MAP2, MMP13 }\end{array}$ & 40 \\
\hline & $3,21 \%$ & $\begin{array}{l}\text { High-density } \\
\text { peliets }\end{array}$ & TGFB1 & - & 3 weeks & $\begin{array}{l}\text { †colomy formation, GAGs, AGG, } \\
\text { COL1A2, COL2A1, COL1OA1, COMP, } \\
\text { SOX9, HIF1A, HIF2A, TGFB }\end{array}$ & 92 \\
\hline & $2,20 \%$ & $\begin{array}{l}\text { High-density } \\
\text { pelets }\end{array}$ & - & Adipo/Osteogenic & 2 weeks & $\begin{array}{l}\text { †colony fomation, } O C T 4 \text {, fomation of } \\
\text { embryoid bodies, } R E X 1 \text {, ffibronectin }\end{array}$ & 104 \\
\hline Ovine & $5,20 \%$ & $\begin{array}{l}\text { High-density } \\
\text { peliets }\end{array}$ & - & - & 2 weeks & $\begin{array}{l}\text { tpopulation growth, sulphated GAGs, } \\
\text { Col2a1, fosteogenesis }\end{array}$ & 145 \\
\hline Parcine & $5.20 \%$ & $\begin{array}{l}\text { High-density } \\
\text { pelets. } \\
\text { Agarose } \\
\text { hydrogels }\end{array}$ & TGFß3 & Osteogenic & 3 weeks & $\begin{array}{l}\text { †cel colonies, Co/2a1, \colagen } \\
\text { accumulation, alvain red staining. } \\
\text { alkalne phosphatase activity }\end{array}$ & 84 \\
\hline \multirow[t]{2}{*}{ Murine } & $5,20 \%$ & Monolayer & - & Osteogenic & 2 weeks & $\begin{array}{l}\text { †Col2a1, chondroitin sulfate, colony } \\
\text { formation, proliferation rate, osteoblast } \\
\text { differentiation, aka he phosphatase } \\
\text { activity, calcium content, von Kossa } \\
\text { staining. protein synthesis, total } \\
\text { collagen accumulation }\end{array}$ & 159 \\
\hline & $2.20 \%$ & Monolayer & TGFB & - & 3 weeks & $\begin{array}{c}\text { †Col2a1. proteoglycan deposition. } \\
\text { Hifta, Akt, p38 }\end{array}$ & 151 \\
\hline
\end{tabular}

In addition, GAG synthesis and proteoglycan deposition were also increased at lower oxygen levels. The increased expression of hyaline cartilage markers was found after exposing bMSCs to hypoxia for 48 hours and were still present after 5 weeks of culture. In contrast, hypertrophic markers and terminal differentiation decreased under low oxygen tension, as demonstrated by down-regulation of COL10A1, MMP13, RUNX2, SP7, COL1A1, ALPL, reduced Alizarin Red staining and 
alkaline phosphatase activity [87]. Hence, bMSCs are an attractive alternative to chondrocytes for therapeutic approaches in cartilage tissue engineering.

\subsection{Adipose Derived Stem Cells (aMSCs)}

In light of the limitations of bMSCs (e.g. donor site morbidity and relatively low yield of cells), adipose tissue derived MSCs (aMSCs) were studied as an attractive stem cell population to use in cartilage repair. Interestingly, the adipose tissue stromal vascular fraction contains 10 to 100 -fold more clonogenic cells than bone marrow [87], and large quantities can be easily obtained through liposuction. Recent studies indicated that aMSCs are a promising cell source for tissue regeneration and their effects are mostly mediated by their ability to produce various bioactive molecules such as growth factors, cytokines and extracellular vesicles. Despite the large body of evidence indicating the beneficial effects of aMSCs on cartilage regeneration, the efficiency of the therapies are donor specific and change according to patients' age, sex and existent disease [88]. However, low oxygen tension seems to stimulate chondrogenic commitment while inhibiting osteogenic and adipogenic differentiation of aMSCs, as has been reported for bMSCs [73].

Table 2.3 provides a summary of studies that have examined the effect of oxygen tension during chondrogenic differentiation of aMSCs cultured in alginate beads, micromass, high-density pellets, monolayer and PCL scaffolds. Previous results revealed that hypoxia notable enhances chondrogenesis by increasing cell proliferation as well as the expression of chondrogenic markers, ACAN, COL2A1, SOX9, HAPLN1 and COMP. Collectively, published data revealed that low oxygen is an efficient and reliable tool for augmenting chondrogenic differentiation of aMSCs. However, the influence of hypoxia is strongly dependent of experimental settings, culture methods, donor characteristics and oxygen tension. Yet, is still not clear if hypoxia could maintain a stable chondrogenic phenotype of aMSCs while prevent hypertrophic differentiation in vivo, as has 
been published for bMSCs. Future studies could address this issue in adapted animal models for cartilage repair.

\begin{tabular}{|c|c|c|c|c|c|c|c|}
\hline Species & $\begin{array}{l}\text { Oxygen } \\
\text { tension }\end{array}$ & $\begin{array}{l}\text { Culture } \\
\text { Method }\end{array}$ & $\begin{array}{l}\text { Chondrogenic } \\
\text { Differentiation }\end{array}$ & $\begin{array}{c}\text { Other } \\
\text { Differentiation }\end{array}$ & $\begin{array}{c}\text { Culture } \\
\text { time }\end{array}$ & Results & References \\
\hline \multirow[t]{5}{*}{ Human } & $5,20 \%$ & Alginate beads & TGFB1 & - & 2 weeks & $\begin{array}{l}\text { †protein synthesis, total collagen } \\
\text { synthesis, GAGs synthesis, COL2A1, } \\
\text { chondroiti } 4 \text { sulfate, } \downarrow \text { profiferation rate }\end{array}$ & 150 \\
\hline & $2,21 \%$ & $\begin{array}{l}\text { High-density } \\
\text { pelets, } \\
\text { Monolayer }\end{array}$ & TGFB1 & $\begin{array}{l}\text { Adipogenic, } \\
\text { Osteogenic }\end{array}$ & 3 weeks & $\begin{array}{l}\uparrow R E X 1, \text { SOX2, OCT4, NANOG, } \\
\text { HF1A, profferation rate, cel number, } \\
\text { chondrogenesis, \adpogenesis, }\end{array}$ & 105 \\
\hline & $5,21 \%$ & $\begin{array}{l}\text { High-density } \\
\text { pelets }\end{array}$ & TGFB1 & - & 4 weeks & $\begin{array}{c}\uparrow S O X 9, A C \overline{C A N}, \overline{C O L} \bar{A} 1, M M P 13 \\
C O L 1 O A 1\end{array}$ & 122 \\
\hline & $5,20 \%$ & $\begin{array}{l}\text { High-density } \\
\text { pellets }\end{array}$ & TGFB3 & - & 2 weeks & $\begin{array}{l}\text { †HF2A, GAGs synthesis, SOX5, } \\
\text { SOX6, SOX9, ACAN, versican, } \\
\text { COL2A1, COL } 9 A 1, \text { COL11A1, }\end{array}$ & 149 \\
\hline & $2.5,21 \%$ & $\begin{array}{l}\text { High-density } \\
\text { pellets, PCL } \\
\text { scafidd }\end{array}$ & TGFB1 & - & 4 weeks & $\begin{array}{c}\uparrow S O X 9, \text { HAPLN1, COMP, HST2H4, } \\
\text { HIF1A, COL1OA1, GLH, } I H H, \\
\downarrow C O L 1 A 1\end{array}$ & 74 \\
\hline \multirow[t]{3}{*}{ Murine } & $2,21 \%$ & $\begin{array}{l}\text { Micromass, } \\
\text { Mondayer }\end{array}$ & TGFB1 & Osteogenic & 1 week & $\begin{array}{c}\uparrow \text { Cel number, DNA sunthesis, } \\
\text { proliferation, chondrogenesis, } \downarrow \text { Mrp2, } \\
\text { Mrp3, Mrrp8, Mrrp 13, osteogenesis, } \\
\text { Runx2, Opn }\end{array}$ & 106 \\
\hline & $2,21 \%$ & Alginate beads & BMP6 & $\begin{array}{l}\text { Adipogenic, } \\
\text { Osteogenic }\end{array}$ & 2 weeks & $\begin{array}{c}\text { †proliferation rate, Pcna, Col2a1, } \\
\text { adipogenesis }\end{array}$ & 153 \\
\hline & $2,21 \%$ & Micromass & TGFB1 & Osteogenic & 2 weeks & $\begin{array}{c}\text { †prodiferation rate, } \downarrow \text { chondrogenesis, } \\
\text { osteogenesis }\end{array}$ & 152 \\
\hline Rabbit & $5,21 \%$ & Monolayer & TGF $\beta 1$ & - & 4 weeks & $\uparrow A c a n, C o l 2 a 1, \downarrow R L n \times 2$ & 82 \\
\hline
\end{tabular}

\subsection{Other Stem Cells}

Other stem cells sources which are currently reviewed for cartilage tissue engineering are shown in Table 2.4. Synovium derived stem cells (sMSCs) are currently studied as a novel and promising stem cell source for cartilage repair. In physiological conditions sMSCs migrate into articular cartilage lesions where they differentiate into chondrogenic progenitor cells [89]. Several in vitro and animal studies have shown high chondrogenic potential when compared to bMSCs. The advantage of sMSCs cells over other stem cell sources is the ability to grow fast and the less invasive transplantation for the patient. However, their harvesting process is more invasive than for bMSCs. These cells have not been used clinically.

Embryonic stem cells (ESCs) are derived from the inner cell mass of a blastocyst, an early stage pre-implantation embryo. Recent data confirmed that human ESCs 
can be induced in vitro into the chondrogenic lineage using certain microenvironmental conditions [90]. Low oxygen tension has been the microenvironmental condition selected to minimize the spontaneous differentiation of ESCs, while improving their chondrogenic potential even during prolonged cultures (18 months). Despite extensive data looking at the effect of hypoxia effects on ESCs, the effect of low oxygen during chondrogenic differentation of ESCs has not been studied in great detail.

Articular cartilage-derived stem cells (CSCs) are believed to be more committed to the chondrogenic lineage when compared to mesenchymal stem cells that are harvested from outside the joint. Cartilage-derived stem cells are mostly harvested from the superficial zone of adult articular cartilage. In addition, these cells can also be harvested from osteoarthritic (OA) cartilage, which contains larger pools of progenitor cells. CSCs are believed to be primed to differentiate into hyaline cartilage instead of fibrous or hypertrophic tissue making them ideal for cartilage repair [90]. Jayasuriya et al. observed the high chondrogenic potential of these cells, and used them to they successfully repair cartilage defects in 15 patients [91]. In addition, McCarthy et al. found higher expression of COL2A1 in CSCs when compared to articular chondrocytes when implanted in goat chondral defects [129]. These results strongly suggested that cartilage derived progenitor cells have the potential to create hyaline cartilage instead of hypertrophic cartilage once implanted. However, the major limitation of their use in clinical studies is that they account for less than $1 \%$ of all cells found in articular cartilage.

Dermis isolated stem cells (DIAS) are cells isolated from the dermis and have been able to differentiate into the chondrogenic lineage in the presence of cartilage extra cellular matrix. Kaplakci et al. [93] showed that in goat DIAS cultured in micromasses, chondrogenically induced with TGF $\beta 1$ and hypoxia (5\% oxygen) during 2 weeks, the production of type II collagen and 
glycosaminoglycan content was increased. However, low oxygen culture resulted in a significant decrease in cell isolation and proliferation. The use of these cells is, however, promising in the field of tissue engineering due to their availability and negligible damage to the donor site.

\begin{tabular}{|c|c|c|c|c|c|c|c|c|}
\hline \multicolumn{9}{|c|}{ Table 2.4 Other stem cells and low oxygen for cartilage repair strategies } \\
\hline Cell type & Species & $\begin{array}{l}\text { Oxygen } \\
\text { tension }\end{array}$ & $\begin{array}{l}\text { Culture } \\
\text { Method }\end{array}$ & $\begin{array}{l}\text { Chondrogenic } \\
\text { Differentiation }\end{array}$ & $\begin{array}{c}\text { Other } \\
\text { Differentiation }\end{array}$ & $\begin{array}{c}\text { Culture } \\
\text { time }\end{array}$ & Results & References \\
\hline \multirow[t]{3}{*}{ SMSCs } & Human & $2,5,21 \%$ & $\begin{array}{l}\text { Layered } \\
\text { chondrocytes } \\
\text { sheets }\end{array}$ & - & - & 2 weeks & $\begin{array}{c}\text { †cell metabolic activity. } \\
\text { proliferation, proteoglycans, } \\
\text { COL2A1, | MAMP3, MAMP13 }\end{array}$ & 131 \\
\hline & Human & $5.21 \%$ & $\begin{array}{l}\text { High-density } \\
\text { pellets }\end{array}$ & TGFß3, BMP7 & - & 2 weeks & $\begin{array}{l}\text { †colony number, cel number, } \\
\text { proliferation, chondrogenic } \\
\text { potential }\end{array}$ & 88 \\
\hline & Porcine & $5.21 \%$ & $\begin{array}{l}\text { High-density } \\
\text { pellets }\end{array}$ & TGFß3 & - & 2 weeks & $\begin{array}{l}\text { tproliferation, Col2a1, GAGs } \\
\text { synthesis, \Col1a1 }\end{array}$ & 148 \\
\hline \multirow[t]{4}{*}{ ESCs } & Human & $2.21 \%$ & Monolayer & - & - & 3 weeks & $\begin{array}{l}\text { TFGFR1, FGFR2, FRAT2, p- } \\
\text { SMAD2/3, embryoid body } \\
\text { formation, \SOX17, desmin, } \\
\text { GATA4, brachyury, CDX2 }\end{array}$ & 160 \\
\hline & Human & $3,12,21 \%$ & Monolayer & - & - & 3 weeks & $\begin{array}{c}\text { †OCT4NANOG, FOXA2, } \\
\text { SOX17, AFP, GATA4, } \\
\text { Ireduced BrdU incorporation, } \\
\text { aneuploidy }\end{array}$ & 161 \\
\hline & Human & $5.20 \%$ & Monolayer & - & - & 18 months & $\begin{array}{c}\text { †OCT4, NANOG, telomerase } \\
\text { activity }\end{array}$ & 162 \\
\hline & Porcine & $3,5,21 \%$ & Monolayer & - & - & 2 weeks & $\begin{array}{l}\text { tproliferation, formation of } \\
\text { embryoid bodies, Oct4, } \\
\text { embryonic antigen } 4 \text {. Ihuman } \\
\text { chorionic gonadotropin. } \\
\text { progesterone }\end{array}$ & 89 \\
\hline \multirow[t]{2}{*}{ Cscs } & & $2,20 \%$ & Micromass & $\begin{array}{l}\text { BMP4, BMP7. } \\
\text { TGFB1 }\end{array}$ & - & 2 weeks & $\begin{array}{l}\text { tproteoglycan deposition, Szp, } \\
\text { Col2a1, matrix synthesis }\end{array}$ & 90 \\
\hline & Human & $2.20 \%$ & $\begin{array}{l}\text { High-density } \\
\text { pelets }\end{array}$ & TGF $\beta 1$ & - & 2 weeks & $\begin{array}{c}\text { †matrix synthesis, COL } 2 A 1 \\
\lceil\operatorname{COL} 1 A 1, \operatorname{COL} 10 A 1\end{array}$ & 91 \\
\hline DIASs & Goat & $5.20 \%$ & Micromass & TGFß1 & - & 2 weeks & $\begin{array}{c}\text { †Co/2a1, GAGs synthesis, } \\
\text { tproliferation }\end{array}$ & 93 \\
\hline \multirow[t]{2}{*}{ PSCs } & & $1.21 \%$ & Micromass & TGFß1 & $\begin{array}{l}\text { Osteogenic. } \\
\text { Adipogenic }\end{array}$ & 1 week & $\begin{array}{c}\text { †akian bhue stain, Col2a1, } \\
\text { Acan, Vegf, Gut1, Ldja }\end{array}$ & 94 \\
\hline & Rat & $5,20 \%$ & Monolayer & - & Osteogenic & 1 week & $\begin{array}{l}\text { †Hif1a, Vegf, Runx2, Bmp2, } \\
\text { Alp1, Bsp, Ocn. periostin }\end{array}$ & 163 \\
\hline IFPSCs & Porcine & $5,20 \%$ & $\begin{array}{l}\text { Monolayer, } \\
\text { hydrogel }\end{array}$ & TGFß3 & - & 6 weeks & $\begin{array}{l}\text { †ECM production, mechanical } \\
\text { functionalty }\end{array}$ & 50 \\
\hline
\end{tabular}

Another progenitor cell source that is believed suitable for chondroprogenitor cells is the periosteum. Periosteum-derived cells (PSCs) exhibit high self-renewal capacity, stable phenotype throughout expansion and are inducible for chondrogenesis under the correct culture condition. Van Gastel et al. [94] observed that mouse PSCs chondrogenically induced with TGF $\beta 1$ and cultured in a micromass system under hypoxic conditions, significantly increased cartilage formation. These results suggested that under hypoxic conditions PSCs could be 
differentiated into an osteochondroprogenitor cell and be potentially used for osteochondral defects.

Moreover, non-cartilaginous knee joint tissues such as infrapatellar fat pad (IFP) have a significant chondrogenic potential. These tissue could be more available and clinically feasable for collection of chondroprogenitor cells. IFP stem cells cultured in a low oxygen tension of $5 \%$ and in the presence of TGF 33 , enhanced chondrogenesis and improved their mechanical functionality [50].

Despite the increase in the amount of cartilage repair related research in the last decade, the exact mechanism by which stem cells differentiate into permanent hyaline cartilage is still unknown. However, therapeutic approaches that include the use of stem cells in combination with environmental regulation like oxygen tension, have a potential in optimizing chondrogenesis protocols for proper regeneration of articular cartilage. Further investigation including animal models and clinical trials is still required.

\subsubsection{Genetic Manipulation}

There is currently a great need to develop regulatory strategies that can be used in conjunction with stem cells to promote the most desirable repair response. Such strategies include the use of recombinant growth factors, microRNAs or small interfering RNAs (siRNAs) to attenuate further damage. This genetic manipulation could be performed during cell expansion as a strategy to precondition the cells before implantation. In this section, some data will be summarized regarding the use of siRNAs, microRNAs and knockout strategies in combination with hypoxia inducible factors and/or oxygen tension for cartilage repair.

Overexpression of HIF1 $\alpha$ using recombinant adenoviruses in MSCs, enhanced proliferation, migration, cell survival and expression of pro-angiogenic genes. Chromatin immunoprecipitation (ChIP) assays provided evidence for direct 
interaction of HIF $1 \alpha$ with the SOX9 promoter, thus supporting HIF1 $\alpha$ as a key component in the mechanism that regulates chondrogenesis by regulating SOX9 expression [96]. Furthermore, HIF1 $\alpha$ expression can be increased using Cobalt chloride (CoCl2), which inhibits prolylhydroxylases and stimulates HIF1 $\alpha$ breakdown. Even under normoxic conditions, CoCL2 can be used to increase expression of ECM genes and cell viability, and protects chondrocytes from apoptosis in an HIF1 $\alpha$ dependent manner.

In contrast, knockdown and knockout experiments have been performed to study the critical role of hypoxia inducible factor HIF1 $\alpha$ during chondrogenesis and cartilage development. Schipani et al. examined a $H I F 1 \alpha$ deletion using siRNAs in COL2A1 expressing chondrocytes. HIF1 $\alpha$ knockdown accelerated catabolic stress-induced apoptosis and inhibited chondrogenesis [6]. Similarly, mouse articular chondrocytes with an Hif1 $\alpha$ deletion exhibited lower chondrogenic capacity [97]. Thus demonstrating that HIF1 $\alpha$ is critical for the survival of hypoxic chondrocytes and negatively regulates chondrocyte proliferation.

Preliminary data obtained in an conditional knockout model of Hif1 $\alpha$ in limb buds using a Prrx1 promoter, suggested that hypoxia and Hif1 $\alpha$ may play a role in early stages of chondrocyte differentiation [98]. In addition, a dramatic reduction in Sox9 expression followed by differentiation arrest, resulted in severe skeletal malformations. Mice lacking Hif1 $\alpha$ exhibited shorter and deformed bones whereas their growth plates were wider [99]. Chondrocytes in the interior of the developing growth plate displayed increased premature programmed cell death. Furthermore, chondrocytes exposed to hypoxia, but lacking $H I F 1 \alpha$, had markedly decreased expression of COL2A1 and ACAN, suggesting the importance of this pathway in chondrocyte lineage progression [100]. In recent years, HIF2 $\alpha$ participation in chondrogenesis has also been studied. HIF2 $\alpha$ knockdown experiments resulted in a marked decrease in expression of chondrogenic genes 
SOX9 and COL2A1 [83]. Moreover, a conditional KO of Hif2 $\alpha$ in cartilage tissue, inhibited chemokine production and cartilage destruction. Therefore, the effects of hypoxia on chondrogenesis involves both HIF $1 \alpha$ and HIF $2 \alpha$ dependent and independent mechanisms, which rely on cell type and oxygen tension.

Furthermore, hypoxia triggers the expression of microRNAs. MicroRNAs are small non-coding RNA molecules of about 22 nucleotides which principal function is RNA silencing and post-transcriptional regulation of gene expression. Several studies have implicated a large number of microRNAs in regulating preand post-natal chondrogenesis. During the past decade multiple studies have demonstrated that miRNAs are involved in the hypoxic response and contribute to the repression of specific genes under low oxygen conditions [101]. These microRNAs contain HREs in their promoter region and are regulated by HIF transcription factors in response to low oxygen [102]. Although, HIF1 $\alpha$ is known as transcriptional activator, it also functions as transcriptional repressor via induction of miRNAs. Expression of miR-155, miR-424, miR-17-92, miR-21, miR23, miR-24, miR-26, miR-103, miR-107, miR-181 and miR-210 [103-105], were found to be induced under hypoxic conditions and are potential targets of HIF $1 \alpha$. In particular miR-210 was induced during hypoxic preconditioning of stem cells. This causes a cytoprotective effect and helps to maintain cell stemness but also supported cell differentiation [106].

\subsubsection{Mechanical Stimulation}

Because articular cartilage is a tissue subjected to various mechanical stimuli, including hydrostatic pressure and compressive or shear stress, the role of mechanical stimuli in combination with low oxygen tension has been considered to potentiate cartilage repair. Previously it has been described how MSCs are able to sense and discern their mechanical environment [107]. Accordingly, it has been reported that mechanical stimuli can be used for generating cartilageengineered constructs with collagen and glycosaminoglycan content closely 
resembling that of native cartilage [108]. In addition, mechanical stimuli applied to chondrocytes has been found essential for maintaining cartilage integrity. Porcine MSCs embedded in agarose hydrogels supplemented with TGFß3 under low oxygen conditions and dynamic compression reduced GAG accumulation compared to controls. This study concluded that continuous exposure to low oxygen tension was a more potent pro-chondrogenic stimulus than $1 \mathrm{~h} /$ day of dynamic compression. In addition, dynamic compression has been shown to regulate chondrogenesis of bone marrow derived MSCs encapsulated in an agarose gel under low oxygen tension [109]. By preventing neo-vascularization and promoting a local hypoxic environment, mechanical loading could indirectly promote chondrogenesis of MSCs. However, in vitro experiments demonstrated that scaffolds under microenvironmental conditions (low glucose media and low oxygen concentration) with TGFß3 stimulation could also enhance secretion of sulfated glycosaminoglycan and collagen type I [110].

Hydrostatic pressure has shown to promote and increase access to nutrients or from the mechanical stimulation of fluid flow. Elder et al. described how 5\% oxygen in combination with intermittent hydrostatic pressure promoted chondrogenic gene expression in cells [112]. Bovine articular chondrocytes seeded on porous polyurethane scaffolds and cultured under normoxic $\left(21 \% \mathrm{O}_{2}\right)$ or hypoxic $\left(5 \% \mathrm{O}_{2}\right)$ conditions were subjected to a cyclic axial compression (10$20 \%$; $0.5 \mathrm{~Hz}$ ) applied for $1 \mathrm{~h}$ daily using a bioreactor. Cells cultured under reduced oxygen tension resulted in enhanced gene expression of Col2a1, Acan, and higher glycosaminoglycan content, whereas the expression of Col1a1 was down-regulated in standard oxygen cultures $\left(21 \% \mathrm{O}_{2}\right)$. Moreover, histological analysis showed more intense Col2a1 and weaker Col1a1 staining in hypoxic than in normoxic cultures [113]. Therefore, the combination of mechanical stimuli and a hypoxic environment seems to enhance cartilage repair and 
chondrogenesis of cells better than mechanical stimuli alone, by contributing to the stabilization of the chondrocytic phenotype and matrix synthesis.

\subsubsection{Scaffold free cultures and biomaterials for cartilage tissue engineering}

The function of a tissue engineering scaffold is to provide a temporary structure for the cells, which combined with biomechanical or biochemical signals could better fill the defect areas, enhance chondrogenic phenotype of the cells, and promote chondrogenesis on in vivo and/or ex vivo cultures [64]. Because cartilage production varies with scaffold architecture, the importance of choosing the appropriate scaffold for cartilage tissue engineering is vital.

Alternatively, scaffold free culture systems using high density pellets and micromass culture systems are used to stimulate cartilage formation. These culture systems have been proven to increase GAG accumulation under hypoxia conditions [50, 73, 74, 85, 93]. These cellular aggregates place cells in an environment that closely mimics the cell condensation observed during in vivo embryonic development of cartilage [15]. High-density pellets in combination with addition of members of the TGF $\beta$ superfamily were found beneficial for complete in vitro chondrogenesis of MSCs [73]. Ovine MSCs expanded at 5\% $\mathrm{O}_{2}$ revealed a higher proliferation potential and an enhanced chondrogenic differentiation in both pellet culture and in a collagen type I hydrogel compared to those expanded at $20 \% \mathrm{O}_{2}$ [114]. Sheehy et al. demonstrated that MSCs expanded at $5 \% \mathrm{O}_{2}$ proliferated faster resulting in higher cell yields whereas differentiation at $5 \% \quad \mathrm{O}_{2}$ was found to be a more potent promoter of chondrogenesis [84]. Higher COL1A1 and COL10A1 deposition was observed in pellets maintained at normoxia when compared to hypoxia. In a low oxygen environment, pellets demonstrated higher COL2A1 synthesis, a reduced COL10A1 and Alizarin Red staining and alkaline phosphatase activity [84]. Moreover, previous studies showed that implanting co-cultured pellets of stem 
cells and chondrocytes in cartilage knee defects stimulates cartilage formation by increasing ECM synthesis [115].

Despite the good results observed with scaffold-free techniques, tissue engineering also studies the use of scaffolds whose primary objective is to replicate the characteristics of the target-tissue ECM. The scaffold should allow cells to adopt their native morphology and functionality, facilitating the encapsulation of cells and supporting cell growth, proliferation, and differentiation $[116,117]$. Decellularized extra cellular matrices have been used with the idea of preserving the natural architecture of the tissue. Gilpin et al. confirmed that decellularized osteochondral scaffolds can successfully induce primary canine bMSCs to produce repair tissue with a glycosaminoglycan content and stiffness comparable to that of native cartilage under hypoxia $[118,119]$.

Hydrogels are versatile and appealing biomaterials for tissue engineering and cell therapy applications, due to their unique combination of properties similar to natural ECMs, such as mechanical stiffness, elasticity, water content, and degradation [120]. Various types of hydrogels derived from different natural or synthetic polymers have been used for reconstruction of articular cartilage tissues. Lynch et al. observed that after 10 days in culture at $1 \%$ oxygen, collagen production on PVCL-g-HA hydrogels was 10-fold increased compared to meHA controls [121], whereas Portron et al. observed the formation of a cartilaginous tissue when combining preconditioned rabbit or human adipose stromal cells (ASC) in chondrogenic medium containing TGF $\beta$ and cultured within a Si-HPMC hydrogel [122]. Similarly, infrapatellar pad derived stem cells (IFPSCs) differentiated at $5 \%$ oxygen tension and encapsulated in either agarose or fibrin hydrogels exhibited significant GAGs accumulation after 42 days in culture [123]. A low oxygen tension enhanced chondrogenesis of IFPSCs constructs in the presence of TGFB3 [49]. Rat bMSCs cultured on collagen-chitosan microbeads under $5 \% \mathrm{O}_{2}$ (hypoxia), demonstrated that low oxygen enhances initial 
progenitor cell survival. However, chondrogenic differentiation was not strongly supported in any of the microbead formulations [124]. In addition, IFPSCs displayed a diminished chondrogenic potential upon encapsulation in a threedimensional hydrogel compared with pellet culture, synthesizing significantly lower levels of glycosaminoglycan and collagen on a per cell basis [125]. Hence, low oxygen conditions in combination with the appropiate scaffold could help in preprogramming the cells into a chondrogenic lineage while supporting cell proliferation and extra cellular matrix synthesis.

\subsection{CHALLENGES, PROSPECTIVES AND FUTURE DIRECTIONS}

From a surgical perspective, an estimated of 250,000 articular cartilage repair procedures are performed annually in the U.S [126]. However, these cartilage repair therapies do not consistently produce hyaline repair tissue, fill the entirety of the defect or integrate the repair tissue with the adjacent native tissue. The physiological conditions of repeated high-loading biomechanical motion and a low oxygen atmosphere makes the mechanisms of articular cartilage formation and maintenance complex [127]. Given the complexity of articular cartilage in composition, structure, and function, there are still some key challenges to be addressed for cartilage and osteochondral tissue regeneration. Consequently, a succesful cartilage tissue engineering strategy requires the maintainance or increase in COL2A1 matrix deposition while preventing the formation of fibrous cartilage by inhibiting COL1A1 expression and secretion once the cells are implanted in vivo.

Currently, non-genetic approaches are becoming attractive due to their ease of manipulation [28]. Overall, low oxygen tension seems to improve the chondrocytic phenotype of animal and human articular chondrocytes [12] while increasing chondrogenesis on stem cells derived from different sources (e.g fat pad, adipose, bone marrow, cartilage end plate, etc.) as previously described in this chapter $[28,94,128,129]$. Moreover, the combination of low oxygen tension 
(1 to $5 \%$ oxygen) under the adequate biochemical and/or biomechanical induction maintains a chondrocytic phenotype troughout the culture time. Furthermore, the possibility of plating chondrocytic cells on different scaffolds provides an adaptable structure for cell proliferation, differentiation, and enhances ECM synthesis. Altogether, the combination of these approaches could significantly improve the chance for success during in vivo implantation.

Although a large number of studies exhibited an improvement in chondrogenesis, there are still some reports that differ from the positive effects of low oxygen for GAG accumulation and collagen type II synthesis [28, 94, 122, $128,129]$. The variance could be related to differences in culture conditions and the factors used during chondrogenic induction. Moreover, the population characteristics of chondrocytes and stem cells used is also important to achieve good results. However, due to the small number of donors assessed on in vitro studies, conclusions regarding donor to donor variability cannot be stated with confidence.

An adequate balance in low oxygen tension is still required for full cartilage repair. Oxygen tension control will depend on cell population and characteristics and is involved in the delicate balance of stemness, chondrogenic differentiation, and terminal differentiation. Certain hypoxic levels could enhance chondrogenesis of chondroprogenitor cells while a different oxygen level could help increase OA progression. Moreover, prolonged hypoxia could lead to situations in which the intrinsic healing capacity of the tissue stalls. For in vitro cartilage engineering strategies, a delicate balance must be reached between the stimulatory and inhibitory effects of the low supply of oxygen. Further studies are required to elucidate the appropiate oxygen tension for each stage of chondrogenesis and the appropiate time of culture to create in vivo cartilage repair. 
The need to recreate the native cartilage microenvironment at different stages of development will be beneficial to effectively treat the cartilage defect according to the patients needs. Lenas et al. advocate that a methodology to design a tissue in vitro needs to resemble articular cartilage's developmental process and should enable the control of each sequential differentiation step and should block terminal differentiation [130]. Optimization of protocols will indeed be essential to readily generate cartilage implants suitable for clinical use. A preconditioning stage in which the culture conditions mimic the different settings of the native environment, including not only oxygen tension but also mechanical stimulation will be also beneficial. Recent studies used static culture for the preconditioning stage of the cells when using scaffolds. This caused a heterogeneous distribution of the oxygen tension throughout the tissue. Cells in the core of the scaffold will be exposed to lower levels of oxygen to those cells in the outer parts of the scaffold. This heterogeneity could create differences in differentiation, proliferation and ultimately tissue organization [113, 131]. A bioreactor culture is currently under study to allow a more homogeneous tissue formation in vitro and could represent a potential solution to this limitation [132].

Lastly, maintenance of a hypoxia environment in vitro requires large and expensive equipment. Recent findings in this growing field showed crucial influence of hypoxia signalling on chondrocyte maintenance and raised new potential targets to repair cartilage and maintain tissue integrity. Future studies would need to determine an exact mechanism by which low oxygen tension could be firmly maintained during long-term cultures. This thesis focused its efforts in evaluating different molecular mechanisms to mimic a hypoxic environment without the need of a hypoxia chamber and in investigating molecular mechanisms that could support the chondrogenesis generated during low oxygen cultures. 


\section{REFERENCES}

1. Mow V, Bachrach N, Setton L, Guilak F: Stress, Strain, Pressure and Flow Fields in Articular Cartilage and Chondrocytes. Cell Mechanics and Cellular Engineering 1994:345379.

2. Amarilio R, Viukov S, Sharir A, Eshkar-Oren I, Johnson R, Zelzer E: HIF1 $\alpha$ regulation of Sox9 is necessary to maintain differentiation of hypoxic prechondrogenic cells during early skeletogenesis. Development 2007, 134:3917-3928.

3. Brighton C, Heppenstall R: Oxygen Tension in Zones of the Epiphyseal Plate, the Metaphysis and Diaphysis: An in Vitro And In Vitro Stusy In Rats And Rabbits. JBJS 1971, 53:719-728.

4. Silver I: Measurement of $\mathrm{pH}$ and ionic composition of pericellular sites. Philos Trans R Soc Lond B Biol Sci 1975, 271:261-272.

5. Cramer T, Schipani E, Johnson R, Swoboda B, Pfander D: Expression of VEGF isoforms by epiphyseal chondrocytes during low-oxygen tension is HIF-1 alpha dependent. Osteoarthritis Cartilage 2004, 12:433-439.

6. Schipani E, Ryan H, Didrickson S, Kobayashi T, Knight M, Johnson R: Hypoxia in cartilage: HIF-1alpha is essential for chondrocyte growth arrest and survival. Genes Dev 2001, 15:2865-2876.

7. Lund-Olesen K: Oxygen tension in synovial fluids. Arthritis Rheum 1970, 13:769776.

8. Lafont J, Talma S, Hopfgarten C, Murphy C: Hypoxia promotes the differentiated human articular chondrocyte phenotype through SOX9-dependent and independent pathways. J Biol Chem 2008, 283:4778-4786.

9. Mirza E, Pan-Pan C, Wan Ibrahim W, Djordjevic I, Pingguan-Murphy B: Chondroprotective effect of zinc oxide nanoparticles in conjunction with hypoxia on bovine cartilage-matrix synthesis. J Biomed Mater Res A 2015 2015, 103:3554-3563.

10. Hirao M, Tamai N, Tsumaki N, Yoshikawa H, Myoui A: Oxygen tension regulates chondrocyte differentiation and function during endochondral ossification. J Biol Chem 2006, 281:31079-31092.

11. Coimbra I, Jimenez S, Hawkins D, Piera-Velazquez S, Stokes D: Hypoxia inducible factor-1 alpha expression in human normal and osteoarthritic chondrocytes. Osteoarthritis Cartilage 2004, 12:336-345.

12. Ichimaru S, Nakagawa S, Arai Y, Kishida T, Shin-Ya M, Honjo K, Tsuchida S, Inoue H, Fujiwara H, Shimomura S: Hypoxia Potentiates Anabolic Effects of Exogenous Hyaluronic Acid in Rat Articular Cartilage. Int J Mol Sci 2016, 17.

13. Cheng C, Zhang F, Tian J, Tu M, Xiong J, Luo W, Li Y, Song B, Gao S, Lei G: Osteopontin inhibits HIF-2 $\alpha$ mRNA expression in osteoarthritic chondrocytes. Experimental and therapeutic medicine 2015:2415-2419.

14. Mackie E, Ahmed Y, Tatarczuch L, Chen K, Mirams M: Endochondral ossification: how cartilage is converted into bone in the developing skeleton. Int J Biochem Cell Biol 2008, 40:46-62.

15. Pfander D, Cramer T, Schipani E, Johnson R: HIF-1alpha controls extracellular matrix synthesis by epiphyseal chondrocytes. J Cell Sci 2003, 116:1819-1826.

16. Wiesener MS, Turley H, Allen WE, Willam C, Eckardt KU, Talks KL, Wood SM, Gatter KC, Harris AL, Pugh CW: Induction of Endothelial PAS Domain Protein-1 by Hypoxia: Characterization and Comparison With Hypoxia-Inducible Factor-1 $\alpha$. Blood 1998, 92:2260-2268. 
17. Jain S, Maltepe E, Lu MM, Simon C, Bradfield CA: Expression of ARNT, ARNT2, HIF1 $\alpha$, HIF $2 \alpha$ and Ah receptor mRNAs in the developing mouse. Mechanisms of Development 1998, 73:117-123.

18. Gu YZ, Moran SM, Hogenesch JB, Wartman L, Bradfield CA: Molecular characterization and chromosomal localization of a third alpha-class hypoxia inducible factor subunit, HIF3alpha. Gene Expr 1998, 7:205-213.

19. Li Q, Wang X, Yang Y, Lin H: Hypoxia upregulates hypoxia inducible factor (HIF)3alpha expression in lung epithelial cells: characterization and comparison with HIF1alpha. Cell Res 2006, 16:548-558.

20. Wang GL, Jiang BH, Rue EA, Semenza GL: Hypoxia-inducible factor 1 is a basichelix-loop-helix-PAS heterodimer regulated by cellular 02 tension. Proceedings of the National Academy of Sciences 1995, 92:5510-5514.

21. Giaccia A, Simon M, Johnson R: The biology of hypoxia: the role of oxygen sensing in development, normal function, and disease. Genes Dev 2004, 18:2183-2194.

22. Holmquist-Mengelbier L, Fredlund E, Löfstedt T, Noguera R, Navarro S, Nilsson H, Pietras A, Vallon-Christersson J, Borg A, Gradin K : Recruitment of HIF-1alpha and HIF2alpha to common target genes is differentially regulated in neuroblastoma: HIF-2alpha promotes an aggressive phenotype. Cancer Cell 2006, 10:413-423.

23. Raval R, Lau K, Tran M, Sowter H, Mandriota S, Li J, Pugh C, Maxwell P, Harris A, Ratcliffe P: Contrasting properties of hypoxia-inducible factor 1 (HIF-1) and HIF-2 in von Hippel-Lindau-associated renal cell carcinoma. Moll Cell Biol 2005, 25:5675-5686.

24. Krohn K, Link J, Mason R: Molecular Imaging of Hypoxia. Nucl Med 2018, 49:129S-148S.

25. Chen C, Lou T: Hypoxia inducible factors in hepatocellular carcinoma. OncoTarget 2017, 8:46691-46703.

26. Wielockx B, Grinenko T, Mirtschink P, Chavakis T: Hypoxia Pathway Proteins in Normal and Malignant Hematopoiesis Cells 2019, 2:155.

27. Lyer N, Kotch L, Agani F, Leung S, Laughner E, Wenger R, Gassmann M, Gearhart J, Lawler A, Yu A, Semenza G: Cellular and developmental control of 02 homeostasis by hypoxia-inducible factor 1 alpha. Genes Dev 1998, 12:149-162.

28. Coyle $\mathrm{C}$, Izzo $\mathrm{N}$, Chu C: Sustained hypoxia enhances chondrocyte matrix synthesis. J Orthop Res 2009, 27:793-799.

29. Zhong L, Huang X, Karperien M, Post J: The Regulatory Role of Signaling Crosstalk in Hypertrophy of MSCs and Human Articular Chondrocytes. Int J Mol Sci 2015, 16:19225-19247.

30. Fernández-Torres J, Hernández-Díaz C, Espinosa-Morales R, Camacho-Galindo J, Galindo-Sevilla N, López-Macay A, Zamudio-Cuevas Y, Martínez-Flores K, SantamaríaOlmedo G: Polymorphic variation of hypoxia inducible factor-1 A (HIF1A) gene might contribute to the development of knee osteoarthritis: a pilot study. BMC Musculoskelet Disord 2015, 16.

31. Wu WJ, Zhang XK, Zheng XF, Yang YH, Jiang SD, Jiang LS. SHH-dependent knockout of HIF-1 alpha accelerates the degenerative process in mouse. Int J Immunopathol Pharmacol 2013, 26 (3): 601-9.

32. Zuscik M, Hilton M, Zhang X, Chen D, O'Keefe R: Regulation of chondrogenesis and chondrocyte differentiation by stress. J Clin Invest 2008, 118:429-438.

33. Mariani E, Pulsatelli L, Facchini A: Signaling Pathways in Cartilage Repair. Int J Mol Sci 2014, 15:8667-8698. 
34. Hara S, Hamada J, Kobayashi C, Kondo Y, Imura N: Expression and characterization of hypoxia-inducible factor (HIF)-3alpha in human kidney: suppression of HIF-mediated gene expression by HIF-3alpha. Biochem Biophys Res Commun 2001, 287:808-813.

35. Tanaka T, Wiesener M, Bernhardt W, Eckardt K, Warnecke C: The human HIF (hypoxia-inducible factor)-3alpha gene is a HIF-1 target gene and may modulate hypoxic gene induction. . Biochem J 2009, 424:143-151.

36. Li J, Dong S: The Signaling Pathways Involved in Chondrocyte Differentiation and Hypertrophic Differentiation. Stem Cells Int 2016.

37. Araldi E, Khatri R, Giaccia A, Simon M, Schipani E: Lack of HIF-2 $\alpha$ in limb bud mesenchyme causes a modest and transient delay of endochondral bone development. Nat Med 2011, 17:25-26.

38. Hara BP, Urban JP, Maroudas A: Influence of cyclic loading on the nutrition of articular cartilage. Annals of the Rheumatic Diseases 1990, 49:536.

39. Zhou S, Cui Z, Urban JP: Factors influencing the oxygen concentration gradient from the synovial surface of articular cartilage to the cartilage-bone interface: a modeling study. Arthritis Rheum 2004, 50:3915-3924.

40. Markway B, Cho H, Anderson D, Holden P, Ravi V, Little C, Johnstone B: Reoxygenation enhances tumour necrosis factor alpha-induced degradation of the extracellular matrix produced by chondrogenic cells. Eur Cell Mater 2016, 31:425-439.

41. Kean T, Mera H, Whitney G, MacKay D, Awadallah A, Fernandes R, Dennis J: Disparate response of articular- and auricular-derived chondrocytes to oxygen tension. Connect Tissue Res 2016, 57:319-333.

42. Fermor B, Jeffcoat D, Hennerbichler A, Pisetsky DS, Weinberg JB, Guilak F: The effects of cyclic mechanical strain and tumor necrosis factor alpha on the response of cells of the meniscus. Osteoarthritis Cartilage 2004, 12:956-962.

43. Grimshaw MJ, Mason RM: Bovine articular chondrocyte function in vitro depends upon oxygen tension. Osteoarthritis Cartilage 2000, 8:386-392.

44. Kuiper N, Sharma A: A detailed quantitative outcome measure of glycosaminoglycans in human articular cartilage for cell therapy and tissue engineering strategies. Osteoarthritis Cartilage 2015, 23:2233-2241.

45. Hashimoto K, Fukuda K, Yamazaki K, Yamamoto N, Matsushita T, Hayakawa S, Munakata H, Hamanishi C: Hypoxia-induced hyaluronan synthesis by articular chondrocytes: the role of nitric oxide. Inflamm Res 2006, 55:72-77.

46. Masoud G, Li W: HIF-1 $\alpha$ pathway: role, regulation and intervention for cancer therapy. Acta Pharma Sin B 2015, 5:378-389.

47. Kessler M, Grande D: Tissue engineering and cartilage. Organogenesis 2008, 4:28-32.

48. Basson M: Signaling in Cell Differentiation and Morphogenesis. Cold Spring Harb Perspect Biol 2012, 4.

49. Lin G, Hankenson K: Integration of BMP, Wnt, and Notch signaling pathways in osteoblast differentiation. J Cell Biochem 2012, 112:3491-3501.

50. Buckley C, Vinadell T, Kelly D: Oxygen tension differentially regulates the functional properties of cartilaginous tissues engineered from infrapatellar fat pad derived MSCs and articular chondrocytes. Osteoarthritis and Cartilage 2010, 18:13451354. 
51. Dong Y, Drissi H, Chen M, Chen D, Zuscik M, Schwarz E, RJ OK: Wnt-Mediated Regulation of Chondrocyte Maturation: Modulation by TGF- $\beta$. J Cell Biochem 2009, 95:1057-1068.

52. Blom A, Brockbank S, van Lent $P$, van Beuningen $H$, Nozomi J, van der Kraan P: Involvement of the Wnt signaling pathway in experimental and human osteoarthritis: Prominent role of Wnt-induced signaling protein 1. Arthritis \& Rheumatism 2009, 60.

53. Unmarino D: Hypoxia protects against cartilage loss by regulating Wnt signalling. Nature Reviews Rheumatology 2016, 12.

54. Nivison M, Meier K: The role of CCN4/WISP-1 in the cancerous phenotype Cancer Manag Res 2018, 10:2893-2903.

55. Bouaziz W, Sigaux J, Modrowski D, Devignes C, Funck-Brentano T, Richette P, Ea $\mathrm{H}$, Provot S, Cohen-Solal M, Haÿ E: Interaction of HIF1 $\alpha$ and $\beta$-catenin inhibits matrix metalloproteinase 13 expression and prevents cartilage damage in mice. Proc Natl Acad Sci U S A 2016, 113:5453-5458.

56. Ueno M, Maeno T, Nomura M, Aoyagi-Ikeda K, Matsui H, Hara K, Tanaka T, Iso T, Suga T, Kurabayashi M: Hypoxia-inducible factor- $1 \alpha$ mediates TGF- $\beta$-induced PAI-1 production in alveolar macrophages in pulmonary fibrosis. Am J Physiol Lung Cell Mol Physiol 2011, 300:L740-752.

57. Sánchez-Elsner T, Ramírez J, Sanz-Rodriguez F, Varela E, Bernabéu C, Botella L: A cross-talk between hypoxia and TGF-beta orchestrates erythropoietin gene regulation through SP1 and Smads. J Mol Biol 2004, 336:9-24.

58. Haaijman A, Karperien M, Lanske B, Hendriks J, Lowik C, Bronckers A, Burger E: Inhibition of terminal chondrocyte differentiation by bone morphogenetic protein 7 (OP1) in vitro depends on the periarticular region but is independent of parathyroid hormone-related peptide. Bone 1999, 4:397-404.

59. Akkiraju H, Nohe A: Role of Chondrocytes in Cartilage Formation, Progression of Osteoarthritis and Cartilage Regeneration. J Dev Biol 2015, 3:177-192.

60. van Susante J, Buma P, van Osch G, Versleyen D, van der Kraan P, van der Berg W, Homminga G: Culture of chondrocytes in alginate and collagen carrier gels. Acta Orthopaedica Scandinavica 1995, 66:549-556.

61. Treuhaft P, MCCarty D: Synovial fluid pH, lactate, oxygen and carbon dioxide partial pressure in various joint diseases. Arthritis Rheum 1971, 14:475-484.

62. Chung U, Schipani E, McMahon A, Kronenberg H: Indian hedgehog couples chondrogenesis to osteogenesis in endochondral bone development. J Clin Invest 2001, 107:295-304.

63. Bijlsma M, Groot A, Oduro J, Franken R, Schoenmakers S, Peppelenbosch M, Spek C: Hypoxia induces a hedgehog response mediated by HIF-1 $\alpha$. Journal of Cellular and Molecular Medicine 2009, 13.

64. Loeser R: Molecular Mechanisms of Cartilage Destruction: Mechanics, Inflammatory Mediators, and Aging Collide. Arthritis Rheum 2006, 54:1357-1360.

65. Wang G, Zhang Z, Yin H, Bai L, Ma Z, DeCoster M, Wu G: Activation of the sonic hedgehog signaling controls human pulmonary arterial smooth muscle cell proliferation in response to hypoxia. Biochimica et Biophysica Acta (BBA) - Molecular Cell Research 2010, 1803:1359-1367.

66. Chen D, Shen J, Zhao W, Wang T, Han L, Hamilton J, Im H: Osteoarthritis: toward a comprehensive understanding of pathological mechanism. Bone Res 2017, 5:16044. 
67. Kiaer T, Grønlund J, Sørensen K: Subchondral p02, pCO2, pressure, pH, and lactate in human osteoarthritis of the hip. Clin Orthop Relat Res 1988, 229:149-155.

68. Houard X, Goldring M, Berenbaum F: Homeostatic Mechanisms in Articular Cartilage and Role of Inflammation in Osteoarthritis. Curr Rheumatol Rep 2014, 15:375.

69. Loboda A, Jozkowicz A, J D: HIF-1 and HIF-2 transcription factors - Similar but not identical. Molecules and Cells 2010, 29:435-442.

70. Falah M, Nierenberg G, Soudry M, Hayden M, Volpin G: Treatment of articular cartilage lesions of the knee. Int Orthop 2010, 34:621-630.

71. Al-Ani A, Toms D, Kondro D, Thundathil J, Yu Y, Ungrin M: Oxygenation in cell culture: Critical parameters for reproducibility are routinely not reported. PLoS One 2018, 13:e0204269.

72. Heywood H, Nalesso G, Lee D, Dell'Accio F: Culture Expansion in Low-Glucose Conditions Preserves Chondrocyte Differentiation and Enhances Their Subsequent Capacity to Form Cartilage Tissue in Three-Dimensional Culture. BioResearch 2014, 3.

73. Zuliani CC, Andrade KC, Mamoni R, Pereira AH, Coimbra IB.: Micromass cultures are effective for differentiation of human amniotic fluid stem cells into chondrocytes. Clinics 2018.

74. Galeano-Garces C, Riester SM, Dudakovic A, Larson DR, Qu W, Smith J, Dietz AB, Im HJ, Krych AJ, Larson AN, Karperien M, van Wijnen AJ: Molecular Validation of Chondrogenic Differentiation and Hypoxia Responsiveness of Platelet-Lysate Expanded Adipose Tissue-Derived Human Mesenchymal Stromal Cells. Cartilage 2017, 8:283-299.

75. Domm C, Steinhagen J, Freitag S, Kurz B.: Influence of various alginate brands on the redifferentiation of dedifferentiated bovine articular chondrocytes in alginate bead culture under high and low oxygen tension. Tissue Eng 2004, 10:1796-1805.

76. Robins JC, Mukherjee A, Dalal RR, Aronow BJ, Koopman P, Clemens TL: Oxygen tension directs the differentiation pathway of human cytotrophoblast cells. Bone 2005, 37:313-322.

77. Alitalo K, Carmeliet P: Molecular mechanisms of lymphangiogenesis in health and disease. Cancer Cell 2002, 1:219-227.

78. Karkkainen MJ, Haiko P, Sainio K, Partanen J, Taipale J, Petrova TV, Jeltsch M, Jackson DG, Talikka M, Rauvala H, et al: Vascular endothelial growth factor $\mathrm{C}$ is required for sprouting of the first lymphatic vessels from embryonic veins. Nat Immunol 2004, 5:74-80.

79. Sun Y, Jin K, Xie L, Childs J, Mao XO, Logvinova A, Greenberg DA: VEGF-induced neuroprotection, neurogenesis, and angiogenesis after focal cerebral ischemia. J Clin Invest 2003, 111:1843-1851.

80. Hagberg C, Mehlem A, Falkevall A, Muhl L, Eriksson U: Endothelial fatty acid transport: role of vascular endothelial growth factor B. Physiology (Bethesda) 2013, 28:125-134.

81. Hagberg CE, Falkevall A, Wang X, Larsson E, Huusko J, Nilsson I, van Meeteren LA, Samen E, Lu L, Vanwildemeersch M: Vascular endothelial growth factor B controls end othelial fatty acid uptake. Nature 2010, 464:917-921.

82. Riester SM, Lin Y, Jones DL, de Mooij T, Lewallen EA, Nie H, Paradise CR, Radel DJ, Dudakovic A, Camilleri ET, Larson DR, Qu W, Krych AJ, Frick MA, Im HJ, Dietz AB, Smith J, van Wijnen AJ: Safety Studies for Use of Adipose Tissue-Derived Mesenchymal Stromal/Stem Cells in a Rabbit Model for Osteoarthritis to Support a Phase I Clinical Trial. Stem Cells Transl Med 2017, 6:910-922. 
83. Preitschopf A, Kinslechner K, Schütz B, Zwickl H, Rosner M, Gabor JJ, Nehrer S, Hengstschläger M, Mikula M: Rapamycin-Induced Hypoxia Inducible Factor 2A Is Essential for Chondrogenic Differentiation of Amniotic Fluid Stem Cells. Stem Cells Transl Med 2016, 5:580-590.

84. Sheehy EJ, Buckley CT, Kelly DJ: Oxygen tension regulates the osteogenic, chondrogenic and endochondral phenotype of bone marrow derived mesenchymal stem cells. Biochem Biophys Res Commun 2012, 417:305-310.

85. Leijten J, Georgi N, Moreira Teixeira L, van Blitterswijk CA, Post JN, Karperien M: Metabolic programming of mesenchymal stromal cells by oxygen tension directs chondrogenic cell fate. Proc Natl Acad Sci U S A 2014, 111:13954-13959.

86. Zhong L, Dooms E, Leijten J, Verrips T, Khattabi ME, Karperien M, Post JN: Endogenous DKK1 and FRZB Regulate Chondrogenesis and Hypertrophy in ThreeDimensional Cultures of Human Chondrocytes and Human Mesenchymal Stem Cells. Stem Cells Dev 2016, 25:1808-1817.

87. Klar A, Zimoch J, Biedermann T: Skin Tissue Engineering: Application of AdiposeDerived Stem Cells. Biomed Res Int 2017.

88. Ohara T, Nakagawa Y, Matsukura Y, Ichinose S, Koga H, Tsuji K, Sekiya I: Hypoxia enhances proliferation through increase of colony formation rate with chondrogenic potential in primary synovial mesenchymal stem cells. J Med Dent Sci 2016, 63:61-70.

89. Ezashi T, Telugu BP, Roberts RM: Generation of colonies of induced trophoblast cells during standard reprogramming of porcine fibroblasts to induced pluripotent stem cells. Biol Reprod 2011, 85:779-787.

90. Hattori S, Reddi AH: Identification of superficial zone articular chondrocyte stem/progenitor cells. Biochem Biophys Res Commun 2007, 358:99-103.

91. Jayasuriya CT, Newberry J, Desai S, Feltman P, Franco JR, Li N, Terek R, Ehrlich MG, Owens BD: Human Cartilage-Derived Progenitors Resist Terminal Differentiation and Require CXCR4 Activation to Successfully Bridge Meniscus Tissue Tears. Stem cells 2019, 37:102-114.

92. Adesida AB, Mulet-Sierra A, Jomha NM. Hypoxia mediated isolation and expansion enhances the chondrogenic capacity of bone marrow mesenchymal stromal cells. Stem Cell Res Ther. 2012, 3 (2): 9.

93. Kalpakci KN, Hu JC, Athanasiou KA: Cartilage tissue engineering using dermis isolated adult stem cells: the use of hypoxia during expansion versus chondrogenic differentiation. PLoS One 2014, 9:e98570.

94. van Gastel N TS, Roberts SJ, Moermans K, Schrooten J, Carmeliet P, Luttun A, Luyten FP, Carmeliet G: Engineering vascularized bone: osteogenic and proangiogenic potential of murine periosteal cells. Stem Cells 2012, 30:2460-2471.

95. Cheng S, Alarcon C, Mohan S.: Conditional Deletion of the Phd2 Gene in Articular Chondrocytes Accelerates Differentiation and Reduces Articular Cartilage Thickness. Sci Rep 2017, 7:45408.

96. Provot S, Gunes Y, Kathri R, Le Q, Kronenberg HM, Johnson RS, Longaker MT, Giaccia AJ, Schipani E.: Hif-1alpha regulates differentiation of limb bud mesenchyme and joint development. J Cell Biol 2007, 177:451-464.

97. Aro E, Gerard-O'Riley R, Mangiavini L, Myllyharju J, Schipani E: Hypoxiainducible Factor-1 (HIF-1) but Not HIF-2 Is Essential for Hypoxic Induction of Collagen Prolyl 4-Hydroxylases in Primary Newborn Mouse Epiphyseal Growth Plate Chondrocytes. J Biol Chem 2012, 287:37134-37144. 
98. Mircea I, Huang X: miR-210: Fine-Tuning the Hypoxic Response. Adv Exp Med biol 2015, 772:205-227.

99. Kulshreshtha R, Ferracin M, Wojcik SE, Garzon R, Alder H, Agosto-Perez FJ, Davuluri R, Liu CG, Croce CM, Negrini M: A microRNA signature of hypoxia. Mol Cell Biol 2007, 27:1859-1867.

100. Huang F, Zhu X, Hu XQ, Fang ZF, Tang L, Lu XL, Zhou SH: Mesenchymal stem cells modified with miR-126 release angiogenic factors and activate Notch ligand Delta-like-4, enhancing ischemic angiogenesis and cell survival. Int J Mol Med 2013, 31:484-492.

101. Sarkar S, Dey BK, Dutta A: MiR-322/424 and -503 are induced during muscle differentiation and promote cell cycle quiescence and differentiation by down-regulation of Cdc25A. Mol Biol Cell 2010, 21:2138-2149.

102. Chen W, Cai F, Zhang B, Barekati Z, Zhong XY: The level of circulating miRNA-10b and miRNA-373 in detecting lymph node metastasis of breast cancer: potential biomarkers. Tumour Biol 2013, 34:455-462.

103. Pei M: Environmental preconditioning rejuvenates adult stem cells' proliferation and chondrogenic potential. Biomaterials 2017, 117:10-23.

104. Grayson WL, Zhao F, Bunnell B, Ma T. Hypoxia enhances proliferation and tissue formation of human mesenchymal stem cells. Biochem Biophys Res Commun. 2007 Jul 6. 358 (3): 948-53.

105. Choi JR, Pingguan-Murphy B, Wan Abas WA, Noor Azmi MA, Omar SZ, Chua KH, Wan Safwani WK. Impact of low oxygen tension on stemnes, proliferation and differentiation potential of human adipose-derived stem cells. Biochem Biophys Res Commun. 2014, 448: 210-224.

106. Xu Y, Malladi P, Chiou M, Bekerman E, Giaccia AJ, Longaker MT. In vitro expansion of adipose-derived adult stromal cells in hypoxia enhances early chondrogenesis. Tissue Eng. 2007. 13: 2981-2993.

107. Kelly DJ, Prendergast PJ: Mechano-regulation of stem cell differentiation and tissue regeneration in osteochondral defects. J Biomech 2005, 38:1413-1422.

108. Lijie Zhang JH, Kyriacos A. Athanasiou: The Role of Tissue Engineering in Articular Cartilage Repair and Regeneration. Crit Rev Biomed Eng 2011, 37:1-57.

109. Kim H, Bae C, Kook YM, Koh WG, Lee K, Park MH: Mesenchymal stem cell 3D encapsulation technologies for biomimetic microenvironment in tissue regeneration. Stem Cell Res Ther 2019, 10:51.

110. Guillaume O, Daly A, Lennon K, Gansau J, Buckley SF, Buckley CT: Shape-memory porous alginate scaffolds for regeneration of the annulus fibrosus: effect of TGF-beta3 supplementation and oxygen culture conditions. Acta Biomater 2014, 10:1985-1995.

111. Tilwani RK, Vessillier S, Pingguan-Murphy B, Lee DA, Bader DL, Chowdhury TT: Oxygen tension modulates the effects of TNFalpha in compressed chondrocytes. Inflamm Res 2017, 66:49-58.

112. Elder B, Athanasiou K: Hydrostatic Pressure in Articular Cartilage Tissue Engineering: From Chondrocytes to Tissue Regeneration. Rissue Eng Part B 2009, 15:4353.

113. Wernike E, Li Z, Alini M, Grad S: Effect of reduced oxygen tension and long-term mechanical stimulation on chondrocyte-polymer constructs. Cell Tissue Res 2008, 331:473-483. 
114. Zscharnack M, Poesel C, Galle J, Bader A: Low oxygen expansion improves subsequent chondrogenesis of ovine bone-marrow-derived mesenchymal stem cells in collagen type I hydrogel. Cells Tissues Organs 2009, 190:81-93.

115. Wongin SW, Chotiyarnwong P, Siriwatwechakul W, Kino-oka M, Kim MH, Viravaidya-Pasuwat K: Maintenance of human chondrogenic phenotype on a dendrimerimmobilized surface for an application of cell sheet engineering. BMC Biotechnol 2018, 18.

116. Wang B, Chen X, Zeng C, Hu Q, Yin W, Li W, Xie H, Zhang B, Huang X, Yu F: Nanoparticle-modified chitosan-agarose-gelatin scaffold for sustained release of SDF-1 and BMP-2. Int J Nanomedicine 2018, 13:7395-7408.

117. Iturriaga L, Hernaez-Moya R, Erezuma I, Dolatshahi-Pirouz A, Orive G: Advances in stem cell therapy for cartilage regeneration in osteoarthritis. Expert Opin Biol Ther 2018, 18:883-896.

118. Gilpin A, Yang Y: Decellularization Strategies for Regenerative Medicine: From Processing Techniques to Applications. Biomed Res Int 2017:9831534.

119. Su J, Chen S, Chen Y, Chen W: Evaluation of Magnetic Nanoparticle-Labeled Chondrocytes Cultivated on a Type II Collagen-Chitosan/Poly(Lactic-co-Glycolic) Acid Biphasic Scaffold. Int J Mol Sci 2017, 17:87.

120. Izadifar Z, Chen X, Kulyk W: Strategic Design and Fabrication of Engineered Scaffolds for Articular Cartilage Repair. J Funct Biomater 2012, 3:799-838.

121. Lynch B, Crawford K, Baruti O, Abdulahad A, Webster M: The effect of hypoxia on thermosensitive poly(N-vinylcaprolactam) hydrogels with tunable mechanical integrity for cartilage tissue engineering. J Biomed Mater Res B Appl Biomater 2017, 105:18631873.

122. Portron S, Merceron C, Gauthier O, Lesoeur J, Sourice S, Masson M, Fellah BH, Geffroy 0, Lallemand E, Weiss P: Effects of in vitro low oxygen tension preconditioning of adipose stromal cells on their in vivo chondrogenic potential: application in cartilage tissue repair. PLoS One 2013, 8:e62368.

123. O'HEireamhoin S, Buckley CT, Jones E, McGonagle D, Mulhall KJ, Kelly DJ: Recapitulating aspects of the oxygen and substrate environment of the damaged joint milieu for stem cell-based cartilage tissue engineering. Tissue Eng Part C Methods 2013, 19:117-127.

124. Wise JK, Alford AI, Goldstein SA, Stegemann JP: Comparison of uncultured marrow mononuclear cells and culture-expanded mesenchymal stem cells in 3D collagen-chitosan microbeads for orthopedic tissue engineering. Tissue Eng Part A 2014, 20:210-224.

125. Lu CW, Hu YY, Bai JP, Liu J, Meng GL, Lu R: [Three dimensional induction of autologous mesenchymal stem cell and the effects on depressing long-term degeneration of tissue-engineering cartilage]. Zhonghua Wai Ke Za Zhi 2007, 45:1717-1721.

126. Grande D, Schwartz J, Brandel E, Chahine N, Sgaglione N: Articular Cartilage Repair Where We Have Been, Where We Are Now, and Where We Are Headed. Cartilage 2013, 4:281-285.

127. Milner P, Wilkins R, Gibson J: Cellular Physiology of Articular Cartilage in Health and Disease. Cambridge, UK: InTech; 2012.

128. Georgi N, Eijkel GB, Periyasamy PC, Kiss A, van Blitterswijk C, Post JN, Heeren RM, Karperien M.: Differentiation of mesenchymal stem cells under hypoxia and 
normoxia: lipid profiles revealed by time-of-flight secondary ion mass spectrometry and multivariate analysis. Anal Chem 2015, 87:3981-3988.

129. McCarthy HE, Bara JJ, Brakspear K, Singhrao SK, Archer CW. The comparison of equine articular cartilage progenitor cells and bone marrow derived stromal cells as potential cell sources for cartilage repair in the horse. Vet J. 202 Jun. 192 (3): 345-51.

130. Lenas P, Ikonomou L: Developmental engineering: design of clinically efficacious bioartificial tissues through developmental and systems biology. Sci China Life Sci 2018, 61:978-981.

131. Neybecker P, Henrionnet C, Pape E, Mainard D, Galois L, Loeuille D, Gillet P, Pinzano A. In vitro and in vivo potentialities for cartilage repair from human advanced knee osteoarthritis synovial fluid derived mesenchymal stem cells. Stem Cell Res Ther. 2018 Nov. 9 (1): 329.

132. Kang H, Lu S, Peng J, Yang Q, Liu S, Zhang L, Huang J, Sui X, Zhao B, Wang A: In vivo construction of tissue-engineered cartilage using adipose-derived stem cells and bioreactor technology. Cell Tissue Bank 2015, 16:123-133.

133. Leijten J, Emons J, Sticht C, van Gool S, Decker E, Uitterlinden A, Rappold G, Hofman A, Rivadeneira F, Scherjon S, Wit JM, van Meurs J, van Blitterswijk CA, Karperien M. Gremlin, frizzled-related protein, and Dkk-1 are key regulators of human articular cartilafe homeostasis. Arthritis Rheum. 2012.64 (10): 3302-12.

134. Cleary MA, Narcisi R, Albiero A, Jenner F, de Kroon LMG, Koevoet WJLM, Brama PAJ, van Osch GJVM. Dynamic regulation of TWIST1 expression during chondrogenic differentiation of human bone marrow-derived mesenchymal stem cells. Stem Cells Dev. 2017. 26 (10): 751-761.

135. Green JD, Tollemar V, Dougherty M, Yan Z, Yin L, Ye J, Collier Z, Mohammed MK, Haydon RC, Luu HH, Kang R, Lee MJ, Ho SH, He TC, Shi LL, Athiviraham A. Multifaceted signaling regulators of chondrogenesis: Implications in cartilage regeneration and tissue engineering. Genes Dis. 2015. 2 (4): 307-327.

136. Steinert AF, Weissenberger M, Kunz M, Gilbert F, Ghivizzani SC, Gobel S, Jakob F, Noth $\mathrm{U}$, Rudert $\mathrm{M}$. Indian hedgehog gene transfer is a chondrogenic inducer of human mesenchymal stem cells. Arthritis Res Ther. 2012. 14 (4): R168.

137. Suominen MY. Rheumatoid arthritis revisited. Med Hypotheses. 2019. 122:56.

138. Nguyen CT, Bloch Y, Skladananowska K, Savvides SN, Adamopolous IE. Patophysiology and inhibition of IL23 signaling in psoriatic arthritis: A molecular insight. Clin Immunol. 2018 Sept. S1521-6616 (18) 30476-5.

139. Brittberg M. Autologous chondrocyte implantation-technique and long term follow up. Injury. 2008. 39 Suppl 1: S40-9.

140. Shi S, Xie J, Zhong J, Lin S, Zhang T, Sun K, Fu N, Shao X, Lin Y. Effects of low oxygen tension on gene profile of soluble growth factors in co-cultured adipose-derived stromal cells and chondrocytes. Cell proliferation. 49 (3): 341-351.

141. Collins JA, Moots RJ, Winstanley R, Clegg PD, Milner PI. Oxygen and pH- sensitivity of human osteoarthritic chondrocytes in 3-D alginate bead culture system. Osteoarthritis Cartilage. 2013 Nov. 21 (11): 1790-1798.

142. Das R, Timur UT, Edip S, Haak E, Wruck C, Weinas H, Jahr H. TGFB2 is involved in the preservation of the chondrocyte phenotype under hypoxic conditions . Ann Anat. 2015 Mar, 198:1-10. 
143. Kurz B, Domm C, Jin M, Sellckau R, Shunke M. Tissue engineering of articular cartilafe under the influence of collagen I/III membranes and low oxygen tension. Tissue Eng. 2004 Jul. 10 (7-8): 1277-86.

144. Domm C, Shunke M, Christesen K, Kurz B. Redifferentiation of dedifferentiated bovine articular chondrocytes in alginate culture under low oxygen tension. Osteoarthritis Cartilage. 2002 Jan. 10 (1): 13-22.

145. Krinner A, Zscharnack M, Bader A, Drasdo D, Galle J. Impact of oxygen environment on mesenchymal stem cell expansion and chondrogenic differentiation. Cell Prolif. 2009. 42: 471-484.

146. Boyette LB, Creasey OA, Guzik L, Lozito T, Tuan RS. Human bone marrow-derived mesenchymal stem cells display enhanced clonogenicity but impaired differentiation with hypoxic preconditioning. Stem Cells Transl Med. 2014;3:241-254.

147. Martin-Rendon E, Hale SJ, Ryan D, Baban D, Forde SP, Roubelakis M, Sweeney D, Moukayed M, Harris AL, Davies K, Watt SM. Transcriptional profiling of human cord blood CD133+ and cultured bone marrow mesenchymal stem cells in response to hypoxia. Stem Cells. 2007;25:1003-1012.

148. Li J, Pei M. Optimization of an in vitro three-dimensional microenvironment to reprogram synovium-derived stem cells for cartilage tissue engineering. Tissue Eng Part A. $2011 ; 17: 703-712$.

149. Khan WS, Adesida AB, Hardingham TE. Hypoxic conditions increase hypoxiainducible transcription factor 2 alpha and enhance chondrogenesis in stem cells from the infrapatellar fat pad of osteoarthritis patients. Arthritis Res Ther. 2007;9:R55.

150. Wang DW, Fermor B, Gimble JM, Awad HA, Guilak F. Influence of oxygen on the proliferation and metabolism of adipose derived adult stem cells.J Cell Physiol. 2005;204:184-191.

151. Kanichai M, Ferguson D, Prendergast PJ, Campbell VA. Hypoxia promotes chondrogenesis in rat mesenchymal stem cells: a role for AKT and hypoxia-inducible factor (HIF)-1alpha. J Cell Physiol. 2008;216:708-715.

152. Malladi P, Xu Y, Chiou M, Giaccia AJ, Longaker MT. Effect of reduced oxygen tension on chondrogenesis and osteogenesis in adipose-derived mesenchymal cells. Am J Physiol Cell Physiol. 2006;290:C1139-C1146.

153. Malladi P, Xu Y, Chiou M, Giaccia AJ, Longaker MT. Hypoxia inducible factor-1alpha deficiency affects chondrogenesis of adipose-derived adult stromal cells. Tissue Eng. 2007;13:1159-1171.

154. Gawlitta D, van Rijen MHP, Schrijver EJM, Alblas J, Dhert WJA. Hypoxia impedes hypertrophic chondrogenesis of human multipotent stromal cells. Tissue Eng Part A. 2012; 18:1957-66.

155. Meretoja VV, Dahlin RL, Wright S, Kasper FK, Mikos AG. The effect of hypoxia on the chondrogenic differentiation of co-cultured articular chondrocytes and mesenchymal stem cells in scaffolds. Biomaterials. 2013 Jun. 34 (17): 4266-4273.

156. Rios C, D’Ippolito G, Curtis KM, Delcroix GJR, Gomez LA, El Hokayem J, Rieger M, Parrondo R, de las Pozas A, Perez-Stable C, Howard GA, Schiller PC. Low oxygen modulates multiple signaling pathways, increasing self renewal, while decreasing differentiation, senescence, and apoptosis in stromal MIAMI cells. Stem Cells Dev. 2016 Jun. 25 (11): 848-860. 
157. D'Ippolito G, Schiller PC, Ricordi C, Roos BA, Howard GA. Age-related osteogenic potential of mesenchymal stromal stem cells from human vertebral bone marrow. J Bone Res. 1999 Jul. 14 (7): 1115-22.

158. Potier E, Ferreira E, Andriamanalijaona R, Pujol JP, Oudina K, Logeart-Avramoglou D, Petite H. Hypoxia affects mesenchymal stromal cells osteogenic differentiation and angiogenic factor expression. Bone. 2007 Apr. 40 (4): 1078-87.

159. Lennon DP, Edmison JM, Caplan AI. Cultivation of rat marrow derived mesenchymal stem cells in reduced oxygen tension: effects on in vitro and in vivo osteochondrogenesis. J Cell Physiol. 2001 Jun. 187 (3): 345-55.

160. Chen HF, Kuo HC, Lin SP, Chien CL, Chiang MS, Ho HN. Hypoxic culture maintains self-renewal and enhances embryoid body formation of human embryonic stem cells. Tissue Eng Part A. 2010 Sep. 16 (9): 2901-13.

161. Lim HJ, Han J, Woo DH, Kim SE, Kim SK, Kang HG, Kim JH. Biochemical and morphological effects of hypoxic environment on human embryonic stem cells in longterm culture and differentiating embryoid bodies. Mol Cells. 2011 Feb;31(2):123-32.

162. Zachar V, Prasad SM, Weli SC, Gabrielsen A, Petersen K, Petersen MB, Fink T. The effect of human embryonic stem cells (hESCs) long-term normoxic and hypoxic cultures on the maintenance of pluripotency. In Vitro Cell Dev Biol Anim. 2010 Apr;46(3-4):27683.

163. Mendes LF, Katagiri H, Tam WL, Chai YC, Geris L, Roberts SJ, Luyten FP. Advancing osteochondral tissue engineering: bone morphogenetic protein, transforming growth factor, and fibroblast growth factor signaling drive ordered differentiation of periosteal cells resulting in stable cartilage and bone formation in vivo. Stem Cell Res Ther. 2018 Feb 21;9(1):42. 


\section{CHAPTER 3}

3. Hypoxia promotes in vitro chondrogenic differentiation of adipose derived stem cells (aMSCs) in Three-Dimensional cultures. 


\section{ABSTRACT}

To optimize human adipose-derived mesenchymal stem cells (aMSCs) and primary chondrocytes for therapeutic applications in cartilage regeneration, we investigated the role of low oxygen culture on chondrogenic differentiation. Cells were cultured for 21 days in chondrogenic media under normoxic (20\% oxygen) or hypoxic (2\% oxygen) conditions using two distinct 3-dimensional (3D) culture methods (high-density pellets and cells seeded in poly-ع-caprolactone scaffolds). Gene expression of chondrocyte related genes and histological analysis was monitored during differentiation. The expression of a panel of cartilage markers including COL2A1, ACAN and DCN, was up regulated during chondrogenic differentiation in both 3D-culture systems and both aMSCs and human chondrocytes. Expression of several cartilage-related genes was modulated independent of oxygen levels, but we observed cell type-specific responses that depend on low oxygen or the type of 3D-culture system. For example, expression of ACAN and HAPLN1 was more robust in pellet cultures when compared to scaffold cultures and hypoxia enhanced the expression of chondrogenic genes in scaffold cultures. Histological analysis revealed that both 3D-culture methods support formation of a glycosaminoglycan and COL2A1 positive matrix.

Cell type specific effects of low oxygen and 3D environments indicate that mesenchymal cell fate and differentiation potential is remarkably sensitive to oxygen. Genetic programming of aMSCs to a chondrocytic phenotype is effective under hypoxic conditions as evidenced by increased expression of cartilagerelated biomarkers and biosynthesis of a glycosaminoglycan positive matrix. Furthermore, under low oxygen conditions 3D-culture environments improve the chondrogenic potential of aMSCs even further. 


\subsection{INTRODUCTION}

Articular cartilage is a well-organized tissue that is adapted for friction-free synovial joint movements. Focal cartilage defects in articular cartilage caused by trauma or osteochondritis dissecans are leading causes of osteoarthritis (OA) in young patients [1]. When these focal lesions are loaded, increased stress on the surrounding cartilage leads to expansion of the defect size and further cartilage breakdown. Articular cartilage has reduced healing capacity that has been attributed to avascularity, reduced cellularity and low cell turnover [2]. In particular, the poor vascularity may impair normal tissue repair by humoral factors and stem/progenitor cells [3]. Due to the limited healing capacity, the regeneration of articular cartilage surface is critical in order to prevent future progression of degenerative arthritis and $\mathrm{OA}$.

Treatments for symptomatic relief using injections of cortisone and hyaluronic acid, and cartilage repair stimulation procedures such as microfacture, subchondral drilling, abrasion, arthroplasty, and osteochondral graft transfers, which attempt to restore normal joint kinematics, have been performed for many years with some clinical success [4]. Currently, cell-based therapies such as autologous chondrocyte implantation (ACI) have become the gold standard for cartilage repair and regeneration. ACI is widely used for functional restoration of focal injuries $\left(2-4 \mathrm{~cm}^{2}\right)[5,6]$ and has demonstrated promising clinical results in younger patients [7]. However, repair of more diffuse articular cartilage lesions is poor, especially in diffuse osteoarthritis in older patients. The ACI procedure has a number of limitations [8-10], including donor site morbidity, limited source of cells, limited expansion in vitro and production of fibrocartilage. In addition, the major drawback of ACI is the dedifferentiation of chondrocytes resulting from the expansion of the chondrocytes for multiple passages in monolayer in order to acquire a workable amount of cells for downstream implantation [11]. Furthermore, this dedifferentiation is defined by the gradual loss of chondrocyte phenotype. 
As dedifferentiation progresses, molecular markers associated with chondrocytes and cartilage formation, such as aggrecan (ACAN), type II collagen (COL2A1), decorin (DCN), cartilage oligomeric matrix protein (COMP) and SRY (sex determining region Y)-box 9 (SOX9), start to decrease, whereas the expression of fibroblastic and osteogenic markers increase [12]. Moreover, spontaneous articular cartilage healing typically occurs via the development of fibrocartilaginous scar tissue that exhibits inferior biomechanical properties to hyaline articular cartilage and which may interfere with currently available clinical strategies for cartilage repair [13]. Fibrocartilaginous healing also fails to restore friction-free motion and is unable to withstand normal loads and compression forces, thus placing the joint at risk for progressive degeneration [14]. Promoting effective articular cartilage regeneration remains a clinical challenge.

Use of stem cells, and in particular mesenchymal stem cells (MSCs) from adult sources, has emerged as a viable solution to overcome limitations of current cartilage restoration procedures. Multipotent adult MSCs reside in several tissues including bone marrow, skeletal muscle, neural tissue, adipose tissue and synovium. MSCs are currently being used in various clinical trials with promising clinical results $[15,16]$. However, promoting chondrogenic differentiation of MSCs can be challenging. Currently, most studies for cartilage repair have focused on bone marrow derived MSCs. As an alternative, adipose-tissue derived human mesenchymal stem cells (aMSCs) are an attractive cellular therapeutic due to minimally invasive tissue harvest, high abundance of cells and rapid expansion ex vivo [17]. Moreover, these cells are multipotent and can produce musculoskeletal extracellular matrix proteins once confluent and are able to differentiate into osteogenic and chondrogenic lineages $[18,19]$.

While there are many studies on chondrogenic differentiation of bone marrow derived MSCs, our studies address a major gap in our knowledge by investigating the chondrogenic potential of aMSCs under multiple culture conditions relevant 
to cartilage tissue engineering, including hypoxia and propagation on 3D culture environment. The function of a tissue engineering scaffold to provide a temporary structure for the cells is evaluated [19]. Electrospun polymeric nanofibers emerged as potential scaffold for cartilage repair due to their capacity of mimicking the natural extracellular matrix $[19,33]$. These scaffolds combined with the appropiate biochemical signals could better fill the defect areas, enhance chondrogenic phenotype of the cells, and promote chondrogenesis on in vivo and/or ex vivo cultures [33]. Moreover, a side-by-side comparison between aMSC and primary chondrocyte gene expression and histological analysis provides a new insight in the field.

\subsection{MATERIALS AND METHODS}

\subsubsection{Cell harvest and monolayer culture.}

Human adipose tissue derived mesenchymal stromal cells (aMSCs) were derived from lipo-aspirates obtained from consenting healthy donors. Human primary chondrocytes were obtained from the calcaneal apophysis of consenting donors. Studies with both cell types were approved by the Mayo Clinic Institutional Review Board (IRB). Maintenance media for aMSCs was comprised of advanced MEM (Gibco/Thermo Fisher Scientific, Waltham, MA) supplemented with 5\% human platelet lysate (PL-Max, MillCreek Life Sciences, Rochester, MN), 1\% penicillin/streptomycin, 1\% Glutamax (Gibco/Thermo Fisher Scientific) and $0.2 \%$ Heparin (Baxter, Deerfield, IL). Human primary chondrocytes were cultured in maintenance medium that contained advanced MEM (Gibco/Thermo Fisher Scientific) supplemented with $10 \%$ fetal bovine serum (Atlanta Biologicals, Atlanta, GA, Atlanta, GA) and 1\% penicillin/streptomycin (Gibco/Thermo Fisher Scientific). Cells were cultured using standard procedures in $\mathrm{T}-175 \mathrm{~cm}^{2}$ flasks at $37{ }^{\circ} \mathrm{C}, 95 \%$ humidity and $5 \% \mathrm{CO}_{2}$ until they reached $80 \%$ confluence. 
Prior to each experiment, cells were detached from T175 flasks by trypsinization using TrypLE Express (Gibco/Thermo Fisher Scientific). For monolayer culture, aMSCs were plated in 6 well-plates at a density of 3,000 cells $/ \mathrm{cm}^{2}$ and maintained for 21 days in chondrogenic media which consisted of serum- or platelet lysate-free culture media supplemented with $40 \mathrm{mg} / \mathrm{ml}$ of L-proline (Sigma-Aldrich, Saint-Louis, MO), $50 \mathrm{mg} / \mathrm{ml}$ Insulin Transferrin Selenium-premix (Gibco/Thermo Fisher Scientific), $50 \mathrm{mg} / \mathrm{ml}$ of ascorbic acid (Sigma-Aldrich), 10 $\mathrm{ng} / \mathrm{ml}$ of TGF- $\beta 1$ and $0.1 \mu \mathrm{M}$ dexamethasone (Sigma-Aldrich). Cell culture medium was replaced every three days.

\subsubsection{Fabrication of nanofibrous poly- $\varepsilon$-caprolactone (PCL) scaffolds}

Scaffolds were fabricated using an electrospinning technique described previously [20]. Scaffolds were composed of $9.5 \mathrm{wt} \%$ homogeneous solution of

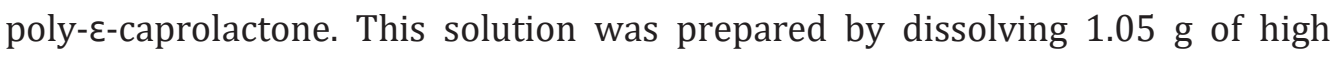
molecular weigth $(80,000)$ poly- $\varepsilon$-caprolactone (Sigma-Aldrich) in $0.8 \mathrm{~g} \mathrm{~N}, \mathrm{~N}$ dimethylformamide (Thermo Fisher Scientific), 6.0 chloroform (Thermo Fisher Scientific) and $3.2 \mathrm{~mL}$ acetone (Thermo Fisher Scientific). The solution was stirred for 4 hours. The polymer solution was subsequently placed in a $20 \mathrm{ml}$ glass syringe with a $20-\mathrm{G}$ needle. The solution was dispensed at a rate of $3.3 \mathrm{ml} / \mathrm{h}$ while applying a $30 \mathrm{kv}$ electrical field. All procedures were performed in a chemical hood. A glass plate mounted on an aluminum block placed $15 \mathrm{~cm}$ away from the needle collected the fibers. This procedure was followed until the sheets reached a thickness of $1 \mathrm{~mm}$. Discs of $10 \mathrm{~mm}$ in diameter were cut from the sheets using a dermal punch. Both sides of the scaffold were sterilized by ultraviolet irradiation in a laminar flow hood for $30 \mathrm{~min}$. To provide a hydrophilic surface for efficient cell attachment, scaffolds were pre-wetted by immersion in different ethanol concentrations for 30 minutes each $(95 \%, 50 \%$ 
and $25 \%$ ethanol), 30 minutes in distilled water and finally overnight in HBSS buffer in the $37^{\circ} \mathrm{C}$ incubator.

\subsubsection{Chondrogenic differentiation}

Induction of chondrocyte differentiation in high-density cellular aggregates was performed using pellet cultures in accordance with a protocol published previously [21]. To induce chondrogenic differentiation, 250,000 cells (aMSCs or human chondrocytes) were seeded in low attachment 96 round-bottom well plates (Corning). Pellets were formed by centrifugation and maintained for 24 hours in $37{ }^{\circ} \mathrm{C}, 95 \%$ humidity and $5 \% \mathrm{CO}_{2}$ until cells coalesced. Following pellet formation, maintenance media was exchanged with chondrogenic differentiation media, which was replaced every three days.

For PCL scaffolds, aMSCs and human primary chondrocytes grown in T175 cell culture flasks were trypsinized, counted, and plated at a density of 400,000 cells/scaffold. Scaffolds were placed in 24-well culture plates pre-coated with $0.3 \%$ poly-(hydroxyethyl-methacrylate) to prevent cell attachment to the tissueculture plate surface. Scaffolds were incubated at $37^{\circ} \mathrm{C}$ for 4 hours to allow correct migration of the cells trough the nanofibers. During the first 4 hours, 30 $\mu \mathrm{l}$ of complete media was added every 30 minutes to each scaffold to prevent desiccation of the fibers. After 4 hours of incubation $2 \mathrm{ml}$ of complete media was added. Cell viability was monitored using a Live/Dead staining kit (Life Technologies, Carlsbad, CA) at Days 1, 3 and 7 in culture to visualize the live cells attached to the nanofibrous PCL scaffold. Fluorescence observations were performed using a semi-automated ZEISS Axio inverted light microscope with appropriate filters (Zeiss, Oberkochen, Germany). Cell culture medium was replaced every three days.

\subsubsection{Normoxia and hypoxia incubation}

To evaluate the response to oxygen tension of aMSCs and human primary chondrocytes, both cell types cultured in monolayer, pellets and scaffolds were 
allowed to differentiate for up to 21 days in chondrogenic differentiation media under either normoxic ( $21 \%$ oxygen) or hypoxic ( $2.1 \%$ oxygen) conditions using an integrated system with a cell culture hood and incubator accessible through a gas-lock (I-Glove, BioSpherix, New York, NY).

\subsubsection{Gene expression analysis}

Total RNA was isolated using the miRNeasy Micro Kit (Qiagen, Hilden, Germany) following the instructions of the manufacturer. For pellet cultures, at least five pellets were pooled from each condition and lysed using an 18-gauge needle and Qiazol lysis buffer (Qiagen, Hilden, Germany) at each time point. Scaffolds were lysed using the same syringe-needle homogenization method. Lysates of cells used for plating the cells on scaffolds or pellet culture were obtained on day 0 (D0), and at D1, D7 and D14 after culture under various conditions. Biological triplicates were used for analysis. RNA concentrations and purity levels were measured using a NanoDrop (Thermo Fisher Scientific) and isolated RNA was reverse transcribed into cDNA using the SuperScript III First-Strand Synthesis System (Invitrogen, Carlsbad, CA). Gene expression was quantified using quantitative real-time reverse transcriptase polymerase chain reaction (qRTPCR) with primers for representative chondrogenic genes (Table S1). Real-time qPCR reactions were performed with $10 \mathrm{ng}$ cDNA per $10 \mu \mathrm{L}$ with QuantiTect SYBR Green PCR Kit (Qiagen) and the CFX384 Real-Time System (BioRad, Hercules, CA). Gene expression levels were quantified using the $2^{-{ }^{\Delta} \mathrm{Ct}}$ method. Various housekeeping genes were tested for low variation across our samples including GAPDH, ACTB and AKT1. Because the housekeeping gene AKT1 was found to be more stably expressed than GAPDH, we normalized all data relative to $A K T 1$ (mean \pm standard error of the mean, $\mathrm{n}=3$ ). 
Table S1. List of forward and reverse primer sequences used for gene expression analysis.

\begin{tabular}{|l|l|l||}
\hline Official Gene Symbols & Forward primer sequence & Reverse primer sequence \\
\hline AKT1 & GTGCCTATCAGGACAAGGTCT & GATGCCTTTCACCACGACTTC \\
COL10A1 & AAGAATGGCACCCCTGTAATGT & ACTCCCTGAAGCCTGATCCA \\
COL1A1 & GCTACCCAACTTGCCTTCATG & TGCAGTGGTAGGTGATGTTCTGA \\
COL2A1 & TGAAGGTTTCTGCAACATGGA & TTGGGAACGTTTGCTGGATT \\
COMP & AAGAACGACGACCAAAAGGAC & CATCCCCTATACCATCGCCA \\
DCN & ATGAAGGCCACTATCATCCTCC & GTCGCGGTCATCAGGACTT \\
GLI1 & AACGCTATACAGATCCTAGCTCG & GTGCCGTTTGGTCACATGG \\
HAPLN1 & TCTGGTGCTGATTTCAATCTGC & TGCTTGGATGTGAATAGCTCTG \\
HIF1A & TTCCTCTCTCTCCGCGTG & ACTTATCTIITCTTGTCGTCGC \\
HISTH4 & AGCTGTCTATCGGGCTCCAG & CCTTTGCCTAAGCCTTTTCC \\
IHH & AACTCGCTGGCTATCTCGGT & GCCCTCATAATGCAGGGACT \\
SOX9 & TGTATCACTGAGTCATTTGCAGTGT & AAGGTCTGTCAGTGGGCTGAT \\
\hline
\end{tabular}

Note: All primer sequences are indicated from $5^{\prime}$ - end to $3^{\prime}$ end $\left(5^{\prime} \rightarrow 3^{\prime}\right)$.

\subsubsection{Histological analysis}

Cell pellets and scaffolds were fixed overnight in 10\% neutral buffered formalin. Samples were then washed and dehydrated in graded series of ethanol $(70 \%$ $100 \%)$ and processed with Xylene (50\%-100\%) prior to paraffin embedding. Paraffin blocks were cut into consecutive sections of $5 \mu \mathrm{m}$ thickness using a microtome and placed onto charged microscope glass slides for histological staining. Following deparaffinization, sections were stained for glycosaminoglycan content using $0.5 \%$ (wt/vol) Alcian Blue 8GX dye (SigmaAldrich) and counterstained with Nuclear Fast Red (Sigma-Aldrich).

The presence of COL2A1 was localized by immunohistochemistry (IHC). In brief, after removal of paraffin and rehydration, sections were washed with distilled water. Pepsin antigen retrieval was performed using $10 \mathrm{mg} / \mathrm{mL}$ pepsin for 10 minutes at $37^{\circ} \mathrm{C}$ in a humidified chamber. The mouse and rabbit specific HRP (ABC) Detection IHC Kit (Abcam, Cambridge, UK) was used according to the manufacturer's instructions. Briefly, protein block was applied after which slides were incubated overnight with $1 \mu \mathrm{g} / \mathrm{mL}$ of anti-Collagen Type II Antibody (Millipore, Darmstadt, Germany) or mouse IgG1 Isotype Control (Biosciences, Osage, IA) at $4^{\circ} \mathrm{C}$ in a humidified chamber. Slides were sequentially incubated with biotinylated polyvalent secondary antibody and streptavidin peroxidase plus (supplied in the kit) and were then treated with 3, 3'-diaminobenzidine 
(DAB) enhanced liquid substrate system for IHC (Sigma-Aldrich) diluted 1:1 with TBS for 10 minutes until color development. Stained sections were analyzed using a ZEISS Axio inverted light microscope as described above.

\subsubsection{Statistical analysis}

Quantitative data for gene expression profiles have been presented as mean \pm standard deviation from 3 independent donors for aMSCs and one donor for human primary chondrocytes for each experimental condition and time point. The experimental factors analyzed for their association with gene expression values included oxygen level ( 2 levels: normoxia, hypoxia), culture condition (3 levels: 2D monolayer, 3D high-density pellet, 3D PCL scaffold), time (5 levels: baseline and days 1, 3, 7, and 14), and cell type (2 levels: aMSCs, chondrocytes). Each combination of the experimental conditions was performed in triplicate. Separate analyses were performed for each of the 12-targeted genes. The Mayo Clinic Biostatistics Core completed all statistical analysis. The analyses were performed using generalized linear models utilizing generalized estimating equations (GEE) to account for the within-donor correlation among the subsamples from each donor. Significant main effects with more than 2 levels were analyzed further by generating pairwise contrasts to identify levels that are significantly different from each other. In order to protect against an increased type I error rate associated with multiple comparisons, the reported $P$ values were adjusted using the Benjamini-Hochberg method to control the false discovery rate. All analyses were conducted in SAS version 9.4 (SAS Institute Inc., Cary, NC). Statistical analysis is represented as ${ }^{*}$ for $p<0.05$ and as ${ }^{* *}$ for $p<0.0001$. 


\subsection{RESULTS}

\subsection{1 aMSCs undergo chondrogenic differentiation and adopt a phenotype resembling growth plate cartilage}

Expression of chondrogenic markers in differentiated aMSCs cultured in monolayer cultures was compared to primary human chondrocytes to determine whether a chondrocytic phenotype was achieved. aMSCs and chondrocytes exhibited increased expression of several chondrogenic genes over time. Chondrocytes significantly up-regulated ACAN, HAPLN1 and SOX9 over the time course compared to AMSCs (Figure 3.1.A). Surprisingly, differentiating aMSCs exhibited higher levels of gene expression for COL2A1, DCN and COMP and also expression of hypertrophic markers including IHH, GLI1 and COL1OA than chondrocytes (Figure 3.1.B and C). These results suggest that the mechanisms utilized by aMSCs undergoing chondrogenic differentiation are different to those used by primary human chondrocytes, perhaps reflecting adoption of phenotypes resembling growth plate cartilage rather than articular cartilage.

\subsubsection{Culture on PCL-scaffolds supports cartilage-specific extracellular matrix formation by both aMSCs and chondrocytes}

To aid in the clinical translation of aMSCs for the treatment of cartilage defects, we investigated whether a 3D nanofibrous PCL scaffolds could support chondrogenic differentiation of aMSCs. Cells seeded on scaffolds were compared to those seeded on high-density pellet cultures. After fabrication, scaffolds were visualized by light microscopy and were observed to be homogenous in size and shape (Figure 3.2.A). aMSCs were seeded onto the scaffold and live/dead staining revealed that the cells attached and proliferated within the scaffold after 1, 3, 7 and 14 days. At all-time points, the majority of cells were viable (green) with few non-viable cells (red). Moreover, fluorescence microscopy at day 14 revealed cells were tightly packed indicating cells reached a confluent state (Figure 3.2.B). 

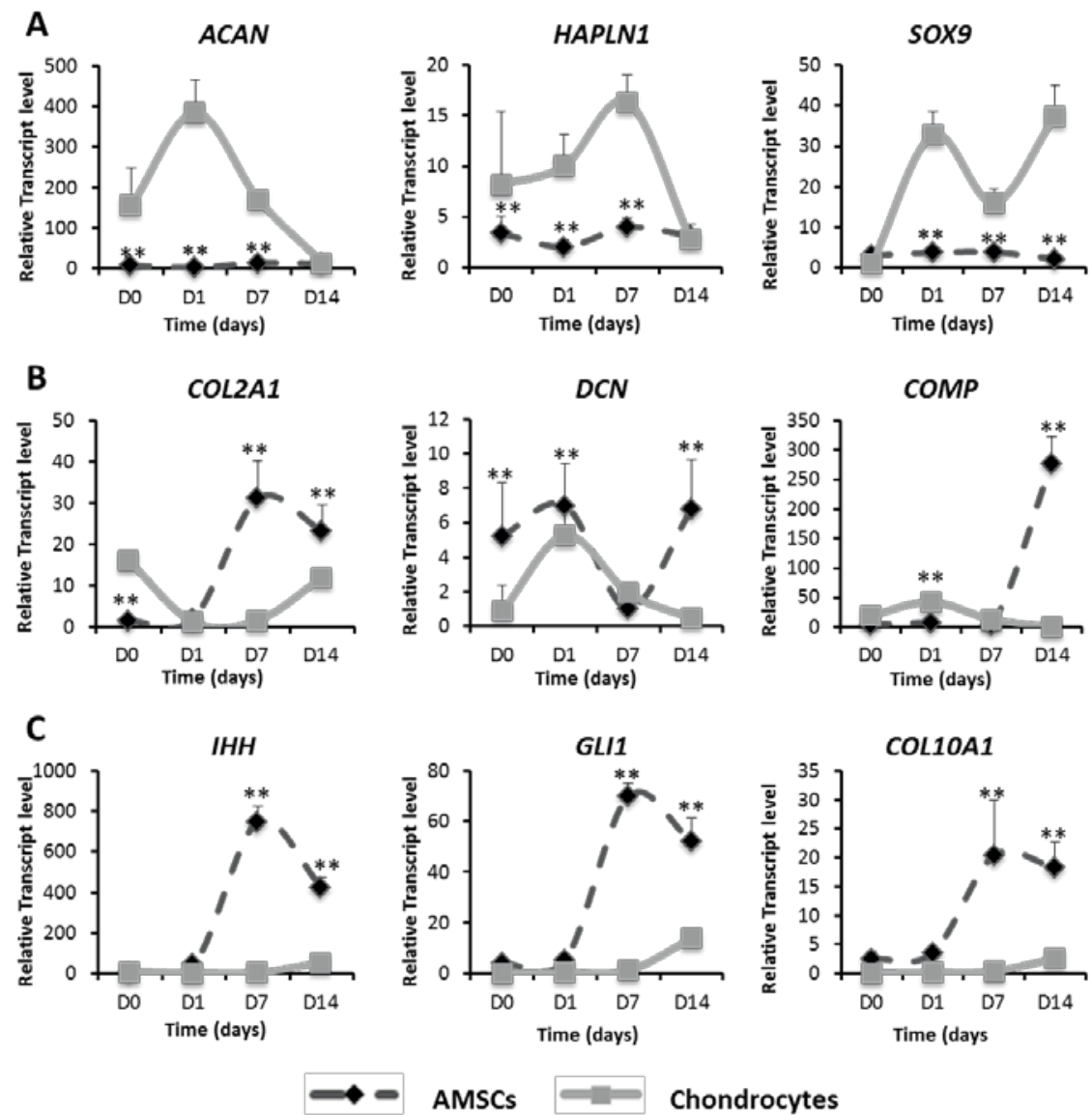

\section{Chondrocytes}

Figure 3.1. Gene expression profiles of aMSCs and human primary chondrocytes in monolayer cultures during chondrogenic differentiation. (A) Earlier and robust expression of ECM proteins ACAN and HAPLN1, and transcription factor SOX9 was observed in human primary chondrocytes over the chondrogenic time course. (B) aMSCs showed an increased expression of COL2A1 while up-regulating ECM proteins $D C N$ and COMP when compared to human primary chondrocytes. (C) Hypertrophic phenotype is induced in aMSCS over time, which is evidenced by the up-regulation of $I H H, G L I 1$, and COL1OA1 over time in aMSCs. Data are presented as mean \pm standard error of the mean, $n=3$. For RT-qPCR three technical replicates were used. Statistical analysis is represented as

* for $p<0.05$ and as ** for $p<0.0001$.

Gene expression analysis revealed differences between the pellet culture and scaffold system. Compared to pellet cultures, 3D-scaffolds did not promote expression of chondrogenic ECM markers including HAPLN1, COMP, ACAN, 82 
COL2A1 and the transcription factor SOX9 (Figure 3.2.C, D, E, F and G). Also, scaffold cultures did not enhance expression of HIST2H4 and HIF1A, which was evident in pellet cultures (Figure 3.2.H and I). Significant up-regulation of HIF1A in pellet cultures suggests a hypoxic microenvironment within the pellet even when cultured under regular oxygen conditions. This upregulation was not seen in cells cultured in scaffolds. Moreover, expression of hypertrophic markers $\mathrm{IHH}$ and COL1OA1 were down regulated in scaffold cultures throughout the time course (Figure 3.2.J and $\mathrm{K}$ ). DCN protein was the only chondrogenic marker to show a similar expression in both pellet and scaffold cultures (Figure 3.2.L). In contrast, osteogenic/fibroblastic marker COL1A1 and hypertrophic marker GLI1 were increased in scaffolds over pellets by 2.9 -and 1.09 fold respectively (Figure

\subsection{M and $N$ ).}

Histological analysis of aMSCs cultured in scaffolds and pellets at day 21 of chondrogenic differentiation indicated that both cultures permit development of an Alcian blue positive matrix (Figure 3.2.0, $a$ and $\boldsymbol{d}$ ). However, the matrix was denser in pellet cultures (Figure 3.2.0, b and $\boldsymbol{c}$ ). In scaffolds, cells were located at the periphery indicating poor cell migration towards the scaffold center (Figure 3.2.0, e and f). To validate the expression of COL2A1 was detected by IHC in pellets at day 21 of culture (Figure 3.2.P). In summary, scaffold increased hypertrophic marker expression and osteogenic potential of the aMSCs when compared to pellet cultures. Chondrogenic differentiation of aMSCs in pellet cultures promoted the expression of cartilage-like ECM production, proliferation and a hypoxic microenvironment. Chondrogenic differentiation in either pellets or on scaffolds using human primary chondrocytes was also examined. Chondrogenic markers COL2A1 and ACAN, hypertrophic markers IHH and GLI1, and osteogenic marker COL1A1 gene expression were similar in both 3D culture conditions (Figure 3.3). However, chondrocytes ECM proteins HAPLN1 and COMP were enhanced in 3D-scaffolds when compared to pellets (Figure 3.3.A and $B$ ). 

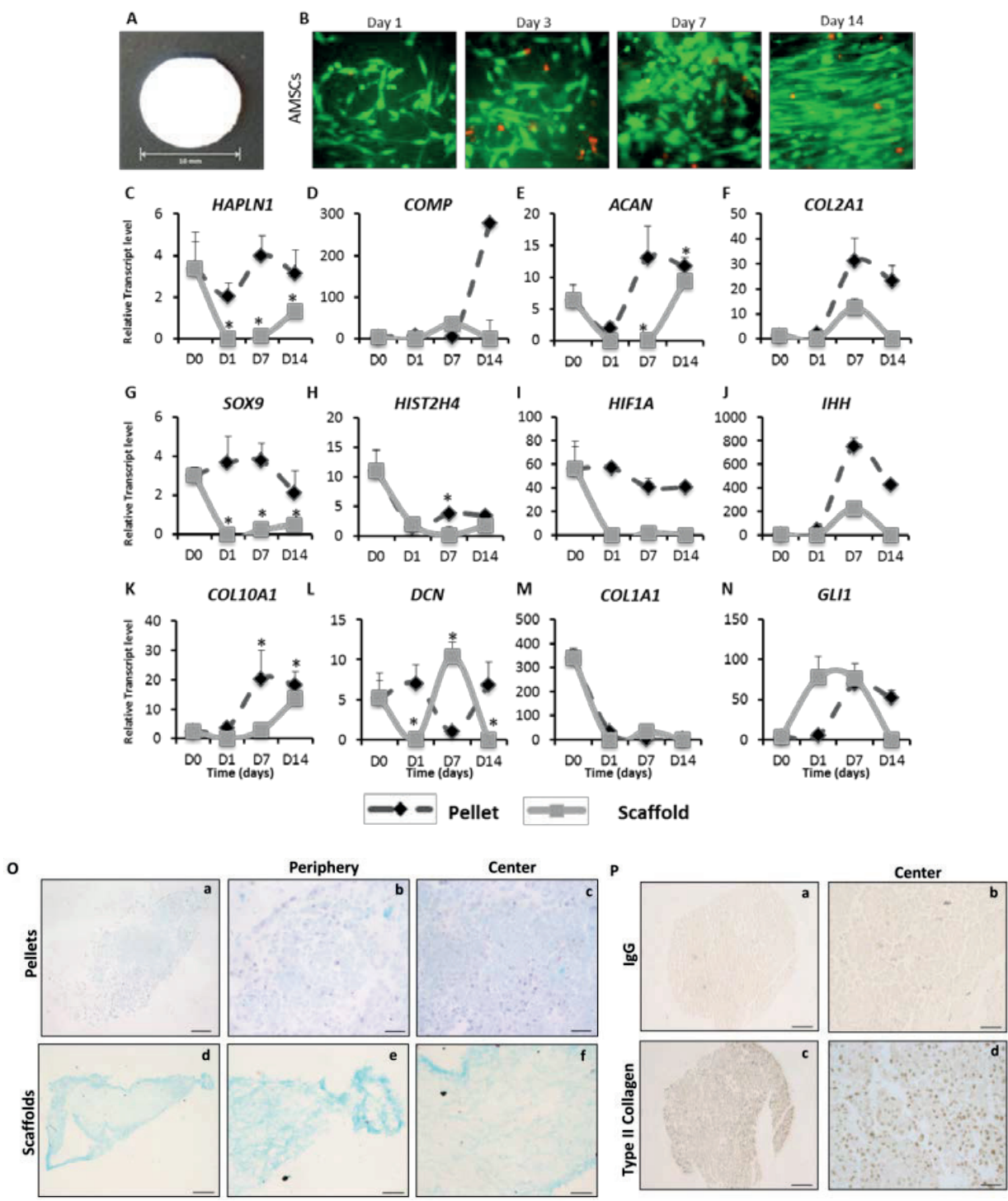

Figure 3.2 Gene expression and histological analysis differences between PCL-scaffold and highdensity pellets cultures using AMSCs. (B) Live(green)/dead(red) analysis showing aMSCs attached and proliferated on the scaffold. (C to $\mathbf{N}$ ) Gene expression analysis of chondrogenic, proliferative and fibroblastic markers. Data is presented as mean \pm standard error of the mean, $n=3$. For RTqPCR three technical replicates were used. Statistical analysis is represented as * for $p<0.05$ and ** for $p<0.0001$. (0) Alcian blue staining of aMSCs in either PCL-scaffolds or high-density pellet cultures at day 21 of chondrogenic differentiation. (Scale bar $=100 \mu \mathrm{m}$ ). (P) COL2A1 detected by immunohistochemistry in pellets at day 21 of culture, shown at $10 \mathrm{x}$ and $40 \mathrm{x}$ magnification. 
Scaffold cultures down-regulated expression of SOX9, DCN, COL10A1, HIF1A and HIST2H4 when compared to high-density pellets (Figure 3.3.E to J). Histological analysis showed alcian blue staining in chondrocytes when cultured in both 3D conditions at day 21 (Figure 3.3.M). Within pellets, cells were located in both the periphery and the center and a dense matrix was observed throughout the pellet (Figure 3.3.M, b and c). In scaffolds, poor migration of the cells within the scaffold was observed with aMSCs (Figure 3.3.M, e and f). Chondrocytes did not show any specific orientation or arrangement within the pellet or scaffold. A light expression of COL2A1 was detected by IHC in pellets at day 21 of culture (Figure 3.3.N), indicating a chondrogenic matrix. Together, these results suggest that both high-density pellets and PCL-scaffolds cultures are conducive to the production of a glycosaminoglycan positive matrix, and the cartilage-like gene expression profile indicates that the chondrocytic phenotype was preserved.

Gene expression analysis revealed similarities between aMSCs and chondrocytes under both 3D culture conditions. Scaffold cultures did not support the expression of chondrogenic transcription factor SOX9, hypertrophic marker COL10A1, hypoxia inducible factor HIF1A and HIST2H4 in both aMSCs and chondrocytes. Also, ECM protein DCN mRNA levels were decreased in scaffolds when compared to pellets at day 1 in both AMSCs and chondrocytes. However, chondrogenic markers COL2A1 and ACAN were different in both cell cultures. Although the majority of the gene expression patterns look similar, the levels of expression varied between cells. Chondrocyte cultures expressed higher mRNA levels of SOX9, ACAN, COMP and HAPLN1 whereas aMSCS expressed higher levels of COL2A1 and hypertrophic genes. 

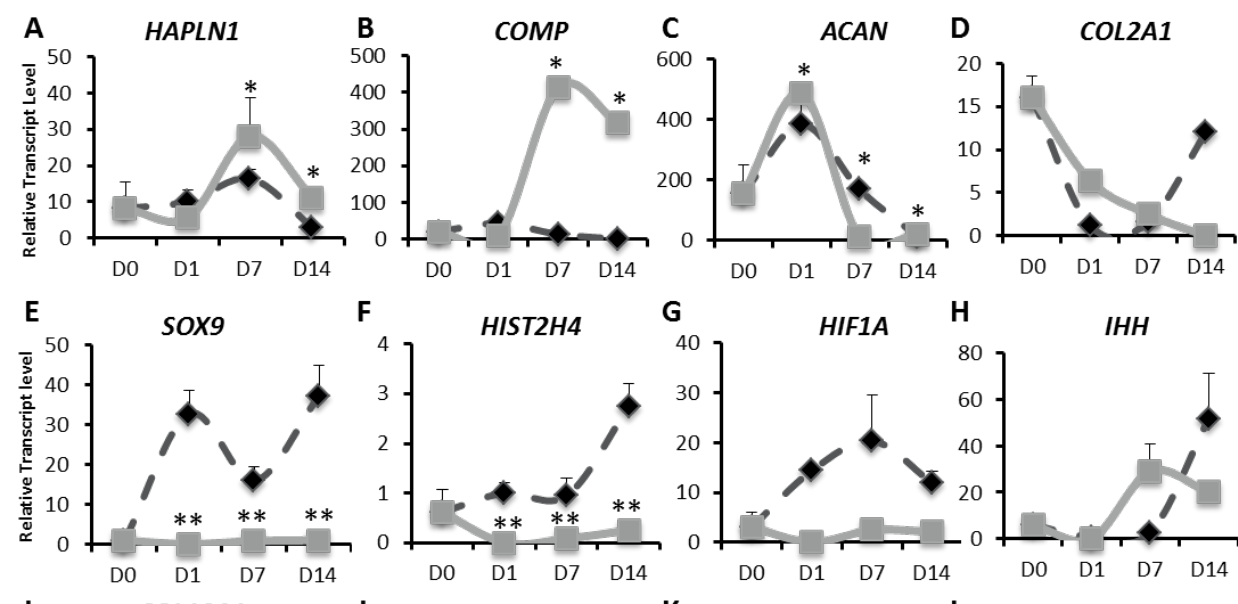

F HIST2H4 G
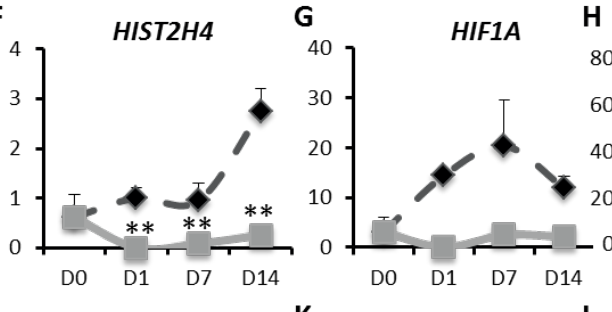

IHн
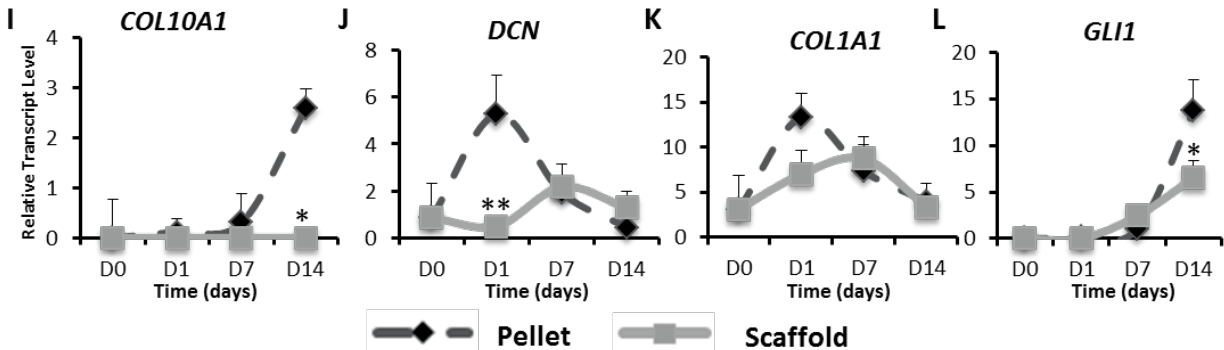

Scaffold
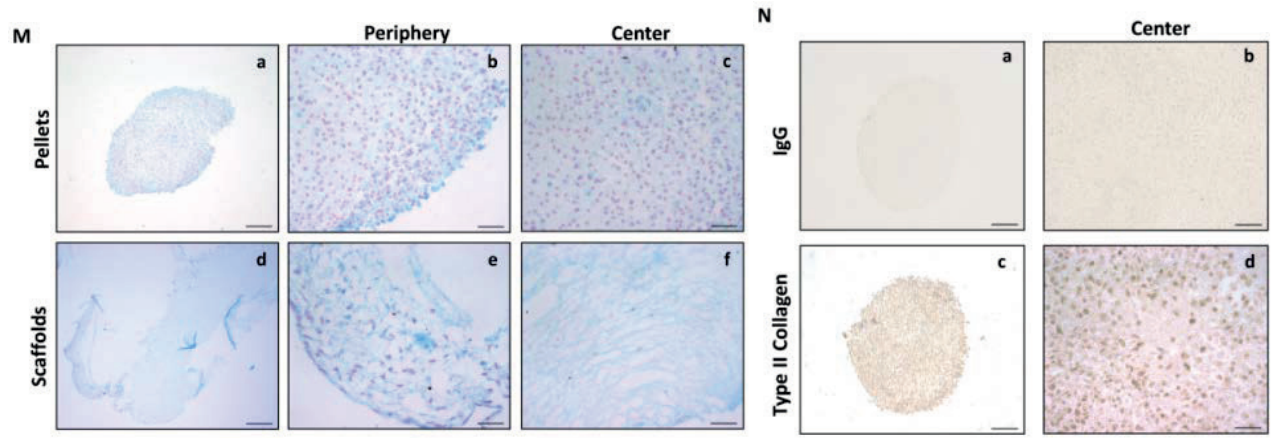

Figure 3.3. Analysis of human primary chondrocytes differentiated in 3D-scaffold cultures and

high-density pellets. (A to L) Gene expression analysis of chondrogenic, proliferative and fibroblastic markers. Data is presented as mean \pm standard error of the mean, $n=3$. For RT-qPCR three technical replicates were used. Statistical analysis is represented as * for $p<0.05$ and ${ }^{* *}$ for $p<0.0001$. (M) Alcian blue staining of primary chondrocytes cultured in 3D-scaffolds cultures and high-density pellets at day 21 of chondrogenic differentiation (Scale bar $=100 \mu \mathrm{m})$. (N) COL2A1 detected by IHC in pellets at day 21 of culture, shown at 10x and 40x magnification. 


\subsubsection{Hypoxia promotes cartilage-specific gene expression in aMSCs in both pellets and PCL-scaffolds}

To determine if low oxygen tension enhances chondrogenic differentiation, we compared pellet cultures of both aMSCs and chondrocytes in normoxia $\left(21 \% \mathrm{O}_{2}\right)$ or hypoxia $\left(2.1 \% \mathrm{O}_{2}\right)$. Gene expression analysis revealed that while the expression of a number of cartilage related genes was generally independent of oxygen levels, several cell type-specific responses to hypoxia were observed. In aMSCs, normoxia stimulated the expression of ECM proteins ACAN, COL2A1 (Figure 3.4.A and B) and DCN (data not shown) after one week of culture. Conversely, hypoxia stimulated the preferential expression of the proliferation marker HIST2H4 as well as HIF1A, which is the master regulator of the cellular response to hypoxia [22, 23] (Figure 3.4.C and D).

Low oxygen conditions also increased levels of hypertrophic mRNA markers COL10A1 (Figure 3.4.E), as well as GLI1 and IHH (data not shown). A reduced oxygen environment accelerated down-regulation of COL1A1, thus decreasing the osteogenic and/or fibroblastic potential of chondrocytes (Figure 3.4.F). Moreover, hypoxia increased the expression of the chondrogenic transcription factor SOX9 (1.6 fold, day 3), HAPLN1 (2.1 fold, day 3) (Figure 3.4.G and $\boldsymbol{H}$ ) and COMP (1.8 fold, day 1, data not shown) compared to normoxia in aMSCs. Our studies also revealed that despite phenotypic differences between aMSCs and chondrocytes, hypoxia enhanced expression of HIST2H4 and a sligh upregulation of HIF1A on day 1 in both cells. The latter finding suggests that low oxygen and HIF1A protein may play an important role in regulating cell proliferation and survival earlier during differentiation.

We also evaluated whether oxygen tension modulated chondrogenic differentiation of aMSCs and chondrocytes cultured on scaffolds. Gene expression analysis revealed similarities between aMSCs and chondrocytes in both low and high oxygen cultures, although cell type specific differences were observed. 

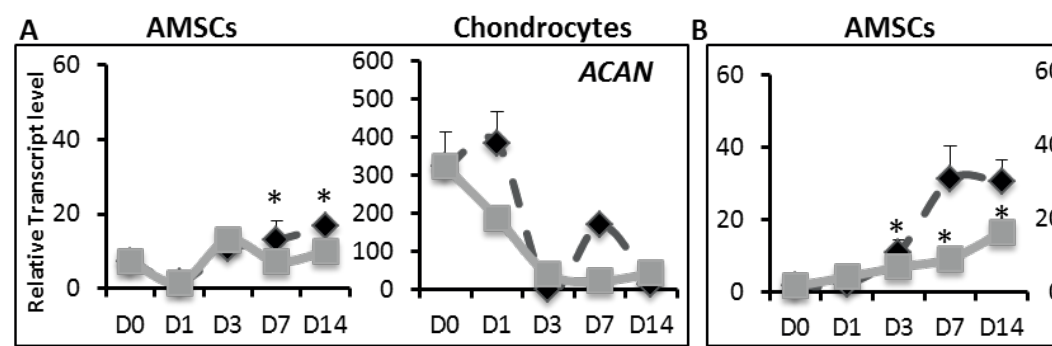

Chondrocytes
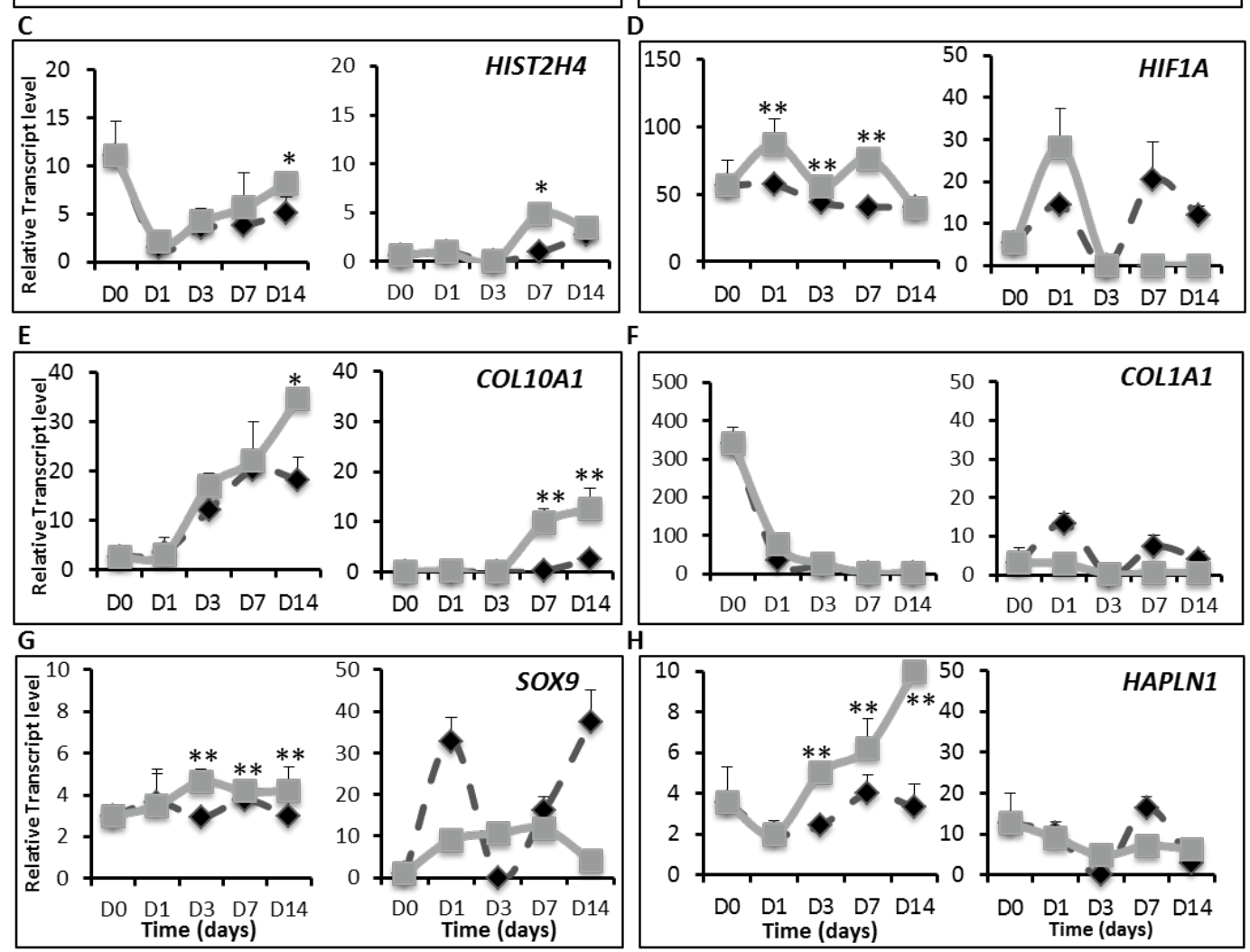

$\rightarrow$ - Normoxia
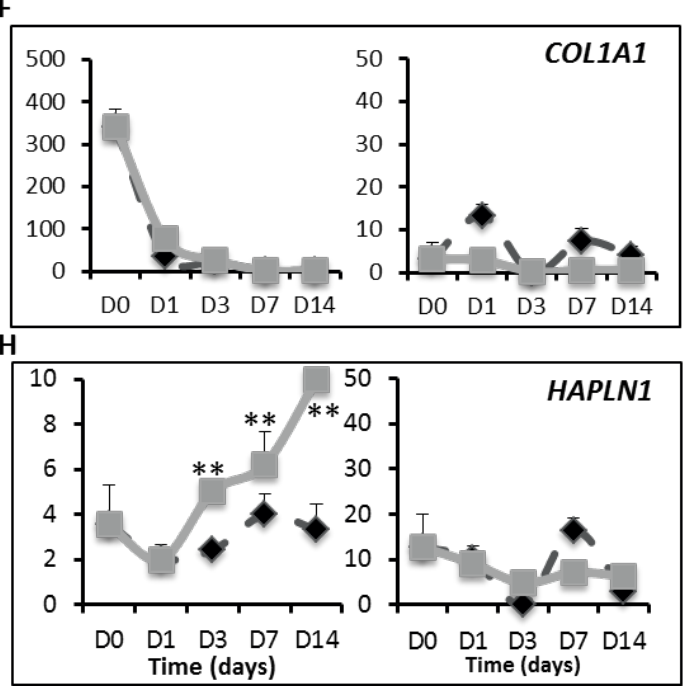

Hypoxia

Figure 3.4. The effect of oxygen tension on differentiation potential of aMSCs and chondrocytes cultured in high-density pellets. aMSCs and chondrocytes were placed in pellet cultures and were exposed to normoxic $\left(20 \% \mathrm{O}_{2}\right)$ or hypoxic conditions $\left(2.1 \% \mathrm{O}_{2}\right)$ for 14 days. Gene expression analysis of chondrocyte specific markers, hypoxia related factor protein $H I F 1 A$ and hypertrophic markers was performed at days $0,1,3,7$ and 14. Data is presented as mean \pm standard error of the mean, $n=3$. For RT-qPCR three technical replicates were used. Statistical analysis is represented as

$$
\text { * for } p<0.05 \text { and }{ }^{* *} \text { for } p<0.0001 \text {. }
$$


In aMSCs differentiated on scaffolds, hypoxia increased the expression of chondrogenic markers ACAN (Figure 3.5.A), SOX9 (Figure 3.5.G) and HAPLN1 (Figure 3.5.H) whereas normoxia up-regulated osteogenic/fibroblastic marker COL1A1 (Figure 3.5.B) and hypertrophic markers COL10A1 (Figure 3.5.E), GLI1 and $I H H$ (data not shown). Normoxia also increased mRNA levels of COL2A1 at day 7 (Figure 3.5.F). In chondrocytes, hypoxia up-regulated the expression of transcription factor SOX9 (Figure 3.5.G), HIST2H4 and HIF1A (Figure 3.5.C and D), while normoxia increased COL1A1 (Figure 3.5.B). Interestingly, low oxygen tension enhanced expression of chondrogenic genes in chondrocytes that were repressed in aMSCs, including COL2A1 (Figure 3.5.F), DCN and GLI1 and IHH (data not shown). Conversely, chondrocytes on scaffolds cultured under normoxic conditions up-regulated several chondrogenic genes including ACAN (Figure 3.5.A) and HAPLN1 (Figure 3.5.H). The hypertrophic marker COL10A1 was the only gene that was not expressed by chondrocytes grown on scaffolds (Figure 3.5.E). In summary, low oxygen conditions enhance the expression of several chondrogenic markers of aMSCs cultured on scaffolds, but chondrocytes were largely unaffected by differences in oxygen tension levels.

\subsection{DISCUSSION}

Tissue engineering offers possibilities for optimization of cartilage repair by combining different cell types, biomaterials, and growth factors for the support of cartilage regeneration [24]. Although several procedures have been developed and studied, no standardized clinical protocol has yet been established [25]. The use of stem cells and in particular mesenchymal stem cells from adult sources has emerged as a viable solution to overcome limitations of current cartilage repair procedures.

The chondrogenic potential of clinical-grade and human platelet lysate-expanded human aMSCs under multiple culture conditions relevant to cartilage tissue engineering, including hypoxia and propagation in a 3D culture environment. 

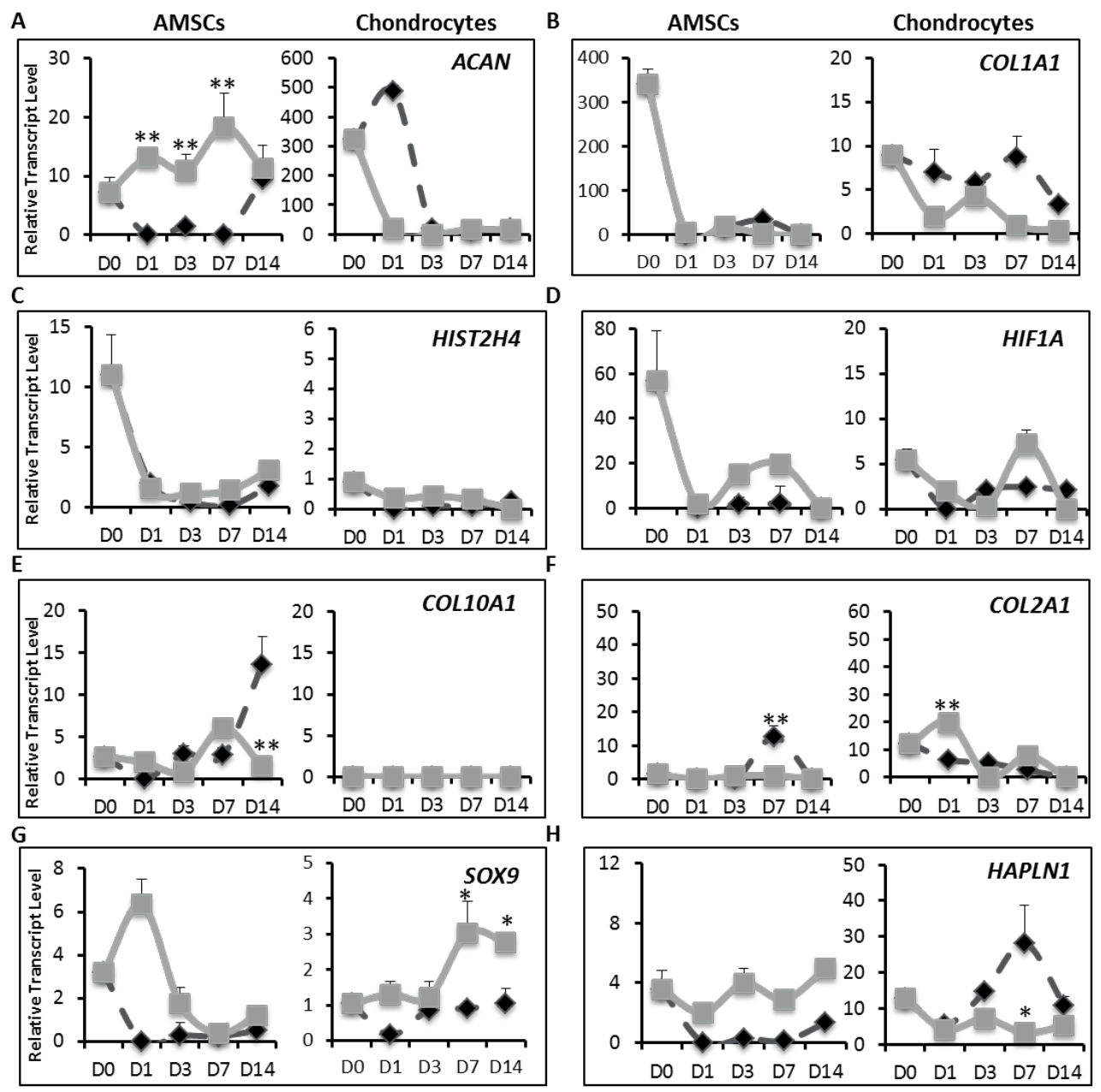

$\longrightarrow$ - Normoxia

Hypoxia

Figure 3.5. The effect of oxygen tension on differentiation potential of aMSCs and primary human chondrocytes cultured in PCL-scaffolds. PCL-scaffolds were seeded with either aMSCs or chondrocytes and placed under hypoxic or normoxic conditions in the presence of chondrogenic differentiation medium for 14 days. Gene expression analysis was performed at days $0,1,3,7$ and

14. Data is presented as mean \pm standard error of the mean, $n=3$. For RT-qPCR three technical replicates were used. Statistical analysis is represented as * for $p<0.05$ and ** for $p<0.0001$.

While there are many studies on chondrogenic differentiation of bone marrow derived MSCs, our studies address a major gap in our knowledge by investigating Most of the previous studies have been performed using animal serum (FBS), 
which is not applicable for clinical studies. In our case, human platelet lysate presents a non-xenogeneic option for cell expansion and differentiation of aMSCs for translational studies. This study provides a detailed side-by-side comparison of aMSCS and human primary chondrocytes during chondrogenic differentiation, which was not previously available in the literature. Furthermore, current protocols for chondrogenic differentiation efficiently create hypertrophic cartilage which upon implantation undergoes endochondral ossification and mineralization [26]. Several studies have attempted to understand the mechanisms by which MSCs can be programmed for chondrogenic differentiation while avoiding further progression into hypertrophic cartilage [27-29]. Mimicking the natural hypoxic environment of cartilage development under in vitro conditions is beneficial because it mediates metabolic programming of the chondrogenic fate of MSCs into different subtypes of hyaline cartilage. The results presented in this study validate the biological properties of self-renewing and differentiation potential of aMSCs. However, a need for better chondrogenic differenaition strategies that avoid a hypertropic phenotype of the cells is still required.

The comparison between normoxia and hypoxia of aMSCs and chondrocytes cultured in pellets revealed an enhanced expression of chondrogenic markers that were expressed in both oxygen conditions. Previous studies have described neo-hyaline cartilage tissue formation demonstrated by increased COL2A1 expression and higher glycosaminoglycan content [30]. Furthermore, extracellular matrix proteins (e.g., ACAN, DCN, COMP and HAPLN1) are highly expressed in human cartilage and human articular chondrocytes [17, 31, 32]. In our studies, both cell types respond to hypoxia by enhancing the expression of HIST2H4, HIF1A and COL2A1, suggesting that HIF1A plays an important role in regulation of earlier cell proliferation and the expression of COL2A1 under low oxygen conditions. As a consequence of continuous hypoxia under chondrogenic induction, stem cells start to produce hyaline cartilage that is resistant to 
hypertrophic differentiation, whereas incubation under normoxia conditions results in hypertrophic cartilage that resembles epiphyseal cartilage [31,33]. Our data indicates that under chondrogenic induction aMSCs develop a chondrocytelike phenotype, but may have a propensity to exhibit a hypertrophic response despite being exposed to a hypoxic environment. In contrast, Leijten et al. showed that hypoxia cultures of bone marrow derived MSCs increased chondrogenic differentiation while avoiding hypertrophic differentiation of the cells [21]. Furthermore, our study suggests that the molecular response observed in aMSCs undergoing chondrogenic differentiation is different from the molecular response by primary human chondrocytes, in particular because aMSCs may adopt a phenotype resembling growth plate cartilage rather than articular cartilage.

Oxygen responsiveness and chondrogenic potential also appear to be affected by the choice of cell culture model. Several cell type independent effects in gene expression in high-density pellets versus scaffolds and cell type specific expression were observed. In the current investigation, we found that changing the cell environment from a two-dimensional culture to a 3D culture model (i.e., high density pellets or PCL-scaffold), improved the chondrogenic potential of aMSCs and primary chondrocytes by increasing cell-cell interactions and generating a low oxygen environment within cellular aggregates. We observed that conventional monolayer culture did not support the chondrogenic differentiation of aMSCs, whereas both high-density pellets and scaffolds cultures are conducive to the production of a glycosaminoglycan positive matrix. A cartilage-like gene expression profile indicates that aMSCs acquire a chondrocytic phenotype but is not stable. Moreover, pellet culture promoted proliferation and a hypoxic microenvironment, whereas the scaffold increased hypertrophic marker expression and osteogenic potential of the aMSCs. Our results using nanofibrous $P C L$ scaffolds corroborate previous studies and 
support the chondrogenic differentiation potential of MSCs when cultured under appropriate inductive conditions [34].

The potential use of scaffolds for implantation relies on their flexible designs, excellent biomechanical properties and slow degradation rate. There is evidence that MSCs favor nanotopographical geometry and porous materials over plastic for growth and differentiation [24, 34]. Scaffolds have been largely used to provide cells with structural support for attachment, proliferation, and differentiation in a 3D environment. Moreover, scaffolds facilitate optimal filling of the entire defect and provide mechanical support for the healing tissue, potentially reducing the rehabilitation time for the patient $[35,36]$. A combination of a biodegradable scaffold with stem cells would better resemble the native tissue composition and mechanical properties and could potentially regenerate hyaline cartilage tissue. For articular cartilage surface regeneration, several scaffolds have been used including nanomaterials, functional mechanocompatible scaffolds, multi-layered scaffolds, and ECM scaffolds [34]. Some of these scaffolds can cover the entire chondral defect but their use is typically accompanied by production of a COL1A1 rich ECM, thus producing a biomechanical inferior and non-durable matrix that does not resemble native cartilage $[37,38]$. In our studies, low oxygen cultures repressed the expression of fibroblastic/osteogenic marker COL1A1 in both aMSCs and chondrocytes on both 3D-cultures. Taken together, the results presented here support the important role of oxygen regulation in chondrogenic differentiation of aMSCs for clinical applications.

The cell type specific role of low oxygen in differentiation of aMSCs indicates that mesenchymal cell fate and differentiation potential is remarkably sensitive to oxygen. The oxygen status is a dominant environmental parameter for directing chondrogenic differentiation of aMSCs and reduces their tendency to generate a fibroblastic ECM, while helping chondrocytes to maintain their phenotype by enhancing COL2A1 expression and inhibiting additional matrix production. 
Hence, hypoxia is more effective in promoting a chondrocytic phenotype at earlier stages of differentiation. Our findings indicate that genetic programming of aMSCs to a chondrocytic phenotype is effective in cellular aggregates (pellet culture) under hypoxic conditions as reflected by increased expression of cartilage-related biomarkers and biosynthesis of a glycosaminoglycan positive matrix. Although expression of cartilage-related genes is more robust in pellets than scaffolds, hypoxia enhances the low levels observed in scaffold cultures. Our results provide a new starting point for optimization of aMSCs in cartilage engineering strategies that leverage the chondrogenic effects of hypoxia in the 3D environment of an implantable scaffold for treatment of focal cartilage defects. 


\section{REFERENCES}

1. Falah M, Nierenberg G, Soudry M, Hayden M and Volpin G: Treatment of articular cartilage lesions of the knee Int Orthopedic 2010:34:621-630.

2. Heng BC, Cao T, Lee EH: Directing stem cell differentiation into the chondrogenic lineage in vitro. Stem cells 2004:22:1152-1167.

3. Murtaugh LC, Chyung JH, Lassar AB: . Sonic hedgehog promotes somitic chondrogenesis by altering the cellular response to BMP signaling. Genes Development 1999:13:225-237.

4. Pintan GF, de Oliveira AS, Lenza M, Antonioli E, Ferreti M: Update on biological therapies for knee injuries: osteoarthritis Current Reviews in Musculoskeletal Medicine 2014:7:263-269.

5. Claerbout MT, Matricali GA: Technique tip: modification of a long-leg cast for use in patients with functional impairment or muscle weakness of the upper extremities. Foot Ankle Int 2004:25:176.

6. Brittberg M, Lindahl A, Nilsson A, Ohlsson C, Isaksson O, Peterson L: Treatment of deep cartilage defects in the knee with autologous chondrocyte transplantation. N Engl J Med 1994:331:889-395.

7. Marcacci M, Kon E, Delcogliano M, Filardo G, Busacca M, Zaffagninin: Arthroscopic autologous osteochondral grafting for cartilage defects of the knee: prospective study results at a minimum 7-year follow-up. Am J Sports Med 2007:35:2014-2021.

8. Brittberg M: Autologous chondrocyte transplantation. Clin Orthop Relat Res 1999:367:147-355.

9. Hunziker EB: Articular cartilage repair: basic science and clinical progress. A review of the current status and prospects. Osteoarthritis Cartilage 2002, 10:432-463.

10. Benya PD, Padilla SR, Nimni ME: Independent regulation of collagen types by chondrocytes during the loss of differentiated function in culture. Cell 1978:15:13131321.

11. Lin Z, Fitzgerald JB, Xu J, Willers C, Wood D, Grodzinsky AJ, Zheng MH.: Gene Expression Profiles of Human Chondrocytes during Passaged Monolayer Cultivation. J Orthop Res 2007:26:1230-1237.

12. Harris JD, Siston RA, Pan X, Flanigan DC: Autologous chondrocyte implantation: a systematic review. J Bone Joint Surg Am 2010:92:2220-2233.

13. Song JQ, Dong F, Li X, Xu CP, Cui Z, Jiang N, Jia JJ, Yu B: Effect of treadmill exercise timing on repair of full-thickness defects of articular cartilage by bone-derived mesenchymal stem cells: an experimental investigation in rats. PLoS One 2014:9.

14. Gir P, Oni G, Brown SA, Mojallal A, Rohrich RJ: Human adipose stem cells: current clinical applications. Plastic and reconstructive surgery 2012, 129:1277-1290.

15. Veriter S, Andre W, Aouassar N, Poitel HA, Lafosse A, Docquier PL, Dufrane D.: Human Adipose-Derived Mesenchymal Stem Cells in Cell Therapy: Safety and Feasibility in Different "Hospital Exemption" Clinical Applications. PLoS One 2015, 10:e0139566.

16. Dudakovic A, Camilleri E, Riester SM, Lewallen EA, Kvasha S, Chen X, Radel DJ, Anderson JM, Nair AA, Evans JM: High-resolution molecular validation of self-renewal and spontaneous differentiation in clinical-grade adipose-tissue derived human mesenchymal stem cells. Journal of cellular biochemistry 2014, 115:1816-1828. 
17. Dudakovic A, Camilleri E, Riester SM: High-resolution molecular validation of self-renewal and spontaneous differentiation in clinical-grade adipose-tissue derived human mesenchymal stem cells. J Cell Biochem 2014:115:1816-1828.

18. Crespo-Diaz R, Behfar A, Butler GW, Padley DJ, Sarr MG, Bartunek J, Dietz AB, Terzic A.: Platelet lysate consisting of a natural repair proteome supports human mesenchymal stem cell proliferation and chromosomal stability. Cell Transplant 2011:20:797-811.

19. Schagemann JC, Paul S, Casper M, Rohwedel J, Kramer J, Kaps C, Mittelstaedt H, Fehr M, Reinholz GG.: Chondrogenic differentiation of bone marrow-derived

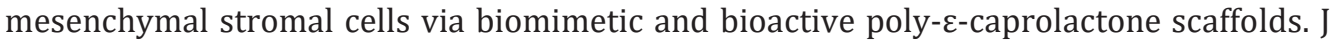
Biomed Mater Res A 2013:101:1620-1628.

20. Leijten J, Georgi N, Moreira Teixeira L, van Blitterswijk CA, Post JN and Karperien M: Metabolic programming of mesenchymal stromal cells by oxygen tension directs chondrogenic cell fate. . Proc Natl Acad Sci U S A 2014:111:13954-13959.

21. Wang GL, Jiang BH, Semenza GL: Effect of protein kinase and phosphatase inhibitors on expression of hypoxia-inducible factor 1. Biochem Biophys Res Commun 1995:216: 669-275.

22. Iyer NV, Kotch LE, Agani F, Leung SW, Laughner E, Wenger RH, Gassmann M, Gearhart JD, Lawler AM, Yu AY, Semenza GL: Cellular and developmental control of 02 homeostasis by hypoxia-inducible factor 1 alpha. Genes Development 1998:12: 149-162.

23. Heng BC, Cao T, Lee EH: Directing stem cell differentiation into the Chondrogenic lineage in vitro. Stem Cells 2004:22: 1152-1167.

24. Fan CM, Porter JA, Chiang C, Chang DT, Beachy PA, Tessier-Lavigne M: Longrange sclerotome induction by sonic hedgehog: direct role of the amino-terminal cleavage product and modulation by the cyclic AMP signaling pathway. Cell 1995: 81: 457-465.

25. van Gool SA, Emons J, Leijten J, Decker E, Sticht C, van Houwelingen JC, Goeman JJ, Kleijburg C, Scherjon SA, Gretz N, Wit JM, Rappold G, Post JN, Karperien M: Fetal mesenchymal stromal cells differentiating towards chondrocytes acquire a gene expression profile resembling human growth plate cartilage. PLoS One 2012:7: e44561.

26. Wu L, Leijten J, Georgi N, Post JN, van Blitterswijk CA, Karperien M.: Trophic effects of mesenchymal stem cells increase chondrocyte proliferation and matrix formation. Tissue Eng Part A 2011:17:1425-1436.

27. Ma B, Leijten J, Wu L, Kip M, van Blitterswijk CA, Post JN, Karperien M: Gene expression profiling of dedifferentiated human articular chondrocytes in monolayer culture. Osteoarthritis Cartilage 2013, 21:599-603.

28. Wu L, Leijten J, Georgi N, Post JN, van Blitterswijk CA, Karperien M: Trophic effects of mesenchymal stem cells increase chondrocyte proliferation and matrix formation. Tissue engineering Part A 2011, 17:1425-1436.

29. Chang NJ, Lin Y, Lin C, Wang HC, Hsu HC, Yeh ML.: The repair of full-thickness articular cartilage defect using intra-articular administration of N-acetyl-D-glucosamine in the rabbit knee: randomized controlled trial. Biomed Eng Online 2015:14:105.

30. Leijten J, Georgi N, Moreira Teixeira L, van Blitterswijk CA, Post JN, Karperien M: Metabolic programming of mesenchymal stromal cells by oxygen tension directs chondrogenic cell fate. Proceedings of the National Academy of Sciences of the United States of America 2014, 111:13954-13959. 
31. Shafiee A, Kabiri M, Langroudi L, Soleimani M, Ai J.: Evaluation and comparison of the in vitro characteristics and chondrogenic capacity of four adult stem/progenitor cells for cartilage cell-based repair. J Biomed Mater Res A 2015.

32. Georgi N, Cillero-Pastor B, Eijkel GB, Periyasamy PC, Kiss A, van Blitterswijk C, Post JN, Heeren RM, Karperien M.: Differentiation of mesenchymal stem cells under hypoxia and normoxia: lipid profiles revealed by time-of-flight secondary ion mass spectrometry and multivariate analysis. Anal Chem 2015, 87:3981-3988.

33. Casper ME, Fitzsimmons J, Stone J, Meza A0, Huang Y, Ruesink TJ, O'Driscoll SW, Reinholz GG.: Tissue engineering of cartilage using poly-epsilon-caprolactone nanofiber scaffolds seeded in vivo with periosteal cells. Osteoarthritis Cartilage 2010:18: 981-991.

34. Georgi N, van Blitterswijk C, Karperien M: Mesenchymal stromal/stem cell or chondrocyte-seeded microcarriers as building blocks for cartilage. Tissue Engineering. Tissue Engineering: Part A 2014:20.

35. Malda J, Frondoza CG.: Microcarriers in the engineering of cartilage and bone. Trends Biotechnology 2006:24:299.

36. Ng YC, Butler, M: Optimization of physical parameters for cell attachment and growth on macroporous microcarriers. Biotechnol Bioeng 2006:50:627.

37. Mastbergen SC, Saris DB, Lafeber FP: Functional articular cartilage repair: here, near, or is the best approach not yet clear?. Nat Rev Rheumatol 2013:9:277-290. 


\section{CHAPTER 4}

4. Integrated analysis of miRNA-seq and mRNA-seq on hypoxic mesenchymal stem cells reveals the role of hypoxiaregulated miRNAs for promoting chondrogenesis of MSCs. 


\section{ABSTRACT}

An optimal tissue engineering strategy that regenerates the natural cartilage structure is required for a successful cartilage tissue repair. Hypoxia, a common environmental condition, influences cell signals and functions [1], and has been suggested to maintain cartilage homeostasis by inhibiting angiogenesis and hypertrophic differentiation, while inducing several genes required for matrix production such as glycosaminoglycans [2] and COL2A1 during chondrocyte development [3]. Several studies have unraveled a complex molecular network that is involved in regulating the hypoxic response in physiological conditions [4]. Although it is believed that under hypoxic conditions an appropriate stimulus could drive chondrogenesis of MSCs, the exact mechanisms are not known. During the past decade, multiple studies have demonstrated that miRNAs are involved in the hypoxic response and contribute to the repression of specific genes under low oxygen conditions [5]. Moreover, several studies have implicated a large number of microRNAs in regulating pre- and post-natal chondrogenesis. The goal of this study is to find the regulatory mechanisms of hypoxia-induced microRNAs and its gene targets, which could improve chondrogenesis of stem cells. To achieve this, a meta-analysis of microRNA and mRNAseq of adipose tissue derived (aMSCs) and bone marrow derived (bMSCs) stem cell cultures in hypoxia was completed. Despite that several microRNAs have been described to participate during chondrogenesis of cells, we found hypoxic regulated miR-210 to be essential in the regulation of genes in charge of several functions necessary for cartilage development, chondrogenic differentiation and oxidative stress response. miR-210 also regulates gene expression in concert with the transcription factor HIF1. Several hypoxia responsive genes were regulated in both MSCs. In both MSCs genes that participate in similar biological processes such as hypoxia response, cell metabolism, apoptosis and cell cycle were up-regulated; while genes increased in 
bMSCs were involved in cartilage development and maintenance, and genes increased in aMSCs were involved in bone extracellular matrix synthesis and osteoarthritis development. Remarkably, miR-210 seems to play a role in regulating several cellular processes and functions that could improve chondrogenic differentiation of stem cells for cartilage regeneration therapies. 


\subsection{INTRODUCTION}

Articular cartilage (AC) is a form of hyaline cartilage that is present at the articular surface of bones in the joints. It provides a smooth, lubricated, lowfriction and wear-resistant surface for articulation, translation and rotation between bones. Once damaged, cartilage has a limited capacity to heal and repair. Therefore, preservation of the cartilage tissue is vital for joint health [6]. This preservation depends dominantly on the articular cartilage biochemical and mechanical characteristics. If these characteristics change over time, cartilage degeneration starts [7]. The limited capacity of cartilage to repair damage has led to the development of several cartilage engineering strategies. Cell therapies entail tissue extraction from a healthy donor site and in vitro culture of the cells for several passages until a significant amount of cell is acquired [8]. Slow proliferation rates, extended culture time and dedifferentiation of the cells have been often observed before cells are re-implanted in the patient, leading to poor chondrogenic response and often donor site morbidity. Because healthy chondrocytes reside and survive in a sustained low oxygen environment [8], several authors have hypothesized that hypoxia is an important factor in regulating the growth and survival of chondrocytes [9]. A number of pathophysiological findings propose that a correlation does exist between hypoxia and chondrogenesis [8]. Additionally, several studies have shown that a low oxygen tension environment may be beneficial to preserve the chondrocytic phenotype of the cells during in vitro cultures, avoiding the potential dedifferentiation of the cells during expansion. However, the exact mechanism by which chondrocytes are regulated by oxygen tension remains poorly understood.

During the past decade multiple studies have demonstrated that miRNAs are involved in the hypoxic response and can contribute to the repression of specific genes under low oxygen conditions $[10,11]$. MicroRNAs (miRNAS) are small noncoding RNA molecules of about 20 nucleotides in length and control the 
expression of at least $60 \%$ of protein coding genes. miRNAs participate in posttranscriptional regulation of gene expression, and are involved in RNA silencing. They play a role in the pathogenesis of several diseases including cancer [12]. MiRNAs frequently repress target genes by reducing the half-life time of their mRNA and/or inhibiting protein translation. To do so, miRNAs are incorporated in the RNA-induced silencing complex (RISC) and target the 3' untranslated region (UTR) of mRNAs, leading to translation inhibition or degradation of the mRNA. A single miRNA can regulate hundreds of distinct mRNAS simultaneously and a single mRNA can also be regulated by several distinct miRNAS [13]. In this study we seek to identify oxygen-regulated miRNAs and respective gene targets that regulate cartilage development and maintenance. Therefore, this will provide an adequate mechanism to improve in vitro chondrogenesis of mesenchymal stem cells for cartilage tissue engineering applications.

\subsection{MATERIALS AND METHODS}

\subsubsection{Cell harvest and expansion}

Two different sources of MSCs were used for these studies. Bone marrow stem cells (bMSCs) were derived from bone marrow aspirates approved by the local ethical committee of the Medisch Spectrum Twente. Informed written consent was obtained for all samples. Human bMSCs were isolated from fresh bone marrow samples of three donors (age average: 52 years old, 25\% male, 75\% female) and cultured as described previously by Georgi and colleagues [9]. Adipose derived mesenchymal stem cells (aMSCs) were obtained from three representative donors (age average: 42 years old, 75\% male, 25\% female) that were previously validated for tri-lineage differentiation potential [14]. aMSCs were obtained with approval from the Mayo Clinic Institutional Review Board (IRB) as described by Dudakovic et al. [15]. Both MSCs were expanded in maintenance media. For bMSCs this media consisted of DMEM with $4.5 \mathrm{~g} / \mathrm{L}$ glucose and sodium pyruvate (Corning, Corning NY), supplemented with 10\% 
fetal bovine serum (Atlanta Biologicals, Atlanta, GA) and 1\% penicillin/streptomycin (Gibco/Thermo Fisher Scientific). Maintenance media for aMSCs was composed of Advanced MEM (Gibco/Thermo Fisher Scientific, Waltham, MA) supplemented with 5\% human platelet lysate (PL-Max, MillCreek Life Sciences, Rochester, MN), 1\% penicillin/streptomycin, 1\% Glutamax (Gibco/Thermo Fisher Scientific), and 0.2\% heparin (Baxter, Deerfield, IL). Cells were cultured using standard techniques in $\mathrm{T}-175 \mathrm{~cm}^{2}$ flasks at $37^{\circ} \mathrm{C}, 95 \%$ humidity, and $5 \% \quad \mathrm{CO}_{2}$ until they reached $80 \%$ confluence. Prior to each experiment, cells were detached from T175 flasks by trypsinization using TrypLE Express (Gibco/Thermo Fisher Scientific).

\subsubsection{Cell culture under Normoxia and Hypoxia conditions}

To evaluate miRNA and mRNA expression of both aMSCs and bMSCs in response to oxygen tension, both cells were plated in 6-well plates at a density of 3,000 cells $/ \mathrm{cm}^{2}$ and maintained for 7 days in maintenance media under either normoxic ( $20 \%$ oxygen) or hypoxic $(2.1 \%$ oxygen) conditions using an integrated system with a cell culture hood and an incubator accessible through a gas-lock (IGlove, BioSpherix, New York, NY). After 7 days in culture, total RNA was isolated using the miRNeasy Micro Kit (Qiagen, Hilden, Germany) following the instructions of the manufacturer. Three different biological replicates for each experimental condition (e.g., 3 samples for normoxia and 3 samples for hypoxia) were used concurrently for RNA isolation and subsequent expression analysis to ensure consistency in RNA quality and expression values. RNA concentrations and purity levels were measured using a NanoDrop (Thermo Fisher Scientific).

\subsubsection{RNA extraction from tissues}

Cartilage tissue specimens were collected from patients undergoing tissue removal as part of planned surgical procedures. The specimens used in this investigation were collected under Institutional Review Board (IRB). Written informed consent was obtained for all collected tissues. From the 10 samples 
obtained, 6 samples were collected from 3 different donors from whom we obtained both ankle and knee tissues. Each of the other 4 samples was collected from a different donor. There was an overall predominance of males (90\%) individuals between the ages of 34 and 71 (average was 63 years old). After collection, articular cartilage specimens were classified according to radiological findings using the Collins grading scheme as described previously [16]. For this study, joints were specifically classified using the following criteria: G0 joints displayed normal articular cartilage throughout $(n=5), G 1$ joints had some destruction of superficial cartilage and swelling and/or softening (n=7), G2 joints exhibited extensive superficial damage to articular cartilage but not significant loss of cartilage and no damage to bone $(n=6)$, G3 joints had areas with total articular cartilage loss with observable penetration of defects to the subchondral bone plate $(n=4)$, and a G4 joint was identified as having complete loss of cartilage and full thickness exposure to bone along the articular surface of the joint. Half of the donors were classified as G0 and half as G3/4.

Before RNA extraction, samples were snap frozen in liquid nitrogen and stored at $-80{ }^{\circ} \mathrm{C}$ after surgical harvest. Then, frozen tissues were grounded into a powder using a mortar and pestle and homogenized in Qiazol reagent (Qiagen, Hilden, Germany). Total RNA was extracted using the RNeasy minikit (Qiagen, Hilden, Germany) and quantified using a NanoDrop 2000 spectrophotometer (Thermo Fischer Scientific, Wilmington, Delaware).

\subsubsection{Next generation RNA sequencing}

For samples selected for next generation sequencing, RNA integrity was assessed using the Agilent Bioanalyzer DNA 1000 chip (Invitrogen, Carlsbad, CA). Only samples with an RNA Integrity Number (RIN) and DV200 score greater than our Sequencing Core's minimum cutoff (RIN >6 and DV200 > 50\%) were used for sequencing. RNA sequencing and subsequent bioinformatics analysis were 
performed in collaboration with the Mayo Clinic RNA sequencing and bioinformatics cores as previously described [15].

High-throughput next generation microRNA sequencing was performed using the NEBNext Small RNA library prep kit on an Illumina HiSeq 2000 as described previously by Riester et al. [17]. MicroRNAs sequences were trimmed off adapters using Cutadapt [18] and alignment of these sequences (greater than 17 nucleotides in length) was performed against the reference genome using Bowtie [19]. Then, microRNAs quantification was obtained using miRDeep2 [20]. All other analyses were performed on robustly expressed microRNAs with at least 1 normalized read per million in either of the conditions (e.g. hypoxia or normoxia).

For mRNA sequencing, library preparation was performed using the TruSeq RNA library preparation kit (Illumina, San Diego, CA). Poly-A mRNA was purified using oligo dT magnetic beads. Then, cDNA synthesis was performed on purified mRNA using SuperScript III reverse transcriptase and random primers (Invitrogen). DNA fragments were enriched by PCR using primers included in the Illumina Sample Prep Kit. Sample concentration and size distribution were determined on an Agilent Bioanalyzer DNA 1000 chip and Qubit fluorometry (Invitrogen, Carlsbad, CA). RNA-Seq data was analyzed using the MAPRSeq v.1.2.1 system including alignment with TopHat 2.0.6 [21] and HTSeq software [22]. Expression values for each gene were normalized to 1 million reads and corrected for gene length (reads per kilobase pair per million mapped reads, RPKM).

\subsubsection{Computational analysis and statistics}

Normalized read counts obtained from the RNA sequencing data were analyzed to assess differential gene expression between our different conditions. Soft filtering using RPKM values above 0.1 , fold changes of 1.4 -fold and a $p$-value 
below 0.05 were performed to all our data sets. Statistical analysis was performed using JMP ${ }^{\circledR}$ Pro v10.0.0 (SAS Institute), and $p$ values were determined with two-tailed paired Student T-test.

Computational analysis was achieved using various online software tools. For visualization, Venn diagrams were generated using Venny online tool [23] to compare different conditions. Unsupervised hierarchical clustering was performed using the Pearson correlation method on the MORPHEUS matrix visualization and analysis software [24]. For microRNAs robustly expressed in hypoxia (RPKM>1), a combinatorial target prediction analysis was performed using TargetHub, TargetScan and TargetMiner online software. Gene ontology analysis and functional gene annotation clustering were performed on genes differentially expressed using GO $[25,26]$ and Database for Annotation and Visualization and Integrated Discovery v6.8 database (DAVID 6.8). In addition, gene-gene interaction network and gene function were analyzed using the Search Tool for the Retrieval of Interacting Gene/Protein (STRING) version 10.5 [27] and GeneMANIA 2018 [28].

\subsection{RESULTS}

\subsection{1 microRNAseq analysis reveals a unique hypoxia induced miR- 210 and a clear miRNA footprint of bMSCs on different oxygen conditions.}

To evaluate and identify potential miRNAs that are regulated in hypoxia, three different bMSCs donors were cultured for one week in monolayer cultures and different oxygen environments: normoxia (20\% oxygen) or hypoxia $(2.1 \%$ oxygen). An unsupervised hierarchical clustering using the Pearson correlation method was performed and identified microRNAs that were significantly regulated (RPKM $>1$ and a $p$-value < 0.05). As shown in Figure 4.1.A, the clustering dendogram shows independent clustering of normoxia and hypoxia cultured samples, suggesting a distinctive miRNA response on each oxygen 
condition. No distinction between cell passage 2 (P2) and passage 6 (P6) was observed among the three donors. Fold change between hypoxia and normoxia resulted in 14 hypoxia up-regulated and 2 hypoxia down-regulated microRNAS (Figure 4.1.B).

A

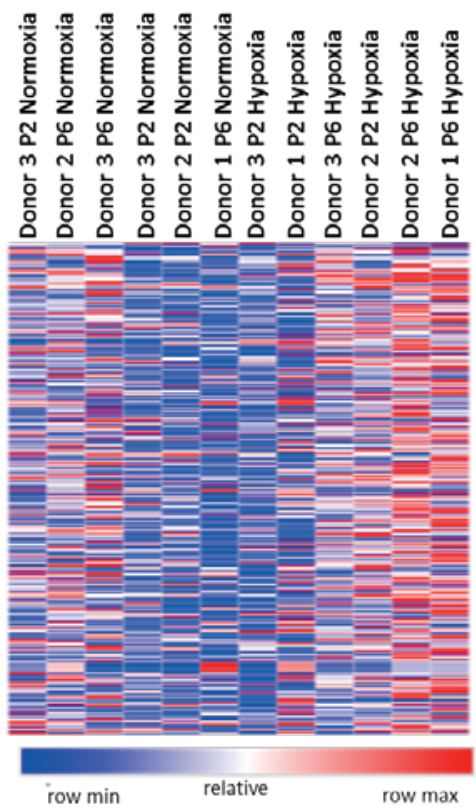

B

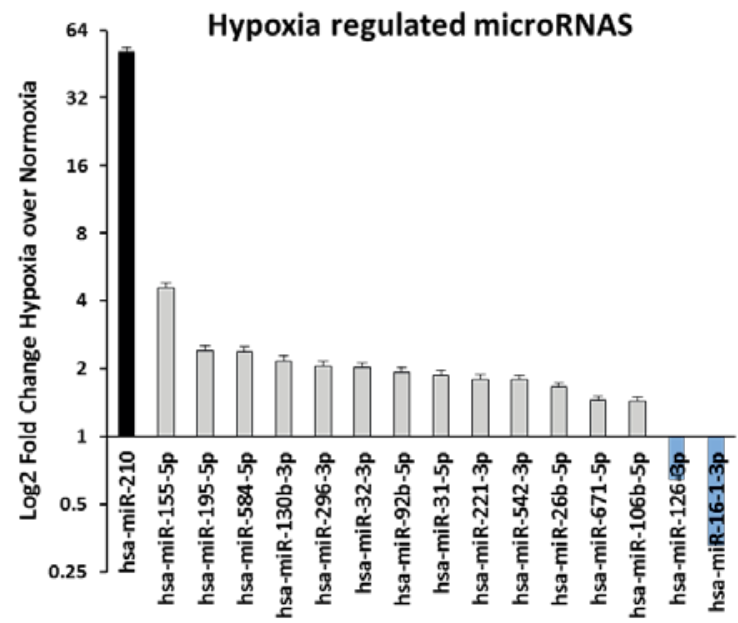

Figure 4.1 MicroRNAseq analysis was performed on three bMSCs donors cultured in normoxia ( $20 \%$ oxygen) and hypoxia (2.1\% oxygen) conditions over one week in monolayer cultures. (A) Unsupervised hierarchical clustering using the Pearson correlation method shows independent clustering of normoxia and hypoxia cultured samples. Passage 2 (P2) and passage 6 (P6) were compared. (B) Fold change between hypoxia and normoxia resulted in 16 regulated miRNAs from which hsa-miR-210 was induced by 50 -fold (black bar). A second group of modestly regulated microRNAS with fold change between 1.4 and 4 was also observed (grey bars). A third group consisted of downregulated miRNAs (blue bars). Expression levels are represented as Log2 of RPKM values between hypoxia and normoxia cultures \pm standard error of the mean. Three biological replicates $(\mathrm{n}=3)$ and one technical replicate were used. 
We observed that hypoxia regulated miRNAs can be distributed over three groups based on their fold change. The first group is represented by hsa-miR-210 which was induced 50-fold in the low oxygen condition (black bar). The second group consists of miRNAs that are only modestly increased (1.4 to 4 fold) in low oxygen conditions (grey bars), which includes hsa-miR-155-5p, hsa-miR-195-5p, hsa-miR-584-5p, hsa-miR-130-3p, hsa-miR-296-3p, hsa-miR-32-3p, hsa-miR92b-5p, hsa-miR-31-5p, hsa-miR-221-3p, hsa-miR-542-3p, hsa-miR-26b-5p, hsamiR-671-5p and hsa-miR-106b-5p. Lastly, 2 miRNAs (hsa-miR-126-3p and hsamiR-16-1-3p) were significantly down-regulated in hypoxia (blue bars). These results proposed miR-210 as the unique hypoxia inducible microRNA.

\subsubsection{Modestly induced miRNAs during low oxygen culture do not participate in a specific molecular program.}

To identify genes that may be regulated by the modestly induced microRNAs in hypoxia, a target prediction analysis was performed. TargetScan software was used on 4 microRNAs (e.g. hsa-miR-221-3p, hsa-miR-155-5p, hsa-miR-26b-5p and hsa-miR-31-5p). More than 2000 genes were predicted targets in any of the 4 hypoxia-controlled miRNAs, whereas only 38 were predicted for all of them. Common targets were represented and compared using a Venn diagram as shown in Figure 4.2.A. 294 genes were targeted by at least 2 miRNAs whereas 32 genes were targeted by 3 or 4 miRNAs. Gene ontology analysis showed there was neither correlation nor interaction between these 32 common genes. In addition, DAVID bioinformatics resources 6.8 demonstrated an absence of specifically enriched pathways.

Then, an unbiased analysis was performed by comparing the 294 predicted targets found on TargetScan against our bMSCs mRNAseq dataset. mRNAseq analysis show only 27 genes were significantly regulated in low oxygen cultures. A comparison of these 27 genes and the 294 miRNA-31/221/155/26b gene targets revealed 11 targets in common (Figure 4.2.B). Log2 fold change between 
hypoxia and normoxia showed only 4 gene targets were significantly regulated. $B D N F$ and PRELP were up regulated more than 1.4 times in hypoxia (black bars) whilst CDKN1C and ZNF704 were down regulated more than 1.4 times (blue bars). Based on our target prediction analysis it is concluded that no overall specific molecular program was targeted by the modestly induced microRNAs in hypoxia.

A
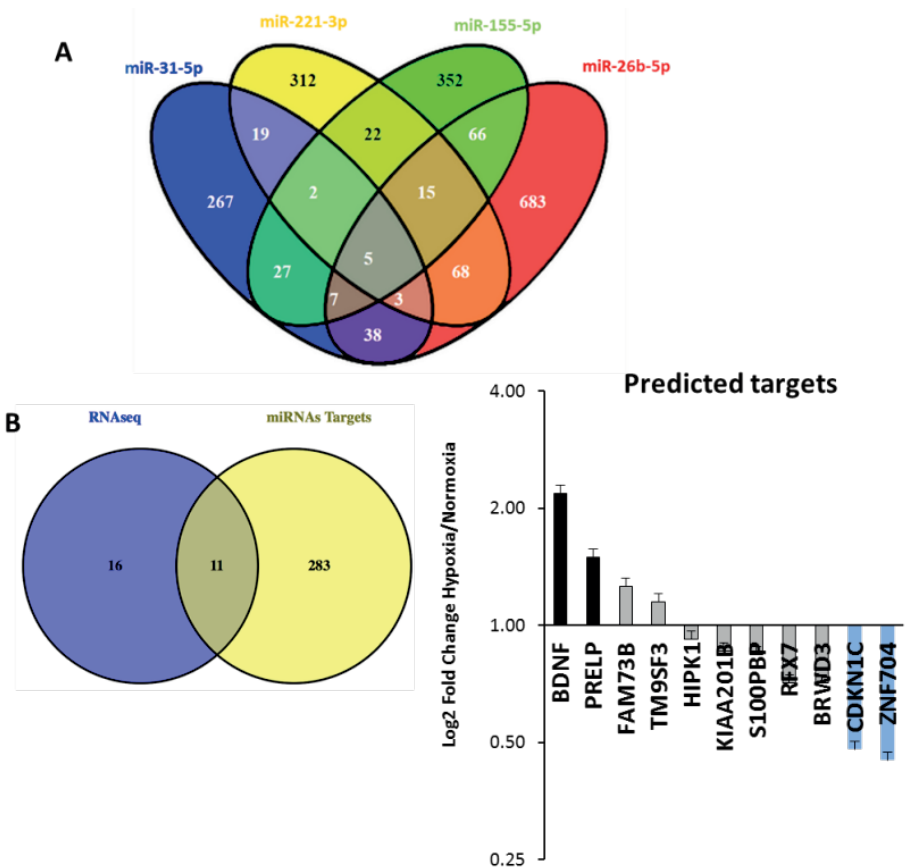

Figure 4.2 TargetScan was used to predict the targets of each of the 4 most abundant modestly induced microRNAs in low oxygen: hsa-miR-221-3p, hsa-miR-155-5p, hsa-miR-26b-5p and hsamiR-31-5p. (A) Although, more than 2000 genes were predicted targets of any of the 4 hypoxiacontrolled microRNAs, only 294 genes were targeted by at least 2 of them. (B) Unbiased analysis of all targets was performed by comparing the 294 gene targets found on TargetScan against the 27 significantly regulated genes from our bMSCs mRNAseq dataset. Log2 fold change between hypoxia and normoxia showed only 4 from the 11 genes in common were significantly regulated in hypoxia: $B D N F$ and PRELP (1.4 fold change in hypoxia, black bars) and CDKN1C and ZNF704 (0.7 fold change in hypoxia, blue bars). Expression levels are represented as Log2 of RPKM values between hypoxia and normoxia cultures \pm standard error of the mean. Three biological replicates $(n=3)$ and one technical replicate were used. 


\subsubsection{Hypoxia regulated miR-210 gene targets are mostly involved in stress response but also in cartilage regulatory programs.}

Similarly, a target prediction analysis was performed on miR-210 using three different algorithms: TargetHub, TargetScan and TargetMiner. The analysis indicated 1210 genes that were predicted using TargetHub, 4099 using TargetScan and 4525 using TargetMiner. Venn diagram comparison using Venny revealed a total of 2429 genes that were predicted by at least two algorithms (Figure 4.3.A) and these genes were considered potential miR-210 targets. From these, a total of 182 genes were predicted by three algorithms and were considered bona fide target genes of miR-210.

An integrated analysis of miR-210 potential and bona fide targets and bMSCs mRNAseq was performed to expose miR-210 gene targets regulated in hypoxia. First, a comparison between the 2429 potential miR-210 gene targets and the mRNAseq dataset was done revealing 191 down-regulated genes in hypoxia and 294 up-regulated. Since miRNAs function is to down-regulate target genes, further analysis was performed only in down-regulated genes. Gene ontology analysis was completed and exhibited involvement of these genes in several cellular processes and functions. Among the cellular functions we observed cellular response to chemical stimulus ( $\mathrm{n}=8, p$-value 0.0327$)$, positive regulation of signal transduction ( $\mathrm{n}=7, p$-value 0.0285$)$, positive regulation of cell signaling $(\mathrm{n}=7, p$-value 0.0276$)$, positive regulation of cell communication $(\mathrm{n}=7, p$-value 0.0151), positive regulation of cell differentiation ( $n=6, p$-value 0.0138), regulation of signaling receptor activity $(n=5, p$-value 0.0334$)$ and skeletal system development ( $\mathrm{n}=5$, $p$-value 0.0147) (Figure 4.3.B). Molecular function regulation $(\mathrm{n}=7, p$-value 0.0142$)$, receptor regulator activity $(\mathrm{n}=5, p$-value $0.00701)$ and receptor ligand activity $(n=5, p$-value 0.00431$)$ are among the cellular functions found (Figure 4.3.C). Results were shown as fold enrichment, number of genes ( $\mathrm{n}=\#$ of genes) and Bonferroni-corrected for $\mathrm{P}<0.05$. Moreover, five different pathways were found for these genes: apoptosis (BCL2), hypoxia 
inducible pathway (EPO), cell cycle regulation (HOXB9, IGF1 and $S F N$ ), TGF $\beta$ signaling pathway $(B M P 8 B)$ and WNT signaling pathway (ENT11, WNT7B and WNT9A). Therefore, miR-210 potential gene targets participate in cell differentiation, skeletal system development and cellular stress response.

A

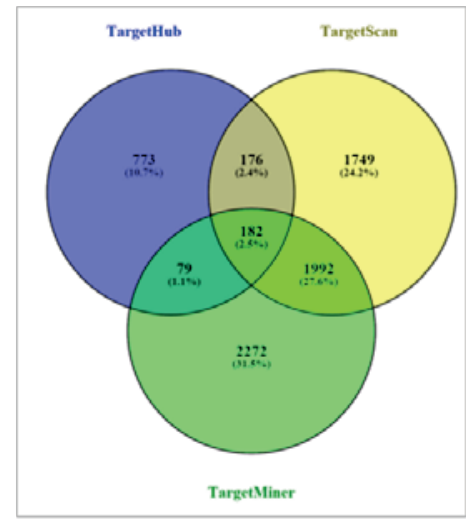

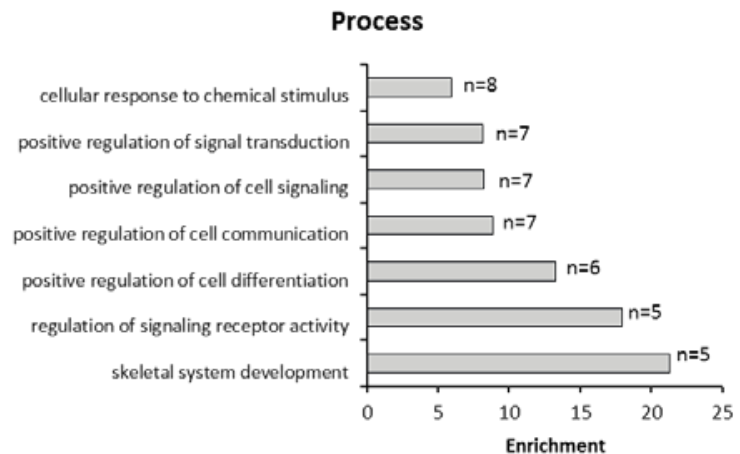

C

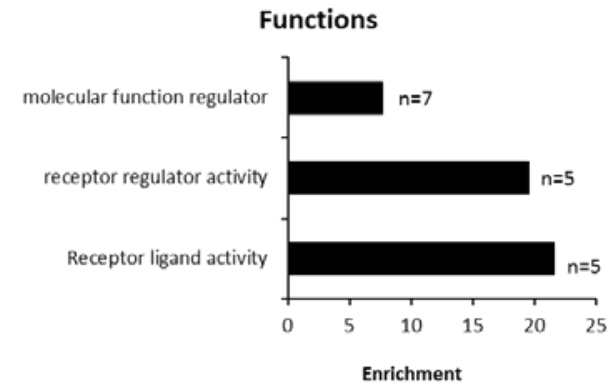

Figure 4.3. A target prediction analysis was performed to investigate miR-210 targets that are involved in the response of bMSCs to low oxygen. (A) Venn diagram comparison revealed a total of 2429 genes that were predicted by at least two algorithms whereas 182 genes were predicted by three algorithms. (B-C) Gene ontology analysis exposed participation of potential miR-210 gene targets down-regulated in hypoxia in different cellular processes (B) and different functions (C).

Further analysis was performed on the 182 miR-210 bona fide gene targets. A comparison against the down-regulated genes during the hypoxic response in the bMSCs mRNAseq data was completed. The comparison between these 103 hypoxia down-regulated genes and the 182 miR-210 predicted targets showed 5 genes in common: CENPP, BACH2, PELI2, WISP1 and WNT11. Gene functional analysis revealed centromere protein $\mathrm{P}$ (CENPP) is involved in cell cycle, BTB and 
CNC homology 1 basic leucine zipper transcription factor 2 (BACH2) is linked to oxidative stress, pellino E3 ubiquitin protein ligase family member 2 (PELI2) participates in post-transcriptional control, and WNT1 inducible signaling pathway protein 1 (WISP1) and wingless-type MMTV integration site family member 11 (WNT11) are involved in the WNT signaling pathway. Gene interaction analysis performed using GeneMania presented co-expression of gene targets WISP1, BACH2 and CENPP (purple line) (Figure 4.4.A). A tight interaction between WISP1 and WNT11 was observed. Both genes participate closely in the WNT signaling pathway and interact with several molecules within the pathway.

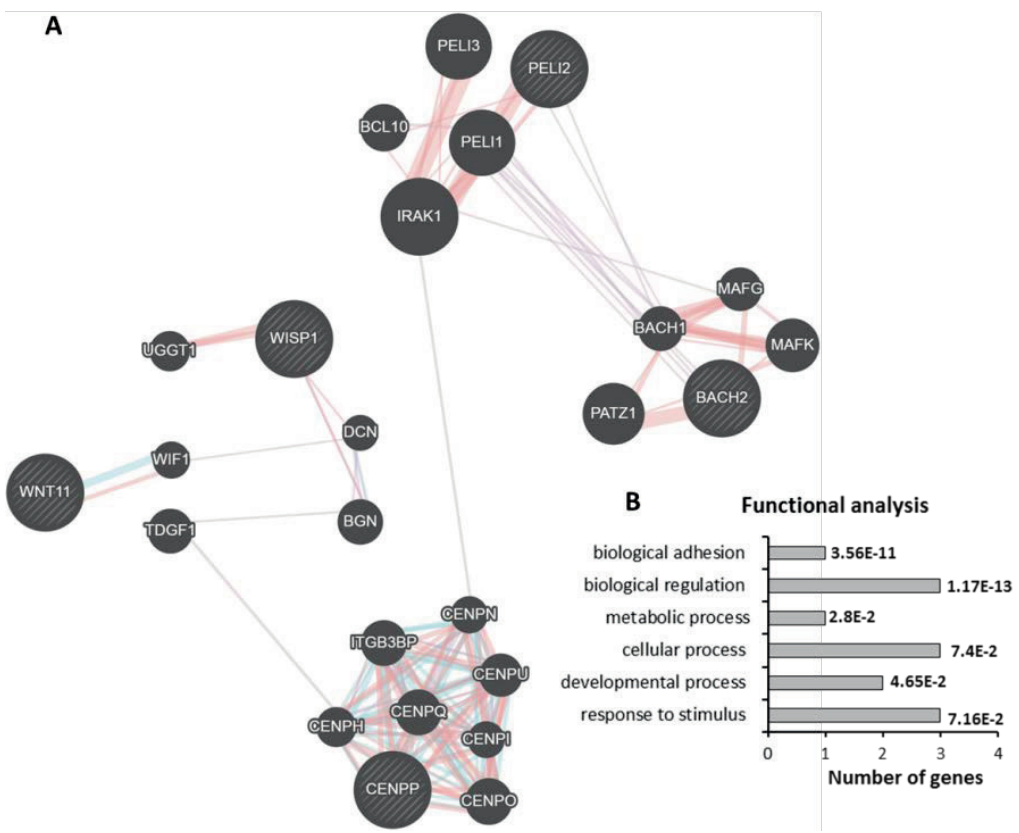

Figure 4.4 Identification of genes that can be modulated by miR-210 expression during the hypoxic response was completed. (A) 5 genes were identified: CENPP, BACH2, PELI2, WISP1 and WNT11. Gene interaction analysis shows co-expression of WISP1, BACH2 and CENPP (purple line). Physical interaction of the genes is shown with the red lines. Three different pathways were observed: WNT and hedgehog signaling pathways, and NF-KappaB pathway Toll-like receptor signaling pathway

(blue lines). (B) Bar graph represents functional analysis of genes that interact with hypoxic responsive miR-210 genes with its respective $p$-value. 
Physical interactions of the genes are represented with red lines. Moreover, three different pathways were observed with this analysis (blue lines). WNT11 participates in the WNT and hedgehog signaling pathways, PELI2 participates in the NF-KappaB pathway Toll-like receptor-signaling pathway whereas CENPP was found to interact in cell cycle and mitosis of the cells. Gene functional analysis revealed these genes are involved in cellular response $(\mathrm{n}=3, p$-value 0.0716), developmental processes ( $\mathrm{n}=2, p$-value 0.0465$)$, metabolism $(\mathrm{n}=1, p$ value 0.028$)$, cell adhesion $(\mathrm{n}=1, p$-value $3.56 \mathrm{E}-11)$ and regulation $(\mathrm{n}=3, p$-value 1.17E-13) (Figure 4.4.B).

Functional protein association network analysis (STRING) depicted an interaction of WNT11 with several other factors of the WNT/Frizzled signaling pathway such as WIF1, FZD4, FZD2, SFRP1, SFRP2 and FZD6 (Figure 4.5.A). STRING network analysis shows a correlation between WISP1 and several proteoglycan proteins decorin (DCN) and byglican (BGN) while it interacts with metalloproteinases MMP2 and MMP13 (Figure 4.5.B). It also revealed an interaction of BACH2 with PAX and MAF genes, which are involved in oxidative stress response (Figure 4.5.C). CENPP was found to participate in assembly of kinetochore proteins and mitotic progression (Figure 4.5.D and E) whereas PELI2 is involved in lipopolysaccharide signalling.

To summarize our findings, we observed that miR-210 under low oxygen tension targets specific genes that are involved in cartilage development and oxidative stress response. 


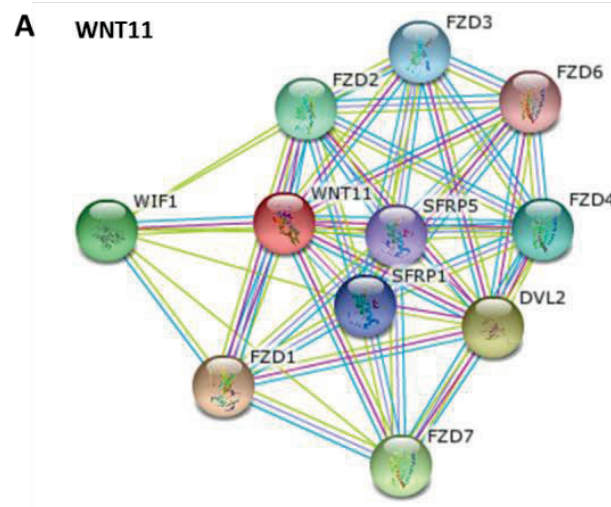

C BACH2

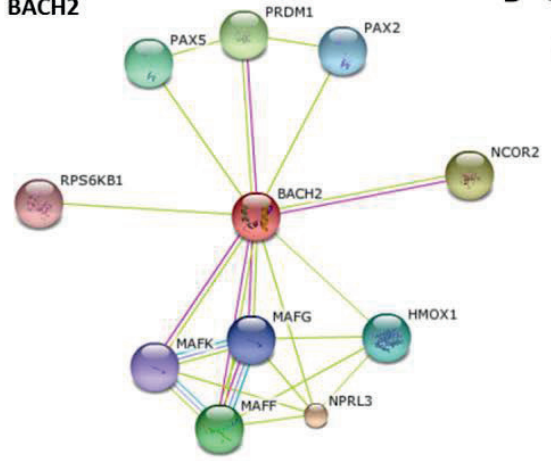

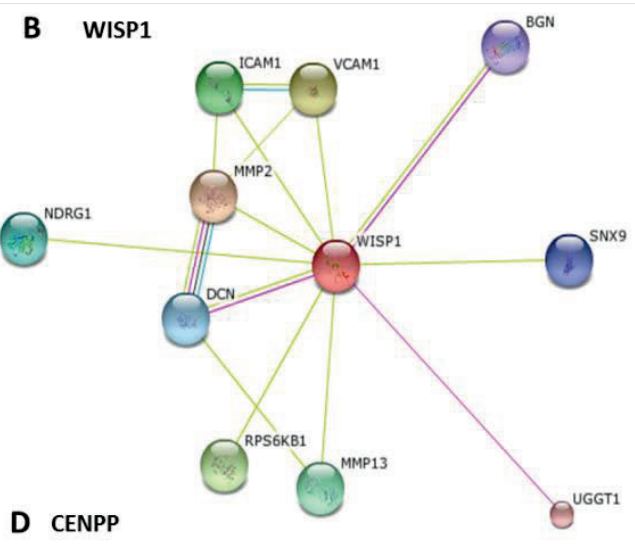

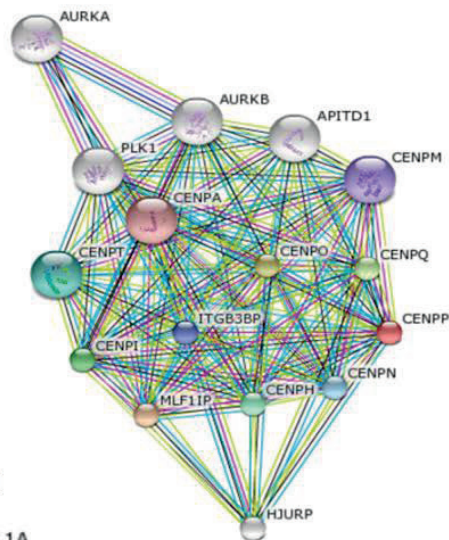

E PELI2

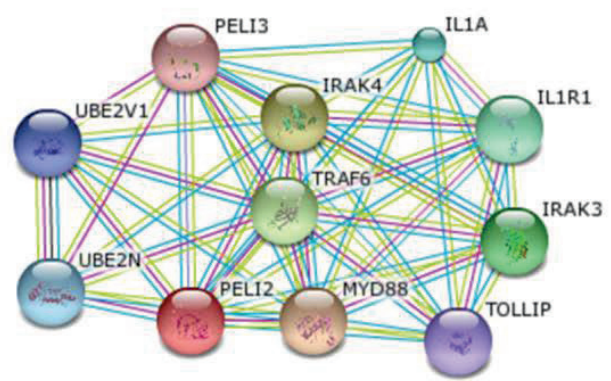

Figure 4.5 (A) STRING network analysis revealed interaction of WNT11 with several other factors of the Wnt/Frizzled signaling pathway. A correlation between WISP1 and decorin (DCN), byglican

(BGN), MMP2 and MMP13 was observed. (B) In contrast, BACH2 was linked to coordinating transcription activation and repression, whereas CENPP and PELI2 (C) have neither interaction nor relation with cartilage development or oxidative stress proteins. 


\subsubsection{Common gene targets of hypoxia inducible factor HIF1 and miRNA-210 in bMSCs play essential roles in tissue development, oxidative stress response and cell cycle regulation.}

We next complemented the list of potential miR-210 targets with a literature survey. Table 4.1 describes 86 genes identified as miR-210 targets in the literature and their functional classification. The genes are involved in various cellular processes such as: cell cycle, apoptosis, angiogenesis, DNA damage, mitochondrial metabolism, development and differentiation, DNA binding, chromosome structure, membrane trafficking, migration and adhesion, translation and transcription, and protein modification and transport [44-54]. Interestingly, the majority of these genes participate in cell cycle regulation for growth survival, and development and differentiation during adipogenesis and osteogenesis. From those 86 genes only 56 have been experimentally validated.

\begin{tabular}{|c|c|c|c|c|c|c|c|c|c|c|c|}
\hline Cell cycle & Angiogenesis & Apoptosis & $\begin{array}{c}\text { DNA } \\
\text { damage }\end{array}$ & $\begin{array}{l}\text { Witochond rial } \\
\text { Eetabolisa }\end{array}$ & $\begin{array}{l}\text { Develope ent } \\
\text { Differentiation }\end{array}$ & DinA & $\begin{array}{l}\text { Chromosome } \\
\text { structured } \\
\text { Inactivation }\end{array}$ & $\begin{array}{l}\text { Meebrane } \\
\text { Trafficking }\end{array}$ & $\begin{array}{l}\text { Mgration } \\
\text { Adhesion }\end{array}$ & $\begin{array}{l}\text { Translationd } \\
\text { Transeription }\end{array}$ & $\begin{array}{c}\text { Profein } \\
\text { Dodfication } \\
\text { Transport }\end{array}$ \\
\hline 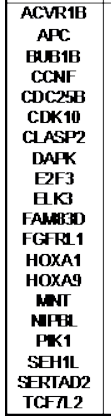 & $\begin{array}{c}\text { EFNA3 } \\
\text { BK3 } \\
\text { PIP1B }\end{array}$ & $\begin{array}{c}\text { AFM3 } \\
\text { BC12 } \\
\text { CASPBAP2 } \\
\text { DAPK } \\
\text { ERF3 } \\
\text { MNI } \\
\text { PIP1B } \\
\text { RAD52 } \\
\text { UEOAN1 }\end{array}$ & RAD52 & $\begin{array}{c}\text { AIP11C } \\
\text { COX 10 } \\
\text { DOAH1 } \\
\text { EK3 } \\
\text { GPD1L } \\
\text { HYP = SEID2 } \\
\text { ISCU1 } \\
\text { ISCU2 } \\
\text { NDUFA4 } \\
\text { SDHD1 }\end{array}$ & $\begin{array}{c}\text { ACVR1B } \\
\text { APC } \\
\text { HONF } \\
\text { CTGF } \\
\text { GNA3 } \\
\text { EK3 } \\
\text { HECTD1 } \\
\text { HOXA1 } \\
\text { HOXA3 } \\
\text { HOXA9 } \\
\text { NB1 } \\
\text { MNI } \\
\text { NFEA } \\
\text { NPTX1 } \\
\text { TCFA2 }\end{array}$ & $\begin{array}{l}\text { CAX1 } \\
\text { CHD9 } \\
\text { CFB2 } \\
\text { RAD52 }\end{array}$ & $\begin{array}{c}\text { CEX1 } \\
\text { CHD9 } \\
\text { NPE } \\
\text { suCHD1 } \\
\text { XIST }\end{array}$ & $\begin{array}{l}\text { ABCB99 } \\
\text { TNPO1 }\end{array}$ & $\begin{array}{c}\text { APC } \\
\text { CLASP2 } \\
\text { EFM3 } \\
\text { HOXA3 } \\
\text { MDGA1 } \\
\text { MA1P1 } \\
\text { NCAM1 } \\
\text { PAHB } \\
\text { PIPN1 }\end{array}$ & $\begin{array}{c}\text { CHF2 } \\
\text { CFEQ } \\
\text { HMFB } \\
\text { KCMF1 } \\
\text { UPOIN1 }\end{array}$ & $\begin{array}{l}\text { PIAR1 } \\
\text { TNPO1 }\end{array}$ \\
\hline
\end{tabular}

Note: Genes listed in this table were summarized from references 44 to 54 .

Literature review was also performed in order to obtain a list of hypoxia inducible factor 1 (HIF1) targets. Around 233 of them have been described in the literature [55-57] and are shown in Table 4.2. These genes participate in several molecular programs such as oxygen delivery, cell survival, glucose and energy metabolism, cell migration and adhesion, cell proliferation and viability, angiogenesis, apoptosis and gene transcription [55-59]. GO analyses on HIF1 
targets revealed different functions including blood vessel development, positive regulation of MAP kinase activity, cell-cell signaling, positive regulation of muscle contraction, regulation of cell development, apoptosis regulation and most importantly genes involved in skeletal system development (TIPARP, EDN, PTGS2), oxidative stress response (STC2).

Since miR-210 is the only miRNA that is robustly regulated in hypoxic cultures of bMSCs we examined a potential overlap and/or co-regulation of gene expression between miR-210 and HIF1. Comparison showed that 71 of the 233 genes regulated by HIF1 (27\%) are also potential target genes of miR-210. STRING network and GO analysis was performed on the common genes. Interestingly, several functions and pathways were revealed: bone remodeling (FOSL2), hypoxia inducible factors (EPO), cell cycle arrest and apoptosis (FOXO3, EIF2S1 and PIK3R3), inhibition of matrix metalloproteinases (MMP14) and cytokines and inflammatory response (PDGFA, BBID, LEP and EPO).

A comparison of these common targets and our mRNAseq data shows 7 HIF1/miR-210 common targets were found up-regulated in hypoxia, whilst 1 gene was found down-regulated. The hypoxic up-regulated genes included STC2, IGF2, CHAC1 and CDC42EP4. GO analysis was performed on these genes which revealed participation in several functions including apoptosis (CHAC1), response to endoplasmic reticulum stress (CHAC1), Notch signaling pathway (CHAC1), skeletal system development (IGF2), response to oxidative stress and hypoxia (STC2) and organization of actin cytoskeleton (CDC42EP4). In contrast, the down-regulated gene PIM1 has been described to participate in apoptosis and cell cycle regulation. Thus, hypoxia inducible factor and miR-210 regulated genes share common functionality. They both participate in the regulation of genes essential for oxidative stress response and cell cycle and tissue development. For further experimental analyses, Chapter $\mathbf{5}$ will focus on hypoxia down-regulated target genes with a role in tissue development and oxygen response. 


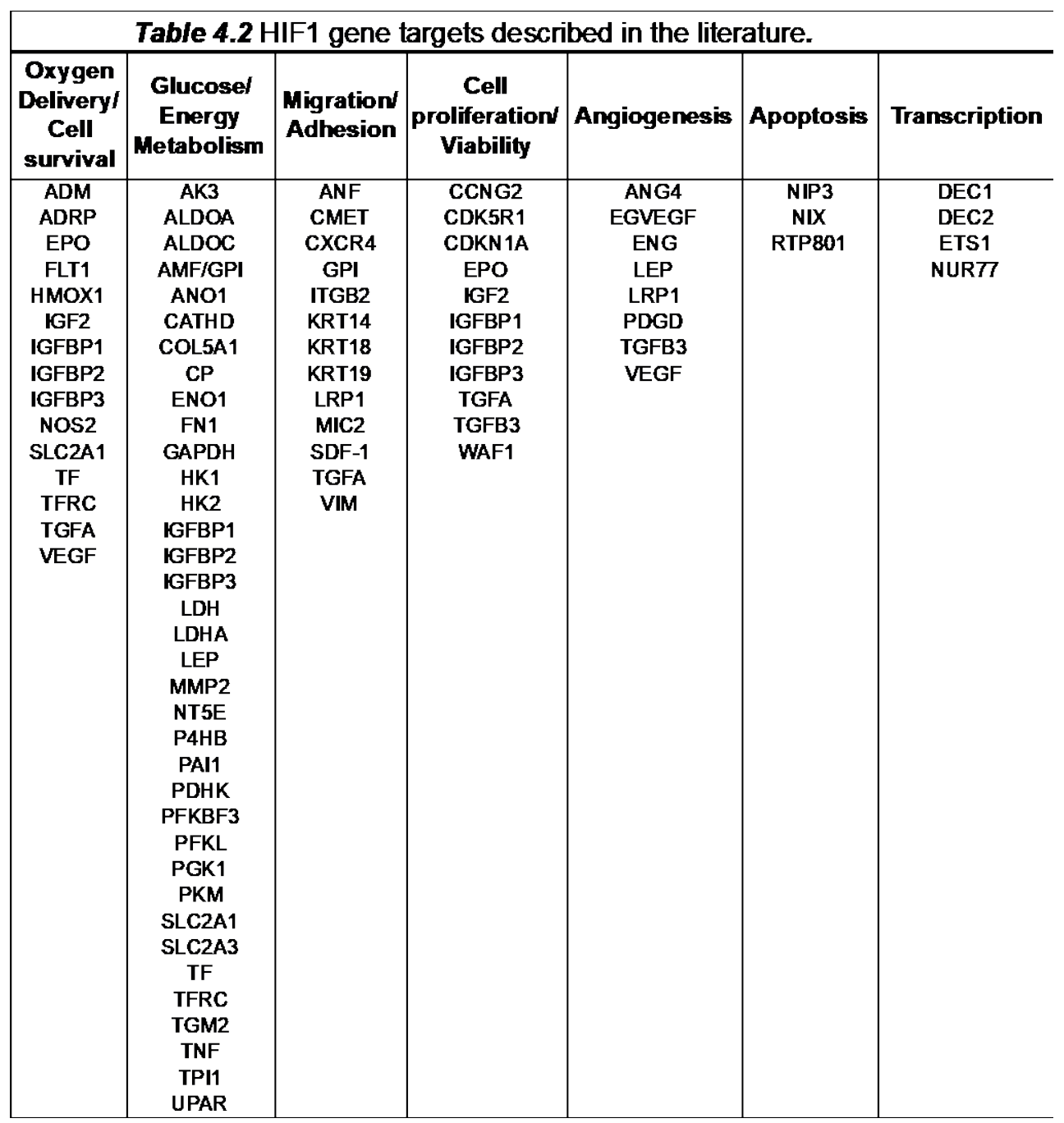

Note: Genes listed in this table were summarized from references 55 to 59.

\subsubsection{Targets genes of hypoxia inducible factor HIF1 and microRNA-} 210 in aMSCs play essential roles in oxidative stress response, tissue homeostasis and inflammation.

To test whether our observations in bMSCs can be extrapolated to other mesenchymal stem cell sources, a microRNAseq was performed on aMSCs cultured in monolayer during 14 days under hypoxia or normoxia conditions. Analysis using a soft filter of fold change (hypoxia/normoxia) above 1.4 and a $p$ - 
value below 0.05 (paired, T-test) exhibited 13 up-regulated miRNAs and 182 down-regulated miRNAs in low oxygen cultures. For our analyses we only focused on microRNAs increased in hypoxia. Among the 13 up-regulated miRNAs, the most abundant were miR-210 with more than 25 -fold change and miR-151a-3p, miR-423-5p, miR-181a-2-3p, miR-195-3p all increased by 1.4 to 5 fold (Figure 4.6.A). Comparison of all miRNAs regulated in both MSCs showed that miR-210 was the only up-regulated miRNA in common whereas miR-378-a$3 p$ was the only miRNA down-regulated in both cells. Hence, in both bMSCs and aMSCs miR-210 was the only strongly up-regulated miRNA under hypoxic conditions.

To identify potential miR-210 targets that regulate the hypoxic response in aMSCs, an mRNAseq analysis was performed. Soft filtering of FC>1.4, RPKM $>0.01$ and $p$-value $<0.05$ (paired, t-test) was applied to all dataset values. Comparison against the $182 \mathrm{miR}-210$ genes predicted during target prediction analysis (shown on Figure 4.3) revealed only 15 potential miR-210 targets regulated in aMSCs under low oxygen tension. 2 miR-210 gene targets were found significantly increased in hypoxia (e.g. EFNA3 and CELF2) whereas 13 targets were down-regulated (e.g. FGFRL1, CDC25B, CCNF, BUB1B, FAM83D, SEH1L, CLASP2, CASP8AP2, COX10, GPD1L, TNPO1, SMCHD1 and PTPN1) (Figure 4.6.B). A cell type specific regulation was observed for FGFRL1; in bMSCs this gene was down-regulated whereas it increased 1.67 times in aMSCs.

STRING gene/gene interaction analysis showed co-expression of several of these hypoxia-regulated genes and co-expression with genes involved in cell cycle such as CCNB2, CCNA, CENPE and MKI67 (purple line). Involvement in Cadherin and Heme signaling pathways by most genes is represented with blue lines (Figure 4.7.A). GO analysis shows up-regulated genes CELF2 and EFNA3 are related to development of skeletal muscle and, vascular and epithelial development. In 
contrast, GO analysis on down-regulated genes revealed 2 of these genes have been previously implicated in oxygen response and musculoskeletal development (e.g. FGFRL1 and COX10).
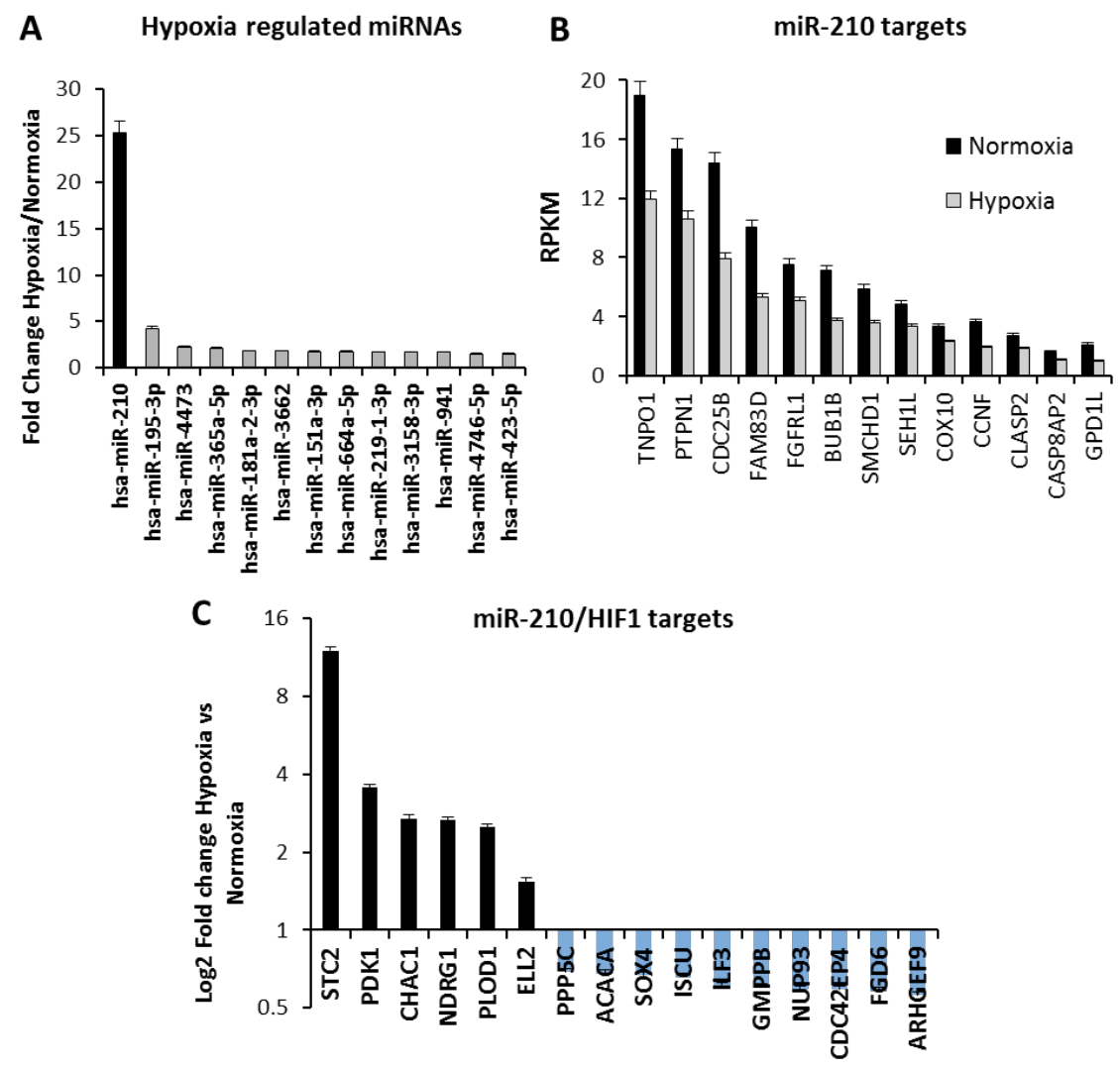

Figure 4.6 MicroRNA and mRNA combinatorial analysis were performed on aMSCs. (A) Most abundant microRNAs up-regulated in aMSCs. Expression levels are represented as fold change of RPKM values \pm standard error of the mean. (B) 15 miR-210 gene targets regulated in hypoxia: 2 up-regulated genes while 13 were down-regulated in hypoxia. Expression levels are represented as mean RPKM value \pm standard error of the mean. (C) Log2 fold change of miR210/HIF1 common targets regulated in hypoxic aMSCs. 6 common gene targets were found up-regulated in hypoxia while 10 targets decreased. Expression levels are represented as Log2 of RPKM values between hypoxia and normoxia cultures \pm standard error of the mean. Three biological replicates $(n=3)$ and one technical replicate were used. 
GO classification was represented in a pie chart showing that $35 \%$ of regulated genes are involved in cellular processes, $28 \%$ in cell metabolism, $13 \%$ in biological regulation, $8 \%$ in cellular organization and biogenesis whereas a small percent is involved in localization, reproduction and stimulus response (Figure 4.7.B).

Additionally, identification of HIF1/miR-210 common targets regulated during aMSCs hypoxic response was completed. As shown in Figure 4.6.C, 6 common gene targets were found up-regulated in hypoxia (e.g STC2, PDK1, CHAC1, NDRG1, PLOD1 and ELL2). Remarkably, oxidative stress responsive gene STC2 was significantly up-regulated in both MSCs. GO analysis revealed involvement of these genes in functions including hypoxia response (PLOD1, NDRG1, and PDK1), response to endoplasmic reticulum stress (CHAC1), oxidative stress response (STC2) and regulation of transcription (ELL2). In contrast, 10 gene targets were decreased in hypoxia (e.g. ISCU, PPP5C, SOX4, CDC42EP4, ARHGEF9, ACACA, FGD6, ILF3, GMPPB and NUP93). These genes are involved in regulation of apoptosis (ARHGEF9), tissue homeostasis (ACACA, FGF6 and ISCU), response to hydrogen peroxide (NUP93 and PPP5C), gene expression (ILF3), organization of actin cytoskeleton (CDC42EP4) and skeletal tissue development (SOX4). Participation in 4 different signaling pathways, HIF1A signaling pathway (PDK1), Notch signaling pathway (CHAC1), SMAD signaling pathway (NUP93) and MAPK signaling pathway $(P P P 5 C)$ was observed.

Furthermore, target prediction analysis was performed for the 4 most abundant modestly induced miRNAs (e.g. miR-151a-3p, miR-195-3p, miR-493-5p and miR181a-2a-3p) in aMSCs. 12 gene targets were predicted for all 4, SOCS5, RYBP, ATP2A2, PKIA, GABRB1, AGO2, ZFAND5, PURB, ZNF704, TANC2, RPS6KA3 and FTO. PANTHER pathway analysis on the genes indicated several pathways related to these genes, including CCKR signaling, inflammation mediated by chemokines, 
cytokines signaling pathway, insulin and IGF activated pathway, interferon gamma signaling pathway, interleukin signaling, PDGF signaling and RAS pathway.

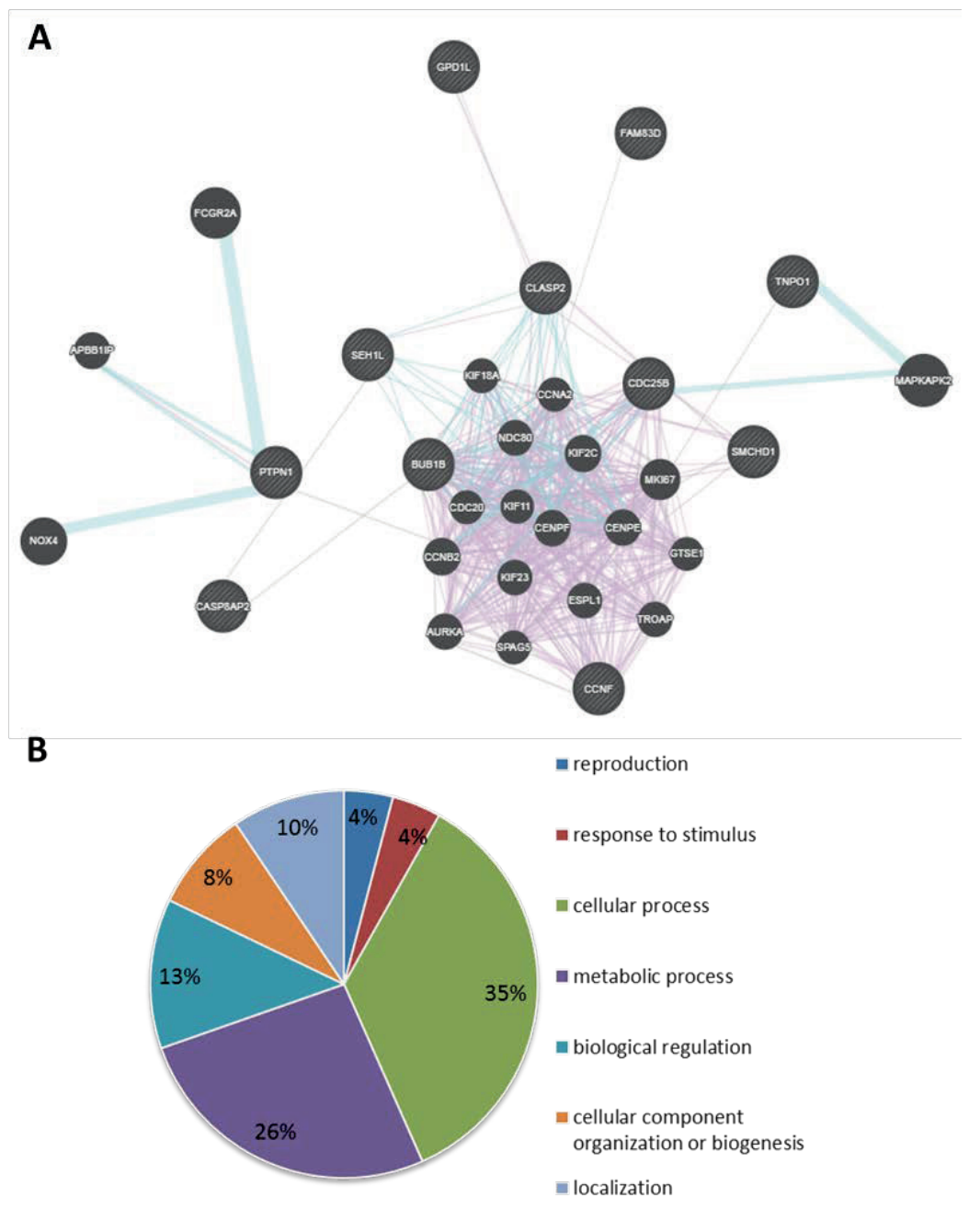

Figure 4.7. (A) STRING gene/gene interactions analysis showed co-expression of several of hypoxia-regulated genes. (B) GO classification was represented in a pie chart. 
In summary, miR-210 targets regulated in hypoxic aMSCs are involved in musculoskeletal tissue development and stress response of the cells to low oxygen whereas miR-210/HIF1 targets are involved mostly in stress response and tissue homeostasis. Likewise, modestly induced miRNAs seem to participate in the inflammatory response of the cells to low oxygen.

\subsubsection{MiR-210 may play an important role in the inflammatory response and cartilage homeostasis in healthy cartilage tissues.}

To categorize mRNAs that are regulated in healthy cartilage, mRNAseq analysis was performed on healthy (no OA) and diseased cartilage (G3/4). The top 20 of genes up-regulated in G0 vs G3-G4 cartilage (Fold change G0/G3-G4 >1.4 and $p$ value below 0.05) are shown in Figure 4.8.A. GO analysis showed most of the genes were involved in skeletal system development, ECM organization and cartilage development. Among their different functions we found TGF $\beta$ production, glycosaminoglycan metabolic process, chondroitin sulfate biosynthetic process, catabolic process, and wound healing. Moreover, some of these genes (e.g. TNFRSF11B, FRZB, LUM, CTGF and DCN) were also involved in various signaling pathways including $\mathrm{TNF} \alpha, \mathrm{WNT}, \mathrm{TGF} \beta$ and VEGF. Interestingly, genes involved in anoxia, inflammatory response, oxygen homeostasis and cellular response to hypoxia such as CTGF, CDO1, EPAS1/HIF2A and HIF1A were also up-regulated in healthy cartilage compared to diseased tissues. Some other genes regulated in healthy cartilage included ZFP36, ID2 and SLC38A2, which are linked to cellular response to stimulus, regulation of metabolic process and cellular response to mechanical stimulus.

Figure 4.8.B displays miR-210 expression during osteoarthritis progression. MiR-210 expression values increased in the first stage of osteoarthritis (G1 stage) by 104.9 -fold ( $p$-value $=0.032$ ) compared to G0 while increasing 21 to 8fold on chronic stages G2 ( $p$-value $=0.045)$ and G3/4 ( $p$-value=0.048) respectively, compared to healthy cartilage (no OA, G0). This result suggests a 
potential role of miR-210 in early osteoarthritis while its importance may decrease during the progression of disease.

A

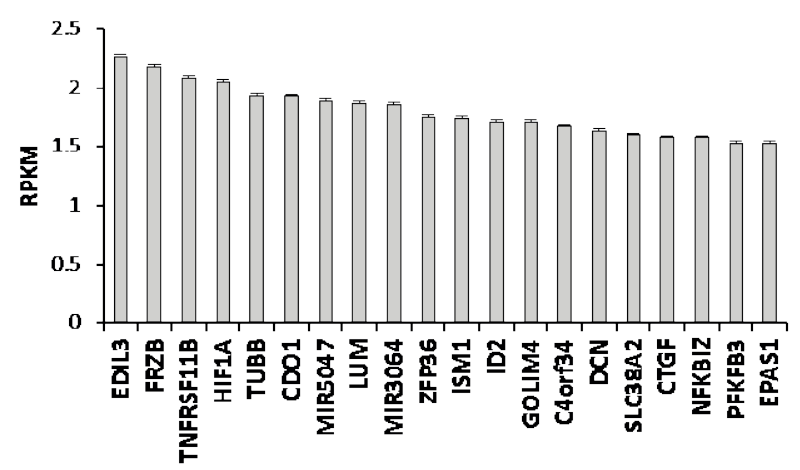

B

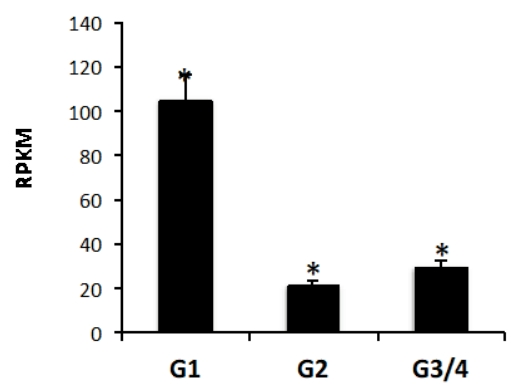

Figure 4.8 mRNAs that are regulated in healthy cartilage (no OA, G0) against diseased cartilage (G3/4). (A) Top 20 of up-regulated genes in healthy cartilage. (B) MiR-210 RPKM values during osteoarthritis progression. Expression levels are represented as RPKM values \pm standard error of the mean. Three biological replicates $(n=3)$ and one technical replicate were used. Statistical analysis is represented as * for $p<0.05$.

To define miR-210 potential gene targets in healthy cartilage (G0), an analysis of genes regulated in healthy cartilage (G0, no $\mathrm{OA})$, genes regulated in severe $\mathrm{OA}$ cartilage (G3-4) and miR-210 predicted targets was performed. 764 genes were up regulated whilst 247 genes were down regulated in healthy cartilage (no OA, G0) when compared to severe OA samples (G3-4). From those, 80 miR-210 gene targets were up regulated in healthy cartilage (no OA). Among these genes: FRZB, PRKCE, PRKCQ, EP300 and ACVR1B, have been previously associated to cartilage development related pathways such as WNT and BMP signaling pathways. The list of the 80 genes is listed in Table 4.3A. From the 243 genes down-regulated in G0 vs G3-G4 cartilage, 29 genes were also miR-210 targets and are listed in Table 4.3.B. Functional analysis showed some of these genes are involved in cell response during hypoxia (e.g. STC2) and cartilage degradation (e.g. MMP14 and 
ADAMTS10). Hence, healthy (no OA, G0) cartilage presents specific gene expression of genes involved in cartilage homeostasis and cartilage development.

Table 4.3 List of regulated genes in healthy cartilage (no OA, G0) when compared to diseased cartilage (severe OA, G3-4) that are also miR-210 targets.

A

\begin{tabular}{|cccccc|}
\hline \multicolumn{7}{|c|}{ Up-regulated mi-R210 targets } \\
\hline FR2B & WHSC1L1 & FITM2 & MFHAS1 & PRKCE & GRAMD1C \\
TMOD1 & EP300 & VPS13D & ESR1 & ZDBF2 & NLRC5 \\
FOSL2 & ASH1L & PRKACB & PRKCQ & MKL2 & PHF16 \\
TMTC1 & RAPH1 & MFAP3L & CLOCK & MOCOS & GRAMD2 \\
ANTXR1 & ZNF385B & SLC30A2 & ATP11A & KLF12 & KCND3 \\
B4GALT5 & TMEM133 & NCAPD3 & TMED8 & RALGPS1 & TANC2 \\
HIP1 & TUBGCP6 & ADARB1 & HMBOX1 & EMILIN3 & GABRB1 \\
CLIP2 & MLL2 & FOXI2 & SLC37A1 & RIC3 & HIST1H2AK \\
MGLL & CDC42BPA & EGFR & HS3ST3B1 & PPM1L & SAMD12 \\
CACNA1C & MYO18A & ACVR1B & BAZ2B & GABRR2 & TIMD4 \\
SLC12A7 & RAPGEF5 & SETBP1 & EPB41L5 & STC1 & PRLR \\
ACACA & PTPRD & HDAC4 & SPEG & PAIP2B & FSD1L \\
CDK18 & TEAD3 & DNAJC18 & TET2 & PDE3B & MDGA1 \\
CLLU1 & FGF17 & & & & \\
\hline
\end{tabular}

B

\begin{tabular}{|cccccc}
\hline \multicolumn{7}{c}{ Down-regulated miR-210 targets } \\
\hline LAMC2 & TMEM45A & NDUFB5 & CLSTN1 & PDLIM7 & COL6A1 \\
SPATA20 & ADAMTS10 & SPNS1 & SNX8 & TMEM101 & COTL1 \\
PKP1 & CSMD2 & QSOX1 & PGAP3 & STC2 & PHLDA3 \\
HSPA5 & PLCXD1 & HM13 & STK24 & MMP14 & NT5M \\
SLC16A3 & COX6B2 & EHD2 & RASA3 & TCN2 & \\
\hline
\end{tabular}

\subsubsection{Similar regulatory mechanisms enhance chondrogenic differentiation of MSCs during the hypoxic response.}

To define the microRNAs and gene targets involved in the regulation and response of MSCs (both bMSCs and aMSCs) to low oxygen, combinatorial analysis of both microRNAseq and mRNAseq datasets was completed. Previous microRNAseq analysis revealed 17 microRNAs up-regulated in bMSCs (e.g. miR210, miR-221, miR-155, miR-26b, miR-31 and miR-134), whereas 18 microRNAs were up regulated in aMSCs (e.g. miR-210, miR-195, miR-181a, miR-151a, miR423 and miR-493). MiR-210 and miR-195 were the common microRNAs regulated in both MSCs. In contrast, 2 common microRNAs were found down regulated in both MSCs: miR-378c and miR-16-1-3p.

To describe the genes important for healthy cartilage maintenance that can be regulated by low oxygen in both MSCs, a comparison of healthy cartilage, and MSCs cultured in hypoxia was performed. First, all up-regulated genes in hypoxia (FC hypoxia/normoxia $>1.4$ ) in both MSCs were compared to healthy cartilage 
(Fold change healthy cartilage/disease cartilage $>1.4$ ). No genes were found in common between both MSC cell sources and healthy cartilage. However, some cell type specific genes were found in common between cartilage and aMSCs. Common genes between cartilage and aMSCs included HLA-C, KIAA1191, SLC6A9, AOX1, RHOU, HOXD4, KCNA6, TMEM92, GJA3, FGFR2, MKX, SCNN1A and VWDE. Functional analysis on these genes showed FGFR2 is involved in the FGF and WNT signaling pathways, HOXD4 is linked to transcription regulation, stem cell differentiation and embryonic skeletal formation, whereas KIAA1191 participates in the regulation of neuronal survival, differentiation and axonal outgrowth. In addition, genes in common between cartilage and bMSCs included TNFRSF11B, ERRFI1, SNX25, TIPARP, DDR1, ZNF773, ANGPT2, C7, SLC25A21 and LOC100507086. GO analyses revealed involvement in TNF signaling pathway, apoptotic signaling pathway (TNFRSF11B), SMAD and TGF $\beta$ signaling pathway (SNX5).

Common up-regulated genes between aMSCs and bMSCs included TNFRSF10D, STC2, COX7A1, NOL3-ARC, HSPB2-C11orf52 and APOA1. Functional analysis revealed involvement in the TNF mediated signaling pathway (TNFRSF10D, APOA1 and NOL3) and TGF $\beta$ signaling pathway (APOA1). Moreover, 2 of these genes are also targets of miR-210: STC2 and TNFRSF10D. Comparison of downregulated genes was also completed. No genes were regulated by both MSCs and healthy cartilage whereas 50 genes were found in common between cartilage and aMSCs (e.g. BHLHE40, TUBB3, NQO1, ITPRIP, IRF2BP2, RGS2, FRMD4A, MCM2, LPCAT4, RIN1, ETFB, UBA7, FN3KRP, LHFPL2, ACOT7, SARS2, GAMT, NCAPH, C6orf1, NTHL1, CHCHD10, LETMD1, NR4A3, IFIT3, PRSS3, FAM102B, MLKL, FOXRED2, PGAP2, C14orf159, ETV4, PARPBP, FANCD2, GALNT12, TFAP4, CCDC15, ANKRD39, LRRN4CL, MIR2116, FN3K, KRT17, OAS2, CCDC150, RNFT2, FCRLA, SEMA4A, AKR7A3, WDR88, SULT1A1 and TTLL13) and 1 gene was common between cartilage and bMSCs (e.g. IGDCC4). Gene ontology analysis exhibited that 
these genes participate in CCKR signaling, cytoskeletal regulation, insulin/IGF pathway and MAP kinase cascade whereas IGDCC4 is involved in neural crest differentiation. Additionally, 10 genes were found down regulated in both aMSCs and bMSCs: SLFN11, PRICKLE1, SLC35F2, TRIM59, AMPH, FAM46B, SHMT1, $P D G F D, A R H G A P 11 B$ and BACH2. GO analysis revealed participation of these genes in different pathways such as PDGF, MAPK, TGF $\beta$ signaling pathway (PDGFD) and WNT signaling pathway (PRICKLE1). Transcriptional regulatory activity was described for BACH2. 3 of these genes were also miR-210 gene targets: SHMT1, SLC35F2 and BACH2. Although low oxygen tension does not seem to regulate the same genes in both MSCs, it appears to coordinate specific regulatory mechanisms that are essential for chondrogenic differentiation for both aMSCs and bMSCs. In addition, a common regulatory mechanism in both MSCs controls the oxidative stress response under hypoxia.

Analysis of the top genes regulated in hypoxia was completed to obtain the hypoxic signature for each cell type. First, soft filtering was applied and genes with more than 1.4-fold change, RPKM value above 0.01 and $p$-value below 0.05 were chosen. Then, two different sets of genes were analyzed: the top 20 most expressed genes in hypoxia (according to RPKM values) and the top 20 genes with highest fold change Hypoxia/Normoxia genes. Figure 4.9.A shows the top 20 genes with the highest RPKM values in hypoxic aMSCs. Several of them have been linked to oxygen response (e.g. LDHA, LOXL2, LOX, PLOD2, PKM, THBS1, ENO1 and P4HA1), whereas some are related to bone extracellular matrix and osteoarthritis development (e.g. COL1A1, COL1A2, COL5A1, GREM1 and TGFB). Other genes participate in various cell processes such as cell metabolism, apoptosis and cell cycle (e.g MIF, ITGA5, ALDOA, CCDC80, GAPDH, PGK1 and TPI1). Moreover, several genes positioned in the top 20 genes of highest fold change between hypoxia and normoxia (Figure 4.9.B) have been associated with different cell processes (e.g. GPR146, PPFIA4, FGF11, SPAG4, VLDLR, PFKFB4, 
ALDOC, CCDC80, BNIP3, BNIP3L, ICAM5, RAB20, FBX016, FER1L4, RPL17P50 and ZNF395) whereas only a small part was linked to oxygen response (e.g NDUFA4L2, CA9, HIF3A, STC2 and ENO2). From these genes, only HIF3A is also a miR-210 target.
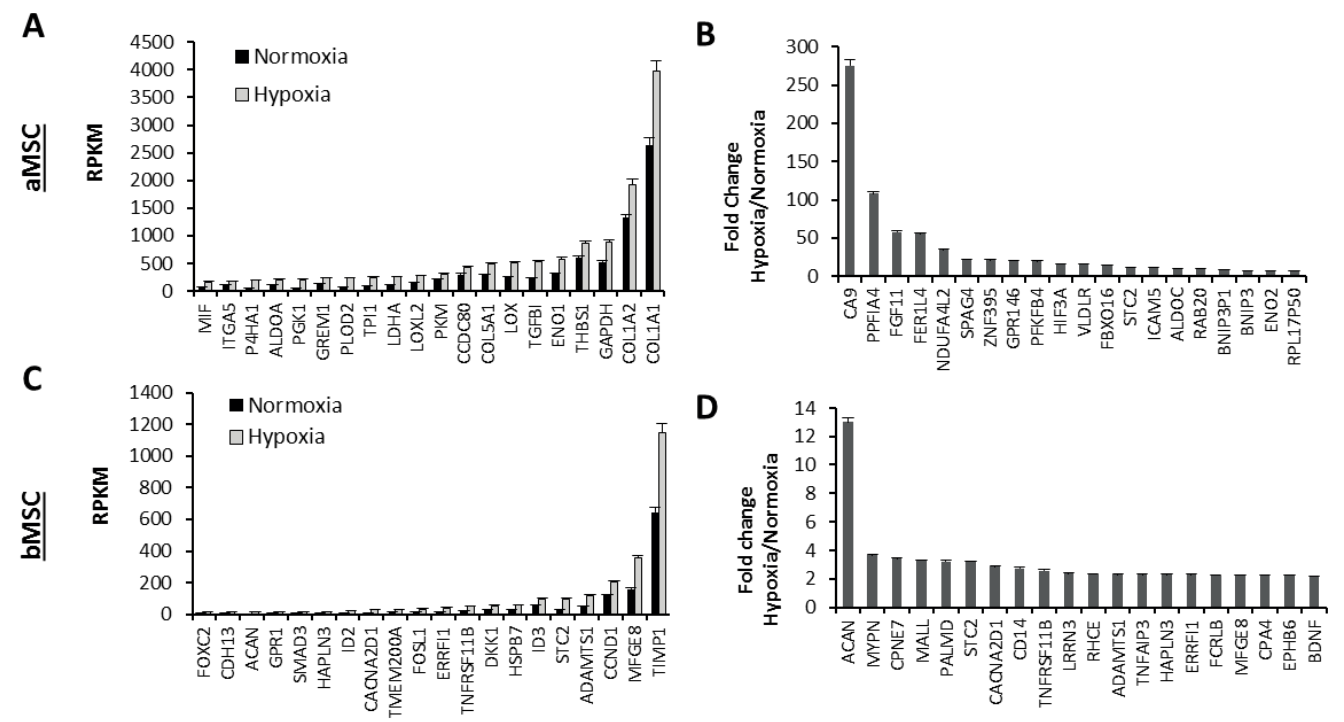

Figure 4.9. (A and C) Top 20 genes with highest RPKM values and (B and D) top20 of highest fold change between hypoxia and normoxia in hypoxic aMSCs and bMSCs.

The same analysis was completed for bMSCs (Figure 4.9.C). In this case, the 20 genes with highest RPKM in hypoxia included several genes linked to oxygen response (e.g. TIMP1, HSPB7, MFGE8, ERRFI1, STC2 and CDH13) and cartilage development/maintenance (e.g. ADAMTS1, TIMP1, DDK1, ID3, ACAN, TNFRSF11B, SMAD3 and HAPLN3), whereas the rest were linked to cell cycle (e.g. CCND1 and ID3) and other cell processes such as cell differentiation, cell proliferation, apoptosis, calcium regulation and cell transformation (e.g. FOXX2, GPR1, ID2, CACNA2D1, TMEM200A and FOSL1). Some of these genes were also found in the top 20 of highest fold change between hypoxia and normoxia (e.g. ACAN, ADAMTS1, CACNA2D1, ERRFI1, HAPLN3, MFGE8, STC2 and TNFRSF11B); genes 
that mostly participate in oxygen response and cartilage development (Figure 4.9.D). The rest of the genes participate in stress response, immune response, cancer, membrane trafficking, cell adhesion and migration, cell cycle and cytoskeletal binding (e.g. BDNF, EPHB6, CPA4, FCRLB, CPNE7, MYPN, MALL, PALMD, CD14, LRRN3, RHCE and TNFAIP3).

Interestingly, genes up regulated in each MSC depict significant differences between aMSCs and bMSCs during hypoxic response. Both MSCs up-regulate genes that participate in similar biological processes, such as hypoxia response, cell metabolism, apoptosis and cell cycle. In adittion, genes increased in bMSCs are involved in cartilage development and maintenance whereas genes increased in aMSCs are involved in bone extracellular matrix synthesis and osteoarthritis development.

\subsection{DISCUSSION}

The importance of signalling molecules such as miRNAs for cartilage development, maintenance, and destruction [71-74] has been extensively studied. Some have been found induced under hypoxic conditions including miR155, miR-424, miR-17-92, miR-21, miR-23, miR-24, miR-26, miR-103 and miR107; with miR-210 standing out in several studies as the only highly inducible miRNA under low oxygen $[75,76]$. A number of pathophysiological findings propose that a correlation does exist between hypoxia and chondrogenesis [8]. Additionally, several studies sustain that a low oxygen tension environment may be beneficial to preserve the chondrocyte phenotype of the cells during in vitro cultures, avoiding the potential de-differentiation of the cells during expansion $[8,77,78]$. However, the molecular mechanisms by which chondrocytes are regulated by oxygen tension remains poorly understood.

In this study, we have performed an integrated analysis of miRNA-seq and mRNA-seq on hypoxic mesenchymal stem cells and cartilage tissue to identify 
hypoxia-regulated miRNAs and their gene targets. First, we examined the hypoxic microRNA footprint on both aMSCs and bMSCs and concluded which miRNAs were significantly regulated in both MSCs. Then, prediction analysis was utilized to list potential and bona fide miR-210 targets in aMSCs and bMSCs. Gene ontology analysis examined the functions and regulatory mechanisms of the predicted genes. We have identified important regulatory mechanisms that control the stress response and skeletal development of the cells during low oxygen cultures. HIF1 gene targets were also analyzed against our mRNAseq data to examine which genes could be regulated by both HIF1 and miR-210 under low oxygen tension. This analysis was performed on bMSCs and also aMSCs. Subsequently, investigation of common target genes and regulatory mechanisms was performed on both MSCs. Lastly, analysis of a healthy cartilage mRNA signature was compared against genes regulated under low oxygen tension in both aMSCs and bMSCs to obtain a list of genes that could potentially enhance chondrogenic differentiation on each cell type. Some regulatory mechanisms were cell type specific whereas others were shared between both MSCs.

Thus, we have reported on various findings: i) miR-210 is the unique hypoxia induced miRNA in both bMSCs and aMSCs, ii) modestly induced miRNAs in hypoxia vary with cell type and do not participate in a specific molecular program, iii) hypoxia regulated miR-210 gene targets are involved in stress response and in cartilage development, iv) common gene targets of HIF1 and microRNA-210 play an essential roles in oxidative stress response, cell cycle regulation and skeletal development and it appears that HIF1 and miR-210 act in concert in the regulation of many of these genes, v) miR-210 may play an important role in the inflammatory response and cartilage homeostasis in healthy cartilage tissues, and vi) aMSCs and bMSCS have a different microRNA and mRNA footprint under low oxygen. 
miR-210 was the dominant miRNA involved in regulation of gene expression responses in both aMSCs and bMSCs, at least part of its effects on gene expression are regulated in concert with the transcription factor HIF1. Although, low amounts of genes were shown to overlap in both MSCs, an important overlap in cellular processes and functions was found. This was achieved by the interaction with other genes that may have redundant functions but are expressed in a cell type specific manner. Hypoxia regulates two important cellular functions, skeletal development and oxidative stress response in MSCs. The first set of genes is involved in preventing hypertrophic and ossification by increasing cartilage ECM protein synthesis (e.g. COL2A1 and ACAN) [37, 79] through regulatory pathways, such as WNT and TGF $\beta[39,80]$. The remaining genes are involved in the cell response to oxidative stress [41] mediated by increasing anti-inflammatory response [81] and cell cycle regulation [82] to prevent apoptosis and DNA damage. Increased expression of genes involved in WNT, FGF and TGF $\beta$ signaling pathways [66] might be relevant for chondrogenic differentiation. These signaling pathways have all been implemented in maintenance of healthy cartilage and/or regulation of chondrogenic differentiation (Chapter 2). Thus, the beneficial effects of hypoxia on chondrogenic differentiation can be at least in part attributed to the regulation of these signaling pathways.

Although all these genes have been described in the literature, none were previously linked to miR-210. The present work suggested a link of WNT11, WISP1 and BACH2, found by target prediction analysis, and SHMT1 and SLCC3F2, found by mRNAseq, as bona fide miR-210 targets essential for cartilage development and oxidative stress response regulation. This regulatory mechanism may promote cartilage development through releasing a brake on ECM protein synthesis, proteoglycan deposition, aggrecan and collagen type II synthesis. Although $\mathrm{BACH} 2$ has not been described as a participant in any 
pathway involved in cartilage development, its inhibition by miR-210 during hypoxia may help in inducing an anti-inflammatory response by inhibiting oxidative stress induced apoptosis and by decreasing HMOX1 gene expression. Moreover, the difference in gene expression and pathways regulated during low oxygen response suggested a difference in the chondrogenic potential of aMSCs and bMSCs.

Though the identity of genes shows little overlap between MSCs, the biological processes and functions regulated by both cells in low oxygen are very similar. Suggesting the participation of several genes, not detected in this study, in similar functions as those previously described. Furthermore, common genes between MSCs and cartilage diverged in both oxygen conditions. Genes increased in hypoxia are mostly involved in cartilage regulatory mechanisms, while genes decreased in hypoxia participate in several biological processes not related to cartilage (e.g. glucose and intestinal mucosa regulation, and neural crest differentiation).

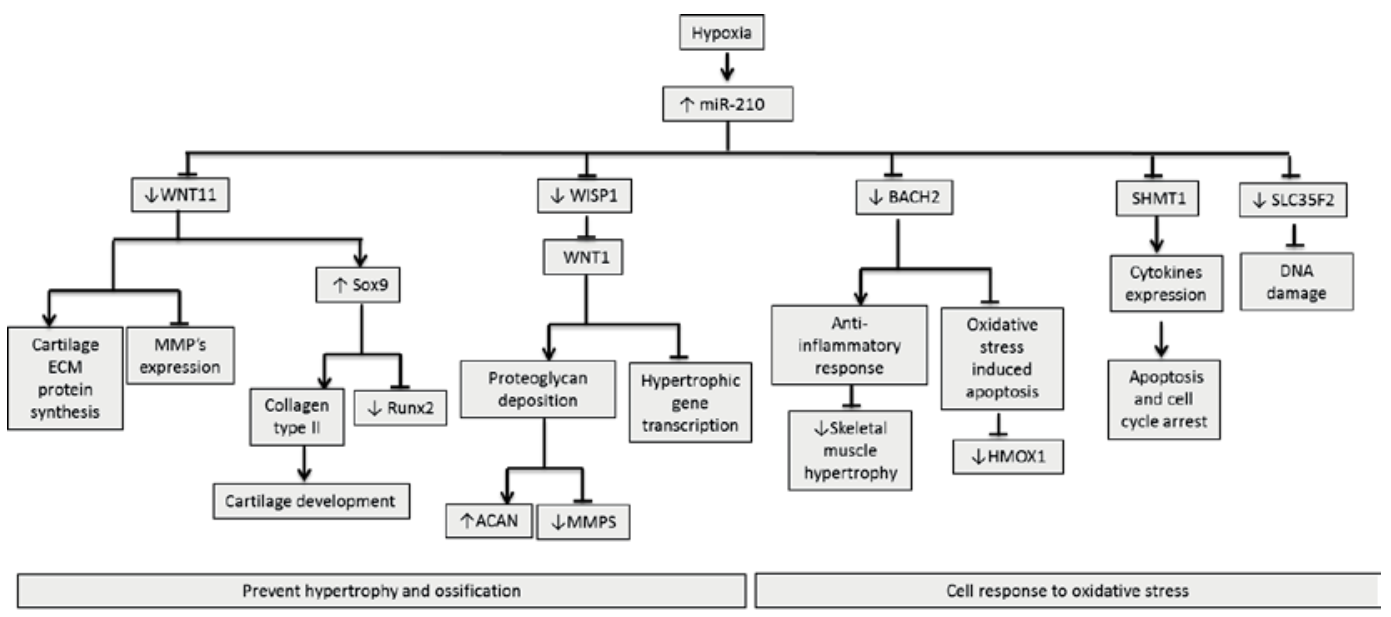

Figure 4.10 Identification of genes that can be modulated by miR-210 expression. To summarize our findings a model was created using the information obtained along the study. 
Nevertheless, genes that are commonly regulated in both MSCs indicated that during the hypoxic response they are involved in preventing premature endochondral ossification of the cells whereas up-regulated genes are involved in cartilage maintenance of healthy cartilage. Our results suggest that some matrix degradation genes are direct targets of miR-210, suggesting that miR-210 can be potentially used to target these genes avoiding progression of disease. Interestingly, high induction of miR-210 could cause an inflammatory response on the cells and negative regulation of cartilage development.

One of the potential limitations of our study is the small sample number $(n=3)$ used. Although RNAseq is expensive; a larger sample number would increase reliability of the results. Moreover, only one culture condition (monolayer) was examined in this study. Further analysis on different cell culture conditions (e.g. chondrogenic differentiation, 3D scaffolds, etc) and oxygen levels will help to validate the results observed in this study.

The results from this combinatorial analysis of microRNAseq and mRNAseq may be a useful tool to control identified potential gene targets to enhance chondrogenic differentiation of MSCs in different cultures or help in promoting in vitro cartilage regeneration. Several of the genes found in this analysis can be used for pharmacological targeting or genetic approaches in order to promote a stable and better chondrogenic phenotype of mesenchymal stem cells. 


\section{REFERENCES}

1. Giaccia A, Simon M, Johnson R: The biology of hypoxia: the role of oxygen sensing in development, normal function, and disease. Genes Dev 2004, 18:2183-2194.

2. Markway B, Cho H, Anderson D, Holden P, Ravi V, Little C, Johnstone B: Reoxygenation enhances tumour necrosis factor alpha-induced degradation of the extracellular matrix produced by chondrogenic cells. Eur Cell Mater 2016, 31:425-439.

3. Kean T, Mera H, Whitney G, MacKay D, Awadallah A, Fernandes R, Dennis J: Disparate response of articular- and auricular-derived chondrocytes to oxygen tension. Connect Tissue Res 2016, 57:319-333.

4. Masoud G, Li W: HIF-1 $\alpha$ pathway: role, regulation and intervention for cancer therapy. Acta Pharma Sin B 2015, 5:378-389.

5. Mircea I, Huang X: miR-210: Fine-Tuning the Hypoxic Response. Adv Exp Med biol 2015, 772:205-227.

6. Falah M, Nierenberg G, Soudry M, Hayden M and Volpin G: Treatment of articular cartilage lesions of the knee. Int Orthopedic 2010:34:621-630.

7. Camilleri ET, Dudakovic A, Riester SM, Galeano-Garces C, Paradise CR, Bradley EW, McGee-Lawrence ME, Im HJ, Karperien M, Krych AJ, Westendorf JJ, Larson AN, van Wijnen AJ.: Loss of histone methyltransferase Ezh2 stimulates an osteogenic transcriptional program in chondrocytes but does not affect cartilage development. J Biol Chem 2018:19001-19011.

8. Galeano-Garces C, Camilleri E, Riester SM, Dudakovic A, Larson DR, Qu W, Smith J, Dietz AB, Im HJ, Krych AJ, Larson AN, Karperien M, van Wijnen AJ.: Molecular Validation of Chondrogenic Differentiation and Hypoxia Responsiveness of PlateletLysate Expanded Adipose Tissue-Derived Human Mesenchymal Stromal Cells. Cartilage 2017:283-299.

9. Georgi N, Taipaleenmaki H, Raiss CC, Groen N, Portalska KJ, van Blitterswijk C, de Boer J, Post JN, van Wijnen AJ, Karperien M.: MicroRNA Levels as Prognostic Markers for the Differentiation Potential of Human Mesenchymal Stromal Cell Donors. Stem Cells Dev 2015:1946-1955.

10. Donker RB, Mouillet JF, Nelson DM, Sadovsky Y.: The expression of Argonaute2 and related microRNA biogenesis proteins in normal and hypoxic trophoblasts. Mol Hum Reprod 2007:273-279.

11. Fasanaro P, D’Alessandra Y, Di Stefano V, Melchionna R, Romani S, Pompilio G, Capogrossi MC, Martelli F.: MicroRNA-210 modulates endothelial cell response to hypoxia and inhibits the receptor tyrosine kinase ligand Ephrin-A3. J Biol Chem 2008:15878-15883.

12. Ivan M, Harris AL, Martelli F, Kulshreshthaa R: Hypoxia response and microRNAS: no longer two separate worlds. J Cell Mol Med 2008:1426-1431.

13. Ivan M, Huang X: miR-210: Fine Tuning The hypoxic Response. Adv Exp Med Biol 2014:205-227.

14. Dudakovic A, Camilleri E, Riester SM, Lewallen EA, Kvasha S, Chen X, Radel DJ, Anderson JM, Nair AA, Evans JM, Krych AJ, Deyle DR, Stein JL, Im HJ, Cool SM, Westendorf JJ, Kakar S, Dietz AB, van Wijnen A: High-resolution molecular validation of self-renewal and spontaneous differentiation in clinical-grade adipose-tissue derived human mesenchymal stem cells. J Cell Biochem 2014, 115:1816-1828.

15. Lewallen EA, Bonin C, Li X, Smith J, Karperien M, Larson AN, Lewallen DG, Cool SM, Westendorf JJ, Krych AJ, Leontovich AA, Im HJ, van Wijnen AJ: The synovial 
microenvironment of osteoarthritic joints alters RNA-seq expression profiles of human primary articular chondrocytes. Gene 2016:456-464.

16. Riester SM, Dudakovic A, Camilleri ET, Wang W, Xu F, Thaler R, Evans JM, Zwartbol R, Briaire-de Bruijn IH, Maran A, Folpe AL, Inwards CY, Rose PS, Shives TC, Yaszemski MJ, Sim FH, Deyle DR, Larson AN, Galindo MA, van Wijnen AJ: Hypoxia-related microRNA-210 is a diagnostic marker for discriminating osteoblastoma and osteosarcoma. J Orthop Res 2017:1137-1146.

17. Martin M: Cutadapt removes adapter sequences from high-throughput sequencing reads. Journal Bioinformatics in Action. 2011.

18. Langmead B, Trapnell C, Pop M, Salzberg SL: Ultrafast and memory-efficient alignment of short DNA sequences to the human genome. Genome Biol 2009:R25.

19. Friedländer MR, Chen W, Adamidi C, Maaskola J, Einspanier R, Knespel S, Rajewsky N: Discovering microRNAs from deep sequencing data using miRDeep. Nat Biotechnol 2008:407-415.

20. Kim D, Pertea G, Trapnell C, Pimentel H, Kelley R, Salzberg SL.: TopHat2: accurate alignment of transcriptomes in the presence of insertions, deletions and gene fusions. Genome biology 2013:14:R36.

21. Anders S, Pyl PT, Huber W: HTSeq-a Python framework to work with highthroughput sequencing data. Bioinformatics 2015:166-169.

22. Oliveros J: Venny. An interactive tool for comparing lists with Venn's diagrams.; 20072015.

23. Starruss J, de Back W, Brusch L, Deutsch A: Morpheus: a user-friendly modeling environment for multiscale and multicellular systems biology. Bioinformatics 2014, 30:1331-1332.

24. Ashburner M, Blake JA, Botstein D, Butler H, Cherry JM, Davis AP, Dolinski K, Dwight SS, Eppig JT, Harris MA, Hill DP, Issel-Tarver L, Kasarskis A, Lewis S, Matese JC, Richardson JE, Ringwald M, Rubin GM, Sherlock G.: Gene ontology: tool for the unification of biology. The Gene Ontology Consortium. Nature Genetics 2000:25-29.

25. Consortium TGO: Expansion of the Gene Ontology knowledgebase and resources. Nucleic Acids Res 2017:D331-D338.

26. Szklarczyk D, Kuhn M, Simonovic M, Roth A, Minguez P, Doerks T, Stark M, Muller J: The STRING database in 2011: functional interaction networks of proteins, globally integrated and scored. . Nucleic Acids Res 2011:39:D561-D568.

27. Warde-Farley D, Comes O, Zuberi K, Badrawi R, Chao P, Franz M, Grouios C, Kazi F, Lopes CT, Maitland A, Mostafavi S, Montojo J, Shao Q, Wright G, Bader GD, Morris Q: The GeneMANIA prediction server: biological network integration for gene prioritization and predicting gene function. Nucleic Acids Research 2010:W214-220.

28. Gao S, Chen L, Zhao Y, Sun L, Cao M, Huang Y, Niu Q, Wang F, Yuan C, Li C, Zhou X.: Brain-derived neurotrophic factor: A steroidogenic regulator of Leydig cells. J Cell Physiol 2019.

29. Hultgårdh-Nilsson A, Chakravarti S.: The small leucine-rich repeat proteoglycans in tissue repair and atherosclerosis. J Intern Med 2015:447-461.

30. Medina R, Liu CG, van Wijnen AJ, Croce CM, Stein GS: microRNA 221 and 222 bypass quiescence and compromise cell survival Carcer Res 2008:2773-2780.

31. Ponsuksili S, Murani E, Trakooljul N, Schwerin M, Wimmers K: discovery of candidate genes for muscle traits based on GWAS supported by eQTL-analysis. Int J Biol Sci 2014:327-337. 
32. Kirikoshi H, Katoh M.: Molecular cloning and characterization of human WNT11. Int J Mol Med 2001:651-656.

33. Toyama T, Koga H, Wands JR, Kim M.: Noncanonical Wnt11 inhibits hepatocellular carcinoma cell proliferation and migration. Mol Cancer Res 2010:254-265.

34. Ryu JH, Chun JS: Opposing roles of WNT-5A and WNT-11 in interleukin-1beta regulation of type II collagen expression in articular chondrocytes. J Biol Chem 2006:22039-22047.

35. Choi D, Shin JH, Ryu CM, Yu HY, Kim A, Lee S, Lim J, Shin DM, Choo MS.: Downregulation of WNT11 is associated with bladder tissue fibrosis in patients with interstitial cystitis/bladder pain syndrome without Hunner lesion. Sci Rep 2018:9782.

36. Chen YZ, Zheng Y, Zheng GK, Chen RQ, Lin M, Huang LF, Huang C, Song D, Wu BQ.: WISP1 silencing confers protection against epithelial-mesenchymal transition of renal tubular epithelial cells in rats via inactivation of the wnt $/ \beta$-catenin signaling pathway in uremia. J Cell Physiol 2018.

37. Blom AB, van Lent PL, van Beuningen HM, Geurts J, Takahashi N, van der Kraan PM, van de Loo FA, Schreurs BW, Clements K, Newham P, van den Berg WB.: Involvement of the Wnt signaling pathway in experimental and human osteoarthritis: prominent role of Wnt-induced signaling protein 1. Arthritis Rheum 2009:501-512.

38. Liu ZJ, Tan Y, Xiao M, Zhang J, Radtke F, Velazquez OC.: Inhibition of fibroblast growth by Notch1 signaling is mediated by induction of Wnt11-dependent WISP-1. Send to 2012:e38811.

39. Oyake T, Motohashi H, Hayashi N, Hoshino H, Nishizawa M, Yamamoto M, Igarashi K: Bach proteins belong to a novel family of BTB-basic leucine zipper transcription factors that interact with MafK and regulate transcription through the NF-E2 site. Mol Cell Biol 1996:6083-6095.

40. Zhang H, Miranda RN, Medeiros LJ, McCarty N.: Bifurcated BACH2 control coordinates mantle cell lymphoma survival and dispersal during hypoxia. Blood 2017:763-776.

41. Shukla M, White SA, Singh PP, Reid AM, Catania S, Pidoux AL, Allshire RC.: Centromere DNA Destabilizes H3 Nucleosomes to Promote CENP-A Deposition during the Cell Cycle. Curr Biol 2018:3924-3936.

42. Yu KY, Norman DA, Vig E, Goebl MG, Harrington MA.: Cutting edge: mouse pellino-2 modulates IL-1 and lipopolysaccharide signaling. 2002:4075-4078.

43. He Z, Song A, Cui X, Ma Z, Zhang Z.: Identification of LINC01234 and MIR210HG as novel prognostic signature for colorectal adenocarcinoma. J Cell Physiol 2018:27424.

44. Ishida Y, Kabashima K.: Cutaneous angiosarcoma: update on biology and latest treatment. Curr Opin Oncol 2018:107-112.

45. Yu P, Huang L, Yang L, Du Y.: MiR210 as a potential molecular target to block invasion and metastasis of gastric cancer. Med Hypotheses 2015:209-212.

46. Li C, Wang Y, Jing S, Yang C, Sun G, Liu Q, Cheng Y, Wang L: miR-210 regulates esophageal cancer cell proliferation by inducing $\mathrm{G} 2 / \mathrm{M}$ phase cell cycle arrest through targeting PLK1. Mol Med Rep 2014 2099-2104.

47. Kuokkanen S, Ojalvo L, Benard L, Santoro N, Pollard JW.: Genomic profiling of microRNAs and messenger RNAs reveals hormonal regulation in microRNA expression in human endometrium. Biol Reprod 2010:791-801. 
48. Chen J, Wang D, Xu Y, Gao H, Tan W, Wang C: Contribution of regulatory T cells to immune tolerance and association of microRNA-210 and Foxp3 in preeclampsia. Mol Med Rep 2018:9733.

49. Silakit R, Thongchot S, Loilome W, Techasen A, Ungarreevittaya P, Khuntikeo N, Yongvanit P, Yang JH, Kim NH, Yook JI, Namwat N.: Potential role of HIF-1-responsive microRNA210/HIF3 axis on gemcitabine resistance in cholangiocarcinoma cells. PLoS One 2018.

50. Qu Y, Huang W: Effects of microRNA-210 on the diagnosis and treatment of prostate cancer. Mol Med Rep 2018.

51. Li BY, Zhao WS, Zhang L, Zhou HJ, Zou YC, Zhang T.: MicroRNA-210 negatively regulates the radiosensitivity of nasopharyngeal carcinoma cells. Mol Med Rep 2017.

52. Liu C, Tang X: Downregulation of microRNA-210 inhibits osteosarcoma growth in vitro and in vivo. Mol Med Rep2015.

53. Zhang Z, Dai H, Walsh RM, Imakura M, Schelter J, Burchard J, Dai X, Chang AN, Diaz RL, Marszalek JR, Bartz SR, Carleton M, Cleary MA, Linsley PS, Grandori C.: MicroRNA miR210 modulates cellular response to hypoxia through the MYC antagonist MNT. Cell Cycle 2009:2756-2768.

54. Kouvaras E, Siasios I, Malizos K, Koukoulis GK, Ioannou M: Hypoxia-inducible factor 1alpha and vascular endothelial growth factor in cartilage tumors. Biotech Histochem 2019:1-7.

55. Heo JH, Kim IR, Park BS, Kim YD.: Combined Treatment with Low-Level Laser and rhBMP-2 Promotes Differentiation and Mineralization of Osteoblastic Cells under Hypoxic Stress. Tissue Eng Regen Med 2018:793-801.

56. Liu W, Zhao XY, Chen GQ: Targeted genes and interacting proteins of hypoxia inducible factor-1. Int J Biochem Mol Biol 2012:165-178.

57. Murahashi Y, Kobayashi H, Makii Y, Iba K, Yamashita T, Tanaka S, Saito T.: Intraarticular administration of I $\mathrm{KB} \alpha$ kinase inhibitor suppresses mouse knee osteoarthritis via downregulation of the NF- $\kappa B / H I F-2 \alpha$ axis. Sci Rep 2018.

58. Fisch KM, Alvarez-Garcia 0, Akagi R, Saito M, Muramatsu Y, Sasho T, Koziol JA, Su AI, Lotz MK: Identification of transcription factors responsible for dysregulated networks in human osteoarthritis cartilage by global gene expression analysis. Osteoarthritis Cartilage 2018:1531-1538.

59. Lacedonia D, Pia Palladino G, Gallo C, Carpagnano GE, Sabato R, Foschino Barbaro MP.: MicroRNA expression profile during different conditions of hypoxia. Oncotarget 2018:35114-35122.

60. Wang Y, Liu Y, Zhao W, Zhang Z, Lu M, Zhang W.: Decrease of miR-195 Promotes Chondrocytes Proliferation and Maintenance of Chondrogenic Phenotype via Targeting FGF-18 Pathway. Int J Mol Sci 2017:E975.

61. Gu YL, Wen LT, Zhu GX, Qian MQ: miR-195 inhibits the proliferation and migration of chondrocytes by targeting GIT1. Mol Med Rep 2017:194-200.

62. Bai R, Zhao ZQ, Liu WL, Jian DM.: MicroRNA-195 induced apoptosis in hypoxic chondrocytes by targeting hypoxia-inducible factor 1 alpha. Eur Rev Med Pharmacol Sci 2015:545-551.

63. Ishida MS, Yagi S, Nishimoto S, Kozuka C, Fukuda D, Soeki T, Masuzaki T, Tsutsui M, Sata M: MicroRNA-378 Regulates Adiponectin Expression in Adipose Tissue: A New Plausible Mechanism. PLoS ONE 9 2014:e111537. . 
64. Feng QQ, Zhou Y, Zhang H, Long C: miR-16-1-3p targets TWIST1 to inhibit cell proliferation and invasion in NSCLC. Bratisl Lek Listy 2018:60-65.

65. Li R, He CQ, Yang YQ, Guo H, Chen Y, Du TH.: Upregulation of fibroblast growth factor 1 in the synovial membranes of patients with late stage osteoarthritis. Genet Mol Res 2015:11191-11199.

66. de Seny D, Charlier E, Neuville S, Lutteri L, Le Goff C, Malaise D, Malaise O, Chapelle JP, Relic B, Malaise MG.: Apolipoprotein-A1 as a damage-associated molecular patterns protein in osteoarthritis: ex vivo and in vitro pro-inflammatory properties. PLoS One 2015:e0122904.

67. Gelse K, Cipa F, Swoboda B, Carl HD, Olk A, Hennig FF, Klinger P: Molecular differentiation between osteophytic and articular cartilage--clues for a transient and permanent chondrocyte phenotype. Osteoarthritis Cartilage 2012:162-171.

68. Ao JE, Zhou Y, Zhao R, Yang CM.: Hypoxia-inducible factor 1 regulated ARC expression mediated hypoxia induced inactivation of the intrinsic death pathway in p53 deficient human colon cancer cells. Biochem Biophys Res Commun 2012:913-917.

69. Micheau 0: Regulation of TNF-Related Apoptosis-Inducing Ligand Signaling by Glycosylation. Int J Mol Sci 2018:E715.

70. Sumiyoshi K, Kubota S, Ohgawara T, Kawata K, Nishida T, Shimo T, Yamashiro T, Takigawa M.: Identification of miR-1 as a micro RNA that supports late-stage differentiation of growth cartilage cells. Biochem Biophys Res Commun 2010:286290.

71. Ukai T, Akutsu H, Umezawa A, Mochida J: MicroRNA-199a-3p, microRNA-193b, and microRNA-320c are correlated to aging and regulate human cartilage metabolism. J Orthop Res 2012:1915-1922.

72. Song J, Kim D, Han J, Chun CH, Jin EJ.: MicroRNA-181b regulates articular chondrocytes differentiation and cartilage integrity . Biochem Biophys Res Commun 2013:210-214.

73. Vonk LA, Dhert WJ, Saris DB, Creemers LB: Overexpression of hsa-miR-148a promotes cartilage production and inhibits cartilage degradation by osteoarthritic chondrocytes. Osteoarthritis Cartilage 2014:145-153.

74. Kulshreshtha R, Ferracin M, Wojcik SE, Garzon R, Alder H, Agosto-Perez FJ, Davuluri R, Liu CG, Croce CM, Negrini M, Calin GA, Ivan M.: MicroRNA signature of hypoxia. Mol Cell Biol 2007:1859-1867.

75. Camps C, Buffa FM, Colella S, Moore J, Sotiriu C, Sheldon H, Harris AL, Gleadle JM, Ragoussis J: hsa-miR-210 is induced by hypoxia and is an independent prognostic factor in breast cancer. Clin Cancer Res 2008:1340-1348.

76. Leijten J, Georgi N, Moreira Texeira L, van Blitterswijk C, Post J, Karperien M: Metabolic programming of mesenchymal stromal cells by oxygen tension directs chondrogenic cell fate. PNAS 2014, 38:13954-13959.

77. Leijten J, Moreira Texeira L, Landman E, van Blitterswijk C, Karperien M: Hypoxia Inhibits Hypertrophic Differentiation and Endochondral Ossification in Explanted Tibiae. PLOS One 2012.

78. Liu S, Zhang E, Yang M, Lu L: Overexpression of Wnt11 promotes chondrogenic differentiation of bone marrow-derived mesenchymal stem cells in synergism with TGF-beta. Mol Cell Biochem 2014, 390:123-131. 
79. Paradise CR, Galeano-Garces C, Galeano-Garces D, Dudakovic A, Milbrandt TA, Saris DBF, Krych AJ, Karperien M, Ferguson GB, Evseenko D, et al: Molecular characterization of physis tissue by RNA sequencing. Gene 2018, 668:87-96.

80. Gupta R, Yang Q, Dogra SK, Wajapeyee N: Serine hydroxymethyl transferase 1 stimulates pro-oncogenic cytokine expression through sialic acid to promote ovarian cancer tumor growth and progression. Oncogene 2017, 36:4014-4024.

81. Wu S, Zhang G, Li P, Chen S, Zhang F, Li J, Jiang C, Chen X, Wang Y, Du Y, et al: miR-198 targets SHMT1 to inhibit cell proliferation and enhance cell apoptosis in lung adenocarcinoma. Tumour Biol 2016, 37:5193-5202. 


\section{CHAPTER 5}

\section{5. miRNA-210 promotes chondrogenesis of MSCs}




\section{ABSTRACT}

Articular Cartilage has limited capacity to respond to damage limiting the overall capacity to heal and repair. Although current techniques have shown interesting results, they still present major weaknesses. Thus, an optimal tissue engineering strategy that regenerates the natural cartilage structure is required for a successful cartilage tissue repair. Hypoxia has been described as a key factor to preserve cartilage integrity whilst a large number of microRNAs have been implicated in regulating chondrogenesis. Previously, we identified miR-210 as the unique hypoxia induced miRNA in adipose and bone marrow derived Mesenchymal Stem Cells (MSCs), which gene targets are mostly involved in the regulation of cartilage homeostasis and oxidative stress responses. Its expression is strongly upregulated during chondrogenic differentiation of MSCs. Based on these observations; we hypothesize that preconditioning of MSCs with miR-210 before chondrogenic exposure may accelerate early stages of chondrogenesis and avoid hypertrophic differentiation of the cells. Interestingly, under low oxygen cultures overexpression of miR-210 enhanced chondrogenesis of MSCs as good as TGF $\beta 1$. Chondrogenesis improvement was evidenced by increased expression of cartilaginous markers, proteoglycan deposition and collagen II protein content. In both oxygen conditions, the combined stimulation of MSCs with TGF $\beta 1$ and miR-210 decreased the synthesis and deposition of important chondrogenic matrix proteins such as collagen II and aggrecan while increasing the expression of the fibroblastic marker collagen I. Functional experiments revealed miR-210 importance for early chondrogenesis of MSCs during in vitro cultures. Hence, exogenous miR-210 expression can be used instead of chondrogenic differentiation cocktail in standard or low oxygen cultures to promote chondrogenesis of MSCs while inhibiting their hypertrophic differentiation. This potential treatment can help in developing improved protocols for the formation of hyaline cartilage using MSCs. 


\subsection{INTRODUCTION}

Articular Cartilage is composed of a dense extracellular matrix (ECM) made of $80 \%$ water, $18 \%$ type II collagen and proteoglycans, and a small portion of other proteins. Chondrocytes, specialized cells which role is to develop, maintain and repair the ECM, encompass only $2-3 \%$ of the articular cartilage volume [1]. However, chondrocytes have limited potential for replication and limited capacity to respond to damage [2], negatively impacting the overall capacity of articular cartilage to heal and repair. Although current techniques including osteochondral grafting and cellular therapies such as autologous chondrocyte implantation have shown promising results, they still present major weaknesses. Thus, an optimal tissue engineering strategy that regenerates the natural cartilage structure is required for successful cartilage tissue repair. Much attention has been paid to the use of mesenchymal Stem Cells (MSCs) as an alternative cell source for engineering new cartilage [3]. This has started the exploration of environmental factors like oxygen tension and/or additives like signaling molecules to boost chondrogenic differentiation of mesenchymal stem cells [4]. One of these environmental factors is oxygen tension. Oxygen tension has been shown to stimulate cartilage formation [1] by primary chondrocytes as well as MSCs. The exact mechanism is however still largely unknown. Several studies have demonstrated that miRNAs are involved in the hypoxic response and can contribute to the repression of specific genes under low oxygen conditions [5]. Also, hypoxia has been described as a key factor to preserve cartilage integrity whilst a large number of microRNAs have been implicated in regulating pre and post-natal chondrogenesis [6].

We have identified miR-210 as the unique hypoxia induced miRNA in both bMSCs and aMSCs (Chapter 4). Its gene targets are mostly involved in the regulation of a stress response, in preventing hypertrophy, degradation and ossification of cartilage. Nevertheless, during the hypoxic response, direct gene targets of miR- 
210 are involved in avoiding progression of disease and preventing earlier endochondral ossification of the cells. Despite several microRNAs have been described to participate during chondrogenesis of cells [7], we found hypoxic regulated miR-210 to be essential in the regulation of genes in charge of several functions necessary for in vitro cartilage development. Our goal is to precondition MSCs using miR-210 before chondrogenic exposure to accelerate early stages of chondrogenesis and avoid hypertrophic differentiation of the cells. Inhibitory experiments will also be conducted to study the effect and the relevance of miR-210 during chondrogenesis of MSCs.

\subsection{MATERIALS AND METHODS}

\subsubsection{Monolayer and high-density pellet cultures}

For cell harvest and expansion, same methods and protocols described in section 4.2.1 (Chapter 4) were used. Both MSCs were expanded in growth media. For bMSCs this media consisted of DMEM with $4.5 \mathrm{~g} / \mathrm{L}$ glucose and sodium pyruvate (Corning, Corning NY), supplemented with 10\% fetal bovine serum (Atlanta Biologicals, Atlanta, GA) and 1\% penicillin/streptomycin (Gibco/Thermo Fisher Scientific). Maintenance media for aMSCs was composed of advanced MEM (Gibco/Thermo Fisher Scientific, Waltham, MA) supplemented with 5\% human platelet lysate (PL-Max, MillCreek Life Sciences, Rochester, MN), 1\% penicillin/streptomycin, 1\% Glutamax (Gibco/Thermo Fisher Scientific), and $0.2 \%$ heparin (Baxter, Deerfield, IL). For monolayer culture, bMSCs were plated in 6-well plates at a density of 3,000 cells $/ \mathrm{cm}^{2}$. Cells were cultured using standard techniques in T- $175 \mathrm{~cm}^{2}$ flasks at $37^{\circ} \mathrm{C}, 95 \%$ humidity, and $5 \% \mathrm{CO}_{2}$ until they reached $80 \%$ confluence. Prior to each experiment, cells were detached from T175 flasks by trypsinization using TrypLE Express (Gibco/Thermo Fisher Scientific). High-density cellular aggregates were performed using pellet cultures in accordance with a protocol published previously [6]. To form the pellets, 250,000 cells were seeded in 96 round-bottom well plates followed by a 
centrifugation step to initiate pellet formation. Pellets were maintained at $37^{\circ} \mathrm{C}$, 95\% humidity, and 5\% $\mathrm{CO}_{2}$ until cells coalesced. Both cultures were kept under standard culture conditions. Media was replaced every 3 to 4 days.

\subsubsection{Cell culture under Normoxia and Hypoxia conditions}

To evaluate expression of bMSCs in response to oxygen tension, both cells were plated in monolayer or in high-density pellet cultures and maintained for up to 21 days in maintenance media under either normoxic (20\% oxygen) or hypoxic ( $2 \%$ oxygen) conditions using an integrated system with a cell culture hood and an incubator accessible through a gas-lock (I-Glove, BioSpherix, New York, NY).

\subsubsection{Chondrogenesis of bMSCs.}

Chondrocyte differentiation was induced in high-density pellets. Following pellet formation, maintenance media was exchanged with chondrogenic differentiation media which consisted of culture media supplemented with $40 \mathrm{mg} / \mathrm{mL}$ of Lproline, $50 \mathrm{mg} / \mathrm{mL}$ Insulin Transferrin Selenium-premix, $50 \mathrm{mg} / \mathrm{mL}$ of ascorbic acid, $10 \mathrm{ng} / \mathrm{mL}$ of TGF- $\beta 1$, and $0.1 \mu \mathrm{M}$ dexamethasone. MSCs were allowed to differentiate up to 21 days. Medium was refreshed every 3 to 4 days. Total RNA was isolated using the miRNeasy Micro Kit following the instructions of the manufacturer.

\subsubsection{Functional analysis of miR-210.}

Gain and loss of function experiments were performed in bMSCs. Before transfection, all cells were seeded in monolayer culture and maintained in culture media until $80 \%$ confluency was reached. Due to the absence of endogenous miR-210 in normoxia, this condition was used for gain of function experiments, whilst low oxygen conditions under which miR-210 is induced and abundantly expressed, were used for loss of function experiments. First, a dose response experiment was completed to examine toxicity of the miRNA transfection on the MSCs. Different concentrations: 5, 10, 20 and $50 \mu \mathrm{M}$ of MISSION hsa-miR-210 mimic (HMI0373, Sigma Aldrich, Saint Louis, MN, USA), 
MISSION hsa-miR-210 inhibitor (HSTUD0373, Sigma Aldrich) and MISSION Negative control miRNA (HMC0002, Sigma Aldrich) were used. Mature sequence used in both miR-210 mimic and inhibitor was CUGUGCGUGUGACAGCGGCUGA whereas negative control miRNA mature sequence was GGUUCGUACGUACACUGUUCA. Transfections were performed using Lipofectamine RNAiMAX (Thermo Fisher, Waltham, MA, USA) following manufacturer's instructions and using a 2 to 1 Lipofectamine siRNA ratio. Optimal transfection dose was calculated from these experiments. A stimulatory dose of $20 \mu \mathrm{M}$ of miR-210 mimic was used for gain of function experiments whereas $10 \mu \mathrm{M}$ of miR-210 inhibitor was selected for loss of function experiments. In both experiments gene expression, protein analysis and histological analysis were performed.

An outline for the experimental set-up of this chapter is included in Figure S5.1.

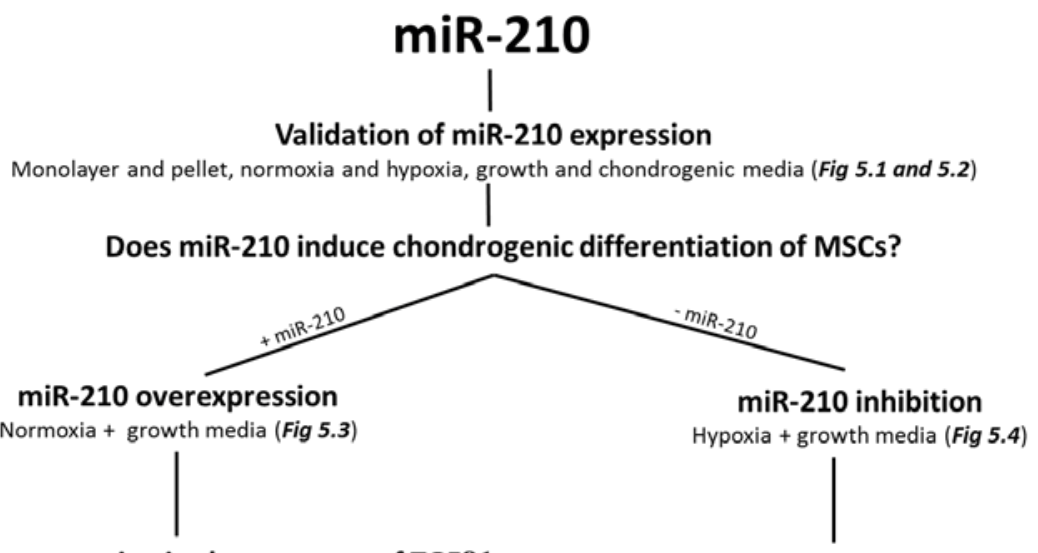

miR-210 overexpression in the presence of TGF $\beta 1$

Normoxia + chondrogenic media (Fig 5.5 and 5.6)

miR-210 inhibition in the presence of TGF $\beta 1$ Hypoxia + chondrogenic media (Fig 5.5 and 5.6)

\section{miR-210 overexpression in hypoxia}

Hypoxia \pm chondrogenic media (Fig 5.7)

Figure S.5.1. Experimental outline. 


\subsubsection{Gene expression analysis}

For pellet cultures, at least 4 pellets were pooled from each condition to obtain sufficient RNA. Pooled pellets from each time point were lysed using an 18-gauge needle and Qiazol lysis buffer (Qiagen, Hilden, Germany) to yield an RNA sample for one biological replicate. For monolayer cultures, one well from the 6-well plate was collected as one biological replicate. Three different biological replicates for each experimental condition (e.g., 3 samples for normoxia and 3 samples for hypoxia) were used. Total RNA was isolated using the miRNeasy Micro Kit (Qiagen, Hilden, Germany) following the instructions of the manufacturer. RNA concentrations and purity levels were measured using a NanoDrop (Thermo Fisher Scientific).

Table 5.1 miRNA Primers sequences used for gene expression analysis.

\begin{tabular}{|lll|}
\hline Gene ID & Forward Primer Sequence & Reverse Primer Sequence \\
\hline miR-191-5p & CAACGGAATCCCAAAAGCAGCTG & U6 Reverse Primer - TaKaRa \\
miR-210-3p & CTGTGCGTGTGACAGCGGCTGA & U6 Reverse Primer - TaKaRa \\
miR-21-5p & TAGCTTATCAGACTGATGTTGA & U6 Reverse Primer - TaKaRa \\
\hline
\end{tabular}

Note: All primer sequences are listed as 5' to 3'.

miRNA-210 expression analysis was performed in monolayer and high-density pellets during low oxygen cultures in chondrogenic differentiation and functional experiments. For baseline gene expression values, lysates were obtained from expanded cells in monolayer immediately prior to plating cells in pellet culture on day 0 (D0: plating day). Subsequent samples were harvested at D1, D3, and D7 e. Isolated RNA was reverse transcribed into cDNA using MiR-X miRNA FirstStrand Synthesis (TaKaRa, Shiga, Japan). Real-time qPCR was performed using a CFX384 Real-Time System (BioRad, Hercules, CA). miR-21-5p and miR-191-5p were included as reference genes. MiR-210-5p was normalized to miR-21-5p because it showed the lowest variability. Primer sequences are listed in Table 5.1 . 
Table 5.2 Primers sequences used for gene expression analysis.

\begin{tabular}{|lll|}
\hline Gene ID & Forward Primer Sequence & Reverse Primer Sequence \\
\hline ACAN & GTGCCTATCAGGACAAGTCT & GATGCCTTCACCACGACTTC \\
ACTB & AGCACAGAGCCTCGCCTTT & CGGCGATACATCATCCAT \\
AKT1 & ATGGCGCTGAGATTGTGTCA & CCCGGTACACCACGTTCTTC \\
CCNB2 & CCGACGGTGTCCAGTGATTT & TGTTGTTTGGTGGGTTGAACT \\
COL10A1 & CCAGCACGCAGAATCCATC & ATGCCTGTGGGCATTTGGTA \\
COL1A1 & GTAACAGCGGTGACCTGG & CCTCGCTTTCCTTCCTCTCC \\
COL2A1 & GGGATCGTGGTGACAAAGGT & CTGGGCAGCAAAGTTTCCAC \\
GAPDH & ATGTTCGTCATGGGTGTGAA & TGTGGTCATGAGTCCTTCCA \\
HAPLN1 & TCTGGTGCTGATTTCAATCTGC & TGCTTGGATGTGAATAGCTCTG \\
HIF1A & TTCCTTCTCTTCTCCGCGTG & ACTTATCTTTTCTTGTCGTTCGC \\
MKI67 & ACGCCTGGTTACTATCAAAAGG & CAGACCCATTTACTTGTGTTGGA \\
\hline
\end{tabular}

Note: All primer sequences are listed as $5^{\prime}$ to $3^{\prime}$.

mRNA gene expression analysis was performed at distinct time points during chondrogenic differentiation. RNA lysates were obtained prior to plating cells in high-density pellet culture on day 0 and after 7 days of culture in high-density pellet cultures. $1 \mu \mathrm{g}$ of isolated RNA was used as a template for reverse transcription using M-MLV Reverse Transcriptase (Promega, Madison, USA). Gene expression was quantified using quantitative real-time reverse transcriptase polymerase chain reaction (qRT-PCR) with 10 ng cDNA per $10 \mu \mathrm{L}$ with QuantiTect SYBR Green PCR Kit (Qiagen) and the CFX384 Real-Time System (BioRad, Hercules, CA). Relative transcript abundance of genes of interest was

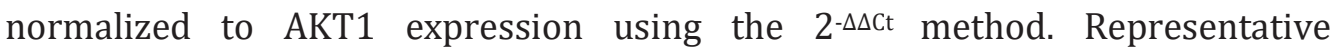
chondrogenic genes (e.g COL2A1, COL1A1, HAPLN1, ACAN and COL10A1), proliferative markers (e.g CYCLIN2A and HIST2H4), hypoxia inducible genes (e.g. HIF1A and HIF3A) and housekeeping genes (e.g. GAPDH, ACTB and AKT1) described in Table 5.2 were used. All values are represented as relative transcript compared to AKT1 (housekeeping gene) \pm standard error of the mean. Three biological replicates $(\mathrm{n}=3)$ and three technical replicate was used.

\subsubsection{Western Blotting}

Total protein was harvested from all different cells 72 hours after transfection. Five cell pellets were pooled from each condition to obtain sufficient protein 
yield. Pooled pellets from each time point were washed twice with ice cold PBS and mechanically lysed using an 18-gauge needle in the presence of radioimmunoprecipitation buffer $(150 \mathrm{mM} \mathrm{NaCl}, 50 \mathrm{mM}$ Tris $\mathrm{pH} 7.4,1 \% \mathrm{w} / \mathrm{v}$ sodium deoxycholate, $0.1 \% \mathrm{w} / \mathrm{v}$ sodium dodecyl sulfate and $1 \% \mathrm{v} / \mathrm{v}$ Triton X100) supplemented with protease inhibitor cocktail (Sigma) and PMSF (Sigma), and stored at $-80^{\circ} \mathrm{C}$ until quantification. Protein was quantified using the $D C^{\mathrm{TM}}$ protein assay (Bio-Rad) according to the manufacturer protocol. Western blotting and membrane development was performed as previously described [8] and the following primary antibodies were used to detect proteins: mouse monoclonal anti-HIF1A (1:500; 610959, BD Biosciences), mouse monoclonal anti-COL2A1 (1:500; MAB8887; Millipore) and rabbit monoclonal anti-GAPDH (1:1000; D16H11; Cell Signaling).

\subsubsection{Histological Analysis}

Cell pellets were collected after 21 days of culture and fixed overnight in 10\% neutral buffered formalin. Samples were then washed and dehydrated in graded series of ethanol (70\% to $100 \%)$ and processed with xylene (50\% to $100 \%)$ prior to paraffin embedding. Paraffin blocks were cut into consecutive sections of $5 \mu \mathrm{m}$ thickness using a microtome and placed onto charged microscope glass slides for histological staining. Following deparaffinization, sections were stained for glycosaminoglycan content using 0.5\% (wt/vol) Alcian Blue 8GX dye (SigmaAldrich) and counterstained with Nuclear Fast Red (Sigma-Aldrich). Collagen type I and collagen type II immunohistochemistry (IHC) was also performed. After removal of paraffin and rehydration, sections were washed with distilled water. Antigen retrieval was performed using $1 \mathrm{mg} / \mathrm{mL}$ pronase for 30 minutes at $37^{\circ} \mathrm{C}$ followed by $10 \mathrm{mg} / \mathrm{mL}$ hyaluronidase for 30 minutes at $37^{\circ} \mathrm{C}$, both in a humidified chamber. Briefly, protein block was applied using 5\% Bovine serum albumin (BSA) in PBS after which slides were incubated overnight with $1 \mu \mathrm{g} / \mathrm{mL}$ of anti-COL2A1 mouse monoclonal Antibody (Millipore, Darmstadt, Germany), 
anti-HIF1A mouse monoclonal Antibody (BD Biosciences, Osage, IA) or antimouse IgG monoclonal Antibody (Abcam) as a control at $4^{\circ} \mathrm{C}$ in a humidified chamber. Slides were sequentially incubated with biotinylated goat anti-mouse IgG HRP secondary antibody and were then treated with 3,3'-diaminobenzidine (DAB) enhanced liquid substrate system for IHC (Sigma-Aldrich) for 10 minutes until color development. Sections were subsequently analyzed using a ZEISS Axio inverted light microscope.

\subsubsection{Statistical Analysis}

Quantitative data for gene expression profiles have been presented as mean \pm standard deviation from 3 independent MSCs donors for each experimental condition and time point. Statistical analysis was performed using JMP® Pro v10.0.0 (SAS Institute), and $p$-values were determined using Tukey HSD test. Significance is noted in the figures when applicable as * for $p<0.05$ and ${ }^{* *}$ for $p$ $<0.01$.

\subsection{RESULTS}

\subsection{1 miR-210 is the distinctive hypoxia induced microRNA in MSCs.}

To evaluate and identify potential miRNAs that are regulated in hypoxia, three different bMSCs and three different aMSCs donors were cultured for one week in monolayer cultures and different oxygen environments: normoxia (20\% oxygen) or hypoxia ( $2 \%$ oxygen). MicroRNAseq analysis was previously performed on the samples (Chapter 4). From section 4.3 .7 only 2 microRNAs were commonly regulated in both MSCs: miR-210 and miR-195. However, the only robustly induced microRNA was miR-210. In bMSCs, miR-210 was induced more than 50fold in hypoxia when compared to normoxia (Figure 4.1.B), whereas in aMSCs fold induction was around 25-fold (Figure 4.6.A). Subsequently, both cells were cultured in monolayer or high-density pellets for 7 days in normoxia or hypoxia. After this time, RNA was extracted and gene expression was analyzed using RTqPCR. As shown in Figure 5.1.A, miR-210 is induced by hypoxia in both cultures. 
Fold-induction for both cells was significantly higher for high-density cultures than in monolayer cultures possibly due to an exaggerated hypoxic effect in the pellet cultures.
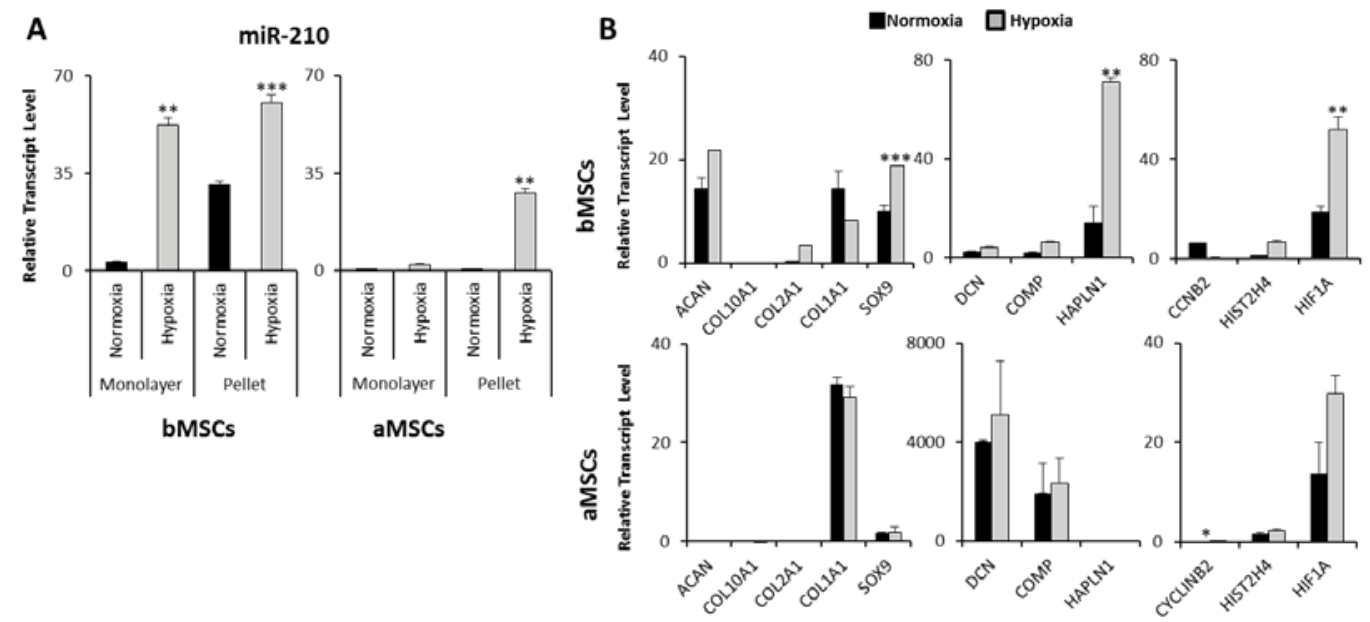

Figure 5.1 (A) miR-210 is highly induced in hypoxia in both MSCs during pellet or monolayer cultures. (B) High-density pellets were used to estimate chondrogenic potential of both MSCs after

7 days in monolayer cultures. Values are represented as relative transcript compared to AKT1 (housekeeping gene) \pm standard error of the mean. Three biological replicates $(n=3)$ and three technical replicate were used. Statistical analysis is represented as * for $p<0.05$, $* *$ for $p<0.01$ and $* * *$ for $p<0.001$.

High-density cultures were used to estimate the chondrogenic potential of both types of MSCs. After 7 days of culture, gene expression of chondrogenic markers (e.g. ACAN, COL2A1, DCN, etc), proliferation markers (e.g. CYCLINB2 and HIST2H4) and hypoxia induced HIF1A were examined (Figure 5.1.B). Chondrogenic markers COL2A1 and ACAN were found to be up-regulated in hypoxia when compared to normoxia in bMSCs. However, expression levels in aMSCS of both chondrogenic markers were unnoticeable. Interestingly, in both MSCs the relative expression of master chondrogenic transcription factor SOX9 was increased in hypoxia. Yet, the expression was significantly up-regulated in bMSCs whereas in aMSCs the expression was low. In contrast, the induction of 
expression of various proteoglycan markers such as DCN and COMP was not celltype dependent. However, chondrogenic marker HAPLN1 was only expressed in both normoxia and hypoxia in bMSCs. Moreover, proliferation marker HIST2H4 was increased when cells were cultured in hypoxia compared to normoxia. Lastly, hypoxia inducible factor HIF1A regulation was similarly regulated in both MSCs. Thus, hypoxia enhances expression of miR-210 irrespective of cell type and upregulates the expression of various chondrogenic markers. The latter clearly showed some cell type specific regulation particularly in the fold change of the response.
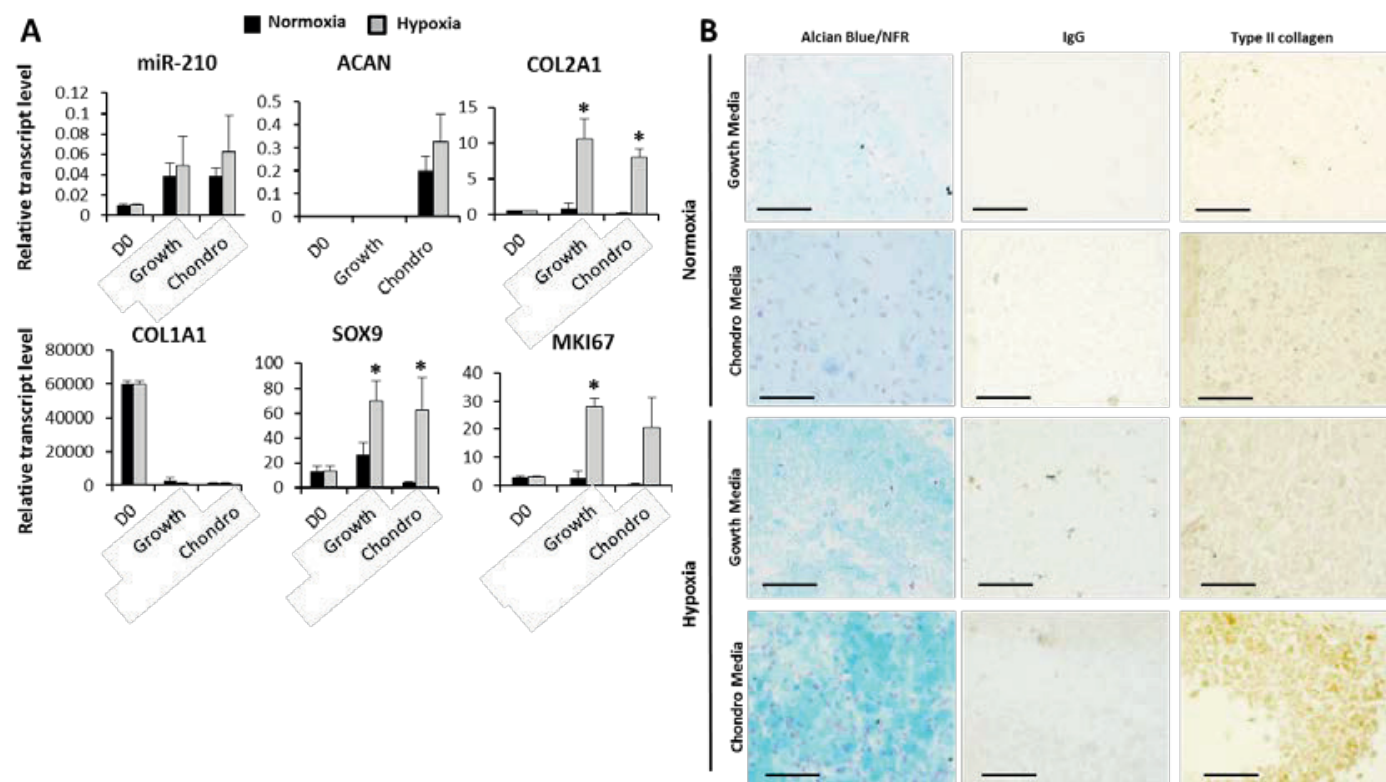

Figure 5.2. (A) RT-qPCR analysis of bMSCs cultured in normoxia and hypoxia. Day 7 chondrogenic differentiation and growth media cultured cells. Values are represented as relative transcript compared to AKT1 (housekeeping gene) \pm standard error of the mean. Three biological replicates $(\mathrm{n}=3)$ and three technical replicates were used. Statistical analysis is represented as * for $p<0.05, * *$ for $p<0.01$ and ${ }^{* * *}$ for $p<0.001$. (B) Histological analysis of MSC grown in maintenance or chondrogenic media and subjected to normoxia and hypoxia conditions. Alcian Blue and Nuclear fast red was used for glycosaminoglycan staining whereas immunohistochemistry was used to obtain specific collagen II staining. (Scale bar $=100 \mu \mathrm{m}$ ). 


\subsubsection{Hypoxia promotes chondrogenic potential of bMSCs and endogenous expression of miR-210.}

We next determined whether miR-210 induction by hypoxia was affected by culturing cells in growth or differentiation media. As observed in Figure 5.2.A endogenous miR-210 expression is induced in a similar manner in both growth and differentiation media. Chondrogenic markers COL2A1 and SOX9 were also induced in both growth and differentiation media but only in hypoxic conditions. Also, proliferation marker MKI67 was significantly induced in hypoxia. However, $A C A N$ was only induced in chondrogenic differentiation media and enhanced in low oxygen cultures. In contrast, collagen type I (COL1A1) was significantly down regulated in hypoxia when cultured in either medium. Figure 5.2.B shows histological staining of bMSCs cultured during 21 days. Alcian blue and COL2A1 specific staining displayed that chondrogenic media and hypoxic cultures increase the chondrogenic potential of bMSCs. Hence, the most abundant proteoglycan deposition and cartilaginous marker expression was found in cells cultured in the chondrogenic differentiation cocktail in low oxygen conditions.

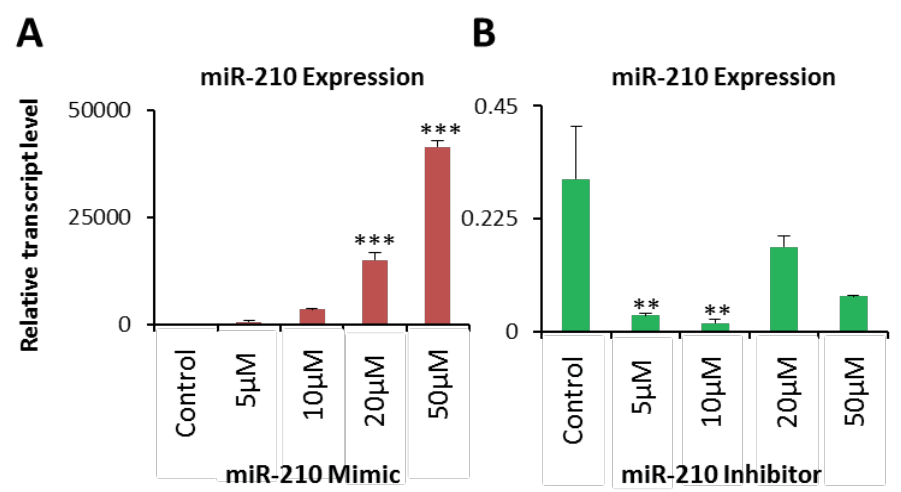

Figure S.5.2. RT-qPCR analysis depicts miR-210 expression of bMSCs transfected with miR-210 mimic (A) or inhibitor (B) at day 3 after transfection. Values are represented as relative transcript compared to AKT1 (housekeeping gene) \pm standard error of the mean. Three biological replicates $(\mathrm{n}=3)$ and three technical replicates were used. Statistical analysis is represented as * for $p<0.05, * *$ for $p<0.01$ and $^{* * *}$ for $p<0.001$. 


\subsection{3 miR-210 overexpression enhances expression of chondrogenic markers in a normoxic environment.}

To assess the role of miR-210 in chondrogenic differentiation of bMSCs, a gain of function analysis was performed in cells cultured in normoxia. First, a dose response experiment was performed to identify the correct stimulatory dose of the miR-210 mimic. Different doses from $5 \mu \mathrm{M}$ to $50 \mu \mathrm{M}$ were utilized and compared (Figure S.5.2). Functional experiments were performed using $20 \mu \mathrm{M}$ of

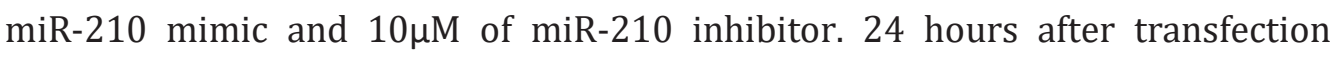
bMSCs were cultured in high-density pellets for up to 21 days. Gene expression analysis was completed during day 1, day 3 and day 7 after transfection for miRNA analysis and day 7 for mRNA analysis.

As observed in Figure 5.3.A, miR-210 expression was highly induced by the mimic when compared to negative control during all time-course. A 100-fold upregulation on day 1 and day 3 was observed. Moreover, miR-210 mimic enhanced standard cartilaginous makers at day 7. COL2A1, ACAN and HAPLN1 increased following the induction of miR-210 while COL1A1 expression was downregulated (Figure 5.3.B). COL2A1/COL1A1 ratio suggested the importance of miR-210 and the presence for collagen type II expression (Figure 5.3.C.) Additionally, histological analysis performed at day 21 after transfection, shows an increase in proteoglycan deposition observed by Alcian blue staining in Figure 5.3.D, and collagen type II enhanced staining in cells transfected with the miR-210 mimic whereas collagen type I expression was decreased (Figure 5.3.E). Moreover, a regulatory feedback loop was observed between HIF1A and miR-210. An enhanced expression of miR-210 promoted the expression of hypoxia inducible factor HIF1A at the mRNA and protein level (Figure 5.3.F). Thus, miR-210 enhances standard cartilaginous markers in bMSCs cultured under regular oxygen conditions and positively regulated HIF1A expression. 
A

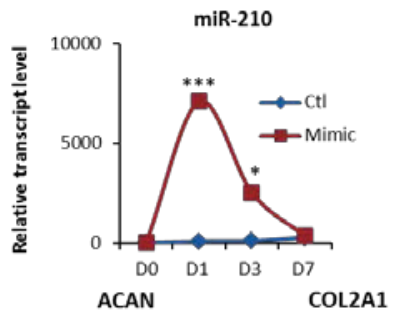

B

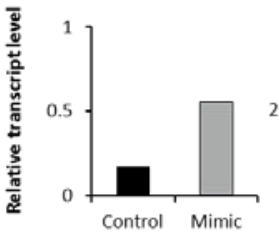

HAPLN1
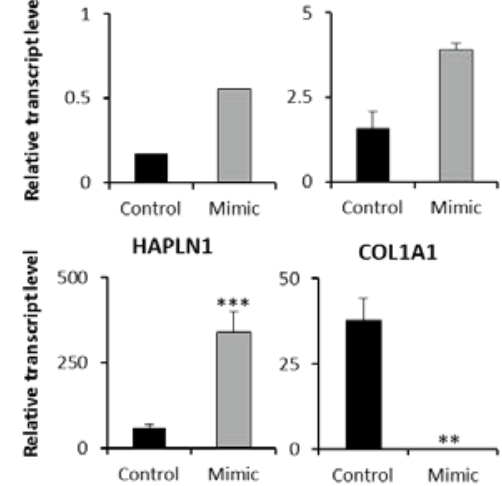

COL1A1

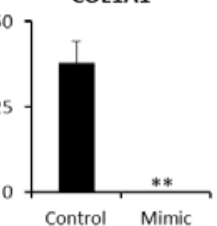

COL2A1/COL1A1

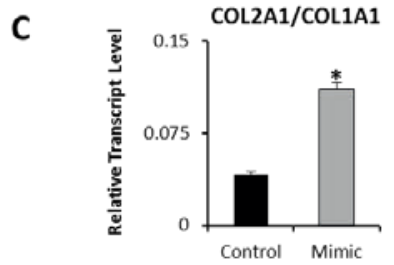

D
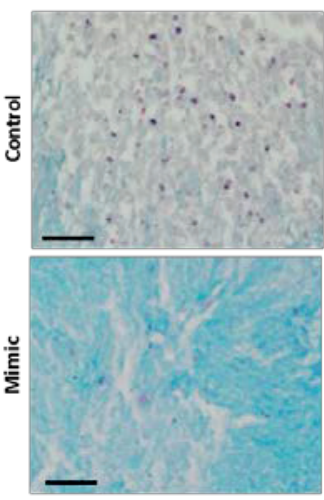

E

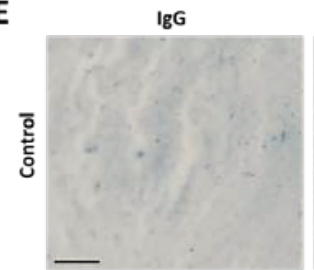

IgG

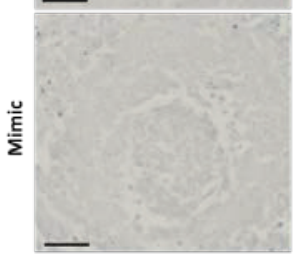

F
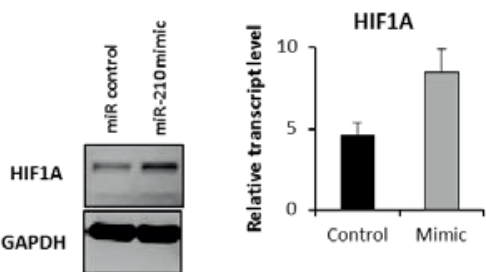

GAPDH

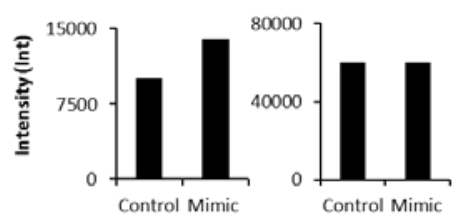

Type I Collagen

Type II Collagen

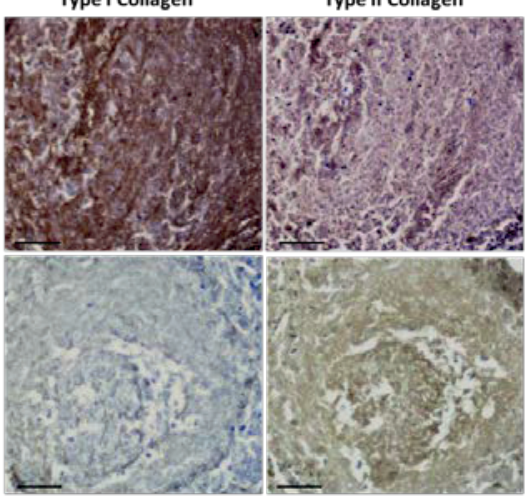

Figure 5.3. Gain of function analysis was performed on bMSCs at day 7. (A) miR-210 was upregulated throughout the time course of the experiment. (B) Chondrogenic markers were enhanced by miR-210 exogenous expression whereas COL1A1 decreased. (C) COL2A1/COL1A1 ratio demonstrated the importance of miR-210 for promoting COL2A1 expression. Values are represented as relative transcript compared to AKT1 (housekeeping gene) \pm standard error of the mean. Three biological replicates $(n=3)$ and three technical replicate were used. Statistical analysis is represented as * for $p<0.05$, ** for $p<0.01$ and ${ }^{* * *}$ for $p<0.001$. (D and E) Histological analysis revealed enhanced proteoglycan deposition and collagen type II synthesis in cells transfected with miR-210 whilst collagen type I synthesis decreased. (Scale bar $=100 \mu \mathrm{m}$ ). (F) Hypoxia inducible factor HIF1A was also induced in a miR-210 dependent manner at the mRNA and protein level. 


\subsection{4 miR-210 inhibition decreases chondrogenic differentiation of MSCs.}

We next performed loss of function experiments of miR 210 in bMSCs cultured in hypoxia. After dose response experiments, $10 \mu \mathrm{M}$ of miR-210 inhibitor was selected as the maximal stimulatory dose (see Figure S.5.2.B). Loss of function experiments was performed by transfecting cells in monolayer cultures after hypoxic exposure. After 24 hours, high-density pellets were formed as previously described. MiRNA gene expression analysis was completed on day 1, day 3 and day 7 after transfection whereas mRNA expression analysis was performed at day 7 after transfection. Figure 5.4.A shows miR-210 expression increased throughout the time course in cells transfected with the negative control. In contrast, cells transfected with the miR-210 inhibitor had significantly lower expression of miR-210 (17-fold decrease at day 7). After miR-210 inhibition was confirmed, expression of chondrogenic markers was evaluated at day 7. As observed in Figure 5.4.B, chondrogenic markers COL2A1, ACAN and HAPLN1 decreased in cells transfected with miR-210 inhibitor, suggesting a potentiating role of miR-210 on the expression of these genes.

Contrarily, COL1A1 expression was enhanced 4-fold in cells transfected with inhibitor compared to negative control miRNA dramatically impacting the COL2A1/COL1A1 ratio (Figure 5.4.C). Histological analysis shows that inhibition of endogenous miR-210 expression prevents proteoglycan deposition and collagen types II and I protein synthesis (Figure 5.4.D and E) pointing to discordance between the inhibitory effect on COL1A1 gene expression and protein expression. In addition, HIF1A was slightly increased at the mRNA and protein level when miR-210 activity was blocked, suggesting a negative regulatory loop between HIF1A and miR-210 (Figure 5.4.F). Consequently, gain and loss of functions experiments demonstrated the relevance of miR-210 activity for chondrogenic potential of MSCs. 
A
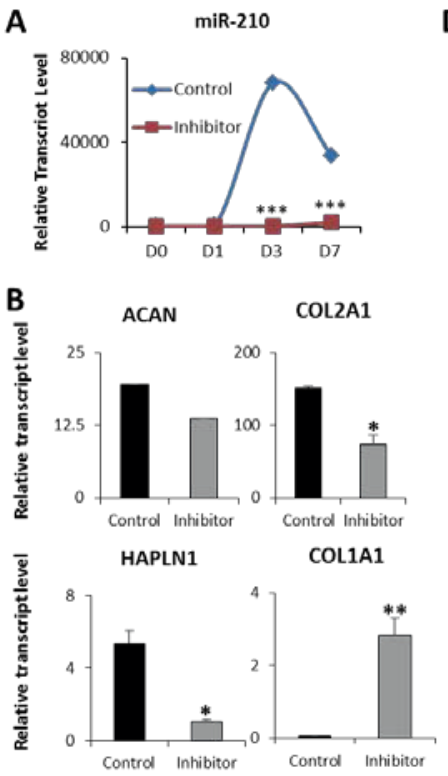

C

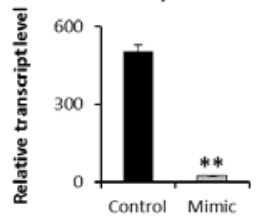

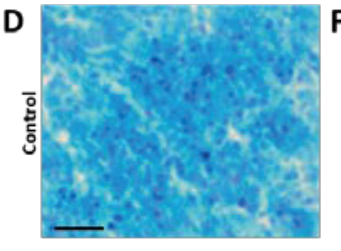
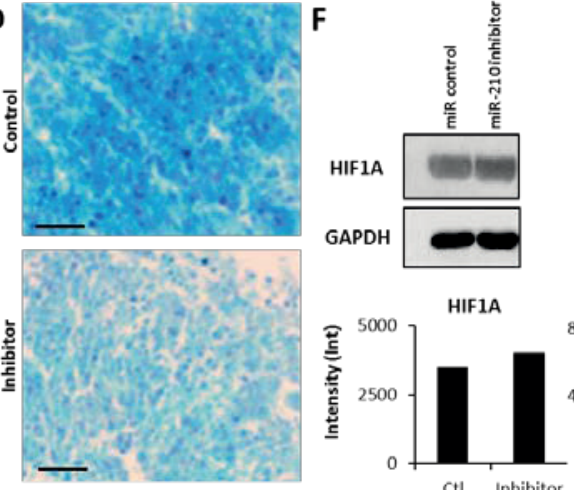

I collagen

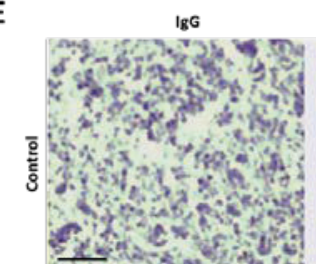

$\lg G$
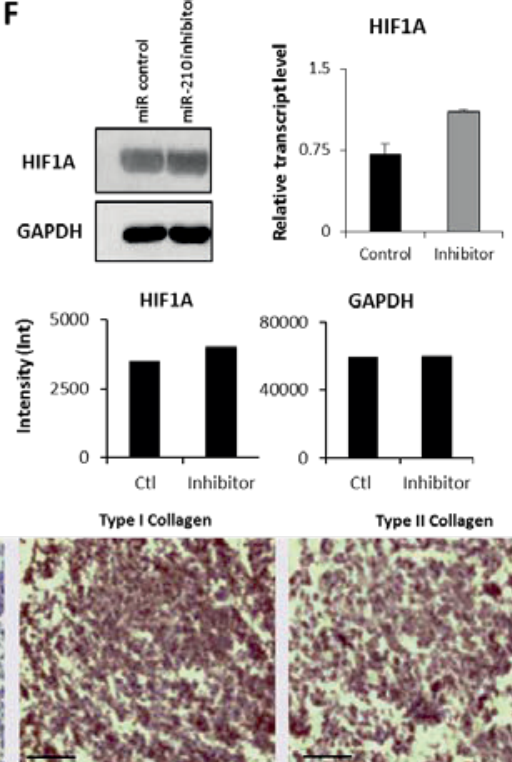

GAPDH

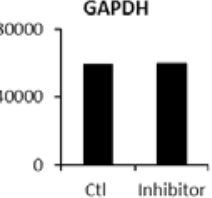

Type II Collagen
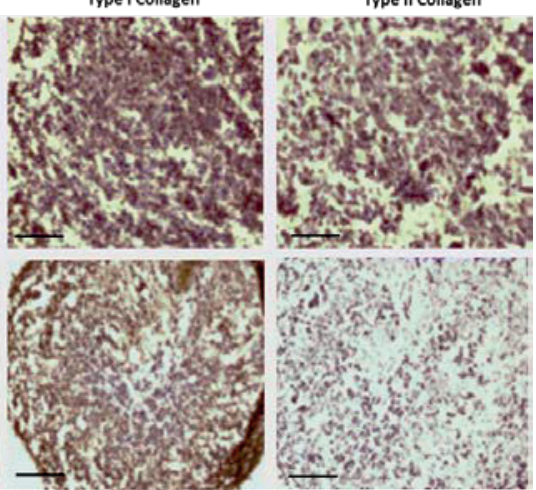

Figure 5.4. Loss of function experiments were performed by transfecting cells in monolayer cultures after hypoxic exposure. (A) miR-210 expression increased throughout the time course in cells transfected with the negative control miRNA whereas cells transfected with the miR-210 inhibitor had significantly lower expression of miR-210. (B and C) Chondrogenic markers COL2A1, ACAN and HAPLN1 decreased in cells transfected with miR-210 inhibitor whilst COL1A1 expression

was up-regulated at day 7. Values are represented as relative transcript compared to AKT1

(housekeeping gene) \pm standard error of the mean. Three biological replicates $(n=3)$ and three technical replicates were used. Statistical analysis is represented as * for $p<0.05$, ${ }^{* *}$ for $p<0.01$ and

$* * *$ for $p<0.001$. (D and E) Histological analysis shows that inhibition of endogenous miR-210 expression prevents proteoglycan deposition and collagen types II and I synthesis. (Scale bar = 100 $\mu \mathrm{m})$. (F) HIF1A was slightly increased at the mRNA and protein levels when miR-210 activity was blocked.

Previous results suggested that miR-210 is an important hypoxia regulatory factor promoting chondrogenesis of bMSCs in culture by increasing the 
COL2A1/COL1A1 mRNA ratio and proteoglycan deposition. Interestingly, upregulation of miR-210 appears sufficient to stimulate the expression of certain cartilage markers, such as COL2A1 and ACAN.

\subsection{5 miR-210 and TGF $\beta 1$ interaction restricts chondrogenic differentiation of bMSCs.}

Previously, we have speculated that the TGF $\beta$ signaling pathway is one of the likely targets of miR-210 (Chapter 4). Since TGF $\beta$ is an essential component in the chondrogenic cocktail for induction of chondrocyte differentiation in bMSC cultures, we therefore set out to explore the interaction between miR-210 and TGF $\beta 1$ in more detail. Transfection of cells using miR-210 mimic or inhibitor was applied in monolayer culture, after which high-density pellets were formed. Throughout the time course cells were maintained in chondrogenic differentiation media containing TGF $\beta 1$. For miR-210 overexpression experiments cells were cultured in normoxia while for loss of function cells were cultured in hypoxia. Figure 5.5.A shows miR-210 expression along the time course of the experiments. In normoxia cultures, miR-210 was significantly enhanced in cells transfected with the miR-210 mimic over a one-week time span compared to the negative control miRNA. In adittion, hypoxic cultures showed a decrease in miR-210 expression in cells transfected with the inhibitor compared to negative control miRNA. Chondrogenic differentiation potential was also analyzed. Both COL2A1 and ACAN expression followed miR-210 activity.

In cells transfected with the mimic, both chondrogenic markers increased more than 2-fold whereas in miR-210 inhibited cells they decreased (Figure 5.5.B). In contrast, COL1A1 gene expression increased in the absence of miR-210. Chondrogenically differentiated cells cultured in hypoxia significantly increased the COL2A1/COL1A1 ratio. Remarkably, histological analysis at day 21 suggested a clear interaction of TGF $\beta 1$ and miR-210. The inhibition of miR-210 in hypoxic cultures decreased both COL2A1 and COL1A1 expression (Figure 5.5.C). In 
addition, protein analysis using western blotting at day 3 shows COL2A1 expression follows miR-210 induction or inhibition (Figure 5.6.B and C).

A

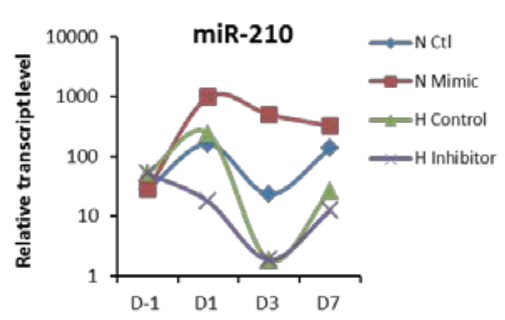

B

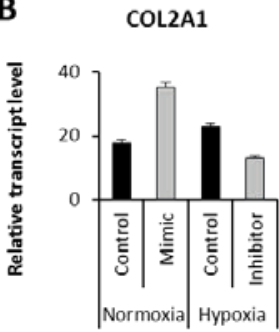

COL1A1

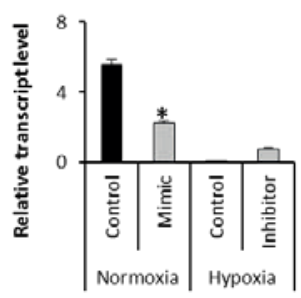

ACAN

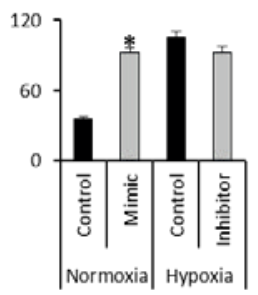

COL2A1/COL1A1

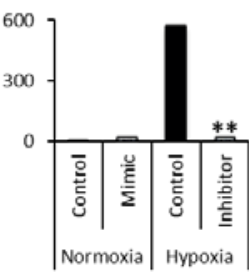

C
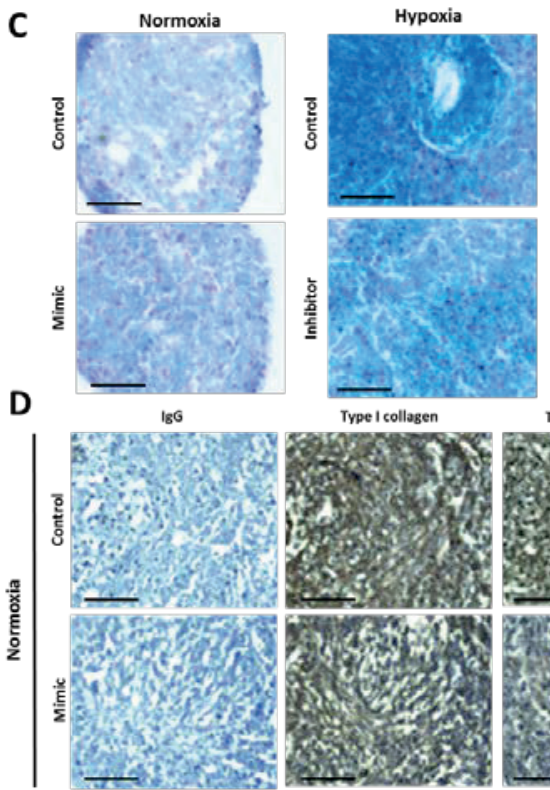

Type I collagen Type II collagen
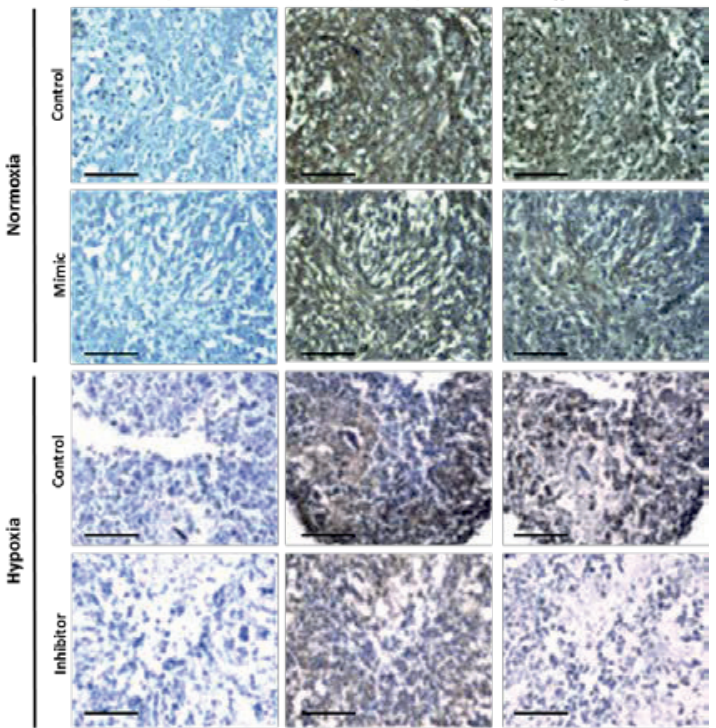

Figure 5.5. (A) miR-210 endogenous expression was overexpressed or inhibited in the presence of chondrogenic differentiation medium containing TGFß1. (B) RT-qPCR analysis showed chondrogenic markers COL2A1 and ACAN followed the expression of miR-210 at day 7. In contrast, COL1A1 expression opposed miR-210 activity. COL2A1/COL1A1 ratio revealed a delayed in chondrogenesis in miR-210 overexpressed MSCs whilst best chondrogenesis was observed in cells cultured in hypoxia and induced with TGF $\beta 1$. Values are represented as relative transcript compared to AKT1 (housekeeping gene) \pm standard error of the mean. Three biological replicates $(\mathrm{n}=3)$ and three technical replicates were used. Statistical analysis is represented as * for $p<0.05, * *$ for $p<0.01$ and ${ }^{* * *}$ for $p<0.001$. (C) Histological analysis exposed a delay in chondrogenesis of MSCs when they are exposed to both miR-210 mimic and TGFß1. (Scale bar $=100 \mu \mathrm{m})$. 
Moreover, HIF1A expression was induced by miR-210 overexpression and blocked during miR-210 inhibition at the mRNA and protein level (Figure 5.6). Comparison between cells cultured in growth media (Figure 5.3.B and C) and cells in chondrogenic media (Figure 5.5.B) showed a better potential of miR-210 mimic to enhance COL2A1/COL1A1 ratio, and proteoglycan and collagen type II deposition in cells expanded in growth media and overexpressing miR-210. For chondrogenesis purposes, hypoxia and miR-210 might be a better regulatory mechanism to enhance cartilaginous markers whilst preventing hypertrophic differentiation on the cells than TGF $\beta 1$.
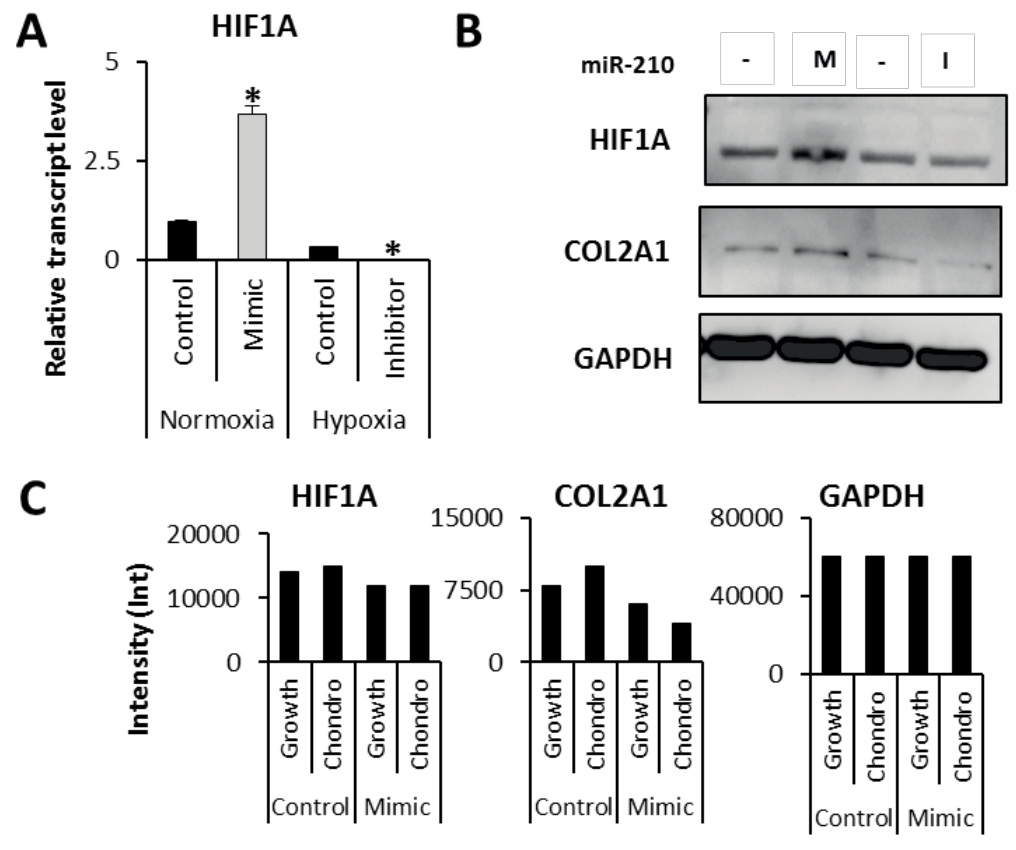

Figure 5.6. (A) RT-qPCR gene expression analysis of $H I F 1 A$ at day 7. Values are represented as relative transcript compared to AKT1 (housekeeping gene) \pm standard error of the mean. Three biological replicates $(n=3)$ and three technical replicate were used. Statistical analysis is represented as * for $p<0.05$, ** for $p<0.01$ and ${ }^{* * *}$ for $p<0.001$. (B and C) HIF1A protein levels were induced or inhibited following miR-210 expression. 


\subsection{6 miR-210 promotes chondrogenesis of bMSCs similarly to TGFß1 in hypoxic conditions, but interaction of both factors increases hypertrophic differentiation of the cells.}

Transfection of miR-210 mimic further increased miR-210 expression in cells cultured in growth media under hypoxic conditions (Figure 5.7.A). Gene expression analysis was performed on standard cartilage markers COL2A1, ACAN and HAPLN1 to analyze chondrogenesis on the cells. Both HAPLN1 and ACAN expression increased in cells transfected with the miR-210 mimic, whereas a slight decrease in COL2A1 was observed (Figure 5.7.B). In addition, miR-210 mimic without the addition of chondrogenic media, significantly increased COL2A1 protein expression at day 3. (Figure 5.7.D). COL1A1 expression decreased in cells transfected with miRNA-210 mimic while it increased in cells containing both mimic and TGF $\beta 1$. The change in COL2A1/COL1A1 ratio exposed how overexpression of miR-210 in cells can promote chondrogenesis of MSCs similarly to the chondrogenic differentiation cocktail. However, miR-210 overexpression seems to enhance collagen type X expression. HIF1A mRNA levels were not regulated by the miR-210 mimic nor by TGF $\beta 1$ induction. In contrast, the addition of chondrogenic media increased HIF1A protein content but this expression decreased when miR-210 mimic was added (Figure 5.7.C and D). Therefore, miR-210 promotes chondrogenesis on bMSCs in a similar way as cells cultured in chondrogenic differentiation cocktail and hypoxia. The addition of chondrogenic cocktail to cells overexpressing miR-210 decreases the synthesis and deposition of important chondrogenic proteins such as ACAN and HAPLN1 while increasing fibroblastic marker collagen type I, suggesting that the beneficial effects of miR-210 are dose-dependent. 


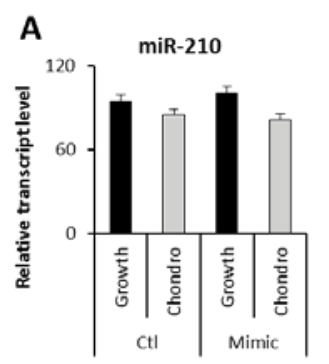

B $\quad$ COL2A1

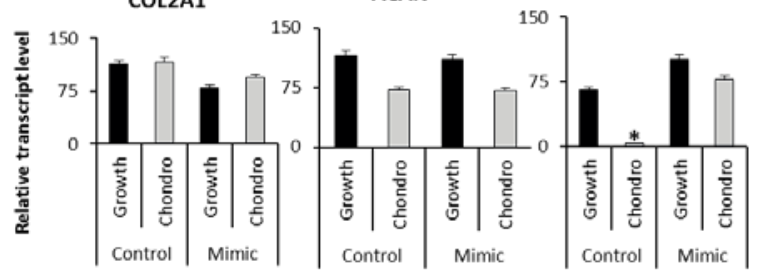

COL1A1

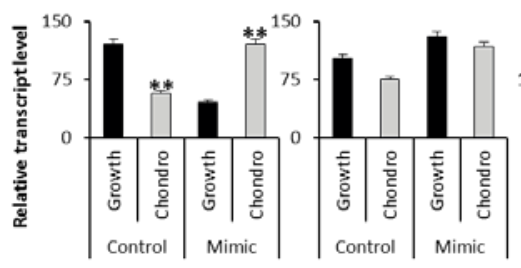

COL2A1/COL1A1

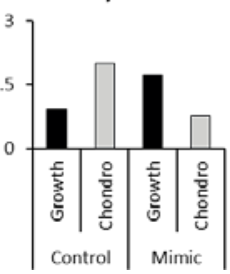

D

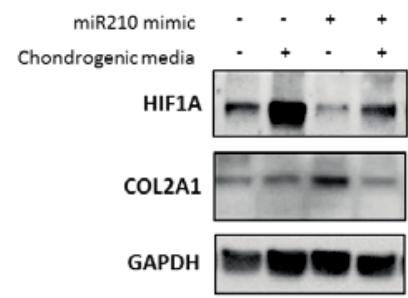

HIF1A

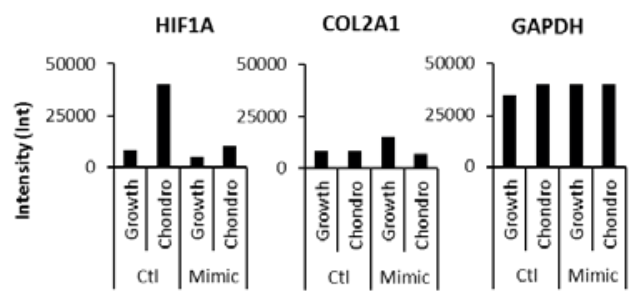

Figure 5.7. Gene expression analysis was performed 7 days after transfection. (A) miR-210 expression was overexpressed in the presence of growth media or chondrogenic differentiation media containing TGF $\beta 1$ in hypoxia. (B) Both HAPLN1 and ACAN decreased in cells transfected with miR-210 mimic whereas a slight decrease in COL2A1 was observed in chondrogenic-differentiated cells. Interestingly, COL1A1 expression decreased in chondrogenic differentiated cells transfected with negative control miRNA while it increased in cells containing both mimic and TGF $\beta 1$. Collagen

type $\mathrm{X}$ increased with the addition of miR-210 mimic. COL2A1/COL1A1 ratio exposed how overexpression of miR-210 in cells can promote chondrogenesis of MSCs similarly to the chondrogenic differentiation cocktail. Values are represented as relative transcript compared to AKT1 (housekeeping gene) \pm standard error of the mean. Three biological replicates $(\mathrm{n}=3)$ and three technical replicates were used. Statistical analysis is represented as * for $p<0.05$, ${ }^{* *}$ for $p$ $<0.01$ and $* * *$ for $p<0.001$. (C and D) HIF1A mRNA levels were not regulated by the miR-210 mimic nor TGF $\beta 1$ induction. In contrast, the addition of chondrogenic media increased HIF1A protein content but this expression decreased when miR-210 exogenous expression increased. 


\subsection{DISCUSSION}

Formation of cartilaginous tissues with sufficient collagen type II and proteoglycans is essential to reconstruct cartilage with restored functions. However, this is still difficult to accomplish with current tissue engineering methods. Engineered cartilage using MSCs may become hypertropic resulting in the production of bone-like or fibrotic tissues once implanted [9]. The physiological conditions in the articular cartilage are very complex, consisting of low oxygen environment and repeated mechanical loading. Pre-programming the cells in the cartilage native physiological conditions may be helpful for enhancing the chondrogenic phenotype while preparing the cells for implantation. Not a lot of literature was found on tissue engineering applications using low oxygen tension. Although some biomaterials used in cartilage regeneration strategies mimic a hypoxic microenvironment for the cells, the cells are still subjected to normal oxygen levels in culture. The equipment required to maintain the cells in a hypoxic microenvironment is often expensive and space restrictive. As a consequence, it would be interesting to perform in vitro chondrogenic differentiation of MSCs using a regulatory mechanism that would mimic the hypoxic response on the cells without the need of low oxygen cultures.

miRNAs are involved in the hypoxic response and contribute to the repression of specific genes under low oxygen conditions [10-13]. Several miRNAs are induced under hypoxic conditions (e.g. miR-210, -21, -23, -24, -26, -103/107, and -181) [14] and have been implicated in various cellular and molecular processes, such as cell growth arrest, stem cell survival, mitochondrial metabolism, DNA repair, angiogenesis and osteoblastic and adipogenic differentiation [15]. MicroRNAseq analysis performed on bMSCs and aMSCs cultured over one week in monolayer and exposed to normoxia or hypoxia conditions, revealed miR-210 as the only robustly inducible miRNA in hypoxia with a fold change of at least 25. miR-210 has been established as the only hypoxic induced miRNA agreed on by all the studies to date [12]. Yet, miR-210 regulation of MSCs chondrogenesis has not 
been proven. When compared directly, the absolute levels of miR-210 induced by hypoxia are largely similar across most cells [7]. However, in our studies, a twofold change difference of miR-210 expression in bMSCs compared to miR-210 expression in aMSCs suggested why bMSCs could be better at promoting chondrogenesis than aMSCs. Remarkably, exposure of cultured cells to low $\mathrm{pH}$, oxidant stress, growth factor deprivation, and osmotic stress to mimic hypoxiaassociated processes does not induce miR-210 expression [16]. To describe the regulatory mechanism of miR-210 during chondrogenesis of bMSCs, its expression was regulated using a miR-210 specific mimic or an inhibitor. miR210 mimic increased expression up to 100 -fold and inhibitor decreased it by 17 fold. This exogenous expression of miR-210 was conserved in culture for at least 1 week. Functional experiments revealed miR-210 importance for early chondrogenesis of MSCs during in vitro cultures. Interestingly, exogenous induction of miR-210 enhanced chondrogenesis of bMSCs as good as TGF $\beta 1$ induction under low oxygen cultures based on the level of expression of various chondrogenic marker genes and (immuno)histological analysis. However, the addition of chondrogenic cocktail to cells overexpressing miR-210 decreases the synthesis and deposition of important chondrogenic proteins, such as ACAN and HAPLN1, while increasing fibroblastic marker collagen type I. A compensatory mechanism seems to keep collagen type I expression in bMSCs in cells containing chondrogenic cocktail, despite the changes in miR-210 and collagen type II levels. In cartilage, TGF $\beta 1$ controls the production of extracellular matrix (ECM) by increasing the synthesis of proteoglycans, collagens and fibronectin. However, it also exerts inflammatory responses and osteophyte formation if present in the joint for too long. Despite the fact that TGF $\beta 1$ increases cellular proliferation and causes earlier and extensive deposition of collagen type II and greater accumulation of proteoglycans, it also induces gene expression of degrading enzymes such as MMPs and collagen type X. An increase in angiogenic markers expression could start a pro-inflammatory response in the cells promoting the 
up-regulation of hypertrophic and early mineralization markers over cartilaginous genes [17].

A

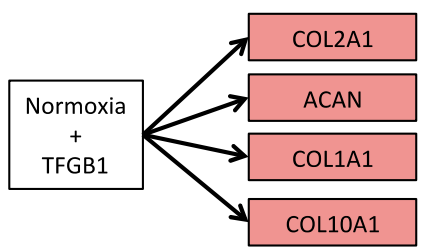

个Hypertrophic cartilage formation
B

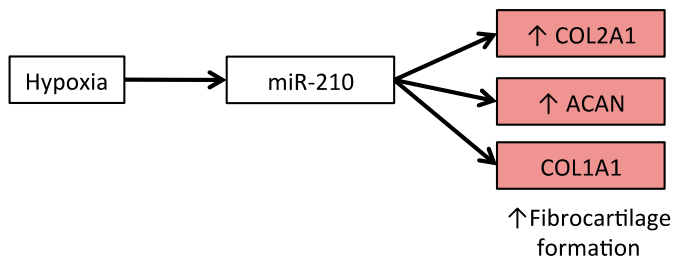

C

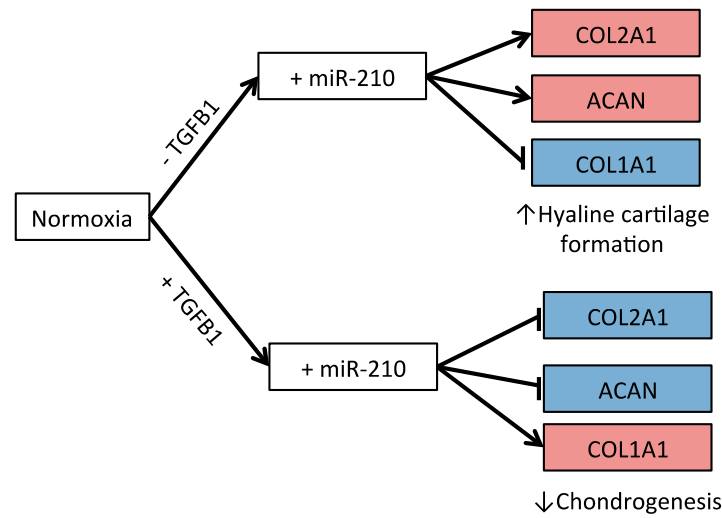

Figure 5.8. Mechanistic model of chondrogenic differentiation via miR-210 regulation in bMSCs.

(A) TGF 1 induces hypertrophic cartilage formation of MSCs in normoxia by increasing standard cartilaginous markers but also promoting hypertrophic markers. (B) Hypoxia induction of endogenous miR-210 programs MSCs to express cartilaginous markers COL2A1 and ACAN but also increases COL1A1, which could result in fibrocartilage formation. (C) Exogenous induction of miR210 using miRNA mimic induces standard cartilaginous markers whilst inhibiting hypertrophic/fibroblastic markers promoting MSCs to form hyaline cartilage. In contrast, induction of exogenous levels of miR-210 in the presence of TGF $\beta 1$ decreases chondrogenesis of MSCs reducing expression of cartilage markers and increasing COL1A1 expression. Up-regulated genes are indicated with red whereas down-regulated genes are indicated in blue.

Additionally, multiple groups have found that miR-210 is specifically induced by HIF1A [16]. Although HIF1A gene expression is primarily triggered by changes in oxygen concentration, it is also modulated by several other pathways, including reactive oxygen species and the phosphatidylinositol 3-kinase (PI3K)/Akt 
pathway [18]. Conclusively, Figure 5.8 describes the mechanistic model of miR210 regulation of chondrogenesis in MSCs. Chondrogenic differentiation cocktail containing TGF $\beta 1$ in standard oxygen cultures enhances chondrogenesis of MSCs evidenced by increased proteoglycan deposition and cartilaginous markers expression. However, it also promotes hypertrophic markers (Figure 5.8.A). Hypoxia induction of endogenous miR-210 programs bMSCs to express cartilaginous markers COL2A1 and ACAN but increases COL1A1, which could result in fibrocartilage formation (Figure 5.8.B). Exogenous induction of miR210 using miRNA mimic induces standard cartilaginous markers whilst inhibiting hypertrophic/fibroblastic markers promoting bMSCs to form hyaline cartilage. In contrast, induction of exogenous levels of miR-210 in the presence of TGFß1 decreases chondrogenesis of bMSCs reducing expression of cartilage markers and increasing COL1A1 expression (Figure 5.8.C).

The major limitation of the present study is the use of serum containing media (growth media) versus the serum-free media with TGF $\beta 1$ (chondrogenic media). Previously, some studies have described the benefits of the presence of FBS for chondrogenesis of bMSCs [19]. However, this chondrogenic potential was inconsistent and varied from batch to batch. In contrast, several studies have described the benefits of serum-free media conditions to enhance and promote higher expression of glycosaminoglycans and collagen type II [20] when compared to serum based cultures. In the present study, we observed how MSCs in the presence of serum (e.g FBS or PL) and under hypoxia expressed SOX9, ACAN and COL2A1. Remarkably, histological analysis showed that a proper chondrogenic induction and collagen type II deposition in MSCs requires the presence of TGF $\beta 1$ and hypoxia. Furthermore, the high chondrogenic potential observed under this culture condition (e.g. hypoxia + TGFß1) was also achieved by overexpression of miR-210 even in cultures containing FBS or platelet lysate (data not shown) and under standard oxygen conditions (e.g. normoxia). Future studies will elucidate if the use of serum-free conditions and miR-210 will further 
induce chondrogenic differentiation of bMSCs more abundantly that under serum containing cultures.

Therefore, miR-210 exogenous induction can be used instead of chondrogenic differentiation cocktail in standard or low oxygen cultures to promote chondrogenesis of bMSCs while inhibiting their hypertrophic differentiation. In this study, we propose that hypoxia stimulated chondrogenesis of MSCs is mainly driven by upregulation of miR-210. From our results, we conclude that miR-210 in combination with hypoxic conditions enhanced in vitro chondrogenesis of bMSCs in comparison with chondrogenic differentiation cocktail containing TGF $\beta 1$ in the cells. Both standard cartilage markers and the COL2A1/COL1A1 ratio was increased when miR-210 mimic was added to the cells. Remarkably, the use of miR-210 mimic does not present negative side effects, such as increase in COL1A1 expression and induction of hypertrophic markers, as compared to TGF $\beta 1$. This potential treatment can help in regeneration of hyaline cartilage preventing formation of fibrocartilage in vivo. 


\section{REFERENCES}

1. Coimbra I, Jimenez S, Hawkins D, Piera-Velazquez S, Stokes D: Hypoxia inducible factor-1 alpha expression in human normal and osteoarthritic chondrocytes. Osteoarthritis Cartilage 2004, 12:336-345.

2. Amarilio R, Viukov S, Sharir A, Eshkar-Oren I, Johnson R, Zelzer E: HIF1 $\alpha$ regulation of Sox9 is necessary to maintain differentiation of hypoxic prechondrogenic cells during early skeletogenesis. Development 2007, 134:3917-3928.

3. Almalki SG, Agrawal DK: Key transcription factors in the differentiation of mesenchymal stem cells. Differentiation 2016, 92:41-51.

4. Caplan AI: MSCs: The Sentinel and Safe-Guards of Injury. J Cell Physiol 2016, 231:1413-1416.

5. $\quad$ Bertero T, Rezzonico R, Pottier N, Mari B: Impact of MicroRNAs in the Cellular Response to Hypoxia. Int Rev Cell Mol Biol 2017, 333:91-158.

6. Leijten J, Georgi N, Moreira Teixeira L, van Blitterswijk CA, Post JN, Karperien M: Metabolic programming of mesenchymal stromal cells by oxygen tension directs chondrogenic cell fate. Proc Natl Acad Sci U S A 2014, 111:13954-13959.

7. Chan YC, Banerjee J, Choi SY, Sen CK: miR-210: the master hypoxamir. Microcirculation 2012, 19:215-223.

8. Dudakovic A, Camilleri E, Riester SM, Lewallen EA, Kvasha S, Chen X, Radel DJ, Anderson JM, Nair AA, Evans JM, van Wijnen AJ: High-resolution molecular validation of self-renewal and spontaneous differentiation in clinical-grade adipose-tissue derived human mesenchymal stem cells. J Cell Biochem 2014, 115:1816-1828.

9. Wu L, Leijten JC, Georgi N, Post JN, van Blitterswijk CA, Karperien M: Trophic effects of mesenchymal stem cells increase chondrocyte proliferation and matrix formation. Tissue Eng Part A 2011, 17:1425-1436.

10. Donker RB, Mouillet JF, Nelson DM, Sadovsky Y: The expression of Argonaute2 and related microRNA biogenesis proteins in normal and hypoxic trophoblasts. Mol Hum Reprod 2007, 13:273-279.

11. Kulshreshtha R, Ferracin M, Wojcik SE, Garzon R, Alder H, Agosto-Perez FJ, Davuluri R, Liu CG, Croce CM, Negrini M: A microRNA signature of hypoxia. Mol Cell Biol 2007, 27:1859-1867.

12. Huang F, Zhu X, Hu XQ, Fang ZF, Tang L, Lu XL, Zhou SH: Mesenchymal stem cells modified with miR-126 release angiogenic factors and activate Notch ligand Delta-like-4, enhancing ischemic angiogenesis and cell survival. Int J Mol Med 2013, 31:484-492.

13. Ivan M, Huang X: miR-210: fine-tuning the hypoxic response. Adv Exp Med Biol 2014, 772:205-227.

14. Yang B, Guo H, Zhang Y, Chen L, Ying D, Dong S: MicroRNA-145 regulates chondrogenic differentiation of mesenchymal stem cells by targeting Sox9. PLoS One 2011, 6:e21679.

15. Georgi N, Taipaleenmaki H, Raiss CC, Groen N, Portalska KJ, van Blitterswijk C, de Boer J, Post JN, van Wijnen AJ, Karperien M: MicroRNA Levels as Prognostic Markers for the Differentiation Potential of Human Mesenchymal Stromal Cell Donors. Stem Cells Dev 2015, 24:1946-1955.

16. Chan SY, Loscalzo J: MicroRNA-210: a unique and pleiotropic hypoxamir. Cell Cycle 2010, 9:1072-1083.

17. Al-Yamani A, Kalamegam G, Ahmed F, Abbas M, Sait KHW, Anfinan N, Al-Wasiyah MK, Huwait EA, Gari M, Al-Qahtani M: Evaluation of in vitro chondrocytic differentiation: 
A stem cell research initiative at the King Abdulaziz University, Kingdom of Saudi Arabia. Bioinformation 2018, 14:53-59.

18. Fahling M: Cellular oxygen sensing, signalling and how to survive translational arrest in hypoxia. Acta Physiol (Oxf) 2009, 195:205-230.

19. Solchaga LA, Penick K, Porter JD, Goldberg VM, Caplan AI, Welter JF: FGF-2 enhances the mitotic and chondrogenic potentials of human adult bone marrow-derived mesenchymal stem cells. J Cell Physiol 2005, 203:398-409.

20. Cho H, Lee A, Kim K: The effect of serum types on Chondrogenic differentiation of adipose-derived stem cells. Biomater Res 2018, 22:6.

21. Galeano-Garces C, Camilleri ET, Riester SM, Dudakovic A, Larson DR, Qu W, Smith J, Dietz AB, Im HJ, Krych AJ, Karperien M, van Wijnen AJ: Molecular Validation of Chondrogenic Differentiation and Hypoxia Responsiveness of Platelet-Lysate Expanded Adipose Tissue-Derived Human Mesenchymal Stromal Cells. Cartilage 2017, 8:283-299. 


\section{CHAPTER 6}

\section{Human synovial fluid assists human primary chondrocytes and mesenchymal stem cells chondrogenic differentiation during culture expansion.}




\section{ABSTRACT}

Synovial fluid (SF) is a biological fluid that covers the synovial joints and provides metabolic supply to the chondrocytes in the native cartilage tissue. Moreover, it maintains lubrication and shock absorption properties due to its mechanical properties. The lubrication and elasticity of this tissue is derived from its rich composition in hyaluronan and lubricin. This fluid is highly examined to study pathophysiological changes during osteoarthritis. Our purpose was to determine the usability of synovial fluid as a human based product for in vitro expansion, culture and chondrogenic re-differentiation of human chondrocytes and stem cells for clinical applications in cartilage regeneration. Metabolic activity assays on primary chondrocytes and adiposederived stromal stem cells (aMSCs), showed both cell types survived and proliferated during culture with synovial fluid. However, different effects were observed. aMSC preferred SF samples with higher protein content whereas primary chondrocyte viability was increased with lower protein content samples. Primary chondrocyte proteoglycan deposition was maintained in cell cultures containing synovial fluid. Synovial fluid seems to promote chondrogenic differentiation of aMSC in the presence of chondrogenic differentiation medium, which was confirmed by positive collagen type II immunohistochemistry. Our results serve as an initial screening of the possibilities of SF to replace fetal bovine serum as a culture supplement for in vitro expansion of human primary chondrocytes and aMSCs. Thus, synovial fluid is a promising non-zoonotic and animal free alternative for culture expansion of chondrocytes and stem cells for cartilage repair strategies. 


\subsection{INTRODUCTION}

The development of cell-based therapies to improve cartilage repair has received considerable interest in recent years. Cells need exposure to a wide variety of stimuli to mimic in vivo conditions. Attempts to identify the required stimuli to drive the formation of permanent articular cartilage have been largely unsuccessful to date. Synovial fluid (SF) is a viscous, mucinous biological fluid that covers the synovial joints and it's located between the articular cartilage and the synovial membrane [1]. Due to the avascular nature of cartilage, this tissue does not obtain its metabolic supply from the blood vessels but from the synovial fluid. Thus, its principal function is to provide enough metabolic supply (e.g. oxygen and nutrients) to the chondrocytes in the cartilage. In physiological conditions during movement, synovial fluid maintains the lubrication of the cartilage surface in articular joints, allowing bones to freely articulate by friction reduction and shock absorption [2]. The mechanical properties of the synovial fluid are derived from the actively secreted substances: hyaluronic acid, lubricin, proteinases, collagenases and prostaglandins that lubricate and increase the elasticity in the joint. Although chondrocytes in cartilage constantly produce hyaluronan and proteoglycans, they produce it at a low level [3]. Hyaluronan is one of the principal components of SF $[4,5]$.

Currently, various clinical trials inject stem cells into the knee joint with the hope of promoting healing of osteochondral defects and osteoarthritis [6]. For stem cell therapies, the study of the interaction of stem cells with the joint microenvironment is essential to understand cell viability and differentiation potential in physiological conditions. Moreover, is important to determine if synovial fluid exerts negative or positive effects on these cells. Due to its location and the ease of collection [7], synovial fluid has been examined to study changes in the joint microenvironment [8]. However, there are no studies about the effects of the synovial fluid to the injected stem cells. The purpose of this study aimed to determine the usability of synovial fluid as a human derived product for 170 
in vitro expansion, culture and differentiation of human chondrocytes and human adipose-derived stem cells for clinical applications.

\subsection{MATERIALS AND METHODS}

\subsubsection{Collection of fluid.}

Synovial fluid specimens were obtained from patients undergoing knee replacement surgery or knee drainage as therapy for flare-ups of joint disease, with approval from the Mayo Clinic Institutional Review Board (IRB) (IRB \#14000440). Synovial fluid was collected using knee aspiration. In total synovial fluid of 54 patients was collected and analyzed in this study. For all donors, the stage of disease was classified in accordance with the Kellgren and Lawrence classification system of knee osteoarthritis. Grading was determined by visible cartilage lesions seen with radiographic imaging and was grouped into healthy (KL 0), mild (KL grade 1-2), moderate (KL grade 3 ) and severe (KL grade 4). After biological samples were collected in the procedure room, they were centrifuged at $12000 \mathrm{rpm}$ for 10 minutes and the supernatant was aliquoted into sterile tubes. All samples were snap frozen and stored at $-80^{\circ} \mathrm{C}$ until analysis.

\subsubsection{Specimen analysis.}

Physical and chemical evaluation was performed on the samples including the recording of the aspirated volume, the color and clarity. Synovial fluid samples were classified as previously described based on their appearance (color and clarity) [9]. For chemical examination protein concentration was determined using DC ${ }^{\mathrm{TM}}$ Protein Assay (Biorad, Hercules, USA) following the manufacturer's instructions.

\subsubsection{Cell harvest and monolayer culture.}

Two different cell types were used for these studies. Adipose derived mesenchymal stem cells (aMSCs) were obtained from lipo-aspirates from three representative healthy donors as described by [10]. Human primary 
chondrocytes were obtained from the calcaneal apophysis of consenting donors. Studies with both cell types were approved by the Mayo Clinic Institutional Review Board (IRB). Both cell types were expanded in growth media. Growth media for aMSCs was composed of Advanced MEM (Gibco/Thermo Fisher Scientific, Waltham, MA) supplemented with 5\% human platelet lysate (PL-Max, MillCreek Life Sciences, Rochester, MN), 1\% penicillin/streptomycin, 1\% Glutamax (Gibco/Thermo Fisher Scientific) and 0.2\% heparin (Baxter, Deerfield, IL). Passage 4 human primary chondrocytes were cultured in maintenance medium that contained advanced MEM (Gibco/Thermo Fisher Scientific) supplemented with $10 \%$ fetal bovine serum (FBS, Atlanta Biologicals, Atlanta, GA, Atlanta, GA) and 1\% penicillin/streptomycin (Gibco/Thermo Fisher Scientific). Cells were cultured using standard procedures in T-175 $\mathrm{cm}^{2}$ flasks at $37{ }^{\circ} \mathrm{C}, 95 \%$ humidity and $5 \% \mathrm{CO}_{2}$ until they reached $80 \%$ confluence. Prior to each experiment, cells were detached from T175 flasks by trypsinization using TrypLE Express (Gibco/Thermo Fisher Scientific).

\subsubsection{Proliferation and viability assays}

For viability assays, both aMSCs and chondrocytes were cultured in 96 wellplates at a density of 3,000 cells $/ \mathrm{cm}^{2}$. Proliferating cells were used for analysis. Synovial fluid samples with a protein content of $10 \mu \mathrm{g} / \mu \mathrm{L}$ were used as stock solution for further dilutions. Cells were grown in a volume per volume or twofold dilutions series of the synovial fluid stock sample diluted in culture media. The dilutions used were: $10 \mu \mathrm{g} / \mu \mathrm{l}, 5 \mu \mathrm{g} / \mu \mathrm{l}, 2.5 \mu \mathrm{g} / \mu \mathrm{l}, 1.25 \mu \mathrm{g} / \mu \mathrm{l}, 0.63 \mu \mathrm{g} / \mu \mathrm{l}, 0.3$ $\mu \mathrm{g} / \mu \mathrm{l}$ and $0.15 \mu \mathrm{g} / \mu \mathrm{l}$ or volume per volume dilutions of $1 / 2,1 / 5,1 / 10,1 / 20$, $1 / 40,1 / 80$ and 1/160. Fetal bovine serum (FBS) or platelet lysate were used as a control. Growth media was replaced with advanced MEM media containing different dilutions of SF, 5\% platelet lysate (PL) or 10\% FBS, and cultured for 24hrs. To examine if the PBS content in the diluted samples affects the metabolic activity of chondrocytes, proliferative and confluent cells were analyzed using 
two-fold dilutions of synovial fluid as described before. Metabolic activity was determined using the CellTiter 96® AQueous Non-Radioactive Cell Proliferation Assay (MTS) (Promega, Madison, USA). Optimal culture concentration of synovial fluid for each cell type was deducted from these experiments.

For proliferation rate and growth curve analysis, cells were seeded in 6 wellplates at a density of 5,000 cells $/ \mathrm{cm}^{2}$. For this set of experiments, the 10 most stimulatory and the 10 most inhibitory synovial fluid samples for each cell type were used. Growth media was replaced with advanced MEM media containing $5 \%$ SF, $5 \%$ PL or $10 \%$ FBS, and cultured for 7 days. For cell quantification, live staining was performed using LIVE/DEAD viability/cytotoxicity kit for mammalian cells (ThermoFisher Scientific, Waltham MA, USA). Cell counting was performed each day from day 1 until day 7. Pictures were taken from 3 different parts of each well and representative images were examined.

\subsubsection{Chondrogenic differentiation potential}

High-density cellular aggregates were generated using pellet culture in accordance with a protocol published previously [11]. To form the pellets, 250,000 cells were seeded in 96 round-bottom well plates and then pellet formation was induced by centrifugation. Pellets were cultured at $37^{\circ} \mathrm{C}, 95 \%$ humidity, and $5 \% \mathrm{CO}_{2}$ until cells coalesced. After 1 day, pellets were either maintained in growth media or chondrogenic media supplemented with $0.5 \mu \mathrm{g} / \mu \mathrm{L}$ of SF from samples of either the low, medium or high protein group, or $10 \%$ FBS or 5\% PL as a control. Chondrogenic media consisted of advanced MEM containing $40 \mathrm{mg} / \mathrm{ml}$ of L-proline (Sigma-Aldrich, Saint-Louis, MO), $50 \mathrm{mg} / \mathrm{ml}$ Insulin Transferrin Selenium-premix (Gibco/Thermo Fisher Scientific), $50 \mathrm{mg} / \mathrm{ml}$ of ascorbic acid (Sigma-Aldrich), $10 \mathrm{ng} / \mathrm{ml}$ of TGF- $\beta 1$ and $0.1 \mu \mathrm{M}$ dexamethasone (Sigma-Aldrich). Pellets were maintained for up to 21 days in hypoxic $(2 \%$ oxygen) conditions using an integrated system with a cell culture hood and an 
incubator accessible through a gas-lock (I-Glove, BioSpherix, New York, NY). Cell culture medium was replaced every three days.

\subsubsection{Gene expression analysis}

For pellet culture, at least 4 pellets were pooled from each condition to obtain sufficient RNA. Pooled pellets from each time point were lysed using an 18-gauge needle and Qiazol lysis buffer (Qiagen, Hilden, Germany) to yield an RNA sample for one biological replicate. Three different biological replicates for each experimental condition were used concurrently for RNA isolation. Total RNA was isolated using the miRNeasy Micro Kit (Qiagen, Hilden, Germany) following the instructions of the manufacturer. RNA concentrations and purity levels were measured using a NanoDrop (Thermo Fisher Scientific). For baseline gene expression values, lysates were obtained prior to plating cells in pellet culture on day 0 (D0: plating day) and 7 days after culture in high-density pellets. $1 \mu \mathrm{g}$ of isolated RNA was used as a template for reverse transcription using M-MLV Reverse Transcriptase (Promega, Madison, USA). Gene expression was quantified using quantitative real-time reverse transcriptase polymerase chain reaction (qRT-PCR) with 10 ng cDNA per $10 \mu \mathrm{L}$ with QuantiTect SYBR Green PCR Kit (Qiagen) and the CFX384 Real-Time System (BioRad, Hercules, CA). Relative transcript abundance of genes of interest was normalized to AKT1 expression using the $2^{-\Delta \Delta C t}$ method. Representative chondrogenic genes (e.g COL2A1, ACAN and SOX9) and proliferative marker MKI67 were used.

\subsubsection{Histological Analysis}

Cell pellets were collected after 21 days in culture and fixed overnight in 10\% neutral buffered formalin. Samples were then washed and dehydrated in graded series of ethanol (70\% to $100 \%)$ and processed with xylene (50\% to $100 \%)$ prior to paraffin embedding. Paraffin blocks were cut into consecutive sections of $5 \mu \mathrm{m}$ thickness using a microtome and placed onto charged microscope glass slides for histological staining. Following deparaffinization, sections were stained for 
glycosaminoglycan content using $0.5 \%$ (wt/vol) Alcian Blue 8GX dye (SigmaAldrich) and counterstained with Nuclear Fast Red (Sigma-Aldrich). Collagen type II immunohistochemistry (IHC) was also performed. After removal of paraffin and rehydration, sections were washed with distilled water. Antigen retrieval was performed using $1 \mathrm{mg} / \mathrm{mL}$ pronase for 20 minutes at $37^{\circ} \mathrm{C}$ followed by $1 \mathrm{mg} / \mathrm{mL}$ collagenase for 60 minutes at $37^{\circ} \mathrm{C}$, both in a humidified chamber. Briefly, protein block was applied using $1 \mathrm{x}$ TBS $0.1 \%$ Tween-20 buffer after which slides were incubated overnight with $1 \mu \mathrm{g} / \mathrm{mL}$ of anti-Collagen Type II mouse monoclonal Antibody (Millipore, Darmstadt, Germany) or anti-IgG mouse monoclonal Antibody (Biosciences, Osage, IA) as a control at $4^{\circ} \mathrm{C}$ in a humidified chamber. Slides were sequentially incubated with biotinylated anti-goat IgG secondary antibody and were then treated with 3,3'-diaminobenzidine (DAB) enhanced liquid substrate system for IHC (Sigma-Aldrich) for 10 minutes until color development. Stained sections were analyzed using a ZEISS Axio inverted light microscope.

\subsubsection{Cytokine analysis.}

Human interleukin 10 (IL-10), interleukin 6 (IL-6), interleukin 1- $\beta$ (IL-1ß) and tumor necrosis factor alpha $(\mathrm{TNF} \alpha)$ concentrations were measured with a human enzyme-linked immunosorbent assay (ELISA) kits (R\&D, Minneapolis, MN, USA), according to the manufacturer's instructions. Synovial fluid samples were diluted according to the manufacturers' instructions. The electrochemiluminescence was measured using a Nanospectometer. Given the nonparametric nature of the data, the cytokines concentrations were Log2 transformed.

\subsubsection{Statistical analysis.}

Quantitative data for gene expression profiles were presented as mean \pm standard deviation from 3 independent donors for each experimental condition and time point. Statistical analysis was performed using JMP® Pro v10.0.0 (SAS Institute), and $p$ values were determined with the non-parametric comparison 
for all pairs using Steel-Dwass Method. Significance is noted in the figures when applicable as * for $p$-value $<0.05$ and ${ }^{* *}$ for $p$-value $<0.01$.

\subsection{RESULTS}

\subsubsection{Patient cohort}

The current analysis included synovial fluid samples from knees from 54 patients undergoing knee replacement surgery or knee drainage to treat flare-ups of joint disease. Table 6.1 summarized all analysis conducted on the 54 collected samples. In Table 6.2 the samples from this cohort were categorized according to their OA grade, 2 patients (3.70\%) had no visible radiological features of OA (G0), 14 patients (25.92\%) had mild OA (G1/2), 18 patients (33.33\%) had moderate OA (G3) and 20 patients (37.04\%) had severe OA (G4). Age and gender were described for each group. Thirty (56.00\%) women and twenty-four (44.00\%) men were included in the study. The mean \pm SD age of patients was $62.3 \pm 13.6$ years (range, 21-87 years). Mean age was observed to increase with KL score. In general, no predominant knee was found in patients, 25 samples were collected from the right knee whereas 25 were collected from the left knee.

\subsubsection{Sample characterization}

Physical examination was performed on the samples. The mean volume was 22 $\mathrm{mL}$ with a range from 4 to $115 \mathrm{~mL}$. Color and clarity of each sample was observed and recorded. Samples were categorized in 4 groups; transparent samples were categorized as normal, light yellow samples as non-inflammatory, dark yellow and cloudy samples as inflammatory whereas red/brown samples as hemorrhagic. None of the samples were colorless and clear or classified as normal SF, 31 samples were light yellow and clear and classified as noninflammatory OA, 20 samples were dark yellow and cloudy and classified as inflammatory $\mathrm{OA}$ and 2 samples were red/brown and classified as septic or hemorrhagic samples (Figure 6.1). 
Table 6.1 Summarized samples characteristics

\begin{tabular}{|c|c|c|c|c|c|c|c|c|c|c|}
\hline \multicolumn{4}{|c|}{ Donor characteristics } & \multicolumn{3}{|c|}{ Clinical Parameters } & \multicolumn{4}{|c|}{ Biochemical Parameters } \\
\hline $\begin{array}{l}\text { Sample } \\
\text { ID }\end{array}$ & Gender & $\begin{array}{c}\text { Age } \\
\text { (years) }\end{array}$ & $\begin{array}{l}\text { Anatomical } \\
\text { Side }\end{array}$ & $\begin{array}{l}\text { Fluid Vol } \\
\text { (mL) }\end{array}$ & Appearance & $\begin{array}{c}O A \\
\text { grade }\end{array}$ & $\begin{array}{l}\text { Protein } \\
\text { content } \\
\text { (ug/uL) }\end{array}$ & $\begin{array}{l}\text { Chondrocytes } \\
\text { Viability (abs) }\end{array}$ & $\begin{array}{c}\text { AMSCs } \\
\text { Viability } \\
\text { (abs) }\end{array}$ & $\begin{array}{c}\text { Average } \\
\text { Viability (abs) }\end{array}$ \\
\hline 1 & M & 76 & $\mathrm{R}$ & 8 & Non irflammatory & G4 & 26.91 & 1.14 & 1.03 & 1.08 \\
\hline 2 & $M$ & 76 & $\mathrm{~L}$ & 5 & Milkanmetory & G4 & 30.57 & 0.86 & 0.82 & 0.84 \\
\hline 3 & M & $\pi$ & L & 20 & Non-hifaminatory & 63 & 41.52 & 122 & 1.09 & 1.15 \\
\hline 5 & $\mathrm{~F}$ & 47 & $\mathrm{~L}$ & 5 & Milamumatory & G2 & 70.86 & 1.01 & 1.04 & 1.02 \\
\hline 6 & $\mathbf{M}$ & 40 & $\mathrm{~L}$ & 16 & bilkammatory & 62 & 2220 & 1.11 & 0.95 & 1.03 \\
\hline 7 & $\mathrm{~F}$ & 45 & $\mathbf{R}$ & 12 & Iflammatory & Go) & 75.24 & 107 & 0.99 & 1.03 \\
\hline 8 & M & 65 & $\mathbf{L}$ & 16 & 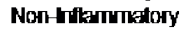 & G3-4 & 20.58 & 1.04 & 0.88 & 0.96 \\
\hline 9 & $M$ & 55 & L & 27 & 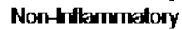 & G2 & 27.74 & 0.93 & 0.83 & 0.88 \\
\hline 10 & M & 52 & $\mathrm{~L}$ & $\mathbf{8}$ & 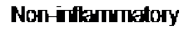 & G2-3 & 83.76 & 1.09 & 1.02 & 1.06 \\
\hline 11 & $M$ & 52 & $\mathbf{R}$ & 17 & Non irfammatory & G3 & 46.86 & 1.01 & 0.96 & 0.98 \\
\hline 12 & $\mathrm{~F}$ & 57 & $\mathbf{R}$ & 4 & Non-inflarinetory & G3 & 19.48 & 1.18 & 1.03 & 1.10 \\
\hline 13 & M & 61 & $\mathbf{L}$ & 24 & Irikamatory & G4 & 2276 & 1.27 & 0.94 & 1.10 \\
\hline 14 & $\mathrm{~F}$ & 65 & L & 12 & Non-inflariritistory & 64 & 55.16 & 1.19 & 0.84 & 1.02 \\
\hline 15 & $\mathrm{~F}$ & 78 & $\mathrm{R}$ & 12 & Non irflammzotory & G4 & 56.81 & 1.19 & 0.81 & 100 \\
\hline 16 & F & 57 & $\mathrm{R}$ & 12 & Non-inflammatory & G3 & 83.85 & 1.08 & 0.91 & 0.99 \\
\hline 17 & $M$ & 21 & $\mathbf{R}$ & 4 & Non-iflaminatory & Go) & 37.91 & NA & NA & NA \\
\hline 18 & $\mathrm{~F}$ & 80 & L & 13 & Non Irflamintatory & G4 & 48.74 & 1.38 & 0.89 & 1.13 \\
\hline 19 & $M$ & 51 & L & 9 & Iflaminktory & G2 & 59.41 & 106 & 0.90 & 0.98 \\
\hline 20 & $M$ & 57 & L & 48 & Irflammatory & G3 & 40.50 & 1.22 & 0.99 & 1.10 \\
\hline 21 & M & 57 & $\mathrm{R}$ & 50 & Irilaminatory & G3 & 46.83 & 1.05 & 0.94 & 0.99 \\
\hline 22 & $\mathrm{~F}$ & 63 & $\mathbf{R}$ & 11 & Non-hiflarninatory & G2 & 71.65 & 1.11 & 0.90 & 1.01 \\
\hline 23 & $\mathrm{~F}$ & 53 & L & 5 & Non inflantititory & G3 & 30.80 & 0.6 & 0.79 & 0.88 \\
\hline 24 & $\mathrm{~F}$ & 85 & $\mathbf{R}$ & 5 & rflammeatory & G4 & NA & NA & NA & NA \\
\hline 25 & $F$ & 57 & $\mathbf{L}$ & 40 & Irilammatory & G3 & 10463 & 096 & 0.83 & 0.90 \\
\hline 26 & $\mathrm{~F}$ & 76 & $\mathbf{R}$ & 5 & Non iritammatory & G2 & 2289 & 0.87 & 0.71 & 0.79 \\
\hline 27 & $\mathrm{~F}$ & 79 & $\mathbf{L}$ & 12 & Iflamumestory & 64 & NA & NA & NA & NA \\
\hline 28 & $\mathrm{~F}$ & 64 & $\mathbf{R}$ & 110 & Non inflantitifory & G3 & 41.78 & 0.85 & 1.07 & 0.96 \\
\hline 29 & $\mathrm{~F}$ & 56 & $\mathbf{R}$ & 28 & Ifleminkatory & 64 & 2702 & NA & NA & NA \\
\hline 30 & $\mathrm{~F}$ & 74 & $\mathbf{L}$ & 14 & Non-iflarmetory & 64 & 25.01 & 1.20 & 1.03 & 1.12 \\
\hline 31 & $\mathrm{~F}$ & 71 & $\mathrm{R}$ & 5 & Non inflammatory & $\mathbf{G 3}$ & 23.78 & 1.24 & 0.93 & 1.09 \\
\hline 32 & $M$ & 64 & $\mathbf{R}$ & 7 & Non-inflannatory & G3 & 26.71 & 0.83 & 0.89 & 0.86 \\
\hline 33 & $M$ & 62 & $\mathbf{R}$ & 34 & Milkytumestoy & G2 & 3418 & 0.83 & 0.89 & 0.86 \\
\hline 34 & $M$ & 46 & $\mathrm{R}$ & 8 & Irifammatory & G2 & 26.58 & 1.02 & 0.85 & 0.93 \\
\hline 35 & $M$ & 62 & $\mathbf{R}$ & 58 & Milkamatory & 63 & 44.90 & 0.70 & 0.99 & 0.85 \\
\hline $36 \mathrm{~L}$ & M & $\pi$ & $\mathbf{L}$ & 70 & Non irflammatory & G4 & 63.68 & 1.08 & 1.01 & 1.05 \\
\hline 37 & $M$ & $\pi$ & $\mathbf{R}$ & 115 & Non-inflamiritolory & 64 & 1823 & 0.90 & 1.13 & 102 \\
\hline 38 & $\mathrm{~F}$ & 79 & L & 21 & Non inflammatory & G4 & 1853 & 0.95 & 1.04 & 0.99 \\
\hline 39 & $\mathrm{~F}$ & 79 & $\mathrm{R}$ & 51 & Non inflammatory & 64 & 2283 & 1.21 & 0.88 & 1.04 \\
\hline 40 & $\mathrm{~F}$ & 53 & $\mathbf{L}$ & 32 & Non irflammatory & G1 & 129.18 & 0.76 & 0.96 & 0.86 \\
\hline 41 & M & 67 & $\mathbf{L}$ & 5 & Milkatumetory & G4 & 17.57 & 0.72 & $0 . \pi$ & 0.75 \\
\hline 42 & $M$ & 55 & $\mathbf{R}$ & 17 & Inflaminatory & G4 & 21.91 & 0.85 & 0.86 & 0.86 \\
\hline 43 & $\mathrm{~F}$ & 54 & L & 10 & Hemorraghix & G1 & 37.96 & 1.04 & 1.13 & 1.08 \\
\hline 44 & F & 87 & $\mathbf{L}$ & 24 & Non hiflaminetory & 63 & 26.85 & 1.19 & 1.12 & 1.15 \\
\hline 45 & $\mathrm{~F}$ & 51 & $\mathbf{R}$ & 10 & Non-riftarintibtory & G2 & 35.89 & 0.88 & 1.15 & 1.01 \\
\hline 46 & $M$ & 30 & L & 8 & Non inflantitatoy & G1 & 6920 & 0.74 & 1.12 & 0.93 \\
\hline 47 & $\mathrm{~F}$ & 67 & L & 23 & Iflaminatory & G2 & 3500 & 0.67 & 1.19 & 0.93 \\
\hline 48 & $F$ & 72 & $\mathbf{L}$ & 6 & Non Irflammatory & G4 & 78.83 & 0.87 & 1.04 & 0.95 \\
\hline 49 & $\mathrm{~F}$ & 69 & $\mathrm{R}$ & 32 & Non hifarmatory & G4 & 75.79 & 1.00 & 1.00 & 1.00 \\
\hline 50 & $\mathrm{~F}$ & 61 & $\mathbf{R}$ & 11 & Iflamentory & G2-G3 & 17.44 & 0.85 & 0.99 & 0.92 \\
\hline 51 & $M$ & 81 & L & 37 & Irikatumatory & G4 & $34 \pi$ & 1.01 & 0.93 & 0.97 \\
\hline 52 & $\mathrm{~F}$ & 58 & $\mathbf{R}$ & 13 & Hernorraghix & G2 & 24.60 & 1.12 & 1.02 & 1.07 \\
\hline 53 & $M$ & 56 & NA & 25 & Non-Irflammatory & G3 & 1205 & 0.96 & 1.56 & 1.26 \\
\hline 54 & $\mathrm{~F}$ & 51 & NA & 10 & Non hilamerinatory & G3 & 10.78 & 0.93 & 1.59 & 1.26 \\
\hline
\end{tabular}

Note: Viability assays values are presented as absorbance at $490 \mathrm{~nm}$ relative to media alone.

Also, chemical examination was performed on the samples. An initial quantification of undiluted samples was performed. Protein concentration value 
quantification was checked by performing a dilution series of each sample. Protein content was determined when linearity of the measurements was observed.

Table 6.2 Patient demographics.

\begin{tabular}{|c|c|c|c|c|}
\hline Grade $\mathbf{O A}$ & $\begin{array}{c}\text { No OA } \\
\text { (G0) }\end{array}$ & $\begin{array}{c}\text { Mild OA } \\
\text { (G1-2) }\end{array}$ & $\begin{array}{c}\text { Moderate OA } \\
\text { (G3) }\end{array}$ & $\begin{array}{c}\text { Severe OA } \\
\text { (G4) }\end{array}$ \\
\hline \#Samples & 2 & 15 & 17 & 21 \\
\hline $\begin{array}{c}\text { Anatomy } \\
\text { (Right, Left) }\end{array}$ & $\begin{array}{c}\text { Knee } \\
(2 \mathrm{R}, 0 \mathrm{~L})\end{array}$ & $\begin{array}{c}\text { Knee } \\
(6 \mathrm{R}, 9 \mathrm{~L})\end{array}$ & $\begin{array}{c}\text { Knee } \\
(10 \mathrm{R}, 7 \mathrm{~L})\end{array}$ & $\begin{array}{c}\text { Knee } \\
(8 \mathrm{R}, 13 \mathrm{~L})\end{array}$ \\
\hline $\begin{array}{c}\text { Age, mean } \\
\text { (range) }\end{array}$ & $\begin{array}{c}33 \\
(21-45)\end{array}$ & $\begin{array}{c}54 \\
(30-76)\end{array}$ & $\begin{array}{c}63 \\
(52-87)\end{array}$ & $\begin{array}{c}74 \\
(56-85)\end{array}$ \\
\hline $\begin{array}{c}\text { Gender } \\
\text { (\% Female) }\end{array}$ & $60 \%$ & $57 \%$ & $54 \%$ & $61 \%$ \\
\hline
\end{tabular}

After protein content was quantified, SF samples were ranked based on real value protein concentration and categorized according to OA grade. As shown in Figure 6.1.A SF protein content was higher in patients with no or mild OA, while samples with a lower protein content were collected from patients with moderate and/or severe OA grades. This suggests that protein content might be associated with disease state with concentration decreasing along $\mathrm{OA}$ development. Comparison of fluid volume and samples clarity against OA grade was also performed. Contrarily to protein content, fluid volume of samples increased in samples during OA development Figure 6.1.B, whereas samples clarity showed clear differences between the samples. Moderate and severe SF samples presented more non-inflammatory aspect of the fluid; whereas mild OA samples appeared to be more inflammatory and hemorrhagic Figure 6.1.C. Samples were normalized to an equal protein concentration using PBS and used for further experimental analysis. 


\subsubsection{Sample stratification.}

Stratification methods were examined to determine a better categorization for further experimentation using MSC and human chondrocyte cultures. Donor characteristics and chemical analysis of the samples were compared among the OA grades. From Table 6.3, we observed that slightly more severe OA samples and females were included in the study. Age and volume aspirated increased with OA progression. However, protein concentration decreased with OA progression. The effects of disease state of the synovial fluid samples on the viability of aMSC and chondrocyte were negligible. Protein yield quantification also suggested that protein content increases in OA SF samples compared to no OA samples.

A

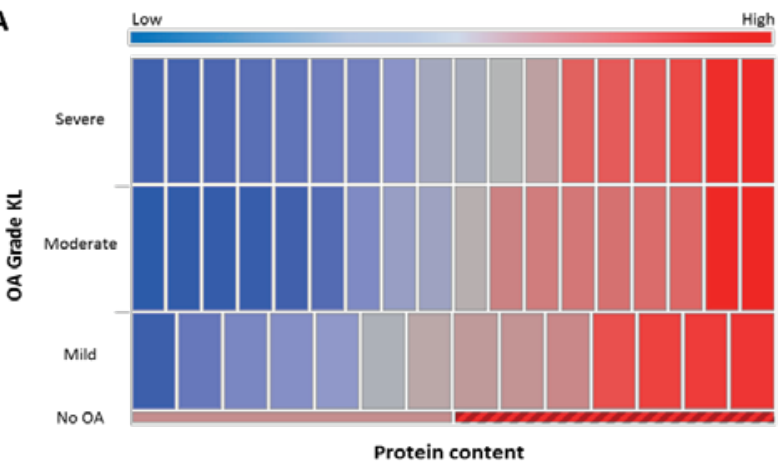

B

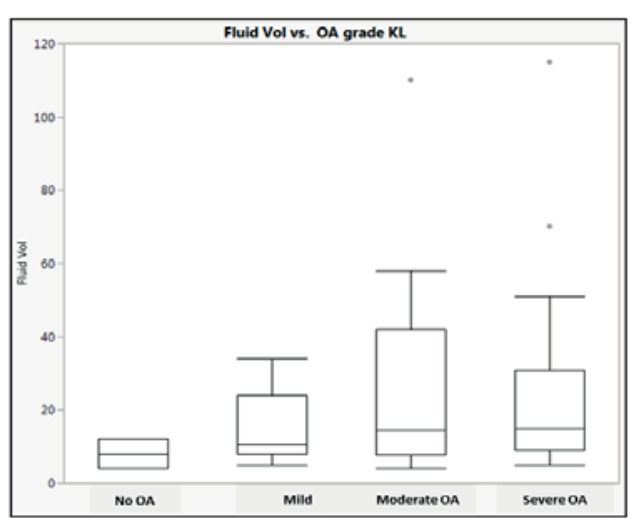

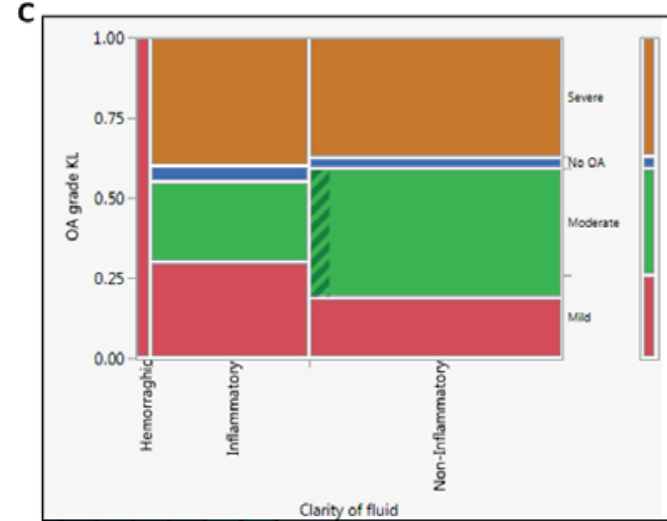

Clarity of fluid

Figure 6.1. Categorization of SF samples according to OA grade to examine protein content (A),

fluid volume of samples (B) and fluid clarity (C). Results shown in panel A and C are represented as a mosaic plot, results in panel B are represented as median and 95\% confidence intervals (CI). 
Table 6.3 Samples stratification according to OA classification.

\begin{tabular}{|ccccc|}
\hline & $\begin{array}{c}\text { No OA } \\
\text { (G0) }\end{array}$ & $\begin{array}{c}\text { Mild OA } \\
\text { (G1-2) }\end{array}$ & $\begin{array}{c}\text { Moderate OA } \\
\text { (G3) }\end{array}$ & $\begin{array}{c}\text { Severe OA } \\
\text { (G4) }\end{array}$ \\
\#Samples & 2 & 14 & 18 & 20 \\
Age (mean \pm stdev) & $33 \pm 16.97$ & $54.14 \pm 11.88$ & $61.87 \pm 9.80$ & $73.83 \pm 7.69$ \\
$\begin{array}{c}\text { Gender (\% Females) } \\
\text { Volume aspirated } \\
\text { (mean } \pm \text { stdev) }\end{array}$ & $50 \%$ & $57 \%$ & $56 \%$ & $55 \%$ \\
$\begin{array}{c}\text { Protein concentration } \\
\text { (mean } \pm \text { stdev) }\end{array}$ & $56.57 \pm 26.39$ & $47.67 \pm 29.83$ & $45.31 \pm 25.83$ & $39.14 \pm 20.83$ \\
$\begin{array}{c}\text { Protein Yield (mg) } \\
\text { Chondro MTS assay } \\
\text { (mean } \pm \text { stdev) }\end{array}$ & $1.07 \pm 0.11$ & 773 & 1160 & 948 \\
$\begin{array}{c}\text { AMSCs MTS assay } \\
\text { (mean } \pm \text { stdev) }\end{array}$ & $0.99 \pm 0.21$ & $0.94 \pm 0.15$ & $1.02 \pm 0.17$ & $1.05 \pm 0.15$ \\
\hline
\end{tabular}

SF samples were divided in to low $(<30 \mu \mathrm{g} / \mu \mathrm{L})$, medium (30 to $60 \mu \mathrm{g} / \mu \mathrm{L}$ ) and high $(>60 \mu \mathrm{g} / \mu \mathrm{L})$ protein groups for future cell culture experiments. Protein content was presented in a Log2 scale (Figure 6.2). Fetal bovine serum (FBS) and human platelet lysate (PL) that were used for experimental controls had a protein content of $35 \mu \mathrm{g} / \mu \mathrm{L}$ and $17.93 \mu \mathrm{g} / \mu \mathrm{L}$, respectively. $90 \%$ of the samples in the whole set had a protein content higher than PL. Samples with high protein content were around $46 \%$ of the whole set (24 samples), medium protein group were 44\% (23 samples) and 9.60\% (5 samples) in the low protein group. The synovial fluid samples that presented better viability in aMSCs had more protein content and its protein values were divided across the three protein groups (6 to low, 5 to medium and 7 to high protein content group), whereas chondrocytes were more viable in samples with lower protein content (11 samples in low group, 10 in medium group and 7 in high protein content group).

Moreover, synovial fluid protein quantification revealed variability in protein content among the samples per group of $29.20 \pm 11.90,57.30 \pm 9.50$ and $91.10 \pm$ $6.30 \mu \mathrm{g} / \mu \mathrm{L}$, for the low, medium and high protein group respectively. Although this stratification gives a good amount of information about OA role in SF characterization, the cell's viability difference was not clear. For this, a 
stratification of the samples according to MTS viability assay was suggested. MTS results from each cell type were examined in Table 6.4.

In Table 6.4.A and Figure 6.3.A we show that human chondrocytes preferred SF samples with a lower protein yield and advanced OA grades. Interestingly, 50\% of the samples improved the viability of the cells compared to media alone. No statistical difference in age or OA grade was found. As observed previously, chondrocytes were more active when protein yield was low. Also, Table 6.4.B and Figure 6.3.B show aMSCs stratification according to their MTS viability assays. Only $35 \%$ of the samples provide better viability than media alone. As validated before, aMSCS preferred samples with higher protein yield. Age at aspiration did not have a role in aMSCs or chondrocytes viability.

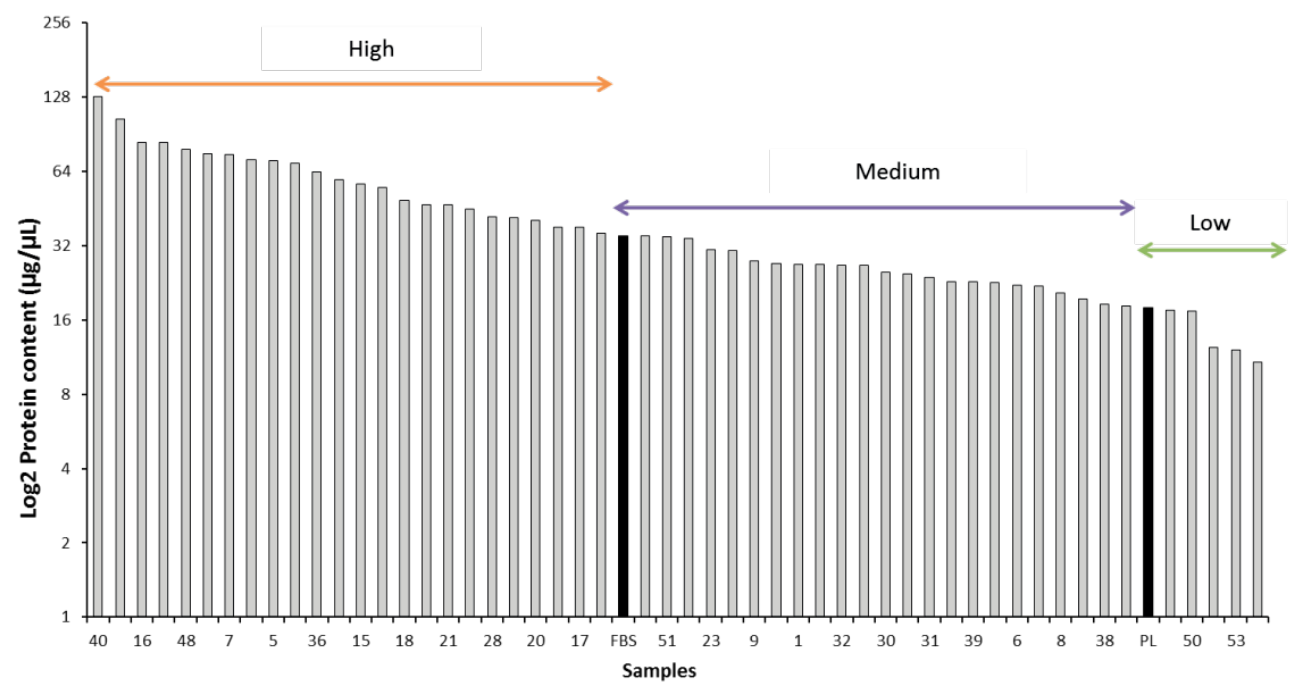

Figure 6.2. Protein content division in three different groups: High, medium and low. All SF samples are represented in gray bars whereas control serums, FBS and PL, are represented in black bars. 
Table 6.4 Samples stratification according to MTs viability assays.

\begin{tabular}{|c|c|c|c|}
\hline Viability & $\leq 0.7$ & $\underline{0.7-1.0}$ & 1.0-1.4 \\
\hline \#Samples & 2 & 20 & 27 \\
\hline $\begin{array}{c}\text { Age } \\
\text { (mean } \pm \text { stdev) }\end{array}$ & $64.5 \pm 3.54$ & $61.4 \pm 11.85$ & $63.41 \pm 13.13$ \\
\hline Gender ( $\%$ Females) & $50 \%$ & $55 \%$ & $52 \%$ \\
\hline Grade $O A$ & Mild-Moderate & Moderate-Severe & Moderate-Severe \\
\hline Protein Yield (mg) & 1705 & 1134 & 837 \\
\hline $\begin{array}{l}\text { Protein content } \\
\text { (mean } \pm \text { stdev) }\end{array}$ & $44.76 \pm 27.48$ & $42.24 \pm 22.24$ & $11.41 \pm 0.90$ \\
\hline Viability & $\underline{0.7-1.0}$ & $1.0-1.4$ & $\geq 1.4$ \\
\hline \#Samples & 30 & 17 & 2 \\
\hline $\begin{array}{c}\text { Age } \\
\text { (mean } \pm \text { stdev) }\end{array}$ & $62.1 \pm 10.90$ & $64.64 \pm 14.82$ & $53.5 \pm 3.54$ \\
\hline Gender & $47 \%$ & $65 \%$ & $50 \%$ \\
\hline Grade OA & Moderate-Severe & Mild-Moderate & Mild \\
\hline Protein Yield (mg) & 1027 & 1034 & 1560 \\
\hline $\begin{array}{l}\text { Protein content } \\
\text { (mean } \pm \text { stdev) }\end{array}$ & $35 \pm 0$ & $41.41 \pm 32.02$ & $43.67 \pm 20.83$ \\
\hline
\end{tabular}

Note: Values are presented as fold change of viability of SF samples over media alone. $<0.7$ were inhibitory samples, 0.7-1.0 were neutral samples and $>1.0$ were stimulatory samples for each cell type.

However, OA grade seems to affect viability of the cells. aMSC were slightly more viable in mild OA samples whereas chondrocytes grew better in moderate and severe OA samples. This suggested that aMSC are more affected by the content of synovial fluid and the OA grade. Further comparison of the similarities and differences in the response of aMSCs and chondrocytes to similar SF samples was performed. 27 samples elicited a different response in aMSCs and chondrocytes whereas 28 samples resulted in a similar response according to MTS cell viability as a read out (samples with MTS viability of more than 1 in both cells, see Table 6.1). Also, we found that $16 \%$ of all SF samples behaved in a similar manner in both cell types. In this case, samples that provided viability better than media alone for both cells were categorized in the medium and high protein content groups. 


\subsubsection{Viability of mesenchymal stem cells and chondrocytes in synovial fluid}

To investigate if synovial fluid maintains metabolic activity of mesenchymal stem cells and human chondrocytes viability assays and growth curves were performed. These assays were used to determine the optimal synovial fluid concentration for culturing each cell type in vitro. For cell culture experiments, all synovial fluid samples were normalized to a protein concentration of $10 \mu \mathrm{g} / \mu \mathrm{L}$ in PBS. Metabolic activity of human mesenchymal stem cells culture in SF revealed differences between the low, medium and high protein content groups. Cells were grown in a volume per volume (v per v) or two-fold dilutions series of SF samples (Figure 6.4.A). Volume per volume was diluted starting from $1 / 5$ dilution until 1/160 in media. Dilutions of $1 / 2$ were added due to the significance in a clinical setting, since stem cells injections for knee therapy are diluted in a $1 / 2$ concentration. Two-fold dilutions were performed starting from the normalized samples with $10 \mu \mathrm{g} / \mu \mathrm{L}$ concentration and diluted up to $0.3 \mu \mathrm{g} / \mu \mathrm{L}$ in media.

A

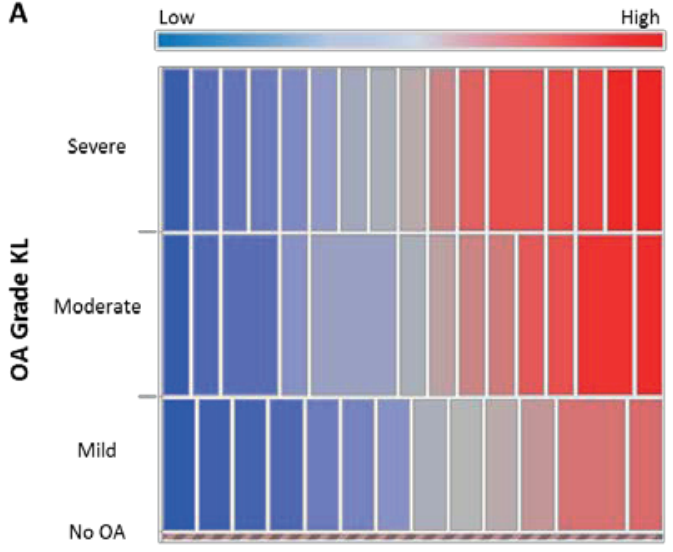

Viability Chondrocytes

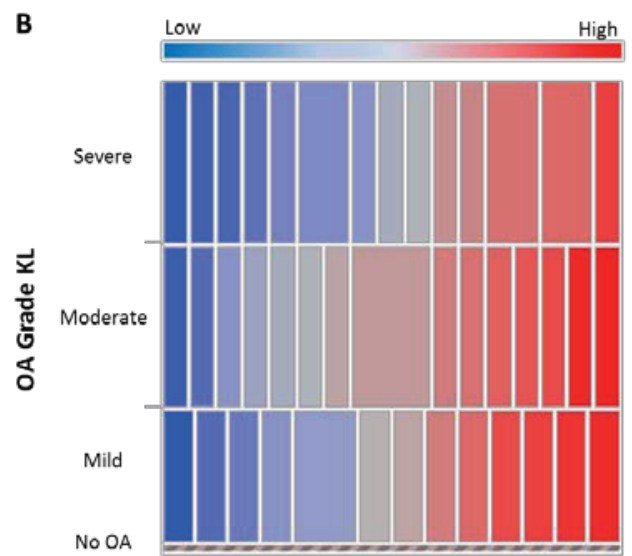

Viability AMSCs

Figure 6.3 Mosaic plot of MTS viability assays of (A) aMSCs and (B) chondrocytes compared to OA grade. 

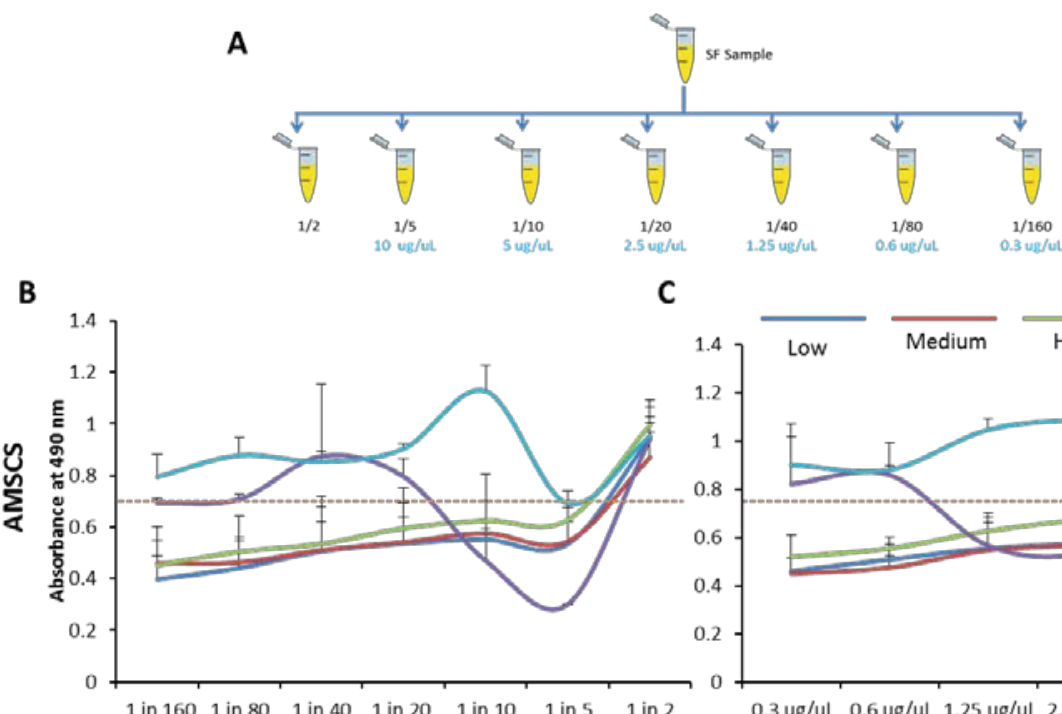

C
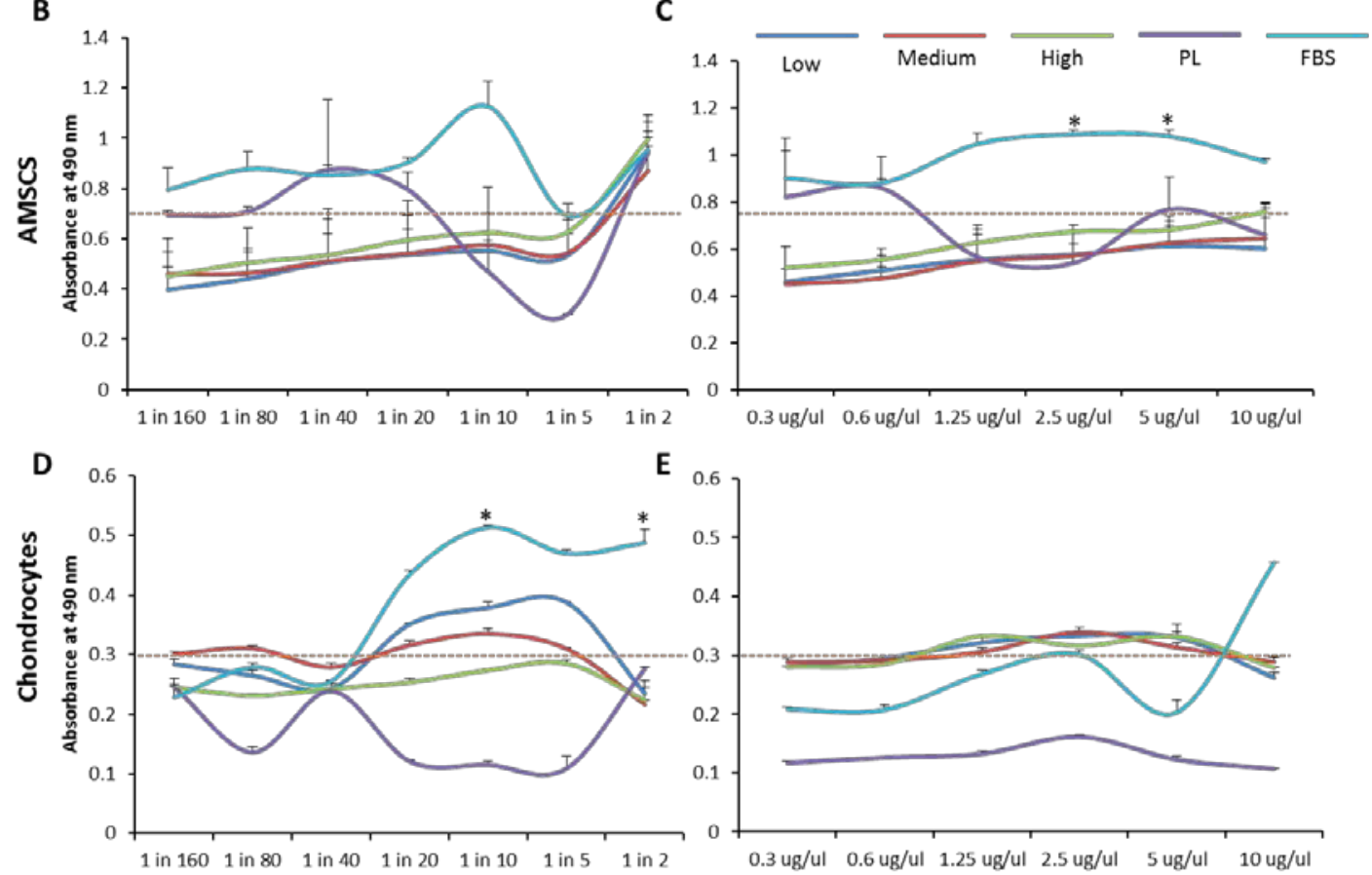

Figure 6.4. Cell viability assays in SF serum of three different groups compared to FBS and PL. (A) Volume per volume (black) dilutions and normalized two-fold dilutions (blue). Viability assays on human AMSCS (B and C) and human primary chondrocytes (D and E). Data represented as mean \pm STDEV. Three biological replicates $(n=3)$ and three technical replicates were used.

All dilutions were performed using normalized samples to the same concentration to avoid variability due to protein content. Relative metabolic activity to media alone in proliferative aMSC revealed that both two-fold dilutions and $\mathrm{v}$ per $\mathrm{v}$ had a similar metabolic activity. FBS was the serum that induced more metabolic activity whereas high protein group had an enhanced metabolic activity across all dilutions when compared to medium and low content group (Figure 6.4.B and C). Despite the normalization of the samples to 
the same protein content, samples from each group interacted in a different manner with aMSC, which suggest the content (e.g. proteins, cytokines, etc) of each group can affect the behavior of the cells. In lower dilutions SF groups were more similar, but differed significantly from FBS and PL. However, in higher dilutions (e.g. $1 / 2$ and $1 / 5$ ) the difference between PL, FBS and SF samples is reduced. In some dilutions, 1 in 10, 1 in 5 and 1 in 2 and $1.25 \mu \mathrm{g} / \mu \mathrm{L}$ and 2.5 $\mathrm{ug} / \mathrm{uL}$, an increase in metabolic activity of the cells in SF compared to PL was observed. By comparing the clinical relevant dilution (e.g. 1/2) we observed that the majority of samples had a metabolic activity higher than 0.7. Yet, a slight increase in high protein SF compared to other SF groups, FBS and PL was detected.

Metabolic activity of human chondrocyte culture in SF revealed differences between the low, medium and high protein content groups when cultured in volume per volume dilutions (Figure 6.4.D). Chondrocytes grew in synovial fluid derived from patients with low and medium protein concentrations showed an enhanced metabolic activity compared to those grown in synovial fluid from patients with high protein concentrations. Along all dilutions, SF with low protein content and FBS were the ones with better metabolic activity whereas PL was the serum with lower metabolic activity. Dilutions between $1 / 20$ and $1 / 5$ seemed better to culture chondrocytes. In these dilutions medium and low protein SF samples were better than PL and slightly similar to FBS. By comparing the $1 / 2$ dilution that is commonly used for stem cell delivery, we observed all SF samples decreased compared to PL and FBS. This can be due to a serum shock when protein volume is too high. Contrarily, in lower dilutions (e.g. 1/40, 1/80 and $1 / 160$ ) metabolic activity increased in synovial fluid samples compared to FBS and PL.

A comparison between the two-fold diluted normalized samples showed no significant variation between dilutions in low, medium and high protein content 
groups (Figure 6.4.E). All SF samples showed an enhanced metabolic activity compared to FBS and PL, except in the stock solution $(10 \mu \mathrm{g} / \mu \mathrm{L})$ were FBS was significantly enhanced. Overall, in culture using non-normalized samples, FBS demonstrated efficient cell proliferation and cell viability compared to other serums. In aMSC, both FBS and PL showed a good viability compared to SF samples. For human chondrocyte, PL serum significantly decreased the viability of the cells whereas SF samples did almost as good as FBS. In contrast to aMSC, chondrocyte prefer to be seeded in low protein content SF. In culture with normalized serums, both cells exhibited different behavior. aMSC preferred FBS, PL and high protein content SF samples, whereas chondrocyte showed higher viability in all SF samples compared to FBS and PL. Synovial fluid from patients with more severe OA typically had a higher protein concentration due to increased inflammatory proteins, and may therefore be less suitable for promoting chondrocyte growth and viability. For cell culture purposes, either normalized dilutions or volume per volume dilutions can be used because a similar behavior was observed. The ideal SF concentrations identified in these experiments were: for 1 ) volume per volume dilutions: $1 / 20,1 / 10,1 / 5$ and $1 / 2$ and 2) Two-fold normalized dilutions $1.25,284.50$ or $5 \mu \mathrm{g} / \mu \mathrm{L}$. Lastly, both cell types preferred more serum content over diluted serum in their growth media.

\subsubsection{Glucose importance for human chondrocytes cultured in synovial fluid.}

Since the normalization of SF samples was performed using PBS, we examined if this addition could affect the metabolic activity of chondrocytes. For this, chondrocytes were grown in different dilutions of the $10 \mu \mathrm{g} / \mu \mathrm{L}$ normalized samples. Two-fold dilutions were prepared in media or PBS and all data is represented as relative mean to PBS or media alone.

As observed in Figure 6.5.A, most of the SF samples diluted in media showed higher proliferation than samples diluted in PBS. However, in the $10 \mu \mathrm{g} / \mu \mathrm{L}$ 
dilution both dilutions are similar. Also, a slight increase of viability was observed in cells cultured in low protein containing SF. In confluent cells, the difference between cells cultured in diluted SF samples in media or PBS was more notorious. Chondrocytes were more sensitive when confluent to dilution in PBS (Figure 6.5.B). Metabolic activity of 100\% samples was higher than PBS alone, showing no cell viability at all. Thus, the significant down-regulation of chondrocytes viability in samples diluted in PBS suggested that although SF can be used as a tissue culture agent for chondrocytes it requires supplements present in media to maintain the cells proliferation and survival. Presence of glycosaminoglycans in SF does not provide enough nutrients to the cells as opposed to the high glucose content of cell culture media.

A

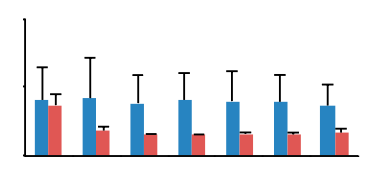

B

$\varepsilon$

Dilution in media Dilution in PBS

Figure 6.5. Cell viability assays of human primary chondrocytes cultured in SF serum of low, medium and high protein content. Proliferative (A) and confluent human primary chondrocytes

(B) were compared. SF samples diluted in media are represented in blue whereas SF samples diluted in PBS are represented in red. Data represented as relative absorbance to media or PBS alone \pm STDEV. Three biological replicates $(n=3)$ and three technical replicates were used.

\subsubsection{Growth curve assays.}

From the $1 / 2$ dilution used in the metabolic activity assays, the 4 most stimulatory and 4 most inhibitory samples were chosen for each cell type. The most 
stimulatory samples in aMSC showed higher protein yield and mild OA classification. Stimulatory samples in aMSC were SF45, SF47, SF28, SF44 and SF3 whereas inhibitory samples were SF41, SF26, SF34, SF15 and SF23. At day 0 (plating day) around 50,000 cells were seeded per well. At day 1 there is an indication that cells have a stress response and started to die, after this, cells started proliferating over time (Figure 6.6.A). This initial shock due to serum change was stronger in some samples than others. PL and FBS were the serums with better proliferation capacity whereas some SF samples had better proliferation potential than others. Although the majority of the samples showed better proliferation over time, some samples did not recover enough after 7 days in culture. They were considered not suited for cell culture. SF45 and SF47 were on the top 10 of SFs stimulating proliferation in the aMSC MTS assay. In contrast, SF23 and SF44 had the lowest proliferation capacity in aMSCS. On average stimulatory samples have higher cell numbers along the time course compared to inhibitory samples. The morphology of the cells and cell density was observed by live/dead staining at days 1 and 7 (Figure 6.6.B). FBS, PL and stimulatory SFs show a high proliferation capacity at day 7 when compared to day 1 . However, inhibitory SFs show slow proliferation and low cell number at day 7. Although stimulatory SFs increased the proliferation of aMSC, FBS and PL seemed to perform better for these cells. Also, the cells in SF look more fibroblastic than in FBS or PL. Similarly, the most stimulatory samples in chondrocytes showed lower protein yield and were linked to higher OA grades. From day 1 all SF samples, FBS and PL showed an increase in proliferation of chondrocytes along the time course (Figure 6.6.C). Some samples had better proliferation potential than others. 

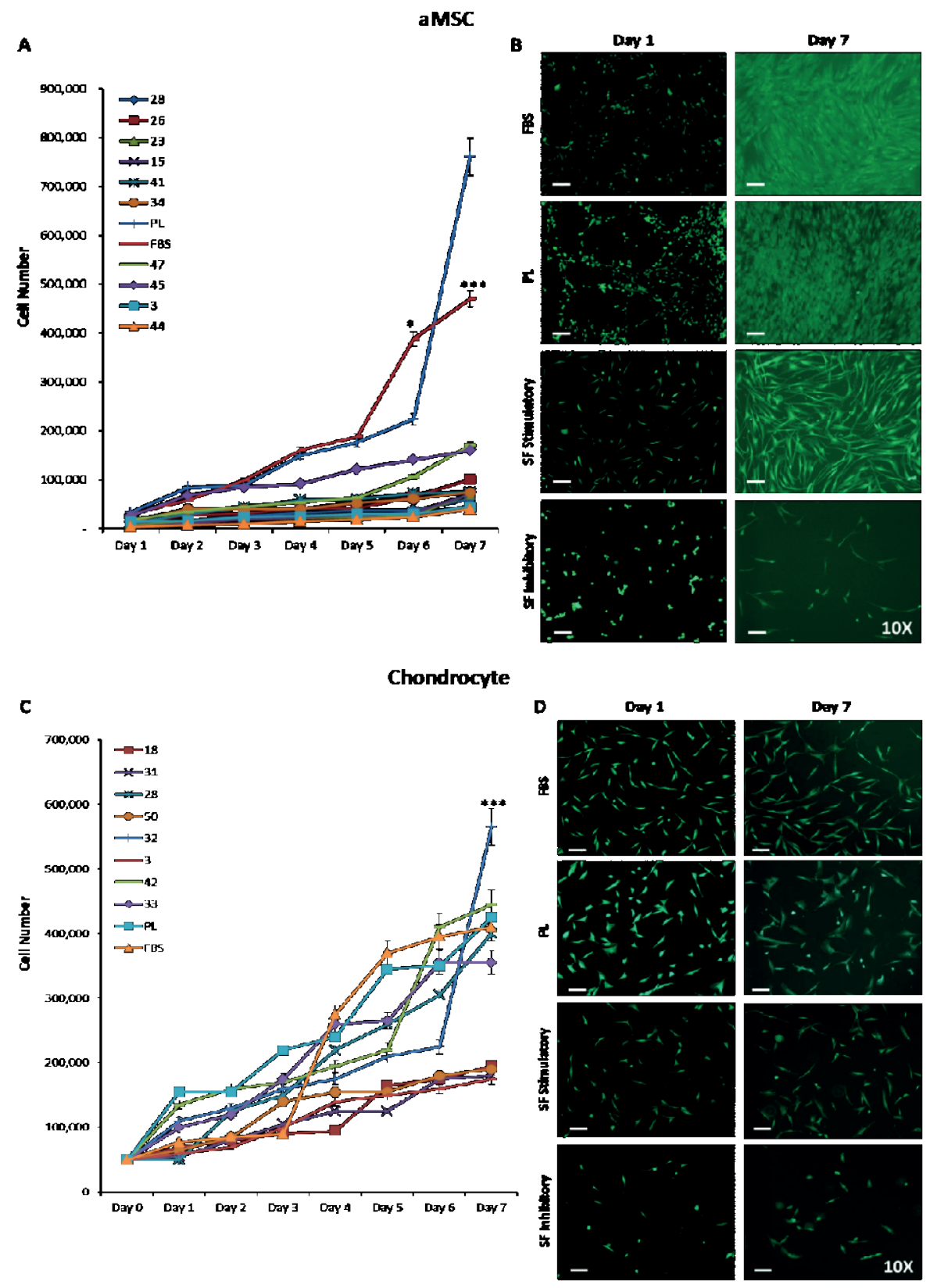

Figure 6.6. Growth curve assays in human aMSCs (A and B) and human primary chondrocytes (C and D). Data represented as mean \pm STDEV. Three biological replicates $(n=3)$ and two technical replicates were used. (Scale bar $=100 \mu \mathrm{m})$. 
Although FBS and PL showed good proliferation, three SF samples had better proliferative capacity (e.g. SF32, SF28 and SF42), and had better or similar proliferation capacity than FBS over the 7 days in culture, reaching almost a 10fold increase in cell number. In contrast, SF3, SF31, SF50 and SF18 were the SF samples with less proliferation capacity. On average, stimulatory samples had higher cell numbers along the time course compared to inhibitory samples. Only one stimulatory sample did not perform as expected (e.g. SF15). Morphology of the cells in the 4 serums did not show any significant difference (Figure 6.6.D). Still, the inhibitory SF compared to other serums significantly decreased cell density. Moreover, PL and stimulatory SF showed a similar trend in cell density at day 7.

\subsubsection{Chondrogenic differentiation.}

\subsubsection{Synovial fluid seems to maintain chondrocytic potential of primary chondrocytes in culture.}

To determine how synovial fluid affects differentiation of chondrocytes, an in vitro differentiation assay was performed using passage 4 human primary chondrocytes that were allowed to differentiate up to 21 days in three different synovial fluid samples. Samples from each group were used (e.g. low, medium and high protein content). Different serums were added in growth media or in growth media with chondrogenic cocktail. The different serums used were $10 \%$ FBS, 5\% SF and 5\% PL. Chondrocytes were seeded in high-density pellets and cultured under hypoxic conditions $(2.1 \%$ oxygen). Gene expression was examined after one week in culture. An overall comparison between SF and FBS showed some similarities between both serums. Growth media cultures containing SF and FBS up-regulated cartilage markers DCN, SOX6, SOX9 (Figure 6.7.A) and COMP (data not shown). However, COL2A1, COL1A1 and ACAN were differentially regulated. These three chondrocytic markers were higher in FBS than in SF. Moreover, a comparison between both serums with chondrogenic 
differentiation shows that SF with chondrogenic cocktail increased the expression of all chondrogenic markers compared to FBS. Proliferation marker HIST2H4 showed that SF promotes proliferation of chondrocytes when cultured in a high-density pellet under hypoxia conditions.

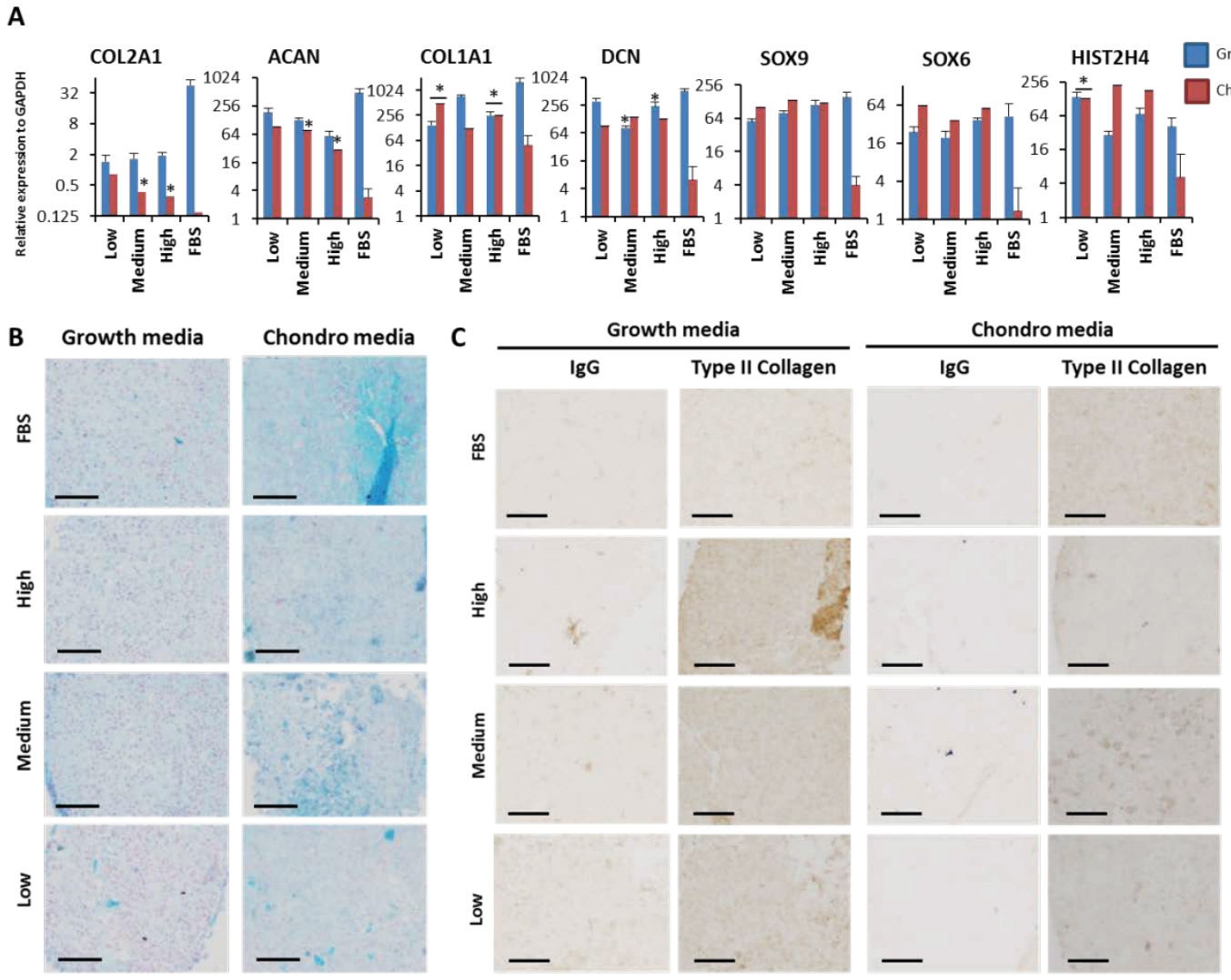

Figure 6.7. Chondrocyte culture with FBS or SF from each protein content group. (A) Gene expression analysis of cartilage specific markers and proliferation marker HIST2H4. Data represented as mean \pm STDEV. Statistical differences were noted using * if $p$-value $<0.05$ or ** if $p$ value $<0.01$. Three biological replicates $(\mathrm{n}=3)$ and three technical replicates were used. (B) Alcian blue staining of high-density pellets, (C) Collagen type II immunohistochemistry of the cultures.

(Scale bar $=100 \mu \mathrm{m})$.

Gene expression analysis also suggested that chondrocytes were proliferating after 21 days. Histological analysis of cell cultures using alcian blue staining 
revealed no differences between the SF samples and FBS when cells were grown in growth media (Figure 6.7.B). However, under chondrogenic induction, FBS and high protein content samples enhanced proteoglycan deposition compared to medium and low protein content samples which were faintly stained by alcian blue. Furthermore, analysis of collagen type II specific deposition revealed that all SF samples produced COL2A1 without the addition of chondrogenic media (Figure 6.7.C). Interestingly when chondrogenic cocktail was added to the SF samples neither of them stained slightly positive for collagen type II. However, chondrogenic cocktail did increase collagen type II production in cells plated with FBS.

\subsubsection{Synovial fluid preserves proteoglycan deposition of chondrocytes in culture and promotes chondrogenic differentiation of aMSCs in the presence of chondrogenic cocktail.}

To determine how synovial fluid affects differentiation of chondrocytes and aMSC, an in vitro chondrogenic differentiation experiment was performed. For each cell type, the 10 most stimulatory and 10 most inhibitory SF samples in terms of viability were used. From Table 6.4.A samples with viability above 1.4 for chondrocytes MTS assay were used to culture chondrocytes. From Table 6.4. $B$ samples with viability above 1.4 for aMSC MTS assay were used for aMSC culture. $10 \%$ FBS, $5 \% \mathrm{PL}$ and $5 \% \mathrm{SF}$ were used with growth or chondrogenic media. Cells were cultured in high-density pellet and incubated under hypoxia (2.1\% oxygen) conditions.

In Figure 6.8.A, we show that the potential to sustain cell viability does not correlate with chondrogenic potential in presence of SF samples. SF does not enhance expression of chondrogenic genes in chondrocytes when compared to FBS or PL. Statistical differences were found between stimulatory and inhibitory SF samples and FBS. ACAN and SOX9 increased in all cultures when compared to day 0 , whereas none of the serums was capable of preserving the COL2A1 
expression during culture when compared to day 0. Histological analysis of proteoglycan deposition using alcian blue staining was performed at day 21 .

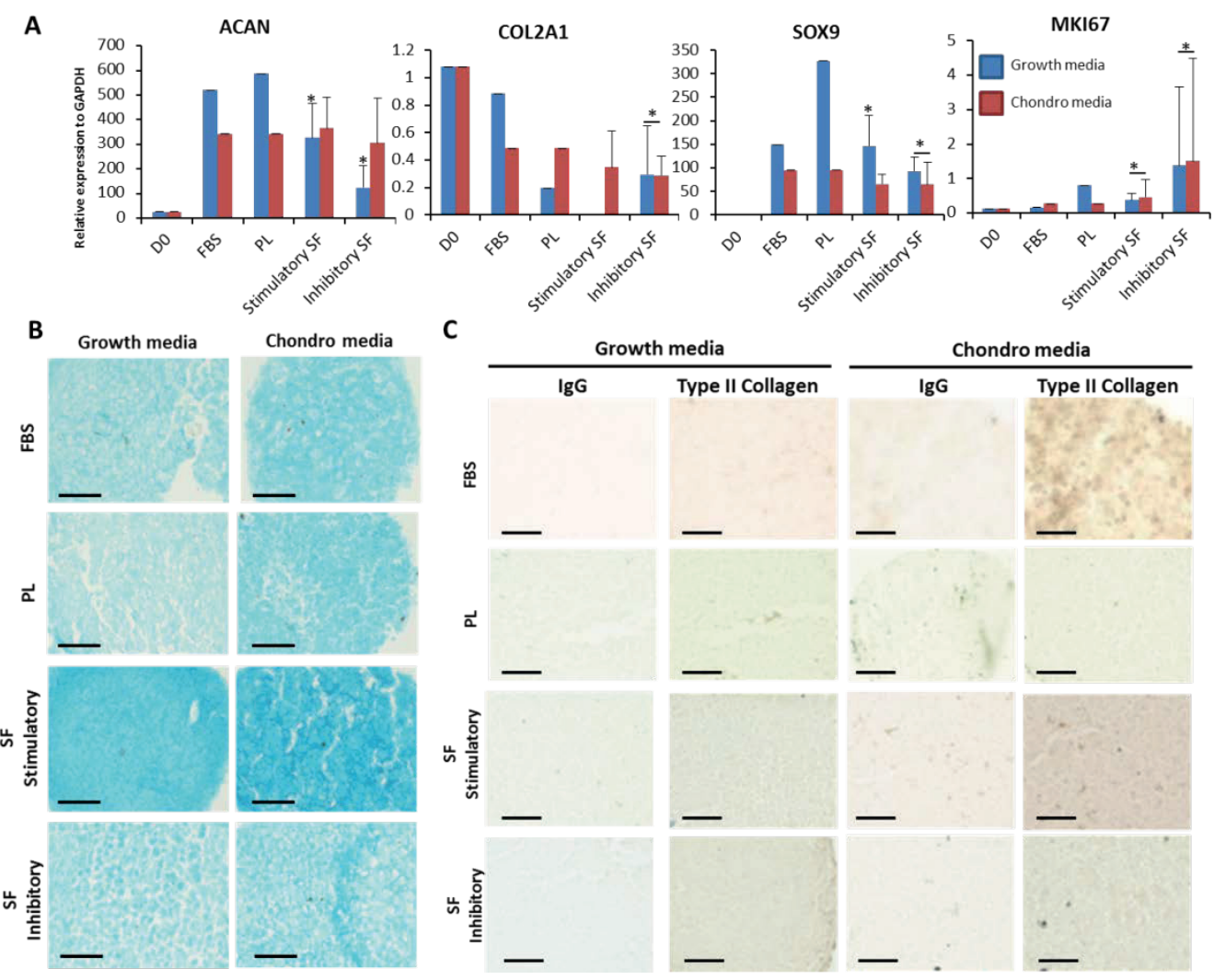

Figure 6.8. Chondrocyte cultured in FBS, PL or SF. Stimulatory or Inhibitory SF samples were used. (A) Gene expression analysis of cartilage specific markers and proliferation marker MKI67. Data represented as mean \pm STDEV. Statistical differences were noted using * if $p$-value $<0.05$ or ** if $p$ value $<0.01$. Three biological replicates $(\mathrm{n}=3)$ and three technical replicates were used. (B) Alcian

blue staining, (C) Type II collagen immunohistochemistry of the cultures in growth or chondrogenic media. (Scale bar $=100 \mu \mathrm{m})$.

Alcian blue staining revealed clear differences between FBS and SF samples (Figure 6.8.B). In both growth and chondrogenic media, we observed an intense alcian blue staining in stimulatory SF samples when compared to the rest of serums. Similar matrix deposition was observed for FBS, PL and inhibitory SF samples. Comparison between stimulatory SF samples and allograft cartilage 
revealed similarities in the intensity of proteoglycan staining. Moreover, collagen type II specific staining revealed that despite that proteoglycan deposition is achieved using any serum, it does not enhanced collagen type II staining (Figure 6.8.C). When chondrogenic cocktail was added, only inhibitory SF samples had a slight positive matrix. None of the serums had the capability of conserving the same intensity of the allograft cartilage staining.

A

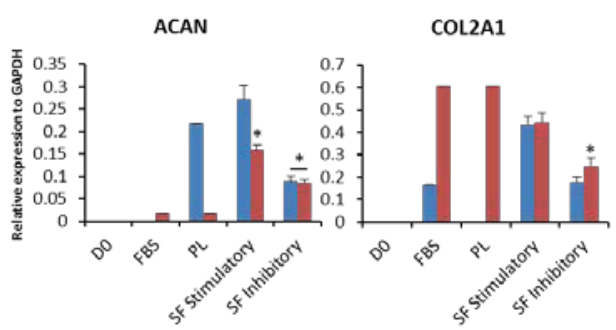

Sox9

MKI67
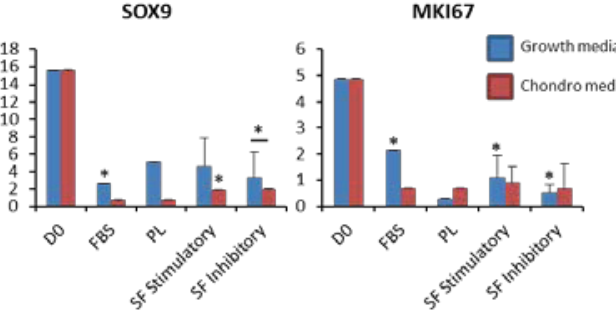

B

Growth media

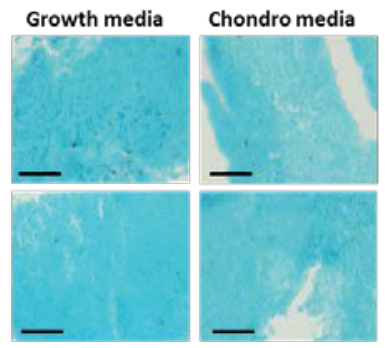

C

Growth media

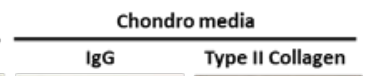

䒢

ֻ
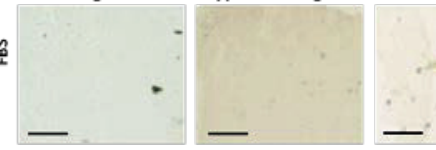

홒

出旁

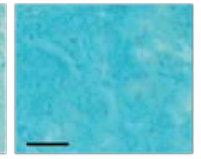

\section{ํํㄹ}

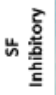
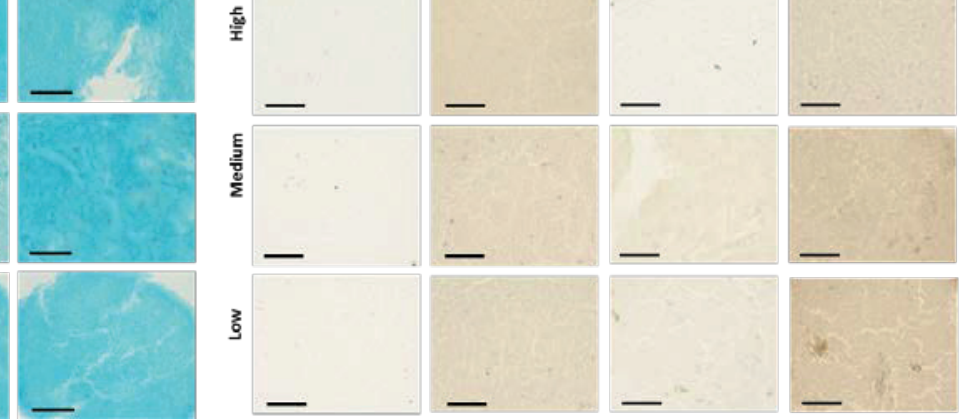

Figure 6.9. aMSC cultured in FBS, PL or SF. Stimulatory or Inhibitory SF samples were used. (A)

Gene expression analysis of cartilage specific markers and proliferation marker MKI67. Data represented as mean \pm STDEV. Statistical differences were noted using * if $p$-value<0.05 or ** if $p$ value $<0.01$. Three biological replicates $(\mathrm{n}=3)$ and three technical replicates were used. (B) Alcian

blue staining, (C) Type II collagen immunohistochemistry of the cultures in growth or chondrogenic media. $($ Scale bar $=100 \mu \mathrm{m})$.

Gene expression of aMSCs chondrogenic differentiation was examined after one week of differentiation (Figure 6.9.A). In growth media, the control serum (e.g. 
PL) promotes the expression of $A C A N$ and SOX9. However, compared to FBS it does not promote COL2A1 and a lower proliferation was observed compared to FBS. Both SF groups were capable of promoting expression of ACAN and collagen type II. But, stimulatory SF samples enhanced the expression of chondrogenic markers COL2A1 and ACAN better than inhibitory samples, FBS or PL. SOX9 expression was steady among the different serums.

Statistical difference reflected the comparison between PL and the other serums. When chondrogenic media is added, a decreased expression in ACAN, SOX9 and MKI67 was observed, whereas collagen type II increased in both SF groups and in FBS. Comparison between inhibitory against stimulatory groups of SF samples revealed stimulatory samples enhanced cartilage markers SOX9 and COL2A1 in a similar way to PL or FBS. Alcian blue staining in both SF groups showed a similar proteoglycan deposition to FBS and PL when chondrogenic media was included (Figure 6.9.B). As observed in the collagen type II immunohistochemistry all SF samples presented low chondrogenic potential whereas FBS shows higher staining of collagen type II when cells were cultured with no chondrogenic cocktail. In the presence of chondrogenic differentiation cocktail both SF groups and FBS showed a similar deposition of collagen type II (Figure 6.9.C). To conclude, viability does not correlate with chondrogenic potential of SF samples. For chondrocytes cultures, stimulatory samples seemed to promote the deposition of proteoglycan and glycosaminoglycan in a better way that inhibitory samples and this was increased by the addition of chondrogenic cocktail. However, stimulatory SF samples had lower potential to deposit collagen type II whereas inhibitory samples seemed to promote collagen type II. Despite our best efforts, neither serum can promote chondrogenesis as good as is seen in the allograft cartilage tissue, but SF samples are safe to use to culture primary chondrocyte and seemed to help the cells maintain their phenotype better than FBS or PL. For AMSCs, SF samples can increase expression of ACAN, collagen type 
II in a similar way that FBS and PL. SF samples from both groups were enough to promote proteoglycan deposition on the aMSC. However, collagen type II matrix produced by these cells was poor. Collagen type II matrix was increased by the addition of chondrogenic media for all serums. SF was capable of producing cartilage matrix with the addition of chondrogenic cocktail similar to no serum conditions with chondrogenic media.

Table 6.5. Literature review of cytokines present during OA progression.

\begin{tabular}{|c|c|c|c|c|}
\hline OA Biomarkers & Official name & Reference & Description & $\begin{array}{l}\text { OA Progression } \\
\text { (H or L })\end{array}$ \\
\hline$I L-1 a$ & $\begin{array}{l}\text { Interleukin } 1 \\
\text { alpha }\end{array}$ & $\begin{array}{l}\text { Tsuchiuda AL et al., } 2014 \text {, } \\
\text { Cleaver CS et al., } 2001\end{array}$ & $\begin{array}{l}\text { Induces collagen release, Role in RA. Invobved in various immune responses, inflammatory processes, and } \\
\text { hematopoiesis. Released in response to cell injury. Induces apoptosis. }\end{array}$ & $\mathrm{H}$ \\
\hline$I L-1 \beta$ & $\begin{array}{l}\text { Interleukin } 1 \\
\text { beta }\end{array}$ & Tsuchiuda AL et al., 2014 & $\begin{array}{l}\text { Role in RA. This cytokine is produced by activated macrophages as a proprotein. Its an important mediator of } \\
\text { the inflammatory response, and is involved in a variety of cellular activities, including cell proliferation, } \\
\text { differentiation, and apoptosis. }\end{array}$ & $\mathrm{H}$ \\
\hline$I L-4$ & Interleukin 4 & Tsuchiuda AL et al., 2014 & $\begin{array}{l}\text { Participates in at least several B-cell activation processes as well as of other cell types (PubMed-3016727). It is } \\
\qquad \text { a costimulator of DNA-synthesis. }\end{array}$ & $\mathrm{L}$ \\
\hline IL-6 & Interleukin 6 & $\begin{array}{l}\text { Tsuchiuda AL et al., 2014, } \\
\text { Acosta JC et al., 2008, Kojima H } \\
\text { et al., 2012, Gobezie R et al., } \\
\text { 2007, Kokebie R et al., 2011, } \\
\text { Scanzello CR et al., 2009 }\end{array}$ & $\begin{array}{l}\text { Secreted by senescent cells, induces DNA damege and telomere shortening. Not produced by cartiage, high in } \\
\text { cartilage defects than in OA chondrocytes, Also role in RA, Levels correlate with radiographic OA, In vitro } \\
\text { stimulation increases TIMP1 And COL2. This gene encodes a cytokine that functions in inflammation and the } \\
\text { maturation of B cells. The protein is primarily produced at sites of acute and chronic inflammation. The } \\
\text { functioning of this gene is implicated in a wide variety of inflammation-associated disease states, including } \\
\text { suspectibility to diabetes mellitus and systemic juvenile theumatoid arthritis. }\end{array}$ & $\mathrm{H}$ \\
\hline IL-7 & Interleukin 7 & $\begin{array}{l}\text { Rubenhagen R et al., } 2012, \\
\text { Tsuchiuda AL ot al., } 2014\end{array}$ & $\begin{array}{l}\text { This cytokine and the hepatocyte growth factor (HGF) form a heterodimer that functions as a pre-pro-B cell } \\
\text { growth-stimulating factor. Hematopoietic gromth factor capable of stimulating the proliferation of lymphoid } \\
\text { progenitors. It is important for proliferation during certain stages of B-cell maturation. }\end{array}$ & $\mathrm{L}$ \\
\hline$I L-10$ & Interleukin 10 & $\begin{array}{l}\text { Tsuchiuda AL et al., 2014, } \\
\text { Papalia R, et al., } 2016\end{array}$ & $\begin{array}{l}\text { The protein encoded by this gene is a cytokine produced primarily by monocytes and to a lesser extent by } \\
\text { lymphocytes. This cytokine hes pleiotropic effects in immunoregulation and inflammation. This cylokine can } \\
\text { block NF-kappa B activity, and is involved in the regulation of the JAK-STAT signaling pattway. }\end{array}$ & $\mathrm{H}$ \\
\hline IL-13 & Interleukin 13 & $\begin{array}{l}\text { Tsuchiuda Al. et al., 2014, Van } \\
\text { Dyken SJ et al., 2013, LafyatisR } \\
\text { et al., 2012, }\end{array}$ & $\begin{array}{l}\text { Higher in cartilage defects, Macrophage activation in tissue homeostasis and repair, Prevents colagen release } \\
\text { This cytokine down-regulates macrophage activity, thereby inhibits the production of pro-inflammatory cytokines } \\
\text { and chemokines. }\end{array}$ & H \\
\hline IL-17 & Interleukin 17 & Papalia R, et al. , 2016 & $\begin{array}{l}\text { The protein encoded by this gene is a proinflammatory cytokine produced by activated T cells. This cytokine } \\
\text { regulates the activities of NF-kappaB and mitogen-activated protein kinases. This cylokine can stimulate the } \\
\text { expression of IL6 and cyclooxygenase-2 (PTGS2/COX-2), as well as enhance the production of nitric oxide } \\
\text { (NO). High levels of this cytokine are associated with seweral chronic inflammatory diseases including } \\
\text { meumatoid arthritis, psoriasis and multiple sclerosis. }\end{array}$ & $\mathrm{H}$ \\
\hline IL-18 & interleukin 18 & Rubenhagen R et al., 2012 & $\begin{array}{l}\text { The protein encoded by this gene is a proinflammatory cytokine that augments natural killer cell activity in spleen } \\
\text { cols, and stimulates interferon gamma production in T-helper type I cells. }\end{array}$ & L \\
\hline IL1Ra & interleukins & Rubenhagen R et al., 2012 & $\begin{array}{l}\text { This protein inhibits the activities of interleukin 1, alpha (IL1A) and interleukin 1, beta (IL1B), and modulates a } \\
\text { varicty of interleukin } 1 \text { related immune and inflammatory responses. }\end{array}$ & $\mathrm{L}$ \\
\hline$T N F a$ & $\begin{array}{c}\text { Tumor necrosis } \\
\text { factor alpha }\end{array}$ & $\begin{array}{l}\text { Tsuchiuda AL et al., 2014, } \\
\text { Papalia R, et al., 2016, Coimbra } \\
\text { JB et al., 2004 }\end{array}$ & $\begin{array}{l}\text { Also role in RA, If TNFa is produced also IL8 and IL1 are up regulated. Mainly secreted by macrophages. It can } \\
\text { bind to, and thus functions through its receptors TNFRSF 1AVTNFR1 and TNFRSF1B/TNFBR. involved in the } \\
\text { regulation of cell proiferation, differentiation, apoptosis, lipid metabolism, and coagulation. This cytokine has } \\
\text { been implicated in a variety of diseases, including autoimmune diseases, insulin resistance, and cancer. }\end{array}$ & $\mathrm{H}$ \\
\hline IFNY & Interferon & Tsuchiuda AL ot al., 2014 & $\begin{array}{l}\text { Higher in cartilage defects. Triggers a cellular response to viral and microbial infections. It is a potent activator of } \\
\text { macrophages, it has antiproliferatwe effects on transformed cells and it can potentiate the antiviral and antiumor } \\
\text { effects of the type I interferons. }\end{array}$ & $\mathrm{H}$ \\
\hline OSM & Oncostatin $M$ & $\begin{array}{l}\text { Tsuchiude AL et al., } 2014, \\
\text { Cleaver CS et al., } 2001\end{array}$ & $\begin{array}{l}\text { Induces collagen release, Not produced by cartilage. This protein is a secreted cytokine and growth regulator } \\
\text { that inhibits the proliferation of a number of tumor cel lines. This protein also regulates the production of other } \\
\text { cytokines, including interleukin } 6 \text {, granulocyte-colony stimulating factor and granulocyte-macrophage colony } \\
\text { stimulating factor in endothelial cells. }\end{array}$ & $H$ \\
\hline
\end{tabular}

\subsubsection{Cytokine analysis.}

Analysis of the cytokine composition was performed to optimize samples for culture conditions. First, a literature review was performed to examine a list of cytokines present in cartilage OA compared to healthy cartilage (Table 6.5). 
Tsuchiuda et al. examined the presence of cytokine in synovial fluid from cartilage defect and OA cartilage and found that IL1 $\beta$, IL10, IL6 and TNF $\alpha$ were significantly up-regulated in OA compared to cartilage defects [12]. They found these cytokines to be expressed in expanded OA chondrocyte in vitro. A subset of the samples was examined for ELISA analyses of these 4 cytokines. For reliability testing, we analyzed each sample and standard curve controls in duplicate. We observed that all 24 samples were positive for the three of the four cytokines; however, some differences between the samples were also observed (Figure 6.10). Moreover, we detected that even in undiluted samples the intrinsic levels of IL1 $\beta$ were low in comparison to other cytokines (e.g. IL6 and IL10) levels. Therefore, the collected samples may not express IL1 $\beta$.

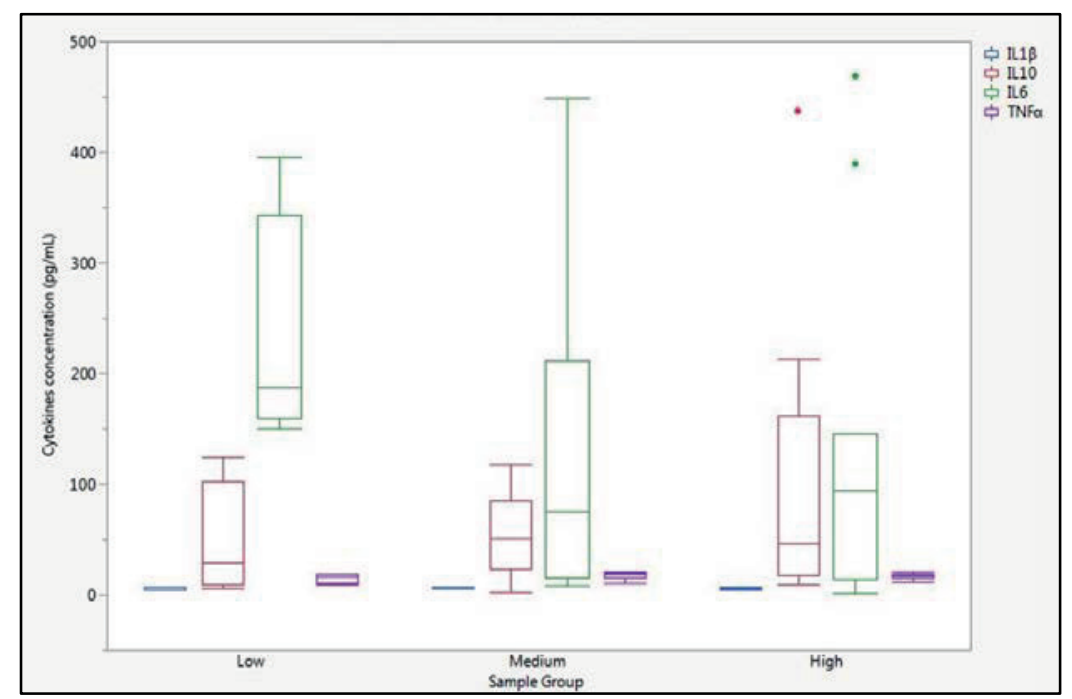

Figure 6.10. Boxplot demonstrating the difference in concentration of synovial fluid proinflammatory cytokines IL1 $\beta$, IL10, IL6 and TNF $\alpha$.Values are presented as median (line), interquartile range (box) and outliers (circles). Statistical differences were noted using * if $p$-value $<0.05$.

A non-parametric comparison for all pairs using Steel-Dwass Method was performed. The median and 95\% confidence intervals (CI) for each cytokine were described below. For IL1 $\beta$ the median was $6.28 \pm 0.86 \mathrm{pg} / \mathrm{mL}$ with a lower 
CI of $6.65 \pm 0.67$ and an upper CI of $6.65 \pm 1.21$. For IL10 the median was $74.46 \pm 93.26 \mathrm{pg} / \mathrm{mL}$ with a lower $\mathrm{CI}$ of $35.08 \pm 72.48$ and an upper CI of $113.84 \pm 130.81$. For IL6 the median was $143.87 \pm 145.94 \mathrm{pg} / \mathrm{mL}$ with a lower CI of $82.25 \pm 113.42$ and an upper CI of $205.49 \pm 204.71$. For TNF $\alpha$ the median was $16.75 \pm 3.24 \mathrm{pg} / \mathrm{mL}$ with a lower CI of $15.38 \pm 2.51$ and an upper CI of $18.11 \pm 4.54$. IL6 was inversely associated with protein content in the samples. In contrast, IL10 was highly increased in samples with higher protein content. IL1 $\beta$ and TNF $\alpha$ content were similar among the samples. However, a slight increase of both cytokines was found in medium protein content samples. Thus, SF samples with lower protein content possessed more inflammatory cytokines than the other two SF groups. Interestingly, the low protein content samples were previously found to impair cell viability more than SF with higher protein content.

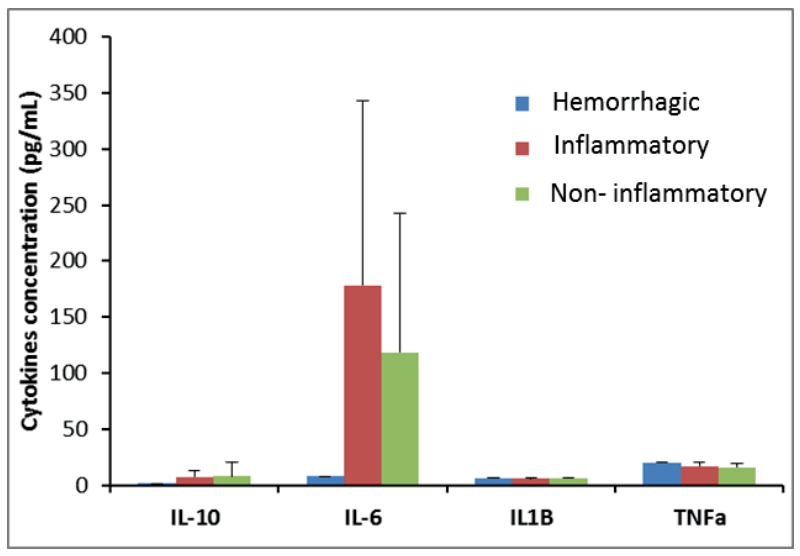

Figure 6.11. Cytokine concentrations categorized according to visual inspection of clarity of SF. Values are presented as mean \pm STDEV. Statistical differences were noted using * if $p$ value $<0.05$.

In Figure 6.11, cytokine categorized according to visual inspection of the clarity of the fluid validated previous observations of no variability and low concentrations of IL1 $\beta$ and TNF $\alpha$. However, both IL10 and IL6 were found increased in samples that presented a non-inflammatory and inflammatory 
aspect. Thus, low protein SF samples presented a visual aspect of OA inflammatory and higher cytokine markers. These samples were shown to improve viability of aMSC, but decreased viability of human chondrocyte.

\subsection{DISCUSSION}

Fetal bovine serum (FBS) has long been the gold standard medium supplement for laboratory-scale MSC culture and other cells. However, FBS has a poorly characterized composition and poses risk factors, as it may be a source of xenogenic antigens and zoonotic infections [13]. FBS is rich in fetal growth factors and hormones that stimulate cellular proliferation and maintenance; and it facilitates differentiation toward various lineages [14]. The right choice of serum or serum replacement is crucial for cell culture. Particularly with regard to clinical application, human alternatives for fetal bovine serum are clearly to be preferred.

Scientists and users involved in cell therapy or advanced therapy medicinal products know the need for an alternative. Since concerns have been raised regarding the safety of FBS, other serums have been tested and used with some success [15]. Each type of culture supplement has advantages and disadvantages, and they carry severe effects on functionality and viability of the cells. Use of platelet lysate was studied and used to culture a broad range of different cell types. Long-term culture of MSCs in media supplemented either with FBS or pooled PL induced similar gene expression and DNA methylation changes in expanded cells [16]. The use of PL-supplemented medium generates significantly more MSCs than the use of fetal bovine serum. Thus, platelet lysate can be an adequate non-xenogenic alternative for fetal calf serum. However, PL also triggers an inflammatory response in MSCs and induces the secretion of factors maintaining macrophages in a proinflammatory state [17].

Several studies determined that the overall protein content of PL across different batches was highly similar (40-60 g/L) but the growth factor content differed 
substantially. These studies also used proteomics to illustrate the impact of different serum compositions during cell expansion. In our study, SF was used as an alternative for both FBS and PL. SF rinses all intrinsic structures of joints. It's used in the evaluation of joint diseases and is been broadly used for the analysis of its constituents to understand OA. Moreover, SF can be a promising growthstimulating additive for culturing of stem cells and other cell types. For stem cell delivery therapies, the importance of synovial fluid characterization lies in the fact that this fluid interacts with the cells once implanted. Thus, it is important to know if synovial fluid could enhance, limit or reject stem cell or chondrocyte upon implantation.

Our data indicates that synovial fluid can enhance the metabolic activity of chondrocyte and aMSC at a level that is at least equivalent to FBS and better than human alternative PL. Also, this data shows that proliferation seemed to correlate with protein content and pathology of the sample. Synovial fluid with medium protein content may be likewise effective during in vitro expansion and in promoting chondrocytic phenotype maintenance in human chondrocyte than fetal bovine serum. Moreover, synovial fluid supplemented with chondrogenic media in aMSC slightly promoted proteoglycan deposition after 21 days in culture. However, due to the differences found between the SF samples during cell culture, further normalization of the samples will be essential to preserve both stem cells and chondrocytes viability and maintenance.

Concentrations of synovial fluid protein are known to vary with the degree of joint inflammation. Comparing the protein profile to determine potential key components that could influence cell culture is important for the normalization of the synovial fluid. Proteomic analysis has been previously used as an analytical tool to detect biomarkers for disease. Also, these cytokine profile are different between synovial fluid, cartilage tissue and cultured chondrocytes [18]. IL6 has been observed to stimulate the expression of MMPs that act to degrade cartilage 
[19]. IL1 $\beta$ and TNF $\alpha$ presence has been shown to inhibit chondrogenic differentiation of stem cells by blocking TGF $\beta$ signaling and SOX9 expression[20]. In our dataset, both IL1 $\beta$ and TNF $\alpha$ expression were low and similar across the samples. However, in the case of IL6 higher expression was found in low protein content samples. Interestingly, these samples affected the viability of both aMSC and human primary chondrocyte. Thus, correlation between viability, protein content and cytokines profile could help for the stratification of samples for future therapeutic use.

Our results serve as an initial screening of the possibilities of SF to replace fetal bovine serum as a culture supplement for in vitro expansion of human primary chondrocyte and aMSC. Due to its non-zoonotic (animal free) nature is therefore potentially better for clinical applications. However, compared to FBS and PL, synovial fluid cannot be obtained in large quantities, which would make it difficult to be used.

In conclusion, this study suggested that in clinical therapies where aMSC are injected into a diseased joint, the presence of a synovial fluid rich environment would not affect the viability of the aMSC implanted. Thus, the existence of OA SF does not significantly disrupt cell number and/or proliferation. Increasing the potential to conserve an appropiate cell number to perform the expected function. Future studies will aim to analyze the precise composition of synovial fluid (e.g. growth factors, microRNAs and exosomes) to optimize and define culture conditions. 


\section{REFERENCES}

1. Oatis CA: Kinesiology. The mechanics and pathomechanics of Human Movement. 2003.

2. Ritter S, Subbaiah R, Bebek G, Cirsh J, Scanzello Cr, Krastins B, Sarracino D, Lopez MF, Crow MK, Aigner T, Goldring MB, Goldring SR, Lee DM, Gobezie R, Aliprantis AO: Proteomic analysis of synovial fluid from the osteoarthritic knee: comparison with transcriptome analyses of joint tissues. Arthritis Rheum 2013:981-992.

3. Steele B, Alvarez-Veronesi M, Schmidt T: Molecular weight characterization of PRG4 proteins using multi-angle laser light scattering (MALLS). Osteoarthritis Cartilage 2013:498-504.

4. Petty R, Wedderburn L: Textbook of Pediatric Rheumatology (Seventh Edition). 2016.

5. Stellavato A, De Novellis F, Reale S, De Rosa M, Schiraldi C: Hybrid complexes of high and low molecular weight: evaluation using an in vitro model of osteoarthritis. J Biol Regul Homeost Agents 2016:7-16.

6. Clinical Trials: Joint Disease [https://stemcellsportal.com/clinical_trials_joint_disease].

7. Nečas D, Vrbka M, Galandáková A, Křupka I, Hartl M: On the observation of lubrication mechanisms within hip joint replacements. Part I: Hard-on-soft bearing pairs. Journal of the Mechanical Behavior of Biomedical Materials 2018.

8. Waller K, Chin K, Jay G, Zhang L, Teeple E, McAllister S, Badger G, Schmidt T, Fleming B: Intra-articular Recombinant Human Proteoglycan 4 Mitigates Cartilage Damage After Destabilization of the Medial Meniscus in the Yucatan Minipig. Am J Sports Med 2017:1512-1521.

9. Walker HK, Hall Wd, Hurst JW: Clinical Methods: The History, Physical, and Laboratory Examinations. The Joint Fluid. 3rd edition. Edited by Walker HK HW, Hurst JW, Editors. Boston: Butterworths; 1990

10. Dudakovic A, Camilleri E, Riester SM, Lewallen EA, Kvasha S, Chen X, van Wijnen AJ.: High-resolution molecular validation of self-renewal and spontaneous differentiation in clinical-grade adipose-tissue derived human mesenchymal stem cells. J Cell Biochem 2014:1816-1828.

11. Leijten JC, Bos JS, Landman EB, Georgi N, Jahr H, Meulenbelt I, Post JN, van Blitterswijk CA, Karperien M.: GREM1, FRZB and DKK1 mRNA levels correlate with osteoarthritis and are regulated by osteoarthritis-associated factors. Arthritis Res Ther 2013:15(15): R126.

12. Tsuchiuda A, Beekuizen M, Hart M, Radstake T, Dhert W, Saris D, van Osch G, Creemers L: Cytokine profiles in the joint depend on pathology, but are different between synovial fluid, cartilage tissue and cultured chondrocytes. Arthritis Research \& Therapy 2014:16:441.

13. Evaluation of human platelet lysate versus fetal bovine serum for culture of mesenchymal stromal cells. Cytotherapy 2014:170-180.

14. Gospodarowicz D, Ferrara N, Schweigerer L, Neufeld G: Structural characterization and biological functions of fibroblast growth factor. Endocr Rev 1987:95-114.

15. Heldin C, Betsholtz C, Johnsson M, Nister M, Ek L: Platelet-derived growth factor: mechanism of action and relation to oncogenes. J Cell Sci Suppl 1985:65-76. 
16. Shanskii Y, Sergeeva N, Sviridova I, Kirakozov M: Human Platelet Lysate as a Promising Growth-Stimulating Additive for Culturing of Stem Cells and other Cell Types. Bulletin of Experimental Biology and Medicine 2013:146-151.

17. Ulivi V, Tasso R, Cancedda R, Descalzi F: Mesenchymal Stem Cell Paracrine Activity Is Modulated by Platelet Lysate: Induction of an Inflammatory Response and Secretion of Factors Maintaining Macrophages in a Proinflammatory Phenotype. Stem Cells and Development 2014.

18. Liao W, Li Z, Zhang H, Li J, Wang K, Yang Y: Proteomic analysis of synovial fluid as an analytical tool to detect candidate biomarkers for knee osteoarthritis. Int J Clin Exp Pathol 2015:9975-9989.

19. Leung Y, Huebner J, Haaland B, Wong S, Kraus V: Synovial fluid pro-inflammatory profile differs according to the characteristics of knee pain. Osteoarthritis Cartilage 2017:1420-1427.

20. Kondo M, Yamaoka K, Tanaka Y: Acquiring Chondrocyte Phenotype from Human Mesenchymal Stem Cells under Inflammatory Conditions. Int J Mol Sci 2014:2127021285. 


\section{CHAPTER 7}

7. Novel transcription factor ZNF648 might regulate cartilage development and homeostasis through the modulation of cartilage related signaling pathways. 


\section{ABSTRACT}

RNA-seq analysis of mesenchymal tissues shows a unique expression of ZNF648 in all cartilaginous tissues like the growth plate, articular cartilage, chondrosarcoma and intervertebral disc compared to non-cartilaginous musculoskeletal tissues. Primary growth plate cells showed a higher expression of this transcription factor when compared to primary articular chondrocytes. In addition, ZNF648 expression was also found increased in moderate diseased cartilage tissues. This ZNF648 protein in the growth plate seems to maintain normal differentiation of pre-committed chondrogenic mesenchymal progenitor cells (e.g., human growth plate and immature mouse chondrocytes) into chondrocytes, whereas in later stages of cartilage maturation ZNF648 expression is reduced. Although the expression is present in primary tissues, it is significantly reduced when cells are removed from the native tissue environment. A reduction in ZNF648 expression was found in primary cells passaged two times and on in vitro cell cultures of primary cells. We used in vitro cell culture, gain and loss of function analysis and a Zfp648 null mouse to understand the role of ZNF648 during cartilage development. Interestingly, we found that ZNF648 may regulate different programs in articular and growth plate chondrocytes. An enhanced expression of ZNF648 increased COL2A1 expression in growth plate cells while reducing the expression in articular chondrocytes cells. This suggested an important regulatory mechanism during early development of the chondrocytes in the growth plate and not in terminally differentiated chondrocytes such as the ones in articular cartilage. Similarly, knock down experiments validated the regulatory role of ZNF648 or Zfp648 in enhancing collagen type II and decreasing collagen type I. This concept would be ideal for formation of hyaline cartilage over fibrocartilage in tissue regeneration strategies. Lastly, Zfp648 null mice obtained using CRISPR/Cas9 strategies showed skeletal differences in younger mice (e.g. 2 weeks, 3 weeks) whereas in skeletal mature mice (e.g. 12 weeks of age) no phenotype was observed. 
Remarkably, the phenotype obtained from 2-week-old null mouse indicates an important role of this transcription factor in the development of long bones and during endochondral ossification stages. Zfp648 depletion not only affected the onset of the formation of secondary ossification center but also it delayed the formation of normal long bones. In this study we aimed to analyze driving molecular factors such as transcription factor ZNF648 that may directly regulate cartilage development or homeostasis through modulating gene expression and other cartilage-related signaling pathways. Thus, development of novel approaches to improve cartilage regeneration from a mechanistic and molecular point using ZNF648 to maintain cartilage homeostasis is thought to play and essential role in future clinical therapies. 


\subsection{INTRODUCTION}

The growth plate is a complex cartilaginous structure that critically regulates natural growth of long bones and the axial skeleton. It is a highly specialized cartilaginous tissue adjacent to the joint in skeletally immature vertebrates. Genetic disorders, infection, tumor, and traumatic injuries to the growth plate can result in shortening and progressive angular deformity of the affected limb in the growing child [1, 2]. Pediatric limb length discrepancy and angular deformities are clinically challenging problems that cost an estimated $\$ 2.2$ billion dollars in treatment each year in the United States [3]. Each year, one in three hundred U.S. children sustains a growth plate fracture, and four percent will sustain permanent damage to the growth plate resulting in deformity or shortening [4]. This damage could impact later function and quality of life.

Without appropriate treatment, growth plate injuries result in lifelong limp, chronic pain, arthritis, and disability. However, there are no pharmacotherapies available for local or selective treatment of growth plate disorders, leaving mechanical treatments as the only available option [5]. Mild deformities may be corrected by surgical tethering [6]. Though, for correcting leg length discrepancy, this often entails performing surgery on the normal leg to destroy the healthy growth plate, resulting in overall decreased stature in adulthood. Unfortunately, these techniques do not work at all if the child is too close to skeletal maturity. For severe deformities, shortening the longer leg is not feasible because it would result in severe secondary deformity and dysfunction. Thus, complex surgical reconstruction is undertaken using external fixation devices or intramedullary lengthening nails [7]. Although the process requires months of rehabilitation, the lengthening still takes place too quickly for the human body to adapt, resulting in various adverse events for the patients. Even after completion of the treatment, fracture is common because the regenerated bone is not robust [8]. Thus, there is a significant need for improved understanding of growth plate signaling 
pathways to reduce surgical morbidity and provide local pharmacologic treatment options.

A comprehensive biologic approach to the treatment of the physis is needed in order to restore normal growth, decrease morbidity, and improve patient outcomes. Local pharmacologic treatment to promote physeal growth could provide slow, gradual correction of limb deformities without associated comorbidities and prolonged recovery. Reversible and controllable agents could be used to provide precision correction of an individual's deformity, mitigating the risk of over- or under-correction. Moreover, these interventions could encourage growth in the short limb and suppress growth in the long limb avoiding shortening stature in the adulthood. However, a lack of robust data and scientific knowledge of the physis has delayed the development of local pharmaceuticals to treat growth plate disorders. Hence, a better understanding of the regenerative properties of the physis also holds promise to regenerate not only the growth plate but to treat other disabling problems of the musculoskeletal system. Our overall goal is to elucidate the mechanism of transcription regulation in musculoskeletal tissues. RNA-seq analysis of mesenchymal tissues was examined to identify expression of ZNF648 in all cartilaginous tissues like growth plate, articular cartilage, chondrosarcoma and intervertebral disc and compared to non-cartilaginous musculoskeletal tissues. Expression of ZNF648 will be also examined in tissues, primary cell digestions and immortalized cells from human and mouse. Differences in gene expression and protein would be identified. Functional analysis on different cells will be performed with the goal of obtaining information about the mechanistic effects of gain and loss of function of ZNF648 in cartilage. Moreover, a Zfp648 null mouse obtained using CRISPR/Cas9 strategies will be studied to observe skeletal phenotypic differences. In this study we aimed to analyze driving molecular factors such as transcription factor ZNF648 that may directly regulate cartilage development or homeostasis 
through modulating gene expression and other cartilage-related signaling pathways.

\subsection{MATERIALS AND METHODS}

\subsubsection{RNA extraction from tissues}

More than 1200 tissue specimens were collected from patients undergoing tissue removal as part of planned surgical procedures. The specimens used in this investigation were collected under Institutional Review Board (IRB) of Mayo Clinic. Written informed consent was obtained for all collected tissues. Some cartilage samples were collected from human cadaver donors through the Gift of Hope Organ and Tissue Donor Network (Elmhurst, IL), with approval by the local ethics committee and informed consent from each family (ORA\#: L03090306). Before RNA extraction, samples were snap frozen in liquid nitrogen and stored at $-80^{\circ} \mathrm{C}$ after surgical harvest. Then, frozen tissues were grounded into a powder using a mortar and pestle and homogenized in Qiazol reagent (Qiagen, Hilden, Germany). Total RNA was extracted from using the RNeasy minikit (Qiagen, Hilden, Germany) and quantified using a NanoDrop 2000 spectrophotometer (Thermo Fischer Scientific, Wilmington, Delaware). Before RNA extraction, articular cartilage specimens were classified according to radiological findings using the Collins grading scheme as described previously [9]. For this study, joints were specifically classified using the following criteria: G0 joints displayed normal articular cartilage throughout $(n=5)$, G1 joints had some destruction of superficial cartilage and swelling and/or softening ( $n=7)$, G2 joints exhibited extensive superficial damage to articular cartilage but not significant loss of cartilage and no damage to bone (n=6), G3 joints had areas with total articular cartilage loss with observable penetration of defects to the subchondral bone plate $(n=4)$, and a G4 joint was identified as having complete loss of cartilage and full thickness exposure to bone along the articular surface of the joint. 


\subsubsection{Next generation RNA sequencing}

RNAseq analysis was performed using the musculoskeletal tissue collection collected in Dr. van Wijnen's laboratory. We analyzed and compare musculoskeletal tissues and cell cultures of cartilage, physis, bone, tendon, muscle and ligament from different states of disease, and healthy adult and pediatric patients. For samples selected for next generation sequencing, RNA integrity was assessed using the Agilent Bioanalyzer DNA 1000 chip (Invitrogen, Carlsbad, CA). Only samples with an RNA Integrity Number (RIN) and DV200 score greater than our Sequencing Core's minimum cutoff (RIN $>6$ and DV200 > 50\%) were used for sequencing. High-throughput RNA sequencing and subsequent bioinformatic analysis were performed in collaboration with the Mayo Clinic RNA sequencing and bioinformatics cores as previously described [10]. Library preparation was performed using the TruSeq RNA library preparation kit (Illumina, San Diego, CA). Poly-A mRNA was purified using oligo dT magnetic beads. Then, cDNA synthesis was performed on purified mRNA using SuperScript III reverse transcriptase and random primers (Invitrogen). DNA fragments were enriched by PCR using primers included in the Illumina Sample Prep Kit. Sample concentration and size distribution was determined on an Agilent Bioanalyzer DNA 1000 chip and Qubit fluorometry (Invitrogen, Carlsbad, CA). RNA-Seq data was analyzed using the MAPRSeq v.1.2.1 system including alignment with TopHat 2.0.6 [11] and HTSeq software [12]. Expression values for each gene were normalized to 1 million reads and corrected for gene length (reads per kilobasepair per million mapped reads, RPKM).

\subsubsection{Human cell harvest and expansion}

Human primary chondrocyte were obtained from healthy donors undergoing amputation procedures for congenital limb deformity or from donors undergoing total knee replacement surgery. All zones of growth plate and articular cartilage were used. All tissues and cells were collected under protocols approved by the Mayo Clinic Institutional Review Board. After surgical harvest, both articular 
cartilage and growth plate specimens were immediately placed in a solution of 2\% Pronase (Sigma Aldrich) in culturing media (DMEM supplemented with 1\% anti-mycotic/antibiotic) for 1 hour in a shaking incubator at $37^{\circ} \mathrm{C}$. Then, samples were incubated overnight in a $0.36 \%$ collagenase-P (Worthington) solution in culturing media in a shaking incubator at $37^{\circ} \mathrm{C}$ overnight. Undigested tissue was removed by filtering the cell suspension with a cell strainer. Cells were centrifuged and washed in two changes of PBS. Lastly, chondrocytes were resuspended in maintenance media that contained Advanced MEM (Gibco/Thermo Fisher Scientific) supplemented with $10 \%$ fetal bovine serum (Atlanta Biologicals, Atlanta, GA) and 1\% penicillin/streptomycin (Gibco/Thermo Fisher Scientific).

A comparison between the passaging of cells was performed. Passage 0 (primary digested cells), passage 1 and passage 2 cells were cultured using standard techniques in $10 \mathrm{~cm}^{2}$ dish at $37^{\circ} \mathrm{C}, 95 \%$ humidity and $5 \% \mathrm{CO}_{2}$ until they reached $80 \%$ confluence. Prior to each passage, cells were detached from the $10-\mathrm{cm}^{2} \mathrm{dish}$ by trypsinization using TrypLE Express (Gibco/Thermo Fisher Scientific) and replated under the same standard conditions. RNA was extracted from cells at passage 0 (primary digested cells), passage 1 and passage 2 for gene expression analysis.

For time course (day 1, day 3 and day 7) culture experiments, primary articular cartilage chondrocyte from healthy and OA patients, and primary growth plate chondrocyte were plated in high-density cellular aggregates as described previously [13]. Cells were cultured for one week in maintenance media and media was refreshed every 2 to 3 days. RNA was extracted from cells after 1,3 and 7 days for gene expression analysis.

\subsubsection{Isolation, culture and analysis of immature mouse chondrocytes (mACs)}

Primary immature mouse articular chondrocytes chondrocytes (mACs) were isolated from post-natal day 7 old mice as previously described by Camilleri et al. 
[14]. Freshly isolated mACs were re-suspended in at a concentration of $2 \times 10^{7}$ cells/mL in maintenance media (DMEM supplemented with 2\% FBS and 1\% antimycotic/antibiotic) and plated in micromass culture by pipetting $10 \mu \mathrm{L}$ of cell suspension on to a low-adhesion tissue culture dish. Micromasses formed by incubating for $1 \mathrm{~h}$ at $37^{\circ} \mathrm{C}, 5 \% \mathrm{CO}_{2}$, after which growth medium was carefully added and left undisturbed for 7 days. Before RNA extraction at day 7, micromasses were washed with PBS to remove medium, mechanically disrupted with a scalpel, and $700 \mu \mathrm{L}$ of QIAzol was added to lyse cells. After RNA was extracted gene expression analysis was performed.

\subsubsection{Cell culture and expansion of immortalized cells lines}

Human and mouse immortalized cell lines were used as positive and negative controls in our studies. Two different human cell lines were used, TC28A2 and chondrosarcoma cell line L835, whereas one mouse immortalized cell line ATDC5 was utilized. TC28A2 human chondrocyte cell lines (Sigma) and ATDC5 mouse chondrogenic cell line (Sigma) were both expanded using Advanced MEM supplemented with $10 \%$ fetal bovine serum and 1\% penicillin/streptomycin and cultured in standard conditions. Chondrosarcoma cell line L835 was obtained from the University of Leiden and cultured as previously described [15]. HEK293 cells were cultured and expanded using maintenance media, which consisted of DMEM with 10\% FBS and 1\% anti-mycotic/antibiotic under standard conditions. When $80 \%$ confluency was achieved, cells were detached from the flasks and quantified before experimental cultures were performed.

\subsubsection{Functional analysis of ZNF648}

Gain and loss of function experiments were performed in human and mouse cells. Before transfection, all cells were seeded in 6-well plates with at a density of 10,000 cells per $\mathrm{cm}^{2}$. Cells were cultured in maintenance media (specific for each cell) until $80 \%$ confluency was reached. 
Dose response experiments were performed using HEK293 cells. For this, 2, 4 and $5 \mu \mathrm{g}$ of an ZNF648 plasmid tagged with a GFP or mCherry protein was used (RFP-ZNF648 plasmid). Transfection was performed using Lipofectamine following the manufacturer's instructions. A negative plasmid only containing GFP or mCherry tag was used as a negative control. After 72 hours of transfection, ZNF648 expression was analyzed using fluorescent microscopy analysis and western blotting. RNA was collected from the cells and gene expression analysis was performed. Metabolic assay and Hoechst staining was performed to analyze the toxicity of the transfected plasmid after 24 and 72 hours of transfection.

Gain and loss of function experiments were performed on primary and immortalized chondrocytes. For gain of function, transfection was performed using the Lipofectamine protocol and adding $5 \mu \mathrm{g}$ of each plasmid to each well. For loss of function experiments 10nM of ZNF648 siRNA for human cells or 10nM of Zfp648 siRNA for mouse cells was used following Lipofectamine manufaturer's instructions. After 72 hours of transfection, gene expression, protein analysis and fluorescent imaging were performed. MTS activity assay was performed after 24 hours of transfection.

\subsubsection{RNA isolation, reverse transcription and real time quantitative PCR (RT-qPCR)}

For all experiments, total RNA was isolated using the Direct-zol RNA kit (ZYMO Research, Irvine USA) following the instructions of the manufacturer. Three different biological replicates for each experimental condition were used concurrently for RNA isolation and subsequent expression analysis, to ensure consistency in RNA quality and expression values. Then, RNA concentrations and purity levels were measured using a NanoDrop (Thermo Fisher Scientific). For reverse transcription, $1 \mu \mathrm{g}$ of RNA was used as template. The Reverse Transcription System using M-MLV Reverse Transcriptase (Promega, Madison, USA) was used to convert RNA to cDNA. Real-time PCR reactions were composed 
of 10 ng of cDNA, gene-specific primers (Table 7.1) and 1x QuantiTect SYBR $®$ Green PCR Master Mix (Qiagen) in a final volume of $10 \mu \mathrm{L}$. RT-qPCR was detected using the CFX384 real time system machine (Bio-Rad). Relative transcript abundance of genes of interest was normalized to AKT1 expression using the 2$\triangle \triangle \mathrm{Ct}$ method. Gene expression analysis of chondrogenic markers (e.g. COL2A1, PRG4, ACAN, SOX9, and SOX6), proliferative markers (e.g. HIST2H4, MKI67 and CCNB2) and ZNF648 was performed. For mouse cells, ZNF648 mouse homolog Zfp648 primer was used.

\subsubsection{Western Blotting}

Total protein was harvested from all different cells after 72 hours of transfection. Cells were washed twice with ice cold PBS and mechanically disrupted using a cell scraper, in the presence of immunoprecipitation buffer $(150 \mathrm{mM} \mathrm{NaCl}, 50 \mathrm{mM}$ Tris $\mathrm{pH} 7.4,1 \% \mathrm{w} / \mathrm{v}$ sodium deoxycholate, $0.1 \% \mathrm{w} / \mathrm{v}$ sodium dodecyl sulfate and $1 \% \mathrm{v} / \mathrm{v}$ Triton X-100) supplemented with protease inhibitor cocktail (Sigma), and PMSF (Sigma) and stored at $-80^{\circ} \mathrm{C}$ until quantification. Protein content was quantified using the DCTM protein assay (Bio-Rad) according to the manufacturer's protocol. Western blotting and membrane development was performed as previously described [16]. The following primary antibodies were used to detect proteins: rabbit polyclonal anti-ZNF648 (1:200; 170269; Abcam), rabbit polyclonal anti-ZNF648 (1:400; AP54705PU-N; Origene), mouse monoclonal anti-COL2A1 (1:500; MAB8887; Millipore) and rabbit monoclonal anti-GAPDH (1:1000; D16H11; Cell Signaling).

\subsubsection{MTS activity assay and Hoechst Staining}

MTS activity was assayed after 24 and 72 hours according to the manufacturer's protocol (Promega). Absorbance was measured at $490 \mathrm{~nm}$ using a SpectraMAX Plus spectrophotometer (Molecular Devices). Then, MTS solution was aspirated from wells, and cells were washed once with PBS. After aspiration of PBS, $500 \mu \mathrm{l}$ of Hoechst 33258 (Sigma, 94403) (0.5 $\mu \mathrm{l} / \mathrm{ml}$ in PBS) was added to each well. 
Plates were left to incubate in the dark at room temperature for $20 \mathrm{~min}$. After incubation, fluorescence intensity was measured at a 340-nm excitation wavelength and 460-nm emission wavelength using an F200 Infinite Pro (Tecan) plate reader. Measurements were fit to a standard DNA curve to determine relative DNA content.

\subsubsection{Histological analysis of human and mouse tissues}

Histological analysis was performed on articular cartilage, growth plate and epiphyseal bone. After surgical harvest, tissues were washed with saline solution and immediately fixed in 10\% neutral buffered formalin for one day. Then, samples were dehydrated in graded ethanol and paraffin embedded. Immunohistochemistry (IHC) was performed on serial sections of $5 \mu \mathrm{m}$ thickness using the Mouse and Rabbit Specific HRP (ABC) Detection IHC kit (Abcam) and DAB Enhanced Liquid Substrate System (Sigma-Aldrich) according to instruction manuals. Primary antibodies used were rabbit polyclonal anti-ZNF648 (1:500; 170269; Abcam), mouse monoclonal anti-COL2A1 (1:40; MAB8887; Millipore), rabbit monoclonal anti-COL1A1 (1:100; 138492; Abcam), mouse monoclonal anti-MKI67 (1:100; 238019; Abcam), rabbit monoclonal anti-mouse IgG (1:100; 190475; Abcam) and goat anti-rabbit IgG (1:100; 205718; Abcam).

\subsubsection{Immunofluorescence}

Cells were cultured in a density of $1 \times 10^{4}$ cells per slide in standard conditions. Before fixation, slides were washed thrice with PBS followed by addition of $4 \%$ paraformaldehyde in PBS for 10 minutes at room temperature. Then, glass slides were immersed in PBS containing 0.1\% Triton X-100 for 10 minutes at room temperature. For protein block, PBS containing 5\% BSA was used and slides were blocked for 2 hours at room temperature. After washing steps with PBS, specific primary antibodies were added and incubated overnight in the cold room. The primary antibodies used included rabbit polyclonal anti-ZNF648 (1:500; 170269; Abcam) and mouse monoclonal anti-COL2A1 (1:500; MAB8887; 
Millipore). After washing steps, secondary antibodies were added to the slides. Goat anti-rabbit IgG Alexa Fluor 647 (1:500; abcam; 150083) and goat antimouse IgG Alexa Fluor 488 (1:500; abcam; 150113) were incubated for 30 minutes at room temperature in the dark. Glass slides were then mounted into microscope slides using VECTA shield with DAPI (Vector laboratories) mounting media. Images were digitally photographed using the AxioVert A1 (Zeiss) and Zen software package (Zeiss).

\subsubsection{Genetic null mutation of ZNF648 in mouse and evaluation of skeletal phenotyping}

A Zfp648 global knockout mouse model was generated with the expertise of the Mayo Clinic Transgenic and Knockout Core Facility. The mouse Zfp648 locus was ablated using a Crispr/Cas-9 strategy that targeted the Zfp648 exon using two guide sequences: GGTAGGAAGGGTGGCTCCCC (gRNA1), and CTGAGTGTCAAACTGCGAGT (gRNA2) and generated a deletion of 288bp around the transcriptional start site (TSS) (Figure 7.1.A). Six generations of the offspring from the founders were paired with $\mathrm{C} 57 \mathrm{BL} / 6$ mice in order to eliminate off target effects of the Crispr/Cas-9 gene targeting technique (Figure 7.1.B). Gene knockout and germline transmission of the null allele was confirmed by DNA sequencing and PCR with allele specific primers of generation F1 mice (Figure 7.1.C). For experimentation, an analysis of all three genotypes: knockout (KO; Zfp648 -/-), Wild Type (WT; Zfp648 +/+) and Heterozygous (HET; Zfp648 +/-) was performed.

Skeletal phenotype of the mouse was studied using microCT, histology and immunohistochemistry of growth plate, cartilage and bone. Radiographic analysis was performed using a Faxitron X-ray imaging cabinet (Faxitron Bioptics, Tucon, AZ). Total body length, tibial length, femur length and growth plate depth were evaluated using NIH Image J software. Average depth, growth plate depth, and hypertrophic zone depths within growth plate were determined. 


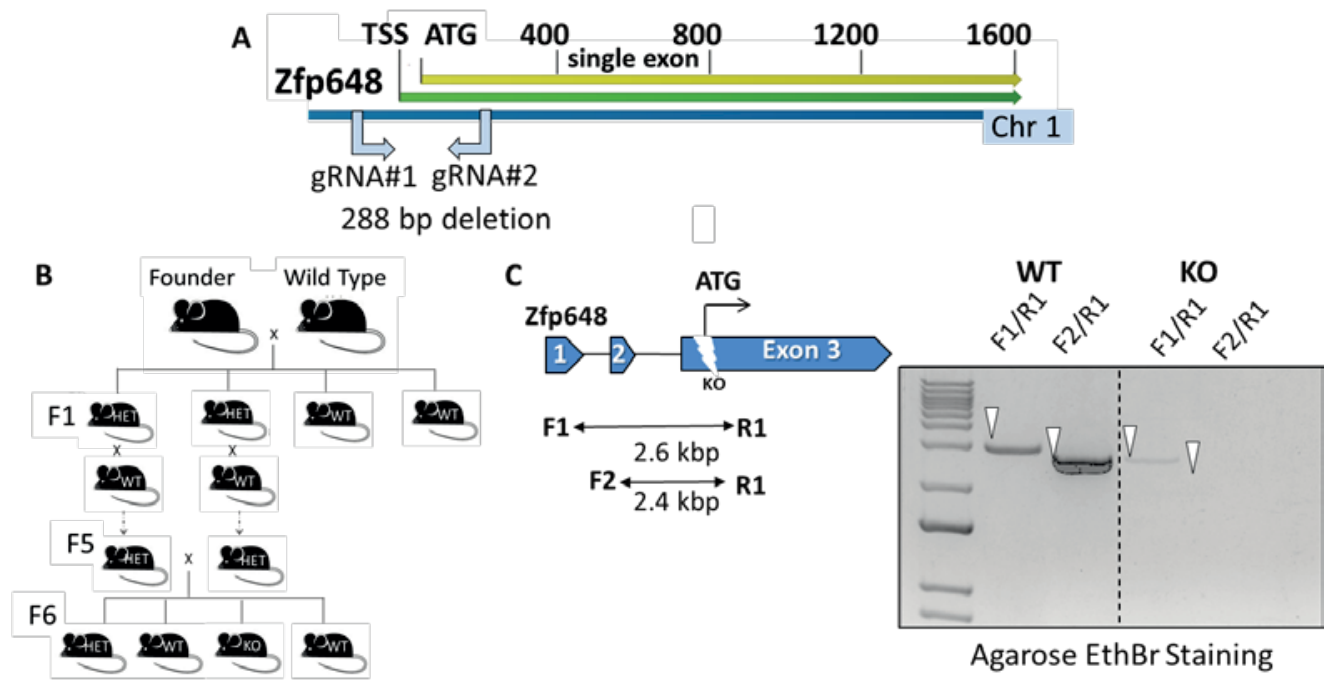

Figure 7.1. Schematic of the Zfp648 Crispr/Cas-9 strategy to generate the null mouse (A). Breeding strategy to eliminate off-target effects (B). Gene knockout confirmation using PCR analysis (C).

Comparison of epiphysis and growth plate depth was also compared among the different phenotypes. For histological analyses, mice were sacrificed at post-natal day 7. Right hind limb was fixed in $10 \%$ neutral buffered formalin, decalcified with 15\% EDTA and embedded in paraffin. Serial sections were taken at $5 \mu \mathrm{m}$ thickness and stained with Alcian Blue/Eosin or Safranin 0/Fast Green/Hematoxylin. Immunohistochemistry (IHC) was performed as previously described. Primary antibodies used included rabbit polyclonal anti-ZNF648 (1:500; 170269; Abcam), mouse monoclonal anti-COL2A1 (1:40; MAB8887; Millipore), rabbit monoclonal anti-COL1A1 (1:100; 138492; Abcam), mouse monoclonal anti-MKI67 (1:100; 238019; Abcam), rabbit monoclonal anti-mouse $\operatorname{IgG}(1: 100 ; 190475 ;$ Abcam) and goat anti-rabbit IgG (1:100; 205718; Abcam). Images were digitally photographed using the AxioVert A1 (Zeiss) and Zen software package (Zeiss).

\subsubsection{Statistical Analysis}

Data is presented as the mean \pm standard deviation (STD). Statistical analysis was performed using JMP® Pro v10.0.0 (SAS Institute), and $p$ values were 
determined using the Student T-test. Significance is noted in the figures when applicable as * if $p<0.05$ and $* *$ if $p<0.01$.

\subsection{RESULTS}

\subsubsection{Identification of ZNF648 as a novel cartilage specific zinc-finger protein}

Gene expression analysis performed on more than 1200 samples including bone, growth plate, and articular cartilage showed zinc finger 648 (ZNF648) was highly expressed and specific to cartilaginous tissues including growth plate, articular cartilage, chondrosarcoma and intervertebral disc compared to non-cartilaginous musculoskeletal tissues (Figure 7.2). Gene ontology analysis classified ZNF648 as a regulator of transcription and a nuclear factor.

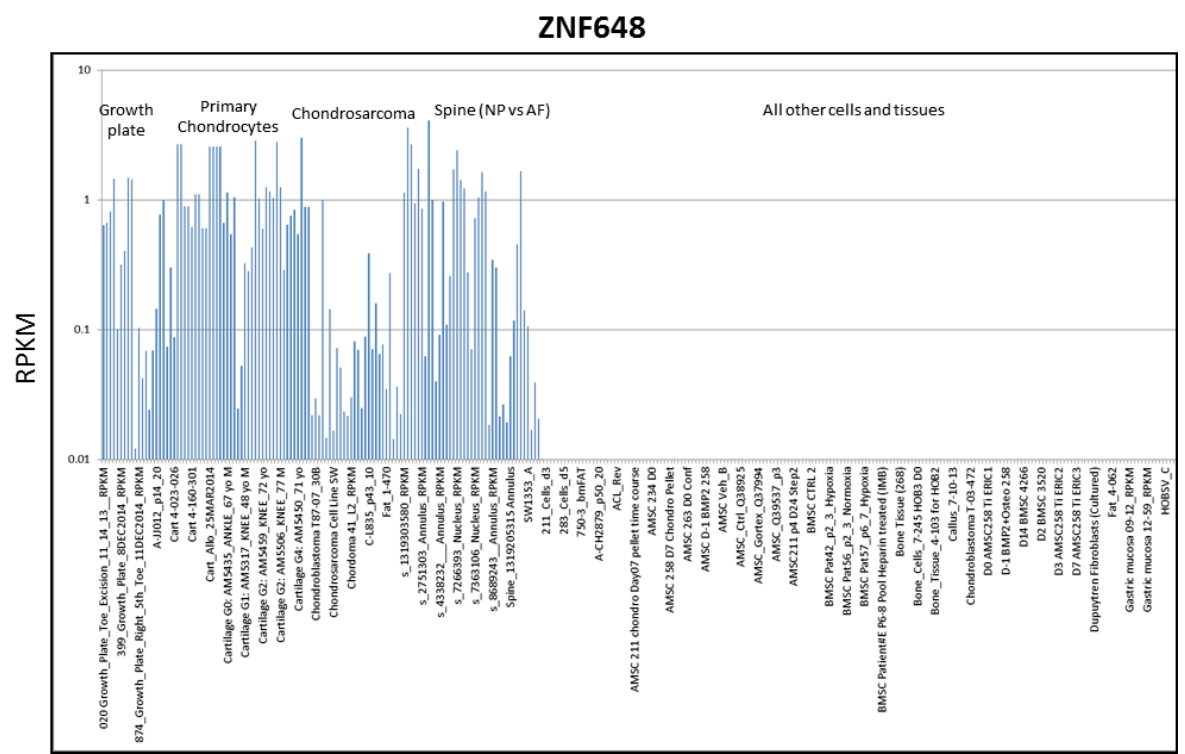

Figure 7.2 Identification of ZNF648 as a novel cartilage specific protein. ZNF648 expression in RNAseq is specific in cartilage when compared to other mesenchymal tissues and/or cells. All data is represented as RPKM. 


\subsubsection{ZNF648 and Zfp648 expression is highly specific to cartilage and not found in bone, following the expression pathways of COL2A1.}

Gene expression analysis on pediatric articular cartilage (AC), growth plate (GP), and bone was carried out using cartilage related markers collagen type II (COL2A1) and aggrecan (ACAN) as well as AKT1 and GAPDH as housekeeping genes (Figure 7.3). ZNF648 was highly expressed in pediatric articular cartilage, and expression correlated with that of both COL2A1 and ACAN (Figure 7.3A). Pediatric articular cartilage and growth plate had high expression of ZNF648, ACAN and COL2A1, whereas in primary human articular chondrocytes (hACs) obtained from adult patients, expression of ZNF648 was lost (Figure 7.3B). Proteoglycan-4 (PRG4) expression was positive only in articular cartilage tissue and cells. Human articular chondrocyte cells did express HIST2H4, a marker of proliferation, which was higher than in the native tissues. In addition, histological analysis revealed ZNF648 was expressed in both growth plate and chondrosarcoma tissues, but was not present in epiphyseal bone, comparable to COL2A1 (Figure 7.3C).

We next sought to determine if the cartilage specific expression of ZNF648 found in humans held true in mice. Wild type (WT) C57BL/6J mice were sacrificed at 1 week and 12 weeks of age, and qPCR gene expression analysis was performed on multiple tissues (Figure 7.4A and B). Zfp648 the mouse homolog of ZNF648 was highly specific for articular cartilage in mice and followed a similar expression pattern to Col2a1 and Acan. Interestingly, we observed that expression of Zfp648 and Col2a1 decreased in skeletally mature mice (12 weeks old) compared to younger animals ( 3 weeks old). Further comparison was performed between mouse immature articular chondrocytes (mACs) extracted from 1-week old mice and incubated in culture for 1 week, and ATDC5, a mouse immortalized chondrocyte cell line (Figure 7.4C). Robust expression of Zfp648 was found in immature mouse chondrocytes whereas the expression was low in ATDC5 immortalized cells. 

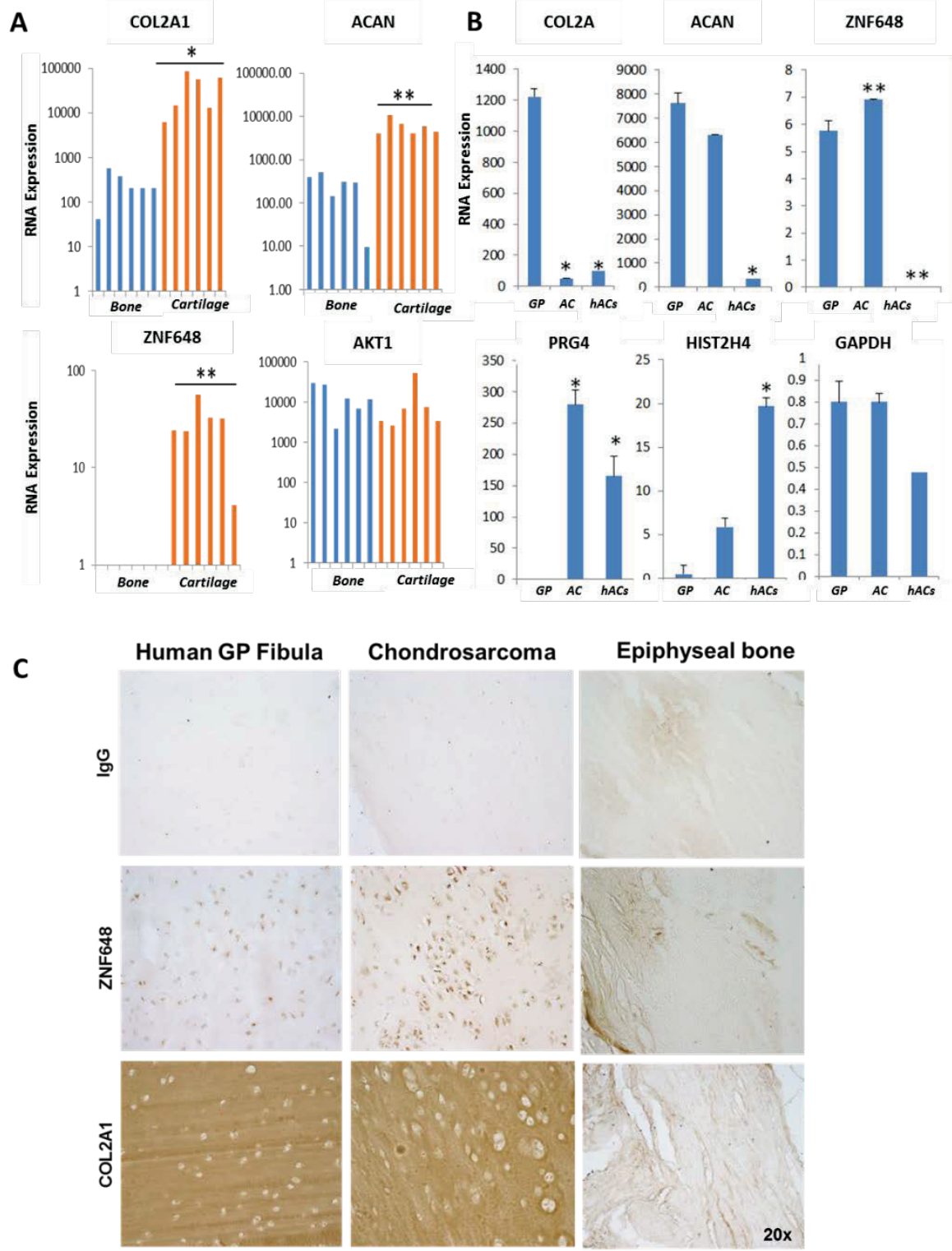

Figure 7.3 (A) Gene expression analysis of cartilage markers and ZNF648 on bone (blue bars) and cartilage (orange bars) tissues. (B) Gene expression analysis performed on pediatric articular cartilage (AC), growth plate (GP), and bone and compared to human articular chondrocytes (hACs). (C) Histological analysis of ZNF648 and COL2A1 on human GP fibula, chondrosarcoma tissue and epiphyseal bone. All data represented by mean \pm STDEV. Three biological replicates $(n=3)$ and three technical replicates were used. Statistical analysis was noted by * if $p$-value $<0.05$ and ** if $p$ value $<0.01$. 


\subsubsection{Expression of ZNF648 is abundant in primary chondrocytes}

whereas its expression decreases during cell culture and passaging ZNF648 expression in primary cultures of chondrocytes derived from growth plate and articular cartilage was analyzed during cell culture and passaging. We observed that while cells started proliferating more during passaging, they lost the expression of ZNF648 and chondrogenic marker COL2A1 (Figure 7.5A).

A

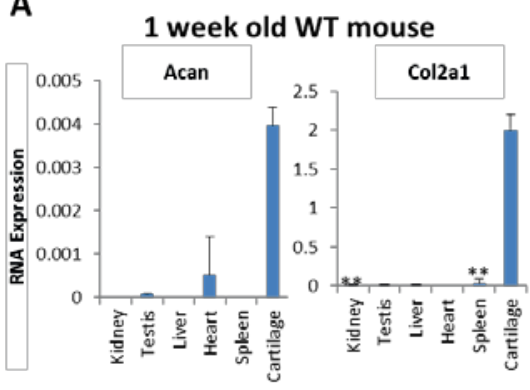

B
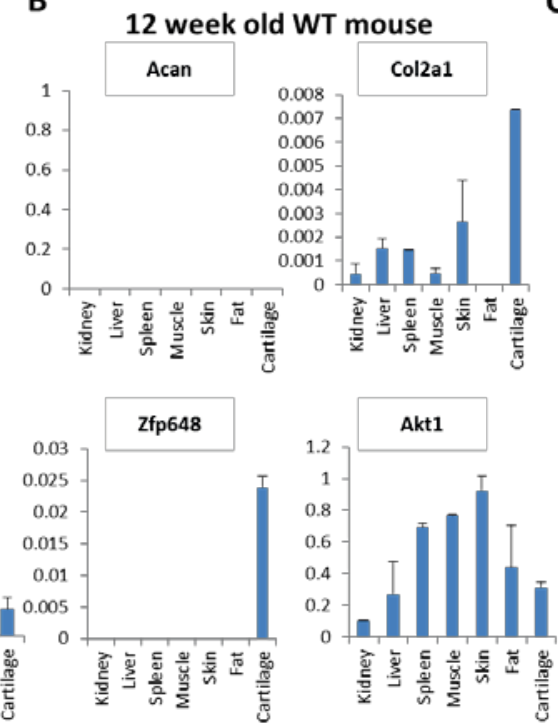

C
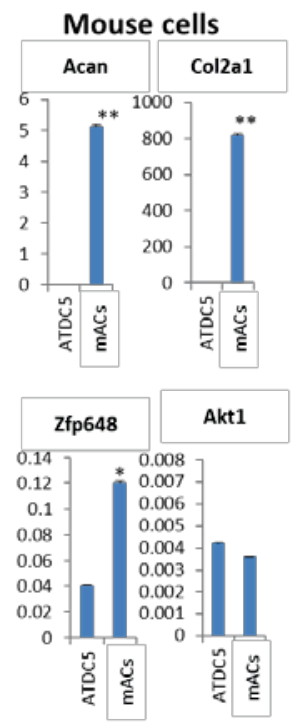

Figure 7.4 (A, B and C) Gene expression analysis of cartilage markers and Zfp648 was performed on mouse tissues, immortalized chondrocytes (ATDC5) and mouse immature articular chondrocytes (mACs). All data represented by mean \pm STDEV. Three biological replicates $(n=3)$ and three technical replicates were used. Statistical analysis was noted by * if $p$-value $<0.05$ and ** if $p$ value $<0.01$.

A comparison of allograft healthy cells (Figure 7.5 B), OA chondrocytes (Figure 7.5 $\mathrm{C}$ ) and growth plate chondrocytes (Figure 7.5 D) revealed that after 1 week in culture, the expression of cartilage markers COI2A1, ACAN and SOX9, and ZNF648 decreased in all cell cultures. In addition, ZNF648 mRNA expression was 10 times lower in $\mathrm{OA}$ chondrocytes than in healthy chondrocytes. In contrast, chondrocytes from articular cartilage seemed to proliferate more (e.g. HIST2H4 
expression increases) while a fibroblastic phenotype (e.g. COL1A1 expression increased) was induced. Growth plate chondrocytes did not proliferate in culture.
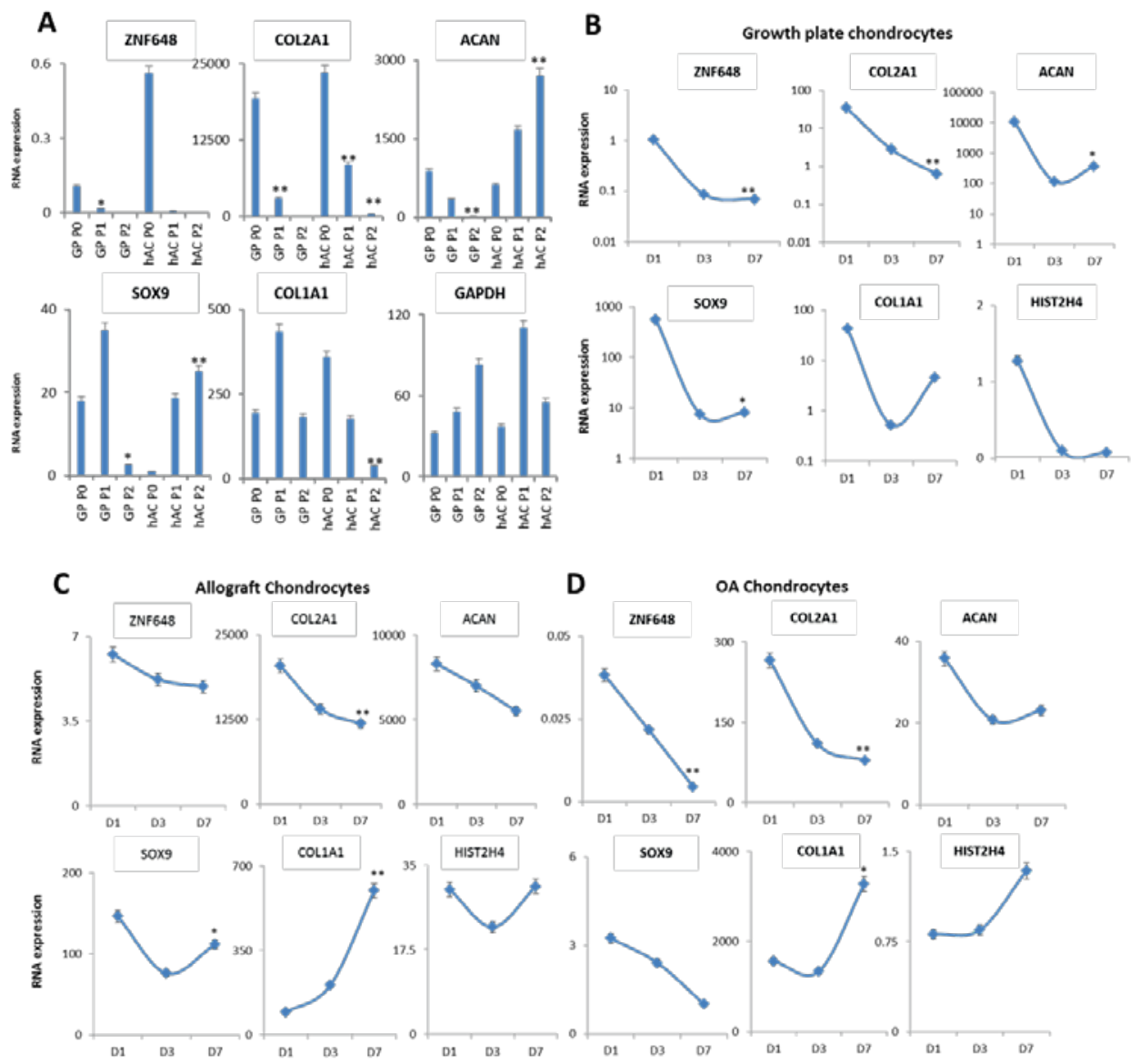

Figure 7.5 (A) ZNF648 expression decreases in passaged cells. (B, C and D) Growth plate and articular chondrocytes cultured for one week in high-density pellet. All data represented by mean \pm STDEV. Three biological replicates $(\mathrm{n}=3)$ and three technical replicates were used. Statistical analysis was noted by * if $p$-value $<0.05$ and $* *$ if $p$-value $<0.01$.

\subsubsection{ZNF648-mCherry plasmid overexpression in HEK293T cells induced $A C A N$ expression and cell proliferation.}

Overexpression experiments were performed by transfecting HEK293T cells using 2, 4 and $5 \mu$ g of an RFP-ZNF648 fusion construct (Origene) (Figure 7.6.A). An increased in nuclear localization of the fusion protein was seen while negative 
control showed whole cell staining (Figure 7.6.B). Protein lysates from the same experiment were analyzed. Lanes 1 to 3 showed no positive staining of ZNF648specific antibody in the negative control transfected cells. In contrast, ZNF648mCherry transfected cells (Lanes 4 to 6) exhibited significant ZNF648 positive staining (Figure 7.6C).

A

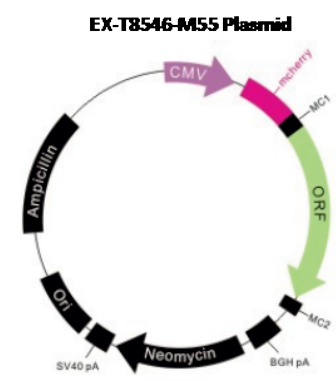

B
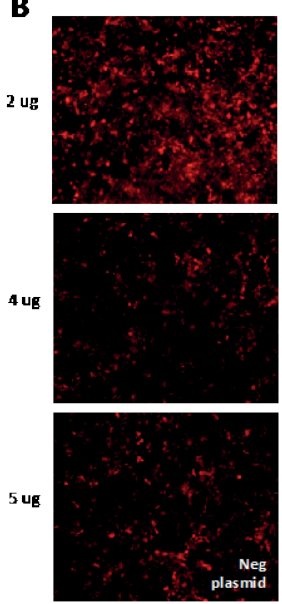
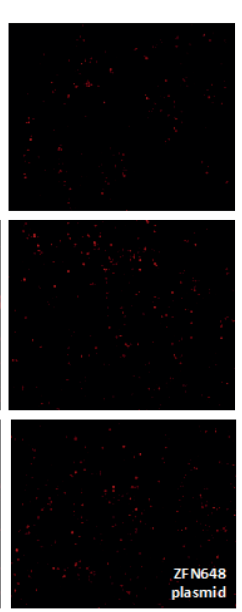

C

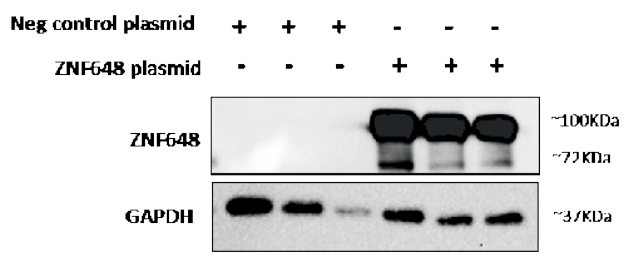

D
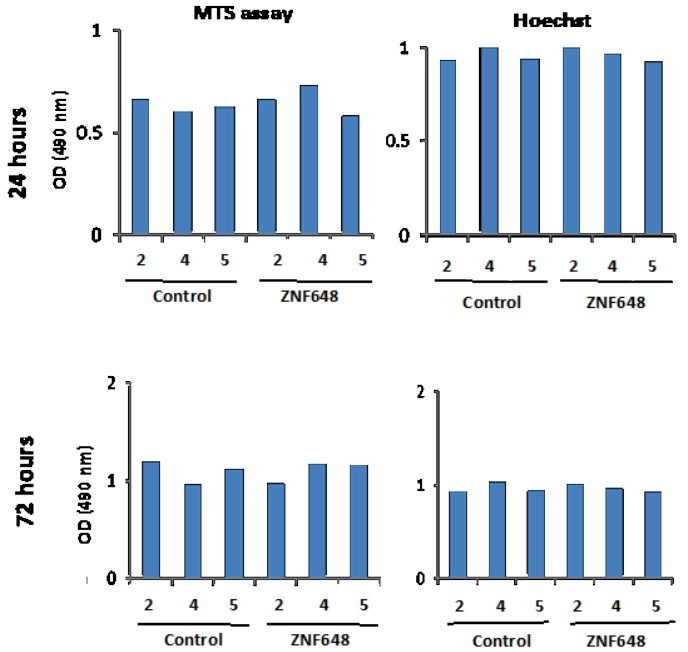

Figure 7.6 (A) ZNF648-mCherry plasmid vector used for overexpression experiments. (B)

Fluorescent microscopy from dose response experiments showing specific nuclear localization of cells transfected with the ZNF648-mCherry plasmid. (C) A ZNF648-mCherry fused protein was observed at $100 \mathrm{kDa}$ in western blotting analysis, and only in cells transfected with the plasmid. (D) MTS assay and Hoechst showed no toxicity from the transfection.

Two specific ZNF648 bands were observed in the blot with two different molecular weights, one band at 72kDa and another band at 100kDa. The ZNF648 protein had a predicted molecular weight of 72kDa. However, ZNF648-mCherry 
fusion protein had a predicted molecular weight of about $100 \mathrm{kDa}(72 \mathrm{kDa}$ of ZNF648 plus 37kDa of mCherry). The faint band observed at about $72 \mathrm{kDa}$ may represent some protein degradation.
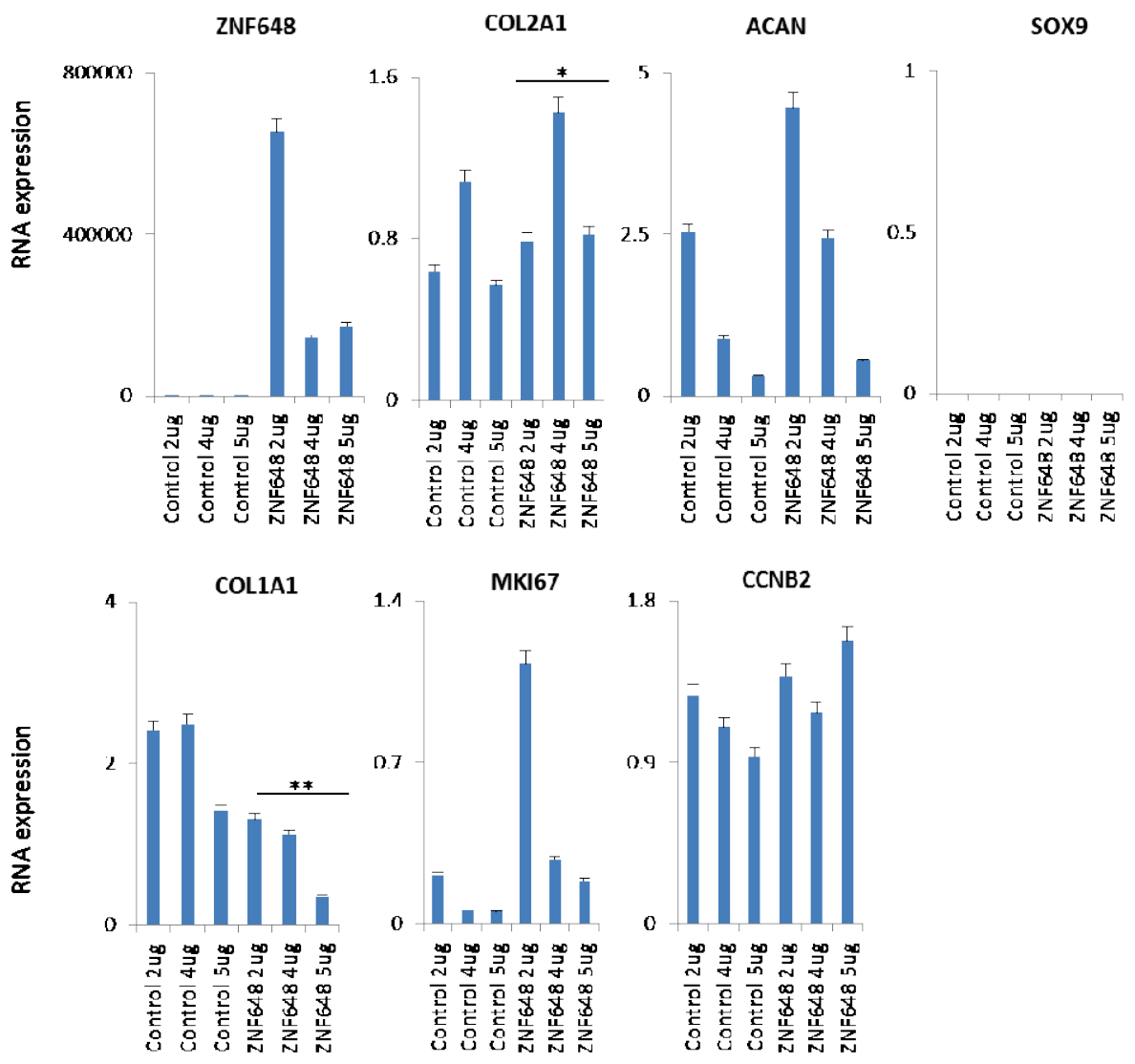

Figure 7.7 RT-qPCR showed up-regulation in mRNA expression of ZNF648 and ACAN while downregulating COL1A1 expression in transfected cells. Increased expression of MKI67 suggested an effect on proliferation of ZNF648 overexpression. The induction of COL2A1 and ACAN expression occurred independent of $S O X 9$, which was not induced after overexpression. All data represented by mean \pm STDEV. Statistical analysis (ZNF648 versus control) was noted by $*$ if $p$-value $<0.05$ and $* *$ if $p$-value $<0.01$.

GAPDH was observed at $37 \mathrm{kDa}$ and was used as a housekeeping control. Cell metabolic activity was evaluated at 24 hours and 72 hours after transfection. 
Cellular density was quantified using Hoechst staining. At both time points, no toxicity of ZNF648-mcherry overexpression was observed (Figure 7.6.D).

After 72 hours of transfection, a higher mRNA expression of ZNF648 was observed in cells transfected with the RFP-ZNF648 plasmid. COL2A1 expression remained steady, but increased significantly with the use of $4 \mu \mathrm{g}$ of RFP-ZNF648 plasmid when compared to the control (Figure 7.7). In addition, ACAN and MKI67 showed higher expression in cells transfected with RFP-ZNF648. In contrast, no expression of cartilage transcription factor SOX9 and a lower expression of COL1A1 were observed in cells overexpressing ZNF648. No changes in proliferation of the cells were suggested by the steady expression of CCNB2 in both conditions. Thus, overexpression of ZNF648 was sufficient to induce the upregulation of ACAN expression irrespective of SOX9 expression whilst decreasing the expression of COL1A1, suggesting a down-regulation of a fibroblastic signaling in the cells.

\subsubsection{ZNF648 overexpression regulates specific genes in human articular cartilage and growth plate chondrocytes.}

To further study the subcellular localization of ZNF648, overexpression experiments were performed by transfecting HEK293T cells for 72 hours using $5 \mu \mathrm{g}$ of ZNF648 tagged with eGFP (Origene) (Figure 7.8.A). ZNF648 and housekeeping gene (GAPDH) expression were compared side by side in nuclear extracts, cytoplasmic extracts, and whole cell lysates (Figure 7.8.B). Nontransfected cells (Lane 1) and negative control plasmid transfected cells (Lanes 2 to 4) did not stain with a ZNF648 specific antibody. Cells transfected with ZNF648-eGFP showed positive staining in whole cell lysates and nuclear extracts, whereas no staining was observed in the cytoplasmic extracts. Thus, our data demonstrated that chondrocyte specific transcription factor ZNF648 is a 72-kDa protein that localizes in the nucleus. GAPDH was observed at $37 \mathrm{kDa}$ and was used as a housekeeping control. 
Next, we explored the effect of overexpression of ZNF648 in human primary chondrocytes from articular cartilage and growth plate. Both cells were obtained from healthy pediatric tissue and were from the same donor. In untransfected cells, ZNF648 positive staining was localized in both nucleus and cytoplasm. This was likely due to the endogenous levels of ZNF648 in human primary chondrocytes. Forced overexpression of ZNF648 plasmid reduced COL2A1 expression (green, Figure 7.8C) in articular chondrocytes compared to the negative control, whereas forced overexpression in growth plate chondrocytes increased COL2A1 staining (green, Figure 7.8D) compared to the negative control.
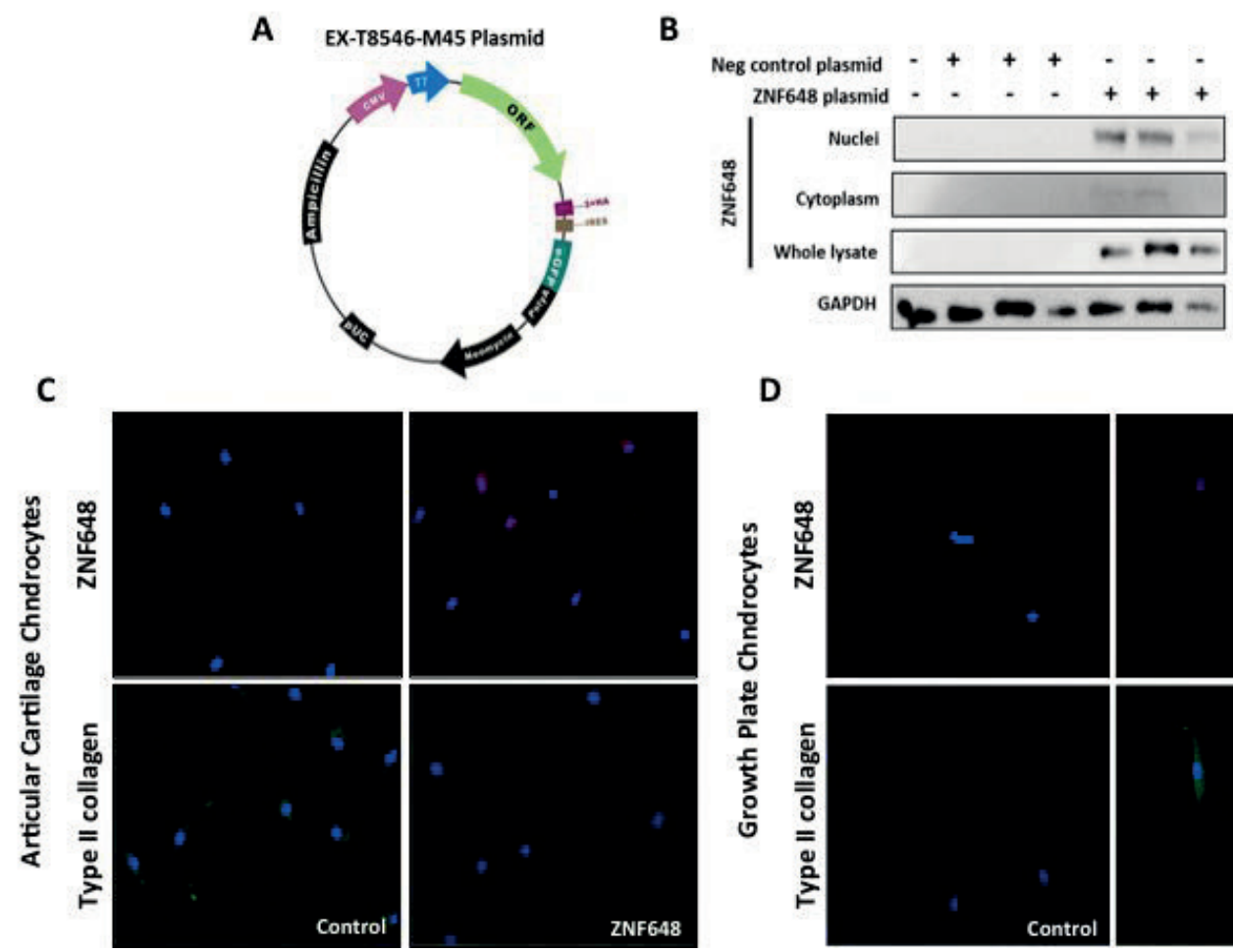

D

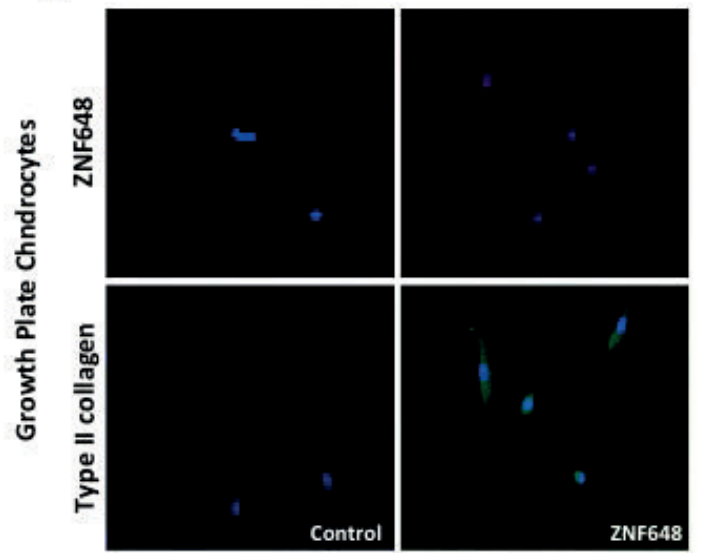

Figure 7.8 (A) Plasmid vector containing ZNF648 tagged with eGFP used for overexpression experiments. (B) Nuclear localization of ZNF648 protein in HEK293T cells overexpressing ZNF648. (C and D) Overexpression experiments in human articular cartilage and growth plate chondrocytes showing decreased expression of collagen type II in articular chondrocytes and increased expression in growth plate chondrocytes. 


\subsubsection{Knockdown experiments in human primary chondrocytes imply ZNF648 regulates COL2A1 expression.}

We performed further work to understand the cellular effects resulting from an absence of ZNF648. In articular chondrocytes, knockdown of ZNF648 was observed and resulted in loss of COL2A1 and MKI67 expression. This suggested that ZNF648 positively regulates expression of key cartilage genes and cell cycle and/or proliferation of the cells (Figure 7.9.A).
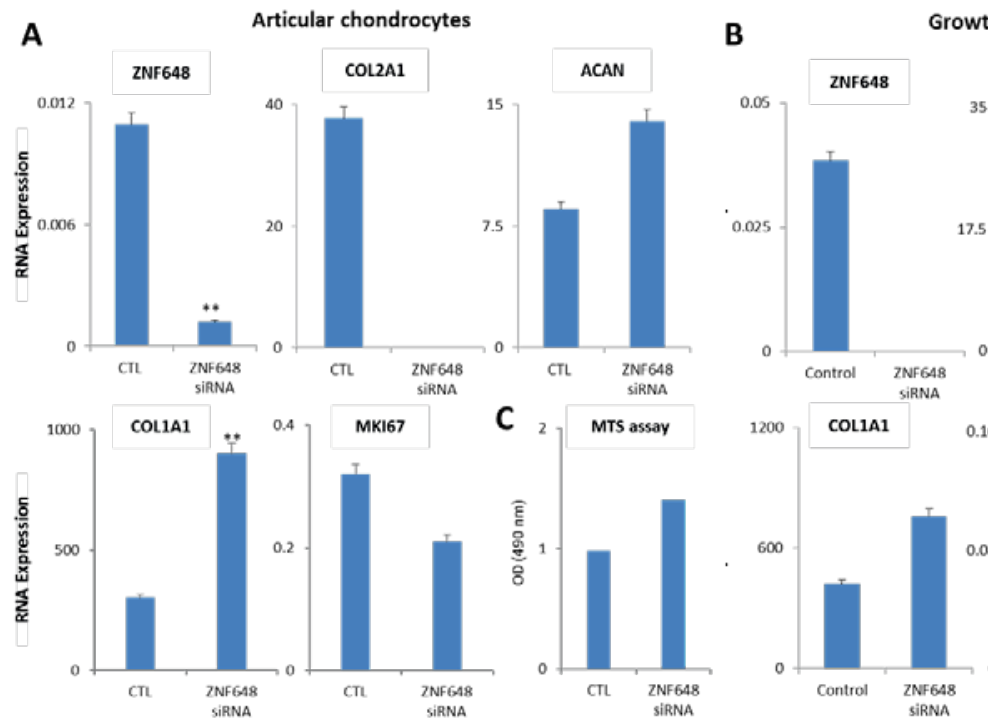

Growth Plate chondrocytes
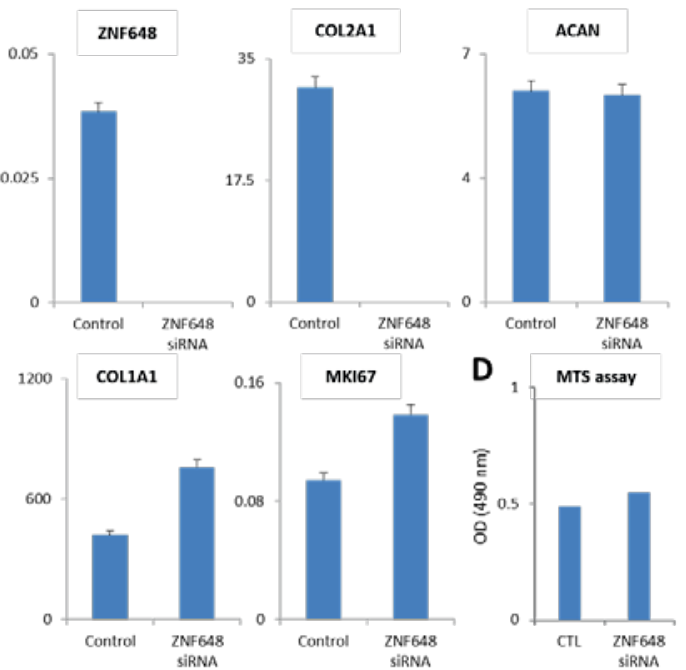

Figure 7.9 (A) Articular chondrocytes were transfected with a ZNF648 siRNA. ZNF648 loss was followed by decrease of COL2A1 and proliferation marker MKI67. In contrast, COL1A1 and ACAN were up regulated. (B) ZNF648 siRNA transfection in growth plate chondrocytes obtained from human pediatric patients was performed. ZNF648 loss was followed by a decrease in COL2A1 expression, whereas COL1A1 and MKI67 expression were increased. (C and D) No toxicity was observed from the transfection as assessed using an MTS assay. All data represented by mean \pm STDEV. Statistical analysis was noted by * if $p$-value $<0.05$ and $* *$ if $p$-value $<0.01$.

In contrast, an increased expression of ACAN and COL1A1 was observed in the absence of ZNF648. Similar results were found for growth plate chondrocytes (Figure 7.9.B). Knockdown of ZNF648 decreased COL2A1 expression while increasing COL1A1. However, in growth plate chondrocytes the absence of ZNF648 increased the expression of proliferation marker MKI67 while ACAN 
expression was steady. Hence, ZNF648 correlated with COL2A1, but not ACAN and MKI67 expression in either chondrocyte cell type. No toxicity was observed from the transfection as assessed using an MTS assay. (Figure 7.9 C and D).

\subsubsection{ZNF648 controls COL2A1 induction in human immortalized TC28A2 cells and chondrosarcoma cell line $\mathbf{L} 835$.}

ZNF648 knock down experiments were also performed in human immortalized TC28A2 cells and human L835 chondrosarcoma cell line. Due to the absence of ZNF648 in TC28A2, overexpression experiments were conducted.

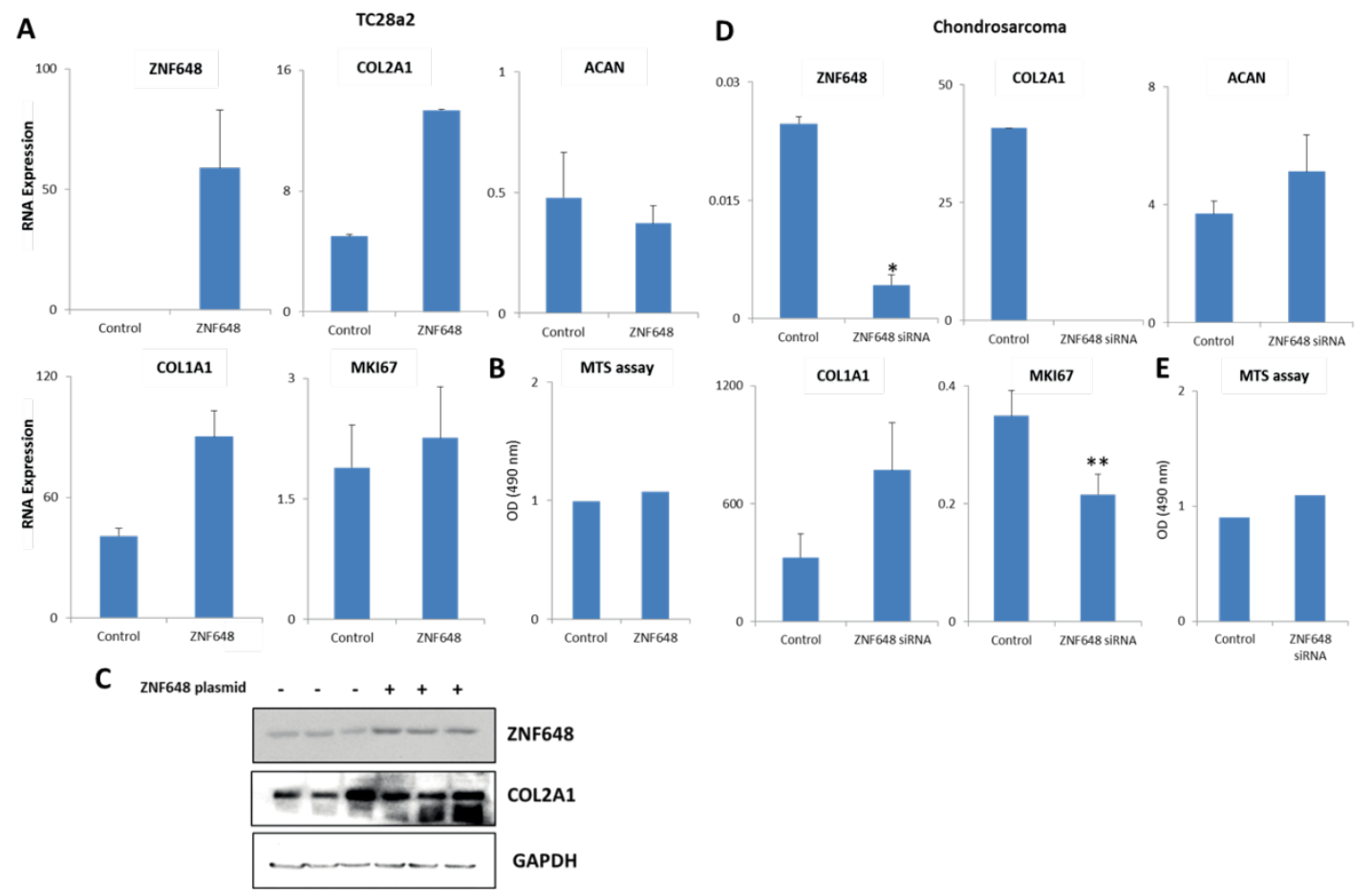

Figure 7.10 (A) TC28A2 human immortalized chondrocytes transfected with a ZNF648 expression vector showed increased expression of COL1A1, COL2A1and MKI67 expression. (B and E) Metabolic activity assay did not show any signs of toxicity on the cells. (C) Increased protein expression of specific ZNF648 antibody was shown in overexpressed TC28A2 cells. (D) Human chondrosarcoma

cell line L835 transfected with ZNF648 siRNA decreased expression of ZNF648, followed by a decreased in COL2A1and MKI67. However, COL1A1 and ACAN expression increased in ZNF648 depleted cells. All data represented by mean \pm STDEV. Statistical analysis was noted by * if $p$-value

$$
<0.05 \text { and }^{* *} \text { if } p \text {-value }<0.01 \text {. }
$$


RNA was collected and extracted after 72 hours of transfection. TC28A2 cells showed overexpression of ZNF648 and simultaneously increased COL2A1, COL1A1 and MKI67 expression (Figure 7.10 A). However, ACAN expression decreased with ZNF648 overexpression. Protein analysis depicts an increase in ZNF648 expression (Lanes 4 to 6) in TC28A2 cells overexpressing ZNF648. Lower expression was detected in non-transfected cells. Collagen type II expression followed the trend of ZNF648 (Figure 7.10 C). GAPDH was used as a sample loading control. In addition, knock down experiments in chondrosarcoma cell line L835, showed a decreased of ZNF648 gene expression followed by downregulation of COL2A1 and MKI67. In contrast, ACAN and COL1A1 expression increased in the absence of ZNF648 (Figure 7.10.D). MTS assays showed no toxic effects of the transfection on the cells (Figure 7.10 B and $\boldsymbol{E}$ ). Immortalized cells showed a similar gene expression trend compared to human primary cells, implying a positive association between ZNF648 and COL2A1 expression, and a negative association with COL1A1 expression. Consequently, ZNF648 seems to regulate COL1A1 and COL2A1 expression in both, primary cells and in immortalized tumor cells.

\subsubsection{ZNF648 mouse homolog Zfp648 regulates Col2a1 induction in ATDC5 and in immature mouse articular chondrocytes.}

Transfection experiments were performed on immortalized mouse chondrocytes and mouse articular chondrocytes. Due to the absence of Zfp648 in ATDC5, overexpression experiments were conducted instead. RNA expression analysis conducted using RT-qPCR showed overexpression of Zfp648 in ATDC5 increased Col2a1 whilst decreasing expression of Col1a1 and Mki67 (Figure 7.11 A). Western blot analysis showed Zfp648 was increased in transfected cells (Lanes 3 to 6) compared to control (Lanes 1 to 3 ). We did not detect Col2a1 expression in ATDC5 (Figure 7.11 C). 

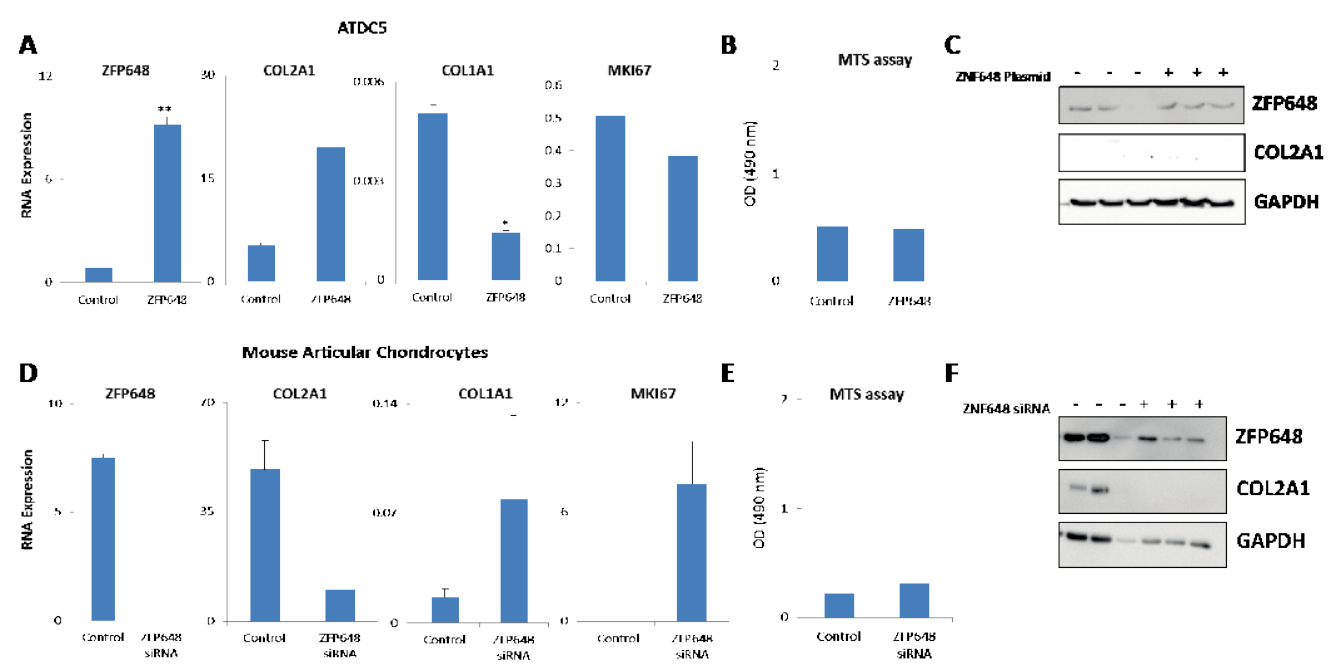

Figure 7.11 (A) ATDC5 mouse immortalized chondrocytes transfected with a Zfp648 expression vector showed increased expression of Col2a1 while decreasing Col1a1 and Mki67. (B and E) No signs of toxicity on the cells were observed. (C) Increased ZFP648 protein expression was shown in overexpressed cells only. (D) Human chondrosarcoma cell line L835 transfected with ZNF648 siRNA decreased expression of Zfp648, which was associated with a decrease in Col2a1 and Mki67. In contrast, Co1a1 and Acan expression increased in Zfp648 depleted cells. (F) Depletion of Zfp648 and Col2a1 was observed in Zfp648 siRNA transfected cells. All data represented by mean \pm STDEV.

Statistical analysis was noted by $*$ if $p$-value $<0.05$ and ${ }^{* *}$ if $p$-value $<0.01$.

Likewise, Zfp648 knockdown in mouse articular chondrocytes decreased Zfp648 mRNA expression and down-regulated Col2a1. In contrast, Col1a1 and Mki67 were increased in the absence of Zfp648 (Figure 7.11 D). Protein analysis showed depletion of Zfp648 and collagen type II expression in Zfp648 siRNA transfected cells (Lanes 3 to 6) compared to control (Lanes 1 to 3) (Figure 7.11 F). No differences in MTS metabolic activity were observed (Figure 7.11 B and $\boldsymbol{E}$ ). Thus, both immortalized and mouse primary cells mouse followed the trend previously observed in human TC28A2 and chondrosarcoma cells. Zfp648 overexpression induced Col2a1 expression whereas inhibition decreased Col2a1 expression. An up-regulation of Mki67 proliferation marker may be an indicative for increased proliferation. 


\subsubsection{Zfp648 null mice survive beyond birth and do not have overt skeletal defects.}

The effect of Zfp648 ablation in mice was determined. Homozygous Zfp648 null mice survived beyond birth. No overt skeletal defects were observed after 12 weeks (Figure 7.12 A and B). Weight was obtained at three different time points, no difference among the phenotypes was observed (Figure 7.12 C). Zfp648 expression was increased over time in wild type (WT) and was depleted in cartilage of null mice (KO) (Figure 7.12 D). In mouse cartilage native tissue an antagonistic effect for Col2a1 and Col1a1 was observed.

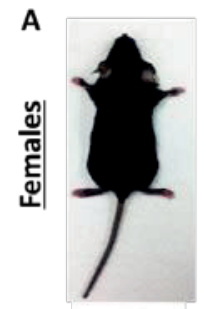

WT

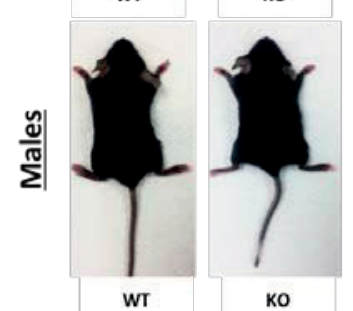

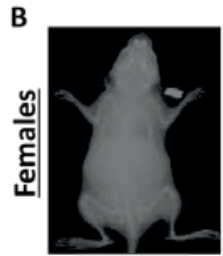

WT

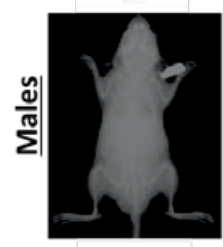

WT

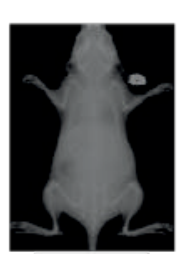

ko

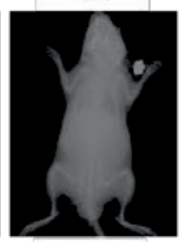

KO

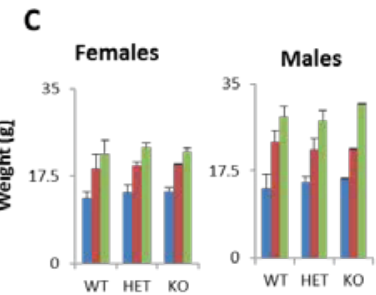

D

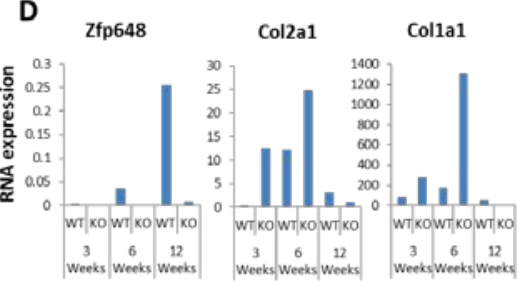

Figure 7.12 (A and B) Skeletal defects were not observed in null mice. (C) Null mice, heterozygous and wild types were weighted after 3, 6 and 12 weeks. No differences in weight among the phenotypes were observed. (D) RNA expression of Zfp648 shows the depletion in null mice. In mouse cartilage native tissue an antagonistic effect for Col2a1 and Col1a1 was observed. Data represented by mean \pm STDEV. Statistical analysis was noted by * if $p$-value $<0.05$ and ** if $p$-value $<0.01$.

7.3.10 Young Zfp648 null mouse exhibit reduced skeletal size and growth plate width with a delayed secondary ossification center and disorganization of growth plate zones.

To assess the skeletal phenotypic differences of Zfp648 null mice at earlier time points, 2-week-old null and wild type mice were sacrificed. In Figure 7.13.A we 
observed that RNA expression of Zfp648 was lost in null mice. A reduced expression of Col2a1 and Acan was also observed. Radiographic analysis on both phenotypes revealed a reduced skeletal size (whole body, femur and tibial length) in Zfp648 null mice when compared to wild type mice. In contrast, a higher growth plate width was observed in Zfp648 null mice (Figure 7.13.B).
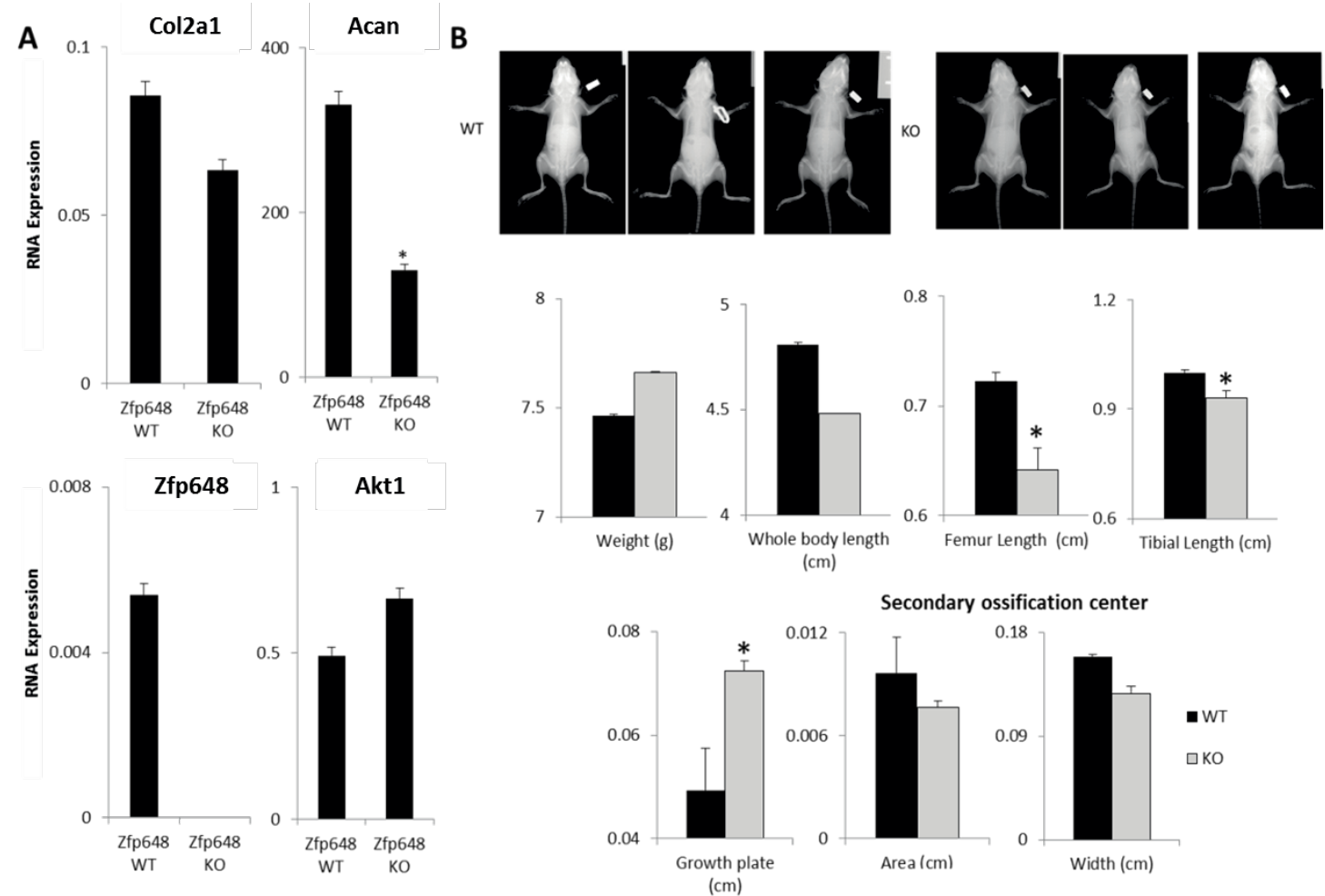

Figure 7.13 (A) RNA expression shows decreased of Zfp648, Col2a1 and Acan in 2-week old Zfp648 null mice. (B) Radiographic analysis showed a reduced skeletal size shown by reduced in whole body, femur and tibial length. In addition, growth plate height was significantly increased in null mice. Secondary ossification center measured in the radiographies showed a decrease in area and width in null mice. All data represented by mean \pm STDEV. Statistical analysis was noted by * if

$$
p \text {-value }<0.05 \text { and } * * \text { if } p \text {-value }<0.01 \text {. }
$$

Secondary ossification center area and width, were measured from the radiographies, and showed a decreased in null mice compared to wild type. 
Histological analysis performed on mice tibias of 2-week-old pups using Safranin $0 /$ Fast green/Hematoxylin staining revealed a delay in the onset of secondary ossification center formation in Zfp648 null mice (Figure 7.14.A). A lack of mineralized tissue in the location of the secondary ossification center was also observed by Alcian blue/Eosin staining. A cartilage specific staining was observed all over the growth plate zone in null mice, whereas growth plate and articular cartilage were well defined in wild type mice.

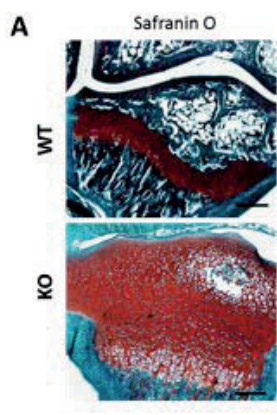

C

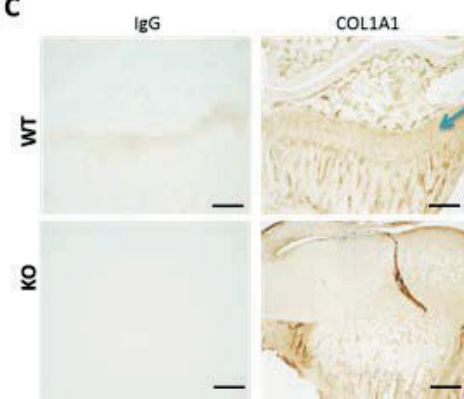

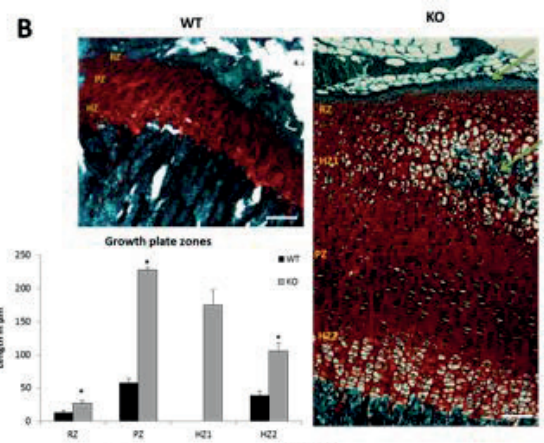

COL2A1 MKI67
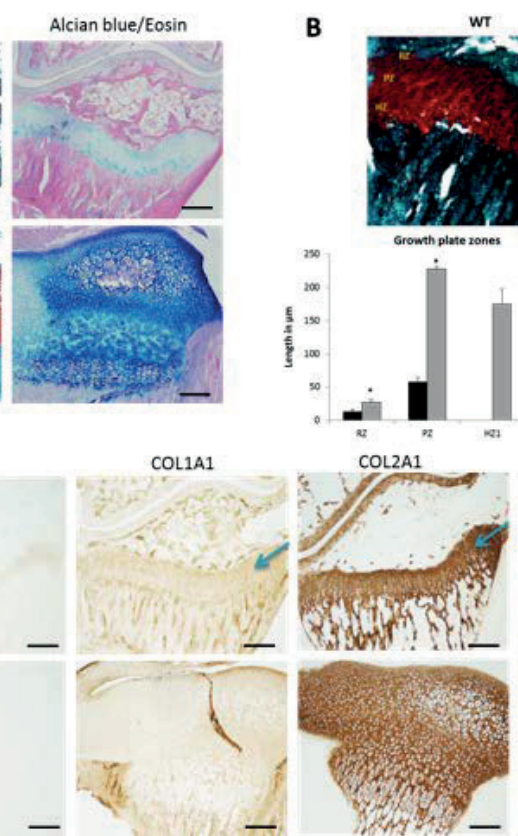

Figure 7.14. (A) Safranin 0/Fast green/ Hematoxylin staining and alcian blue/eosin showed differences between wild type and null mice at 2 weeks of age. Scale bars (black bars) indicate $100 \mu \mathrm{m}$. (B) Disorganization of growth plate zones and differences in their lengths were observed and measured. Scale bars (white bars) indicate $200 \mu \mathrm{m}$. Measurements were represented by mean \pm STDEV. Statistical analysis was noted by * if $p$-value $<0.05$ and $* *$ if $p$-value $<0.01$.

(C)Immunohistochemistry revealed specific expression of cartilage marker Col2a1 overall growth plate zones in null mice. In contrast, Col1a1 expression was absent. Mki67 expression depicted an increase of proliferation in all zones. Scale bars (black bars) indicate $100 \mu \mathrm{m}$. 
In Figure 7.14.B we observed the difference in growth plate zonal distribution in null mice. There was no articular cartilage defined and low mineralization was observed in the zone of the secondary ossification center. Two hypertrophic chondrocyte zones were observed in the null mice and were measured. Measurements exposed the increase in length of all growth plate zones in Zfp648 null mice. Immunohistochemistry using specific antibodies for Col2a1, Col1a1 and Mki67 was performed to assess differences in cartilage specific staining (Figure 7.14.C). Wild type mice showed specific staining of Col2a1 in the growth plate and articular cartilage; whereas surrounding mineralized tissue was specifically stained with Col1a1. Mki67 was not expressed in the WT mice. Null mice showed an overall staining of growth plate with Col2a1 and no staining with Col1a1. Faint Col1a1 staining was found at the upper part of the tibia, where the articular cartilage should be located. In addition, Mki67 specific staining was found along the growth plate showing the overall increase in proliferation in the growth plate zone on this phenotype.

\subsection{DISCUSSION}

The clinical need to develop new cartilage repair strategies arises from the fact that cartilage has a low self-repair capacity and is not suitable to respond to traumatic injury. Therefore, these injuries often result in altered biomechanics of the joint and joint instability. The mechanism by which articular cartilage homeostasis is maintained and is able to resist hypertrophic differentiation and subsequent endochondral ossification remains unknown. Thus, the complex molecular events resulting in the induction and maintenance of the chondrogenic phenotype during embryogenesis, may lead to the identification of novel mechanisms involved in cartilage formation. Fundamental knowledge of these mechanisms might prove transcription factors such as Zinc Finger 648 (ZNF648) protein to be crucial for cartilage repair strategies. 
RNA-seq analysis revealed a unique expression of ZNF648 in all cartilaginous tissues. Primary growth plate cells showed a higher expression of this transcription factor when compared to primary articular chondrocytes. Fagerberg L et al. previously published an RNAseq analysis performed on normal tissues derived from 95 human individuals representing 27 different tissues but lacking cartilage tissue [17]. They found ZNF648 expression in testis and ovaries at substantially lower values of 0.100 and 0.125 RPKM, respectively, compared to values ranging between 0.5 and 3 RPKM in our data set. In liver and skin, even lower levels of ZNF648 were found. We therefore concluded that ZNF648 is highly specific for cartilaginous tissues with minor expression in reproductive organs.

ZNF648 belongs to the transcription factor family C2H2-ZNF that is found only in eukaryotic species. Zinc finger proteins (ZNF) are mostly expressed in human and mouse. It has been suggested that these can act alone or partner with other transcription factors (TFs) to create distinct functionalities [18]. These TFs constitute the single largest family of regulators in the human genome $(\sim 400$ genes). A large part of these TFs have a tissue-specific expression, suggesting that they control processes that are characteristic of individual tissues. For example ZNF337 is highly expressed in fetal brain, which suggests an involvement in brain development. However, the biological functions of most of these TFs are unknown [19]. In our study, this ZNF648 protein seems to maintain normal differentiation of pre-committed chondrogenic mesenchymal progenitor cells into chondrocytes in the growth plate, whereas in later stages of cartilage maturation ZNF648 expression is reduced. The highly expression of ZNF648 in growth plate suggests an involvement of in cartilage formation.

Although the expression of ZNF648 is present in primary tissues, it is significantly reduced when cells are removed from the native tissue environment. The depletion of ZNF648 expression was always associated with a 
reduction in COL2A1 expression. In the case of articular chondrocytes it was interesting to observe that throughout the passaging of these cells, SOX9 and ACAN expression were enhanced. This phenomenon was not observed in cells cultured in vitro. Interestingly, an enhanced expression of ZNF648 increased collagen type II expression while decreasing collagen type I. This concept would be ideal for formation of hyaline cartilage over fibrocartilage in tissue regeneration strategies. Still, it is unclear if COL2A1 is a direct target of ZNF648. Further study will help elucidate ZNF648 signaling mechanisms for cartilage development. Previously a list of target genes of transcription factors predicted using known transcription factor binding sites by computational association, showed SOX2, SOX5, ARNT (HIF1B), RUNX2, SMAD2/3, HIF1A, POU5F1, HAPLN1, GLI1, GLI2, GLI3 and SOX9, among the predicted targets of transcription factors ZNF648 [22-25]. In addition, among the predicted pathways of ZNF648 they found glycosaminoglycan degradation and biosynthesis, cell cycle, fatty acid biosynthesis, WNT signaling, Hedhegod signaling and signaling pathways regulating pluripotency of stem cells [23]. The differential gene expression observed in our study between growth plate and articular chondrocytes could indicate a regulatory mechanism of ZNF648 to promote chondrogenesis and cartilage formation; this could occur by regulation of other cartilage specific genes and not necessarily of COL2A1, but by interacting with one or various pathways involved in cartilage development, including SOX9 and RUNX2.

Some of the ZNF proteins act also as repressor, depending of the domain on the N-terminal site (e.g ZNF133 and ZNF 140) [20], whereas they are also involved in cell differentiation, cell proliferation, apoptosis, and neoplastic transformation [21]. In addition, a couple of ZNF proteins have been previously linked with chondrogenesis. ZNF219 and ZNF449 were both described as implicated in SOX9 mediated chondrogenesis $[30,31]$. Whole mount in situ hybridization experiments indicated that Zfp219 was specifically expressed in developing 
limbs where Col2a1 and Sox9 were strongly expressed. Zfp219 markedly enhanced the transcriptional activity of Sox 9 on the Col2a1 gene promoter, suggesting Zfp219 as important in the regulation of chondrocyte differentiation as a transcriptional partner of Sox9 [30]. Also, ZNF proteins are thought to integrate numerous signals through the action of microRNAs. Target genes of microRNAs were predicted by searching genes for sites matching conserved and noncoserved miRNA seed regions. They predicted miR-210 as one of the miRNAS involved with ZNF648 [26- 28]. In Chapters 4 and 5, we exhibited miR-210 as essential for chondrogenesis of stem cells. The interaction of miR-210 and ZNF648 could indicate a regulatory mechanism to induced or repress the expression of COL2A1 and ACAN.

Previously, predicted mouse and human phenotype were described in the literature [22-25]. Cartilage related phenotypes were associated with ZNF648 loss of expression. Among them, abnormal nose, ear, trachea and larynx morphology, abnormal embryonic tissue, abnormal embryogenesis phenotype were associated in mouse; whilst polydactyly, abnormal bone, short limb and short stature, and abnormal ear and nose morphology were linked to the human phenotype. Remarkably, the phenotype obtained from 2-week-old null mouse indicates an important role of this transcription factor in the development of long bones and during endochondral ossification stages. A mineralized cartilage instead of bone was observed between articular cartilage and growth plate. Furthermore, overexpression of ZNF648 in cells induces up-regulation of MKI67 that was also observed in Zfp648 null mice. These observations may implicate ZNF648 in the regulation of chondrocyte proliferation.

In this study we aimed to analyze driving molecular factors such as transcription factor ZNF648 that may directly regulate cartilage development or homeostasis through modulating gene expression and other cartilage-related signaling pathways. Thus, development of novel approaches to improve cartilage 
regeneration from a mechanistic and molecular point using ZNF648 to maintain cartilage homeostasis is thought to play and essential role in future clinical therapies. 


\section{REFERENCES}

1. Al Kaissi A, Nabil NM, Kenis V, Melchenko E, Morenko E, Grill F, Ganger R, Kircher SG.: Schmid's Type of Metaphyseal Chondrodysplasia: Diagnosis and Management. Orthop Surg 2018:241-246.

2. Aponte-Tinao LA, Ayerza MA, Muscolo DL, Ing FM, Farfalli GL.: What Are the Complications of Allograft Reconstructions for Sarcoma Resection in Children Younger Than 10 Years at Long-term Followup? Clin Orthop Relat Res 2018:548-555.

3. Sabharwal S, Kumar A: Methods for Assessing Leg Length Discrepancy. Clin Orthop Relat Res 2008:2910-2922.

4. Sabharwal S, Sabharwal S: Growth Plate Injuries of the Lower Extremity: Case Examples and Lessons Learned. Indian J Orthop 2018:462-469.

5. Miyamura S, Oka K, Shigi A, Abe S, Yoshikawa H, Murase T: Physeal bar resection using a patient-specific guide with intramedullary endoscopic assistance for partial physeal arrest of the distal radius. Arch Orthop Trauma Surg 2018:1179-1188.

6. Hasler CC, Krieg AH: Current concepts of leg lengthening. J Child Orthop 2012:89-104.

7. Bolbasov EN, Kononovich NA, Gorbach EN, Khlusov IA, Golovkin AS, Stankevich KS, Ignatov VP, Bouznik VM, Anissimov YG, Tverdokhlebov SI, Popkov AV.: Flexible intramedullary nails for limb lengthening: a comprehensive comparative study of three nails types. Biomed Mater 2019:14(12):025005.

8. Stevens PM: The role of guided growth as it relates to limb lengthening. J Child Orthop 2016:479-486.

9. Lewallen EA, Bonin C, Li X, Smith J, Karperien M, Larson AN, Lewallen DG, Cool SM, Westendorf JJ, Krych AJ, Leontovich AA, Im HJ, van Wijnen AJ: The synovial microenvironment of osteoarthritic joints alters RNA-seq expression profiles of human primary articular chondrocytes. Gene 2016:456-464.

10. Dudakovic A, Camilleri E, Riester SM, Lewallen EA, Kvasha S, Chen X, Radel DJ, Anderson JM, Nair AA, Evans JM, et al: High-resolution molecular validation of selfrenewal and spontaneous differentiation in clinical-grade adipose-tissue derived human mesenchymal stem cells. J Cell Biochem 2014, 115:1816-1828.

11. Kim D, Trapnell C, Pimentel H, Kelley R, Salzberg SL.: TopHat2: accurate alignment of transcriptomes in the presence of insertions, deletions and gene fusions. Genome biology 2013:14:R36.

12. Anders PT: HTSeq-a Python framework to work with high-throughput sequencing data. Bioinformatics 2015:166-169.

13. Galeano-Garces C, Camilleri E, Riester SM, Dudakovic A, Larson DR, Qu W, Smith J, Dietz AB, Im HJ, Krych AJ, Larson AN, Karperien M, van Wijnen AJ.: Molecular Validation of Chondrogenic Differentiation and Hypoxia Responsiveness of Platelet-Lysate Expanded Adipose Tissue-Derived Human Mesenchymal Stromal Cells. Cartilage 2017:283-299.

14. Camilleri ET, Dudakovic A, Riester SM, Galeano-Garces C, Paradise CR, Bradley EW, McGee-Lawrence ME, Im HJ, Karperien M, Krych AJ, Westendorf JJ, Larson AN, van Wijnen AJ.: Loss of histone methyltransferase Ezh2 stimulates an osteogenic transcriptional program in chondrocytes but does not affect cartilage development. J Biol Chem 2018:19001-19011.

15. van Oosterwijk JG, van Ruler MA, Hogendoorn PC, Dijkstra PD, van Rijswijk CS, Machado I, Llombart-Bosch A, Szuhai K, Bovée JV.: Three new chondrosarcoma cell lines: 
one grade III conventional central chondrosarcoma and two dedifferentiated chondrosarcomas of bone. BMC Cancer 2012:12:235.

16. Dudakovic A, Camilleri, ET, Xu, F., Riester, SM., McGee-Lawrence, ME., Bradley, EW., Paradise, CR., Lewallen, EA., Thaler R., Deyle DR, Larson AN., Lewallen DG., Dietz AB., Stein GS., Montecino MA., Westendorf JJ., and van Wijnen AJ.: Epigenetic control of skeletal development by the histone methyltransferase Ezh2. J BiolChem 2015:2760427617.

17. Fagerberg L, Oksvold P, Kampf C, Djureinovic D, Odeberg J, Habuka M, Tahmasebpoor S, Danielsson A, Edlund K, Asplund A, Sjöstedt E, Lundberg E, Szigyarto CA, Skogs M, Takanen JO, Berling H, Tegel H, Mulder J, Nilsson P, Schwenk JM, Lindskog: Analysis of the human tissue-specific expression by genome-wide integration of transcriptomics and antibody-based proteomics. Mol Cell Proteomics 2014:397-406.

18. Nowick K, Stubbs L. Lineage-specific transcription factors and the evolution of gene regulatory networks. Brief Funct Genomics. 2010 Jan; 9(1): 65-78.

19. Vaquerizas JM, Kummerfeld SK, Tecihmann SA, Luscombe NM. A census of human transcription factors: function, expression and evolution. Nature Reviews Genetics (2009) volume10, pages252-263.

20. Vissing H, Meyer WK, Aagaard L, Tommerup N, Thiesen HJ. Repression of transcriptional activity by heterologous KRAB domains present in zinc finger proteins. FEBS Lett. 1995 Aug 7;369(2-3):153-7.

21. Fedotova AA, Bonchuk AN, Mogila VA, Georgiev PG. C2H2 Zinc Finger Proteins: The Largest but Poorly Explored Family of Higher Eukaryotic Transcription Factors. Acta Naturae. 2017 Apr-Jun; 9(2):47-58.

22. Wingender, E., Schoeps, T. and Dönitz, J.: TFClass: An expandable hierarchical classification of human transcription factors. Nucleic Acids Res. 41, D165-D170 (2013). doi: 10.1093/nar/gks1123.

23. Lachmann A, Torre D, Keenan AB, Jagodnik KM, Lee HJ, Wang L, Silverstein MC, Ma'ayan A. Massive mining of publicly available RNA-seq data from human and mouse. Nature Communications 9. Article number: 1366 (2018), doi:10.1038/s41467-01803751-6.

24. Sandelin, A. JASPAR: an open-access database for eukaryotic transcription factor binding profiles. Nucleic Acids Res. 32:D91-4.

25. Mathelier, A. JASPAR 2014: an extensively expanded and updated open-access database of transcription factor binding profiles. Nucleic Acids Res. 42:D142-7.

26. Friedman, RC. Most mammalian mRNAs are conserved targets of microRNAs. Genome Res. 19:92-105.

27. Grimson, A. MicroRNA targeting specificity in mammals: determinants beyond seed pairing. Mol Cell. 27:91-105.

28. Garcia, DM. Weak seed-pairing stability and high target-site abundance decrease the proficiency of lsy-6 and other microRNAs. Nat Struct Mol Biol. 18:1139-46.

29. Hecht J, Stricker S, Wiecha U, Stiege A, Panapoulou G, Podsiadlowski L, Poustka AJ, Dieterich C, Ehrich L, Suvorova J, Mundlos S, Seitz V. Evolution of a Core Gene Network for Skeletogenesis in Chordates. PLoS Genet. 2008 Mar; 4(3): e1000025. doi: 10.1371/journal.pgen.1000025

30. Takigawa Y, Hata K, Muramatsu S, Amano K, Ono K, Wakabayashi M, Matsuda A, Takada K, Nishimura R, Yoneda T. The transcription factor Znf219 regulates chondrocyte 
differentiation by assembling a transcription factory with Sox9. J Cell Sci. 2010 Nov 1;123(Pt 21):3780-8. doi: 10.1242/jcs.071373. Epub 2010 Oct 12.

31. Okada K, Fukai A, Mori D, Hosaka Y, Yano F, Chung UI, Kawaguchi H, Tanaka S, Ikeda T, Saito T. Identification of SCAN Domain Zinc-Finger Gene ZNF449 as a Novel Factor of Chondrogenesis. PLoS One. 2014; 9(12): e115169. Published online 2014 Dec 29. doi: 10.1371/journal.pone.0115169 


\section{CHAPTER 8}

\section{Summary, General Discussion and Outlook}




\subsection{Mesenchymal Stem Cells versus Medicinal Signaling Cells}

Over the past decade, tissue engineering has become the prospective solution from defects resulting from severe trauma, osteodegenerative diseases and mechanical stress [1]. Tissue engineering requires the use of different cell types, scaffolds, growth factors and genetic stimuli to repair or reconstitute articular cartilage [2]. However, choosing an adequate cell type for these strategies is crucial. Two different notions about the use of MSCs have been described. The first notion proposes the term of medicinal signaling cells (MSCs) and relies in the trophic functions of MSCs where these cells survey the damage, isolate foreign components, stabilize the injured tissues, provide antibiotics, antiinflammatory components and pro-regenerative components and encysting protection before a medicinal sequence can be initiated to regenerate the damaged tissue $[3,4]$. In the second notion, the multilineage capacity and differentiation potential of MSCs is exploited to provide new chondrocytes.

Sixteen years after stem cells were first discovered, Urist et al. introduced the term 'mesenchymal stem cells' to highlight the multilineage differentiation potential of these cells [5-6]. Collectively, his work showed that these cells could differentiate into cartilage, bone and fat. Since then, several cell therapies, MSC based products and different treatments have been studied. Around 3,000 research articles referring to MSCs have been published every year. However, since 2010, Caplan retracted from his initial label of calling MSCs stem cells and proposed a new name: Medicinal Signaling Cell. This name change was based on the ability of these cells to secrete proteins that modulate the immune response, reduce inflammation, promote wound healing and inhibit cell death [4]. Previous data confirmed that the implantation of MSCs in vivo into cartilage defects, induced desirable healing outcomes in treated patients [7]. Riester et al. showed that injection of aMSCs in early stages of OA inhibited synovial thickening and cartilage loss [8]. 
Although, the notion of these cells to differentiate in vitro is still valid, their heterogeneous behavior represents a negative factor for the in vivo treatments [1]. In this thesis we used the potential of MSCs to differentiate into chondrocytes and presented different molecular mechanisms to enhance their in vitro differentiation potential. The overall goal was to pre-program the cells before implantation to improve in vivo results. However, or biggest limitation lies in that no in vivo validation of these strategies was performed.

\subsection{Hypoxia improves in vitro chondrogenic differentiation of MSCs.}

The mechanism by which articular cartilage homeostasis is maintained and is able to resist hypertrophic differentiation and subsequent endochondral ossification remains unknown. Thus, the complex molecular events occurring in the induction and maintenance of the chondrogenic phenotype during embryogenesis may lead to the identification of novel mechanisms involved in cartilage formation. The importance of the hypoxic environment for cartilage tissue engineering was highlighted in Chapter 2. Pre-programming the cells in the cartilage native physiological conditions may be helpful for enhancing the chondrogenic phenotype while preparing the cells for implantation.

In Chapter 3, the chondrogenic differentiation potential of aMSC expanded in human platelet lysate was examined. This study differed from previous aMSCs studies because of the use of a non-xenogeneic culture serum, which is applicable for clinical translation. Our findings indicated that genetic programming of aMSCs to a chondrocytic phenotype is effective in cellular aggregates (pellet culture) under hypoxic conditions as reflected by increased expression of cartilage-related biomarkers and biosynthesis of a glycosaminoglycan positive matrix. These results were also found by Leijten and colleagues during chondrogenic differentiation of bone marrow derived bMSCs [9]. Formation of cartilaginous tissues with sufficient collagen type II and proteoglycans is essential to reconstruct the cartilage with restored functions. The oxygen status 
was a dominant environmental parameter for directing chondrogenic differentiation of aMSCs and reduced their tendency to generate a fibroblastic ECM, while helping chondrocytes to maintain their phenotype by enhancing. Hence, hypoxia was more effective in promoting a chondrocytic phenotype at earlier stages of differentiation.

Furthermore, Chapters 4 and $\mathbf{5}$ studied the importance of signaling molecules such as miRNAs for cartilage development, maintenance, and destruction [1013]. We proposed that hypoxia stimulated chondrogenesis of MSCs is mainly driven by upregulation of miR-210. Functional experiments revealed miR-210 importance for early chondrogenesis of MSCs during in vitro cultures. Exogenous miR-210 expression can be used instead of chondrogenic differentiation cocktail in standard or low oxygen cultures to promote chondrogenesis of MSCs. Remarkably the use of miR-210 mimic does not present negative side effects such as increased in COL1A1 expression or induction of hypertrophic markers compared to TGF $\beta 1$. This potential treatment can help regenerating hyaline cartilage whilst preventing formation of fibrocartilage in vivo. Although positive results were achieved from previous experiments, there still a need to validate that this degree of differentiation achieved in vitro is stable for implantation, and will maintain the phenotype and functionality of the cells over time.

\subsection{Synovial fluid is an alternative culture serum for in vitro expansion of MSCs and chondrocytes for clinical therapies}

Fetal bovine serum (FBS) has long been the gold standard medium supplement for laboratory-scale MSC culture and other cells. However, FBS has a poorly characterized composition and poses risk factors, as it may be a source of xenogenic antigens and zoonotic infections [14]. The need for an alternative is known to scientists and users involved in cell therapy or advanced therapy medicinal products. 
Since concerns have been raised regarding the safety of FBS other serums have been proven with some success [15]. As an alternative, platelet lysate (PL) can be an adequate non-xenogenic alternative for fetal calf serum. However, PL triggers an inflammatory response in MSCs and induces the secretion of factors maintaining macrophages in a pro-inflammatory state [16]. In Chapter 6, SF was used as an alternative for both FBS and PL. We examined if it could enhance, limit or reject stem cells or chondrocytes upon implantation. Our data indicated that synovial fluid enhances the metabolic activity of chondrocytes and aMSCs at a level that is at least equivalent to FBS and better than human alternative PL. Thus, we found synovial fluid to be a promising novel culture supplement for chondrocyte and aMSCs expansion and due to its non-zoonotic (animal free) nature is therefore potentially better for clinical applications in cartilage regeneration. However, the limitations of synovial fluid were highlighted in the chapter. The differences found between the SF samples during cell culture, suggested further normalization of the samples would be essential to preserve both stem cells and chondrocytes viability and maintenance. Because concentrations of synovial fluid protein are known to vary with the degree of joint inflammation, comparing the protein profile to determine potential key components that could influence cell culture is important for the normalization of the synovial fluid. Thus, correlation between viability, protein content and cytokines profile could help for the stratification of samples for future therapeutic use.

\subsection{Cartilage specific transcription factors could improve cartilage regeneration and prevent osteoarthritis development.}

The complex molecular events occurring in the induction and maintenance of the chondrogenic phenotype during embryogenesis may lead to the identification of novel mechanisms involved in cartilage formation. Fundamental knowledge of 
these mechanisms might prove transcription factors such as Zinc Finger 648 (ZNF648) protein to be crucial for cartilage repair strategies (Chapter 7).

ZNF648 that belongs to the transcription factor family C2H2-ZNF is part of a large family of TFs, which have tissue-specific expression and are tought to control processes that are characteristic of individual tissues. However, the biological functions of most of these TFs are unknown [19]. In our study, cartilage-specific transcription factor ZNF648 protein seems to maintain normal differentiation of pre-committed chondrogenic mesenchymal progenitor cells into chondrocytes in the growth plate, whereas in later stages of cartilage maturation ZNF648 expression was reduced.

Further study will help elucidate ZNF648 signaling mechanisms for cartilage development. Previously a list of potential target genes of ZNF648 exhibited several cartilage related genes including SOX2, SOX5, HAPLN1, GLI1, SOX9, RUNX2, while targeting hypoxia related genes ARNT (HIF1B), HIF1A [22-25]. The differential gene expression observed in our study between growth plate and articular chondrocytes, could indicate a regulatory mechanism of ZNF648 to promote chondrogenesis and cartilage formation; this could occur by regulation of other cartilage specific genes and not necessarily of COL2A1, but by interacting with one or various pathways involved in cartilage development, including SOX9, RUNX2 and the hypoxic pathway.

\subsection{Concluding remarks and future perspectives.}

Tissue engineering offers possibilities for optimization of cartilage repair by combining different cell types, biomaterials, and growth factors for the support of cartilage regeneration [17]. Despite the efforts, current cell-based repair strategies have been proven unsuccessful for treating large cartilage defects and osteoarthritic lesions. Proper regeneration of this tissue remains an unresolved question and no standardized clinical protocol has yet been established. 
Engineered cartilage using MSCs may become hypertropic resulting in the production of bone-like or fibrotic tissues once implanted [18]. Several studies have attempted to understand the mechanisms by which MSCs can be programmed for chondrogenic differentiation while avoiding further progression into hypertrophic cartilage $[19,20]$. The physiological conditions in the articular cartilage are very complex consisting of low oxygen environment and repeated mechanical loading. These non-genetic strategies have become more atractive due to their easiness for manipulation. For in vitro cartilage engineering strategies, a delicate balance must be reached between the stimulatory and inhibitory effects of the low supply of oxygen. Although some biomaterials used in cartilage regeneration strategies mimic a hypoxic microenvironment for the cells, the cells are still subjected to normal oxygen levels in culture. However the equipment required to maintain the cells in a hypoxic microenvironment is often expensive and space restrictive. As a consequence, it would be interesting to perform in vitro chondrogenic differentiation of MSCs using a regulatory mechanism that would mimic the hypoxic response on the cells without the need of low oxygen cultures. This regulatory mechanism would also serve to avoid the dedifferentiation of the cells; enhance chondrogenic differentiation in vitro before implantation in the patient and /or to prevent the loss of chondrocyte phenotype during culture expansion.

In this thesis we studied different molecular mechanisms to potentiate chondrogenesis of MSCs during culture by achieving a similar gene expression and protein deposition to human primary chondrocytes. To validate the relevance of these molecular mechanism, their effects on human primary chondrocytes were also studied. Hypoxia, synovial fluid and transcription factor ZNF648 seemed relevant not only for chondrogenesis of MSCs but also to maintain the chondrocyte's phenotype. In Figure 8.1 a summary of the findings 
encountered during the development of this thesis will put in perspective the molecular mechanisms found and its interactions.

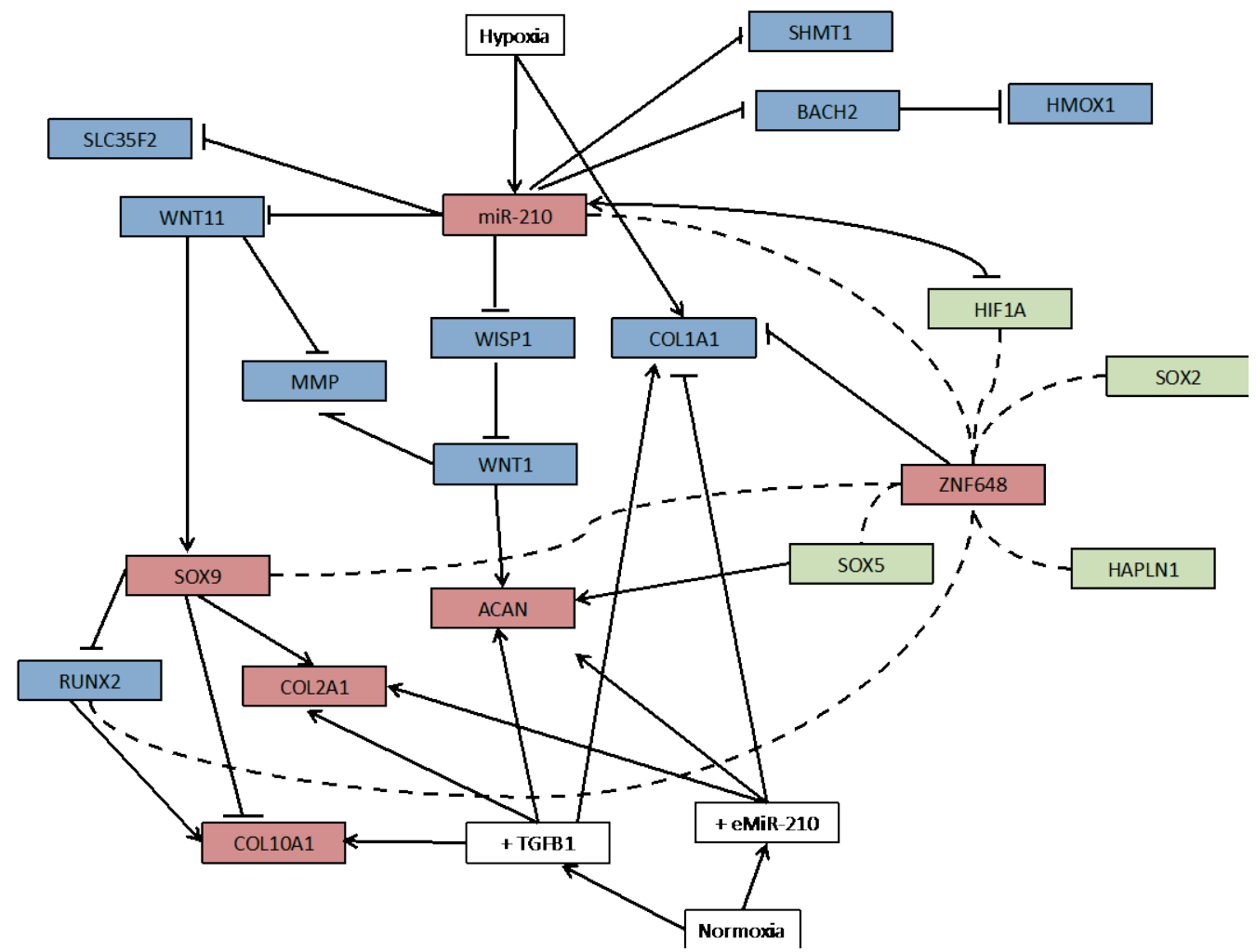

Figure 8.1 Molecular mechanisms modulating chondrogenesis. Up-regulated genes (red box), down-regulated genes (blue box) and inconclusive genes (green box). Dotted lines denoted molecular mechanisms suggested in the literature but not validated during the thesis development. For normoxia, addition of TGFB1 or exogenous miR-210 (eMiR-220) was denoted by a + symbol.

aMSCs differentiated under serum containing media with TGF $\beta 1$ and hypoxic conditions was found to increase the expression of COL2A1 and ACAN, but also increased the expression of hypertrophic markers. However, under hypoxic conditions, miR-210 was observed to be the main driver of chondrogenesis of stem cells, and proved that its induction enhances chondrogenic differentiation as strong as TGF $\beta 1$ addition in stem cells. Higher COL2A1/COL1A1 ratio on cells 
overexpressing miR-210 when compared to TGF $\beta 1$ induction, suggested miR210 could decreased the cells fibroblastic properties.

Altought miR-210 has been largely studied in relation to hypoxia, recently has been also linked to cartilage. Lin $\mathrm{L}$ et al. found miR-210 among the highly expressed miRNAs in human primary articular chondrocytes at early passages [22], whereas Zhang Z et al. and Georgi $\mathrm{N}$ et al. found miR-210 expressed during chondrogenic differentiation of aMSCs and bMSCs respectively [23, 24]. miR-210 has been related to RUNX2, SMAD 4, SMAS 5 and SOX2, which are all linked to chondrogenesis [27]. In vivo administration of miR-210 by intra-articular injection effectively promoted the healing of partly damaged anterior cruciate ligaments (ACLs) [28]. However, the efficacy to restore articular or growth plate cartilage in vivo is unknown. Microarray analysis found miR-210 down-regulated in OA cartilage compared with normal cartilage and suggested miR-210 as a negative regulator of the progression of $\mathrm{OA}$, which increases chondrocyte proliferation and prompts extracellular matrix deposition by directly targeting HIF-3 $\alpha[25,26]$. In our study, we found that exogenous addition of miR-210 to MScs in vitro promoted the expression of COL2A1 and ACAN while inhibiting the expression of COL1A1. However, we did not analyzed any of our cultures after 28 days, nor investigate its potential in vivo outcome.

Despite that miR-210 seems like a good candidate to promote chondrogenic differentiation, collagen type II and ACAN levels are still significantly lower than those from human primary chondrocytes. Consequently, the study of a transcription factor (ZNF648) that could regulate early development of chondrocytes would be essential for cartilage repair strategies. As observed in Chapter 7, ZNF648 may be an important regulatory mechanism during early development of the chondrocytes. It increased significantly the expression of collagen type II while decreasing expression of collagen type I in cells overexpressing ZNF648. Therefore, both ZNF648 and miR-210 are beneficial for 250 
earlier chondrogenesis of cells, suggesting a possible interaction between both factors. Both are significantly enhanced in cartilage native tissue, are expressed in low oxygen conditions and promote collagen type II and aggrecan expression. Target genes predicted miR-210 as one of the miRNAS involved with ZNF648 [26- 28]. Possibly, under low hypoxia, transcription factor ZNF648 could participate in promoting a cascade of genes that also regulate miR-210 expression (e.g. HIF1A). The expression of both factors at a certain time seems important during cartilage development and chondrogenesis. ZNF648 upregulation will induce genes and proteins important for cartilage maitenance (e.g. SOX9, COL2A1, ACAN) while miR-210 will create an equilibrium by inhibiting expression of cartilage degradation proteins (e.g. RUNX2, COL1A1, COL10A1, MMPs) (Figure 8.1.).

Altought SOX9 is vastly known as the master regulator of cartilage formation and chondrocyte differentiation, ZNF648 null mice indicated a possible role of ZNF648 during cartilage development and chondrocyte proliferation. Akiyama et al. revealed that SOX9 remains a central determinant for the maintenance of the lineage fate and multistep differentiation program of growth plate chondrocytes, whilst preventing osteoblastic differentiation and allowing hypertrophy during the prehypertrophy stage $[30,31]$. Our results showed than in immature cells, the decreased of ZNF648 was followed by down-regulation of SOX9. However, this was not observed for articular chondrocytes, SOX9 expression was preserved even when ZNF648 expression was lost. This suggested similar roles of ZNF648 and SOX9 during embryonic cartilage development, but a different role for cartilage homeostasis and maintenance. SOX9 is necessary both to specify and maintain the lineage choice of the cells and to activate stage-specific markers [32, 33]. We therefore propose, that SOX9 activates the expression of ZNF648 during cartilage development. Then, while SOX9 maitains its own expression through positive feedback during further cartilage development stages, once no longer 
needed, ZNF648 expression is inhibited by miR-210 mechanisms in a SOX9 dependent manner.

Taking into account the newer notion of the MSCs as trophic cells once implanted in vivo, it would be important to combine both the trophic functions and the differentiation potential to derive better MSCs for cell therapies. If these MSCs are better as signaling cells during injury and help to activate the necessary mechanisms of response during this stage, the co-cultures of these MSCs with primary chondrocytes would be an ideal candidate for cartilage repair. The MSCs would secrete the proteins that modulate the immune response, reduce inflammation, promote wound healing and inhibit cell death [21], while chondrocytes would proliferate and induce cartilage specific matrix production to repair the defect. Using the molecular mechanisms described in this thesis (Figure 8.1.) we could further enhance the differentiation potential of MSCs, which in combination with their own trophic effects could enhance therapeutic outcome. However, it is important to understand the interaction between these trophic functions, their differentiation potential and the efficacy after implantation. Lastly, to be used in cell therapies, several parameters need to be studied to optimize and augment therapeutic efficacy including optimal route of administration, the correct dose, the best cell populations and timing of cultures. 


\section{REFERENCES}

1. Pfander D, Cramer T, Schipani E, Johnson R: HIF-1alpha controls extracellular matrix synthesis by epiphyseal chondrocytes. J Cell Sci 2003, 116:1819-1826.

2. Silver I: Measurement of $\mathrm{pH}$ and ionic composition of pericellular sites. Philos Trans R Soc Lond B Biol Sci 1975, 271:261-272.

3. Caplan A: MSCs: The sentinel and safe guards of Injury. J Cell Physiol 2016, 7:1413-1416.

4. Sipp D, Robey PL: Clear up this stem cell mess. Nature 2018:455-457.

5. Urist M, Nogami H: Morphogenetic substratum for differentiation of cartilage in tissue culture. Nature 1970:1051-1052.

6. Caplan A, Syftestad G, Osdoby P: The development of embryonic bone and cartilage in tissue culture. Clin Orthop Relat Res 1983:243-263.

7. Treuhaft P, MCCarty D: Synovial fluid $\mathrm{pH}$, lactate, oxygen and carbon dioxide partial pressure in various joint diseases. Arthritis Rheum 1971, 14:475-484.

8. Riester SM, Jones D, Lin Y, Jones DL, de Mooij T, Lewallen EA, Nie H, Paradise CR, Radel DJ, Dudakovic A, Camilleri ET, Larson DR, Qu W, Krych AJ, Frick MA, Im HJ, Dietz AB, Smith J, van Wijnen AJ.: Safety Studies for Use of Adipose Tissue-Derived Mesenchymal Stromal/Stem Cells in a Rabbit Model for Osteoarthritis to Support a Phase I Clinical Trial. Stem Cells Transl Med 2017, 6:910-922.

9. Leijten J, Moreira Teixeira L, van Blitterswijk C, Post J, Karperien M: Metabolic programming of mesenchymal stromal cells by oxygen tension directs chondrogenic cell fate. Proc Natl Acad Sci USA 2014:13954-13959.

10. Sumiyoshi K, Ohgawara T.: Identification of miR-1 as a micro RNA that supports late-stage differentiation of growth cartilage cells Biochem Biophys Res Commun 2010:286-290.

11. Ukai T, Akutsu H, Umezawa A, Mochida J: MicroRNA-199a-3p, microRNA-193b, and microRNA-320c are correlated to aging and regulate human cartilage metabolism. J Orthop Res 2012:1915-1922.

12. Song J, Kim D, Han J, Chun CH, Jin EJ.: MicroRNA-181b regulates articular chondrocytes differentiation and cartilage integrity. Biochem Biophys Res Commun 2013:210-214.

13. Vonk LA, Dhert WJ, Saris DB, Creemers LB: Overexpression of hsa-miR-148a promotes cartilage production and inhibits cartilage degradation by osteoarthritic chondrocytes . Osteoarthritis Cartilage 2014:145-153.

14. Hemeda H, giebel B, Wagner W. Evaluation of human platelet lysate versus fetal bovine serum for culture of mesenchymal stromal cells. Cytotherapy 2014:170-180.

15. Heldin C, Betsholtz C, Johnsson M, Nister M, Ek L: Platelet-derived growth factor: mechanism of action and relation to oncogenes. J Cell Sci Suppl 1985:65-76.

16. Ulivi V, Tasso R, Cancedda R, Descalzi F: Mesenchymal Stem Cell Paracrine Activity Is Modulated by Platelet Lysate: Induction of an Inflammatory Response and Secretion of Factors Maintaining Macrophages in a Proinflammatory Phenotype. Stem Cells and Development 2014.

17. Heng B, Cao T, Lee E: Directing stem cell differentiation into the Chondrogenic lineage in vitro. Stem Cells 2004:1152-1167.

18. Yang J, Zhang Y, Yue K, Khademhosseini A: Cell laden hydrogels for osteochondral and cartilage tissue engineering. Acta Biomaterialia 2017:1-25. 
19. Ma B, Wu L, Kip M, van Blitterswijk C, Karperien M: Gene expression profiling of dedifferentiated human articular chondrocytes in monolayer culture. Osteoarthritis and Cartilage 2013:599-603.

20. Wu L, Georgi N, Post J, van Blitterswijk C, Karperien M: Trophic effects of mesenchymal stem cells increase chondrocyte proliferation and matrix formation. Tissue Eng Part A 2011:1425-1436.

21. Bagno L, Hatzistergos K, Balkan W, Hare J: Mesenchymal Stem Cell-Based Therapy for Cardiovascular Disease: Progress and Challenges. Molecular Therapy 2018, 26:1610-1623.

22. Lin, L, Shen, Q, Zhang, C, Chen, L, Yu, C. Assessment of the profiling microRNA expression of differentiated and dedifferentiated human adult articular chondrocytes.J Orthop Res 2011; 29: 1578- 84.

23. Zhang Z, Kang Y, Zhang H, et al. Expression of microRNAs during chondrogenesis of human adipose-derived stem cells. Osteoarthritis Cartilage. 2012; 20: 1638-46.

24. Georgi N, Taipaleenmaki H, Raiss CC, Groen N, Portalska KJ, van Blitterswijk C, de Boer J, Post JN, van Wijnen AJ, Karperien M: MicroRNA Levels as Prognostic Markers for the Differentiation Potential of Human Mesenchymal Stromal Cell Donors. Stem Cells Dev 2015, 24:1946-1955.

25. Iliopoulos $\mathrm{D}$, Malizos $\mathrm{KN}$, Oikonomou $\mathrm{P}$, et al. Integrative microRNA and proteomic approaches identify novel osteoarthritis genes and their collaborative metabolic and inflammatory networks. PLoS ONE. 2008; 3: e3740.

26. Li Z, Meng D, Li G, Xu J, Tian K, Li Y. Overexpression of microRNA-210 promotes chondrocyte proliferation and extracellular matrix deposition by targeting HIF-3 $\alpha$ in osteoarthritis. Molecular medicine reports. 2016 Feb. Pages: 2769-2776. https://doi.org/10.3892/mmr.2016.4878.

27. Zhang Z, Liao W, Zhang Z, Duan X, Hou C. Expression of microRNAs during chondrogenesis of human adipose-derived stem cells. Osteoarthritis and cartilage. 2013 Apr. 21: S214-S215.

28. Shoji T, Nakasa T, Yamasaki $K$, et al. The effect of intra-articular injection of microRNA-210 on ligament healing in a rat model. Am J Sports Med. 2012; 40: 24708.

29. Akiyama, H. (2008). Control of chondrogenesis by the transcription factor Sox9. Mod. Rheumatol. 18, 213-219.

30. Akiyama, H., Chaboissier, M.C., Martin, J.F., Schedl, A., and de Crombrugghe, B. (2002). The transcription factor Sox9 has essential roles in successive steps of the chondrocyte differentiation pathway and is required for expression of Sox5 and Sox6. Genes Dev. 16, 2813-2828.

32. Kumar, D, Lassar, AB. The transcriptional activity of Sox 9 in chondrocytes is regulated by RhoA signaling and actin polymerization. Mol. Cell. Biol. 2009. 29, 42624273.

33. Leung VYL, Gao B, Leung KKH, Melhado IG, Wynn SL, Au TYK, et al. (2011) SOX9 Governs Differentiation Stage-Specific Gene Expression in Growth Plate Chondrocytes via Direct Concomitant Transactivation and Repression. PLoS Genet 7(11): e1002356. https://doi.org/10.1371/journal.pgen.1002356 


\section{Curriculum Vitae}

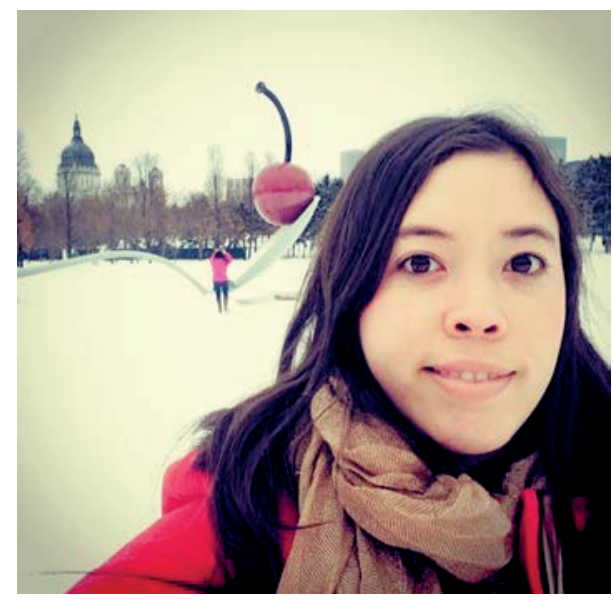

Catalina Galeano Garces was born on the $11^{\text {th }}$ of June 1990 in Medellin, Colombia. In 2008 she started her studies in Biomedical Engineering at EIA University-CES University in Envigado, Colombia. During her studies she participated in several traineeships in biotechnology and biomechanics. In December 2013 she received her bachelors diploma with an undergradute honorable mention. During her bachelor's she moved to France to pursue a master degree in engineering at Ecole Superieur des Arts et Metiers (Arts et Metiers ParisTech). During her master's she participated as a summer intern at the VU medical center in Amsterdam, The Netherlands under the supervision of Dr. Marco Helder in a project titled "Cell-matrix interactions in pelvic organ prolapse". Then she continued with a Master of Sciences in Tissue Engineering and Tissue Biomechanics from the Paris Institute of Technology (ParisTech). In April 2014 she graduated and obtained her MEng and MSc diplomas. Her master Project was focused on Cell adhesion control on implantable biomedical devices and was performed under the supervision of Dr. Vincent Semetey at the Institut Curie in Paris. Since 2013 she has been working under the supervision of Dr. Andre van Wijnen at the Department of Orthopedic Surgery at Mayo Clinic. In 2015, she started her $\mathrm{PhD}$ at the department of Developmental BioEngineering, The Netherlands, under the supervision of Prof. Dr. Marcel Karperien and Prof. Dr. Andre van Wijnen. The subject of her research was the identification of molecular mechanisms that could improve chondrogenesis of stem cells for cartilage regeneration therapies. 


\section{List of Publications}

\section{Peer Reviewed Papers}

Camilleri ET, Gustafson MP, Dudakovic A, Riester SM, Galeano-Garces C, Paradise CR, Takai H, Karperien M, Cool S, Sampen H-J, Larson AN, Qu W, Smith J, Dietz $\mathrm{AB}$, van Wijnen AJ. Identification and validation of multiple cell surface markers of clinical-grade adipose-derived mesenchymal stromal cells as novel release criteria for good manufacturing practice-compliant production. Stem Cell Res Ther. 2016 Aug 11;7(1):107. doi: 10.1186/s13287-016-0370-8.

Lin Y, Lewallen EA, Camilleri ET, Bonin CA, Jones DL, Dudakovic A, GaleanoGarces C, Wang W, Karperien MJ, Larson AN, Dahm DL, Stuart MJ, Levy BA, Smith J, Ryssman DB, Westendorf JJ, Im HJ, van Wijnen AJ, Riester SM, Krych AJ. RNA-seq analysis of osteochondral allografts reveals activation of early response genes and reduced expression of extracellular matrix-related genes. J Orthop Res. 2016 Nov;34(11):1950-1959. doi: 10.1002/jor.23209. Epub 2016 Mar 3.

Galeano-Garces C, Camilleri ET, Riester SM, Dudakovic A, Larson DR, Qu W, Smith J, Dietz AB, Im HJ, Krych AJ, Larson AN, Karperien M, van Wijnen AJ. Molecular validation of chondrogenic differentiation and hypoxia responsiveness of platelet-lysate expanded adipose-tissue derived human mesenchymal stromal cells. Cartilage. a2017 Jul;8(3):283-299. doi: 10.1177/1947603516659344. Epub 2016 Jul 21. (Chapter 3).

Paradise CR, Galeano-Garces C, Galeano-Garces D, Dudakovic A, Milbrandt TA, Saris DBF, Krych AJ, Karperien M, Ferguson GB, Evseenko D, Riester SM, van Wijnen AJ, Larson AN. Molecular characterization of physis tissue by next generation RNA sequencing. Gene. 2018 Aug 20;668:87-96. doi: 10.1016/j.gene.2018.05.034. Epub 2018 May 25.

Camilleri E, Dudakovic A, Riester SM, Galeano-Garces C, Paradise CR, Bradley EW, McGee-Lawrence ME, Im HJ, Karperien M, Krych AJ, Westendorf JJ, Larson $\mathrm{AN}$, van Wijnen AJ. Loss of histone methyltransferase EZH2 stimulates an osteogenic transcriptional program in chondrocytes but does not affect cartilage development. J Biol Chem. 2018 Oct 16. pii: jbc.RA118.003909. doi: 10.1074/jbc.RA118.003909. 
Hevesi M, Paradise C, Paggi C, Galeano-Garces C, Dudakovic A, Karperien M, Kakar S, Hewett T, Krych A, van Wijnen AJ. Defining the baseline transcriptional fingerprint of Rabbit Hamstring Autograft. Gene Reports. 2019 June. (15). doi.org/10.1016/j.genrep.2019.100363

Hevesi, M. Denbeigh J, Paggi C, Galeano-Garces C, Bagueri L, Stuart M, Saris, D, van Wijnen A, Krych A. Fresh osteochondral allograft transplantation in the knee: optimizing graft viability and expanding resources using a Living Donor Cartilage Program. Submitted.

Galeano-Garces C, Galeano-Garces D, Larson AN, van Wijnen A, Karperien M. Hypoxia regulatory mechanisms during cartilage development and cartilage-tissue engineering. (Chapter 2). In preparation.

Galeano-Garces C, Galeano-Garces D, Larson AN, van Wijnen A, Karperien M. Integrated analysis of miRNA-seq and mRNA-seq on hypoxic mesenchymal stem cells reveals the role of hypoxia-regulated miRNAs for promoting chondrogenesis of MSCs. (Chapter 4). In preparation.

Galeano-Garces C, Galeano-Garces D, Paggi C, Camilleri ET, Larson AN, van Wijnen A, Karperien M. miRNA-210 promotes chondrogenesis of MSCs. (Chapter 5). In preparation.

Galeano-Garces C, Galeano-Garces D, Ryan H, Qu W, Smith J, Camilleri ET, Riester S, Dudakovic A, Larson AN, Karperien M, van Wijnen AJ. Human synovial fluid assists human primary chondrocytes and mesenchymal stem cells chondrogenic differentiation during culture expansion. (Chapter 6). In preparation.

Galeano-Garces C, Galeano-Garces D, Thaler R, Pichurin O, Bouska G, Milbrandt T, Karperien M, van Wijnen AJ, Larson AN. Novel transcription factor ZNF648 might regulate cartilage development and homeostasis through the modulation of cartilage related signaling pathways. (Chapter 7). In preparation.

\section{Abstracts selected for poster presentation}

Galeano-Garces C, Camilleri ET, Riester S, Dudakovic A, Kakar S, Westendorf JJ, Smith J, Dietz AB, Cool SM, Im HJ, Krych AJ, Karperien M, and van Wijnen AJ. Molecular validation of chondrogenic differentiation and hypoxia responsiveness of adipose-tissue derived human mesenchymal stem cells. International Cartilage Repair Society (ICRS) World Congress 2015, Chicago USA.

Galeano-Garces C, Camilleri ET, Riester S, Dudakovic A, Wenchun Q, Smith J, Dietz AB, Im HJ, Krych AJ, Karperien M, and van Wijnen AJ. Chondrogenic 
differentiation of platelet-lysate expanded clinical grade adipose-tissue derived human mesenchymal stem cells in hypoxia. Orthopedic Research Society (ORS) Meeting 2016. Orlando, USA.

Galeano-Garces C, Camilleri ET, Riester S, Smith J, Dietz AB, Karperien M, and van Wijnen AJ. Effect of human synovial fluid in models of in vitro chondrogenic differentiation on Mesenchymal stem cells and human primary chondrocytes. Osteoarthritis Research Society (OARSI) Meeting 2016. Amsterdam, The Netherlands. Published on Osteoarthritis and Cartilage Journal, April 2016, Vol 24 , Supplemental 1 , Page S166 doi: http://dx.doi.org/10.1016/j.joca.2016.01.326).

Galeano-Garces C, Milbrandt T, Riester SM, van Wijnen AJ, Camillery ET, Zhang C, Liu H, Puleo D, and Larson AN. Restoring the Damaged Growth Plate Using a Drug and Cell Loaded PGLA Scaffold in a Rabbit Model. ORS and AAOS The Physis: Fundamental Knowledge To A Fantastic Future Through Research, February 2018, Rosemont, USA. 


\section{Acknowledgment}

The $\mathrm{PhD}$ is the biggest goal that I have accomplished so far, and I hope it will bring for me great new challenges in life.

First, I would like to thank God, he has gotten me through this process and has helped me stayed focused and strong throughout the years. For hearing my many prayers for the different projects and for putting the right people at the right time close to me.

I would like to thank both my advisors, Andre van Wijnen, and Marcel Karperien. Without their support, guidance, and opportunities this life changing experience would have never been possible. I am forever grateful to Andre for taking me into his lab. For the advice, and teachings that I received during these 6 years. I am sure they helped me grow into a better scientist. Marcel for opening this door of the $\mathrm{PhD}$, for the many meetings late at night and for helping me put everything together into my thesis dissertation.

For their great comments, and suggestions I want to say thank you to my committee members, the time you spent, and your thoroughness are highly appreciated and have helped me significantly improve my PhD thesis, and my writing/editing skills.

Emily, Scott and Amel, without you guys (the starters!) this would have been even crazier. You were such great mentors, and you were able to guide me and transfer so much knowledge to me selfishly. I have become a much better scientist thanks to you guys.

To my lab members thanks for these amazing years, your support, your ideas, the extra time in and outside of the lab and the memories that will stay forever. Specially to Daniela, Eva and Liz for helping me at the end of the road when things got intense. For sharing their patience, advice, love and mouse skills. For letting me overstay at their homes when I moved to Minneapolis (I bet Daniel strongly agrees with me), for feeding me and for staying until 4 am while we tried to figure out what was happening in our different projects (or why they weren't working) and lifes. I love you girls.

Negro, Manitas, y Ardilla. A mis 3 casi roomates. Por escucharme, por dejarme llorar, por reganarme cuando pasaba horas infinitas en el laboratorio y por hacerme entender que hay cosas mas importantes que el trabajo. Rochester no hubiera sido lo que fue sin ustedes. Gracias por su apoyo, tiempo, consejos y 
sabiduría. Por compartir con nosotros todos los momentos (hasta los feos) y por ayudar (cada uno por su parte) a que Daniel y yo estemos juntos hoy.

A a mis padres (mis chiplos) por darme todo el apoyo desde siempre, desde que este doctorado era solo un sueno. Por hacerme creer en mi en los momentos que me quería rendir. Por ensenarme a orar y a fortalecer esa $\mathrm{Fe}$ que mueve montanas. Por venir a aguantar frio con nosotros y hasta acompañarme a trabajar los fines de semana. Por amarme incondicionalmente, y por regalarme tantos momentos de felicidad. Los amo infinitamente.

A mi hermana, mi co-autora en casi todos los artículos. Por ese ano y medio que fuiste el otro cerebro y las otras dos manos de este trabajo. Este PhD es $2 / 3$ mio y $1 / 3$ tuyo. Por ayudarme en todos los experimentos, quedarte conmigo sin dormir y por ser el bastoncito que fuiste durante ese tiempo. Gracias a ti aprendi a no tener miedo de equivocarme y a creer mas en mi. Te amo mi peto.

Finalmente a mi esposo, por ayudarme tantas veces en el diseño y ejecución de presentaciones, artículos y hasta hacerme la portada de esta tesis. Por haber sido mi roca desde que llegaste a Rochester. Por apoyarme, escucharme, entenderme, trasnochar conmigo y alimentarme a altas horas de la noche mientras esperábamos un PCR o western blot. Por hacerme reir en los momentos dificiles. Por creer en mi, por no dejarme rendir, y por transmitirme mucha paz y paciencia. Je t'aime pour toujours. 